UC-NRLF

||| ||| || ||| || ||| || ||||||

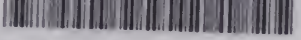

$\$ B>036 ?$ 


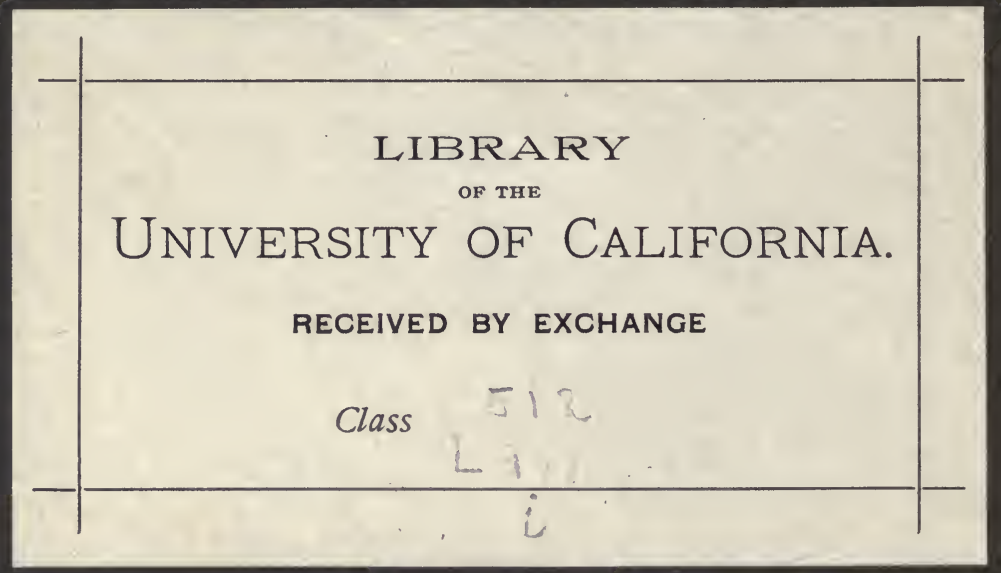




\section{IMPROVING THE QUALITY OF WHEAT}

BY

\section{T. L. LYON}

Thesis presented to the University Faculty of Cornell University for the Degree of Doctor of Philosophy 


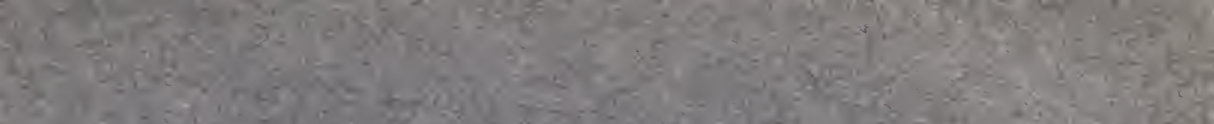

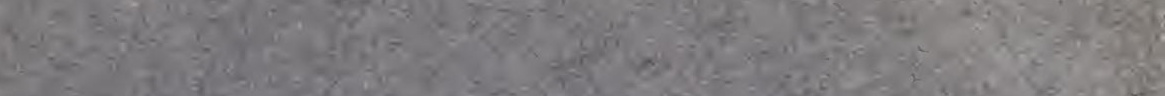

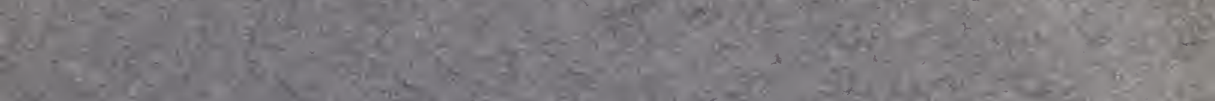

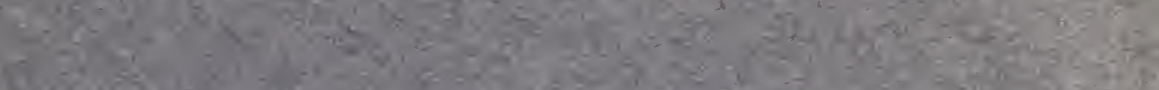

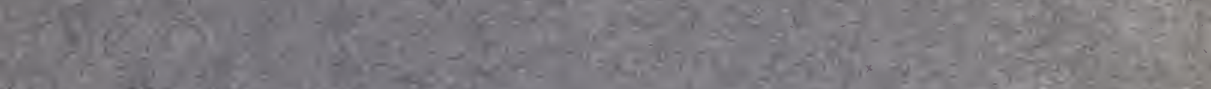

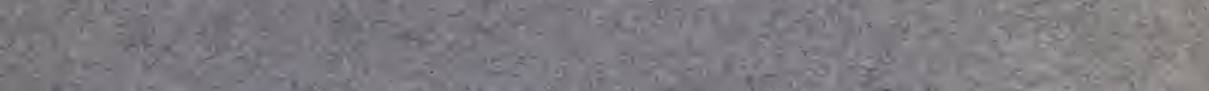

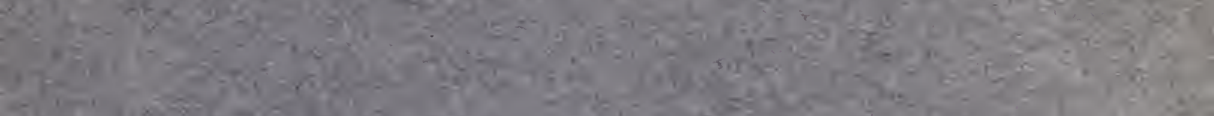

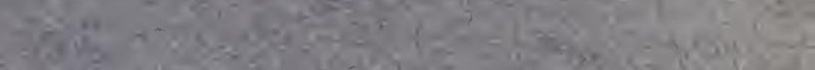

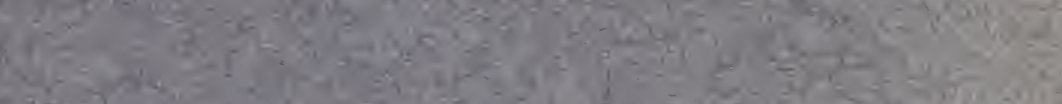

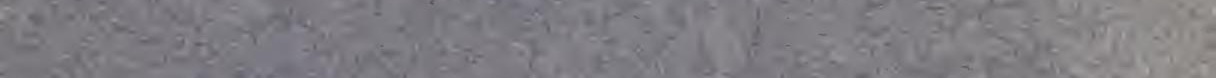

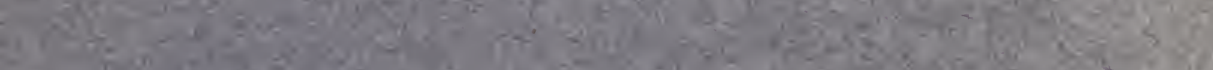

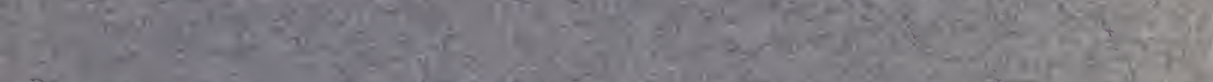

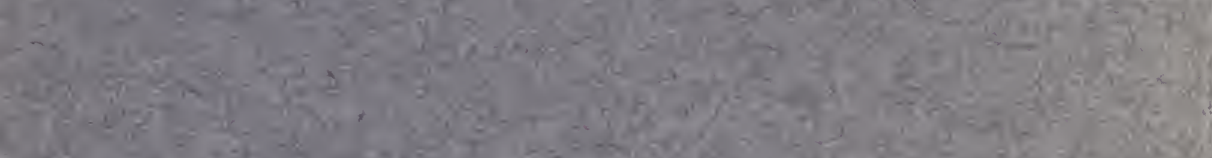

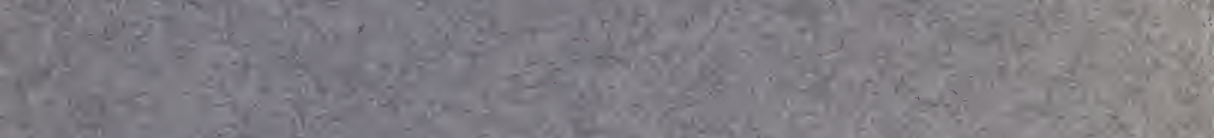

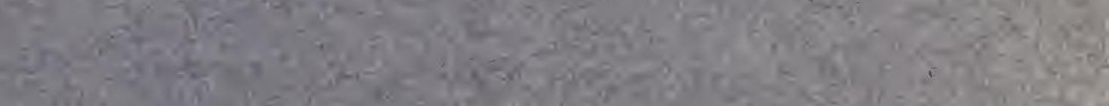

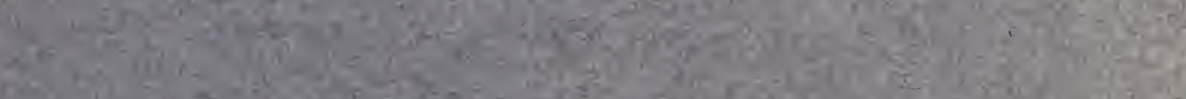
be

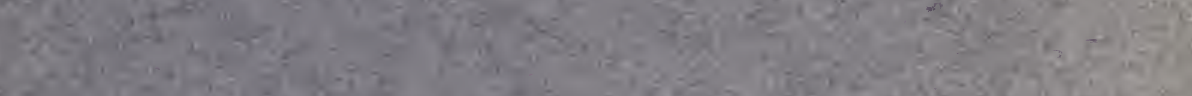

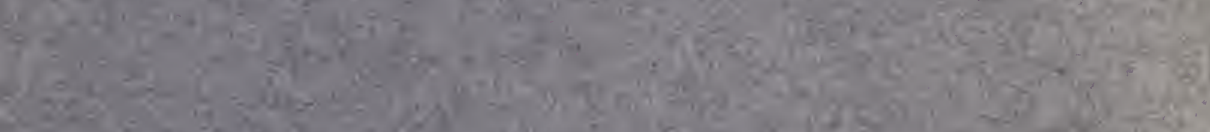

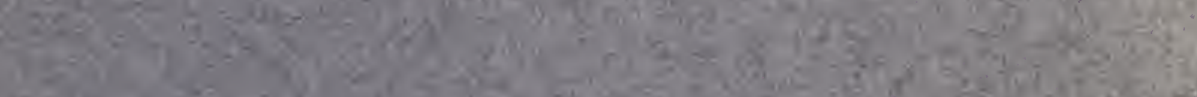

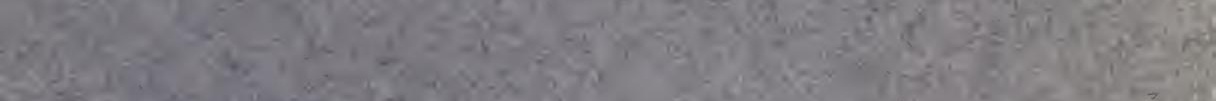

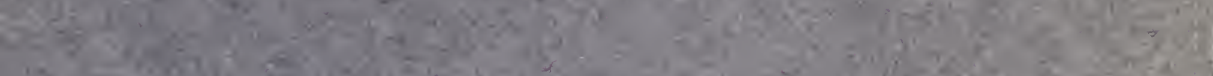
'P:

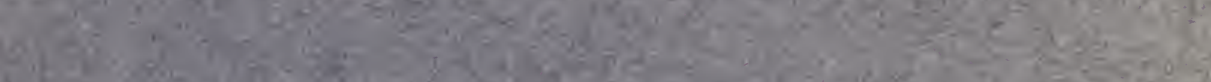
a

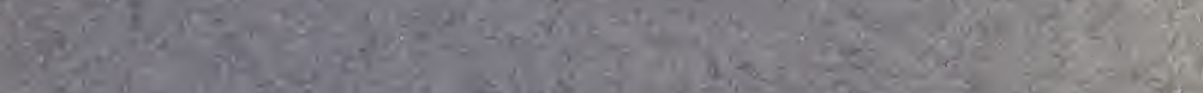

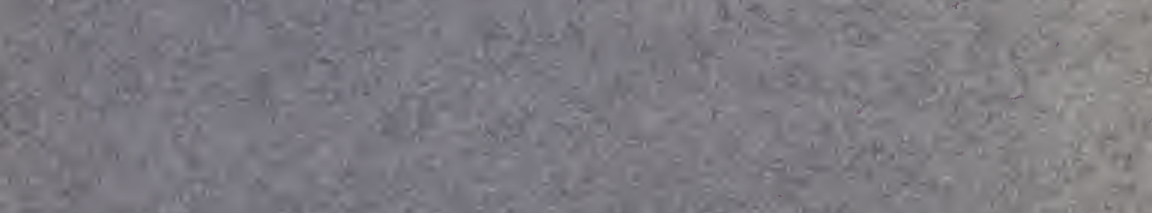
P.

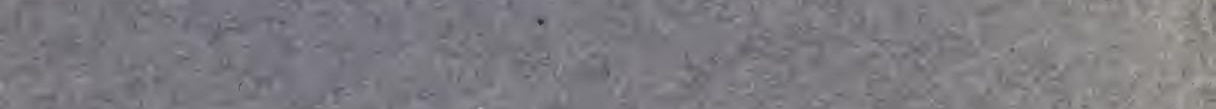

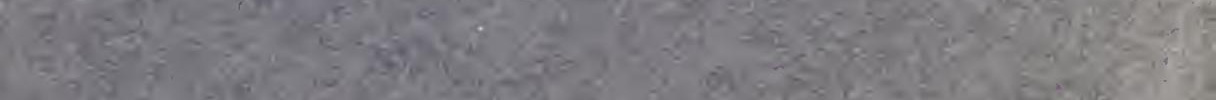

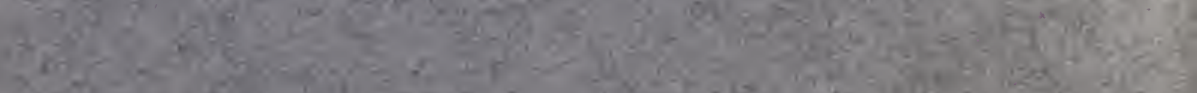

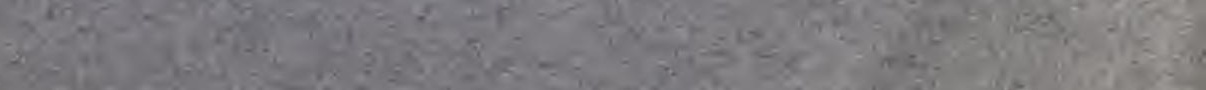




\section{IMPROVING THE QUALITY OF WHEAT}

BY

T. L. LYON

Thesis presented to the University Faculty of Cornell University for the Degree of Doctor of Philosophy

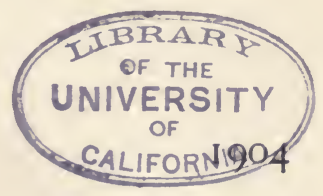

Also printed as Bulletin No. 78, Bureau of Plant Industry, U. S. Department of Agriculture 



\section{PREFACE.}

I wish to express my appreciation of the guidance of Professor I. P. Roberts, Professor G. C. Caldwell and Professor Thomas F. Hunt, who constituted the committee having my work in charge, also of the advice given by Dean L. H. Bailey and the assistance of Mr. G. N. Lauman. For the analytical work, extending through a period of seven years and involving several thousand chemical determinations, I am indebted to Professor S. Avery, Mr. R. S. Hiltner, Professor R. W. Thacher, Mr. Y. Nikaido, Miss Rachael Corr, Mr. H. B. Slade and Mr. G. H. Walker. Mr. Alvin Keyser has kept records of the wheat breeding plats and Mr. E. G. Montgomery has assisted in keeping other records. 



\section{CONTENTS.}

Object of the investigation

Part I.-Historical:

Some conditions affecting the composition and yield of wheat ........... 17

Composition as affected by time of cutting.................... 17

Influence of immature seed upon yield......................... 20

Influence of climate upon composition and yield.................. 20

Influence of soil upon composition and yield...................... 23

Influence of soil moisture upon composition and yield................ $\quad 29$

Influence of size or weight of the seed-wheat kernel upon the crop yield... 30

Relation of size of kernel to nitrogen content...................... . 35

Influence of the specific gravity of the seed kernel upon yield ......... 37

Relation of specific gravity of kernel to nitrogen content............. 39

Conditions affecting the production of nitrogen in the grain .......... 40

Part II.-Experimental:

Some properties of the wheat kernel........................... 49

Yield of nitrogen per acre.................................. $\quad 72$

Method for selection to increase the quantity of proteids in the kernel. ...... $\quad 76$

A basis for selection to increase the quantity of proteids in the endosperm of the kernel............................................ 84

Improrement in the quality of the gluten....................... 91

Some results of breeding to increase the content of proteid nitrogen. . . . . . 95

Yield of g. ain as affected by susceptibility to cold................... 100

Yield and nitrogen content of grain as affected by length of growing period.. 104

Relation of size of head to yield, height, and tillering of plant............ 111

Summary and conclusions.................................... 118 



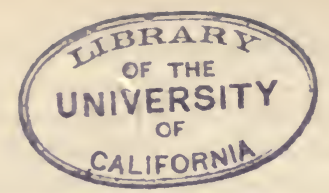

\section{TABLES OF EXPERIMENTS.}

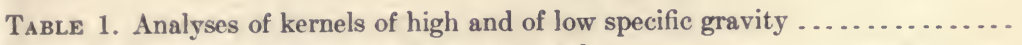

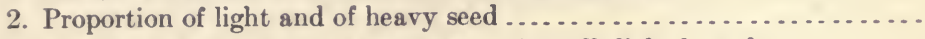

3. Analyses of large, heavy kernels and of small, light kernels..........

4. Analyses of spikes of wheat, arranged according to nitrogen content of

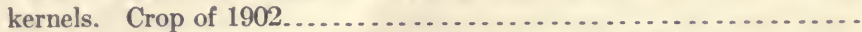

Page.

5. Summary of analyses of spikes of wheat, arranged according to nitrogen content of kernels. Crop of $1902 \ldots . . . \ldots \ldots . . . . . . . . . . .$.

6. Summary of analyses of spikes of wheat, arranged according to specific gravities of kernels. Crop of $1902 \ldots \ldots \ldots \ldots . . . . . . . . . . .$.

7. Summary of analyses of spikes of wheat, arranged according to weight of

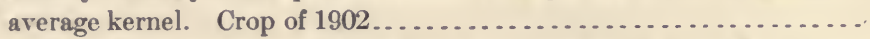

8. Analyses of plants, arranged according to percentage of proteid nitrogen.

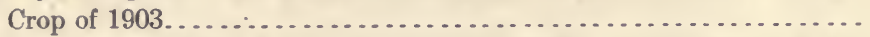

9. Summary of analyses of plants, arranged according to percentage of pro-

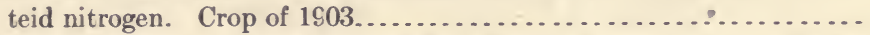

10. Analyses of plants, arranged according to weight of a verage kernel. Crop

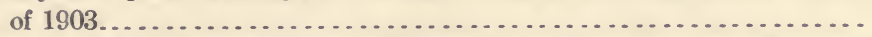

11. Summary of analyses of plants, arranged according to weight of average

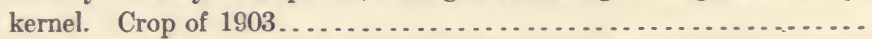

12. Summary of analyses of plants, arranged according to grams of proteid nitrogen in arerage kernel. Crop of $1903 . . . . . . . . . . . . . . .$.

13. Crops grown from light and from heavy seed for four years...........

14. Analyses of twenty-five spikes of wheat, showing their total organic nitro-

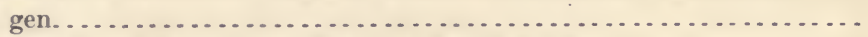

15. Analyses of twenty-three spikes of wheat, showing their percentage of

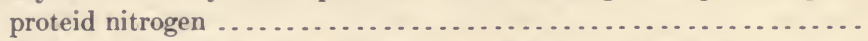

16. Analyses of twenty-one plants, showing total nitrogen and proteid nitrogen. . . . . . . . . . . . . . . . . . . . . . . . . . . . . .

17. Analyses of spikes of wheat, showing difference in proteid nitrogen ......

18. Variations in content of proteids............................

19. Relation of gliadin-plus-glutenin nitrogen to proteid nitrogen.........

20. Summary of analyses, showing relation of gliadin-plus-glutenin nitrogen

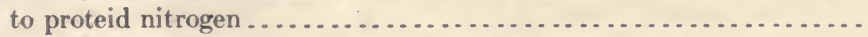

21. Relation of proteid nitrogen to gliadin-plus-glutenin nitrogen.........

22 . Summary of analyses, showing relation of proteid nitrogen to gliadin-plusglutenin nitrogen.................................

23. Ratio of gliadin to glutenin as the content of their sum increases......

24. Summary of analyses, showing the ratio of gliadin to glutenin as the content of their sum increases .............................

25. Analyses showing transmission of nitrogen from one generation to

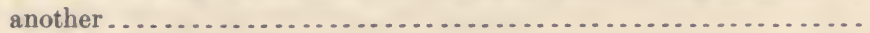


Page.

TABLE 26. Summary of analyses, showing transmission of nitrogen from one generation to another.

27. Analyses showing transmission of proteid nitrogen in average kernel... .

28. Analyses showing transmission of kernel weight ................... .

29. Yields of plants, arranged according to percentage killed in each family. .

30. Summary of yields of plants, arranged according to percentage killed in each family.

31. Yield and nitrogen content of grain, tabulated according to length of growing period

32. Summary of yield and nitrogen content of grain, tabulated according to length of growing period

33. Summary of nitrogen content, etc., tabulated according to yield per plant

34. Summary of yield, etc., tabulated according to nitrogen content........ .

35. Relation of size of head to yield, height, and tillering of plant......... . .

36. Summary of relation of size of head to yield, height, and tillering of plant .

37. Relation of yield of plant to height and tillering, and to the yield per head .

38. Relation of yield per head to yield, height, and tillering of plant, and to weight of average kernel. 


\section{IMPROVING THE QUALITY OF WHEAT.}

\section{OBJECT OF THE INVESTIGATION.}

Efforts to improve the wheat plant have been numerous and have accomplished important results. The work of Fultz, Clawson, Rudy, Wellman, Powers, Hayne, Bolton, Cobb, Green, and Hays in improving by selection, and of Pringle, Blount, Schindel, Saunders, Farrar, Jones, Carleton, and Hays in improving by hybridization, has resulted in giving this country many prolific strains and varieties of wheat, while Garton Brothers, of England, Farrar, of New South Wales, Vilmorin, of France, Rimpau, of Germany, and others have accomplished the same for other portions of the world. Attempts at improvement have, however, been directed primarily toward effecting an increase in the yield rather than in the quality of the crop. While the latter property has not been entirely lost sight of, selection based on quality has never been applied to the individual plant, but only to the progeny of otherwise desirable plants.

Why selection for quality of grain in the individual plant has not gone hand in hand with selection for other desirable properties is perhaps to be explained by the fact that no method for such selection has ever been devised. Mr. W. Farrar, of Queanbeyen, New South Wales, in an address made a short time ago, said:

Before we can make any considerable progress in improving the quality of the grain of the wheat plant we shall have to devise a method for making a fairly correct quantitative estimate of the constituents * * * of the grain of a single plant and yet have seeds left to propagate from that plant.

In devising a method for increasing the percentage of nitrogen in wheat it becomes desirable to know the causes that produce variation in this constituent of the kernel. Numerous experiments and observations have been made on this subject, the results of which agree in the main in attributing such variation to the following conditions:

(1) Stage of development of the kernel.

(2) Variation in temperature of different regions.

(3) Variation in temperature of different years in the same region.

(4) Variation in the supply and form of soil nitrogen.

(5) Variation in the supply of soil moisture. 
All of these factors have been studied, and are recognized as operative. Nothing, however, appears to have been done to show their influence upon the actual amount of nitrogen taken up by the wheat plant and deposited in the kernel. This is really the point of greatest interest; for although it is desirable to secure a wheat of greater nutritive value, it should not be done at the sacrifice of yield of nitrogenous substance.

Admitting that variation in the nitrogen content of wheat is induced by the conditions mentioned, it is essential to the plant breeder to know whether a high or low nitrogen content may be, under similar conditions, a characteristic of an individual plant; whether this quality is transmitted to the offspring; with what constant characteristics it is correlated, and whether a high percentage of nitrogen in a normal, perfectly matured wheat plant is an indication of a large accumulation of nitrogen by that plant.

The data contained in this paper cover the points mentioned, and it is hoped that some definite information has been gained that will lead to a practical solution of the problem of improving by breeding the quality of wheat for bread making. 
PARTI.

HISTORICAL. 



\section{SOME CONDITIONS AFFECTING THE COMPOSI- TION AND YIELD OF WHEAT.}

Experiments to ascertain the effect of different conditions upon the composition and yield of wheat have been conducted mainly along the following lines:

(1) Stage of growth at which the grain is harvested.

(2) Influence of immature seed upon the resulting crop.

(3) Effect of climate.

(4) Effect of soil.

(5) Effect of soil moisture.

(6) Influence of size or weight of seed upon the resulting crop.

(7) Influence of specific gravity of seed upon the resulting crop.

A brief summary of a number of these experiments is herewith given.

\section{COMPOSITION AS AFFECTED BY TIME OF CUTTING.}

In $1879,{ }^{a}$ and again in $1892,{ }^{b}$ Dr. R. C. Kedzie conducted very careful experiments to note the chemical changes that occur in the wheat kernel during its formation and ripening. These agree in the main in showing a gradual decrease in the percentage of total nitrogen, albuminoid nitrogen, and non-albuminoid nitrogen from the time the grain set to the time the kernel was ripe. The decrease in all of these constituents was much more rapid during the first than during the last stages of this development. The percentage of ash decreased at the same time.

In 1897 Prof. G. L. Teller ${ }^{c}$ carried on some experiments in which he covered the ground already gone over by Doctor Kedzie and also contributed to the knowledge of the subject some very important data concerning the proportion of the various proteids contained in the wheat kernel during the process of development. Teller found that the proportion of total nitrogen in the dry matter steadily decreased from the time the kernel was formed up to about a week before ripening, but that, unlike Doctor Kedzie's results, it gradually increased from that time on. He intimates that this increase before ripening may have been due to defective sampling and hoped to

a Report of Michigan Board of Agriculture, 1881-82, pp. 233-239.

$b$ Michigan Agricultural Experiment Station Bulletin 101.

c Arkansas Agricultural Experiment Station Bulletin 53. 
repeat the experiment to remedy this, but he has published nothing further. The amid nitrogen continued to decrease up to the time of ripening, as did also the ash, fats, fiber, dextrins, and pentosans. There was a gradual and marked increase in the proportion of gliadin up to the time of ripening, and a somewhat less and rather irregular decrease in the proportion of glutenin during the same period.

Failyer and Willard ${ }^{a}$ report analyses of wheat in the soft-dough stage and when ripe. The ash, crude fiber, fat, and the total and albuminoid nitrogen were higher in the soft-dough wheat, and the nitrogen-free extract and non-albuminoid nitrogen were higher in the ripe wheat.

Dietrich and König ${ }^{b}$ quote results from five experimenters-Reiset, Stockhardt, Heinrich, Nowacki, and Handtke. Only in one case (Heinrich) is there a constant decrease in total nitrogen as the grain approaches ripeness. There is much inconstancy in the results, there being in some cases a decrease in nitrogen between the milk stage and full ripeness and sometimes an increase. There is little information to be gained from the results quoted by Dietrich and König.

Körnicke and Werner in their "Handbuch des Getreidebaues" refer to the work of Stockhardt, and also that of Heinrich, to show that during the process of ripening the percentage of nitrogen in the wheat kernel gradually diminishes, as does also the percentage of ash, and that, on the other hand, the percentage of carbohydrates increases during the same period. Heinrich also shows by a statement of the number of grams of these constituents in 2,600 kernels at different stages of development that the absolute amount of nitrogen and ash increases up to the time of ripening, and that consequently the decrease in the percentage of these constituents is due to the rapid increase in the carbohydrates. The results obtained by Heinrich appear as follows when tabulated:

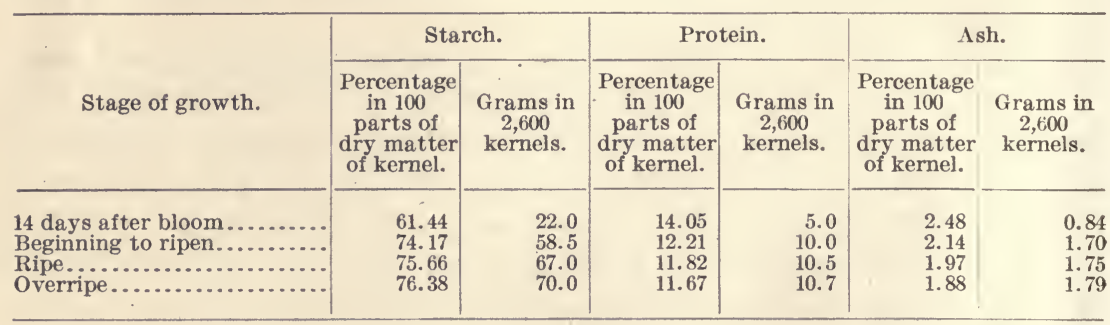

Nedokutschajew ${ }^{d}$ analyzed wheat kernels at different stages of development and found an almost uniform decrease in the percentage

$b$ Zusammensetzung $\mathrm{u}$. Verdaulichkeit der Futtermittel, 1, p. 419.

$c$ Handbuch des Getreidebaues, Berlin, 1884, 2, pp. 474-476.

$d$ Landw. Vers. Stat., 56 (1902), pp. 303-310. 
up at that time is, he says, used later for the development of the grain.

It is too well known to require substantiation by experimental evidence that the yield of grain per acre and the weight of the individual kernel increase as the grain approaches ripeness. It is therefore quite evident that immaturity, although resulting in a higher percentage of nitrogen in the wheat kernel, would curtail the production of nitrogen by the crop, and, furthermore, that the production of proteids would be still further lessened by reason of the greater proportion of amid substances present in the grain at that time.

\section{INFLUENCE OF IMMATURE SEED UPON YIELD.}

Georgeson ${ }^{a}$ selected kernels from wheat plants that were fully ripe, and from plants cut.while the grain was in the milk. He seeded these at the same rate on 2 one-tenth acre plots of land. The immature seed yielded at the rate of 19.75 bushels per acre of grain and 0.8 ton of straw, while the mature seed produced 22 bushels of grain and 1.04 tons of straw per acre. Georgeson says that in a similar experiment the previous year the difference in favor of the mature seed was still more pronounced.

Although the evidence is limited, it may safely be considered that the use of immature seed will result in a smaller yield of wheat than if fully ripe seed be used.

\section{'INFLUENCE OF CLIMATE UPON COMPOSITION AND YIELD.}

Lawes and Gilbert ${ }^{b}$ state that "high maturation in the wheat crop as indicated by the proportion of dressed corn in total corn, proportion of corn in total product (grain and straw), and heavy weight of grain per bushel, is, other things being equal, generally associated with a high percentage of dry substance and a low percentage of both mineral and nitrogenous constituents." This is based upon the wheat crops at Rothamsted for the years 1845 to 1854 , inclusive.

More recent publications ${ }^{c}$ by these investigators reaffirm their belief that the composition of the wheat kernel depends more largely upon the conditions that affect its degree of development than upon any other factor. They found almost invariably that a season that favored a long and continuous growth of the plant after heading, resulting in a large yield of grain, a high weight per bushel, and a plump kernel, produced a kernel of low nitrogen content.

a Abstract, Experiment Station Record, 4, p. 407, from Kansas Experiment Station Bulletin 33, p. 50 .

$b$ On Some Points in the Composition of Wheat Grain, London, 1857.

$c$ Our Climate and Our Wheat Crops, London, 1880, and On the Composition of the Ash of Wheat Grain and Wheat Straw, London, 1884. 
Körnicke and Werner ${ }^{a}$ cite an experiment in which winter wheat grown in Poppelsdorf for several years was sent to and grown in the moist climate of Great Britain, in Germany, and in the continental climate of Russia (steppes). The results were as follows:

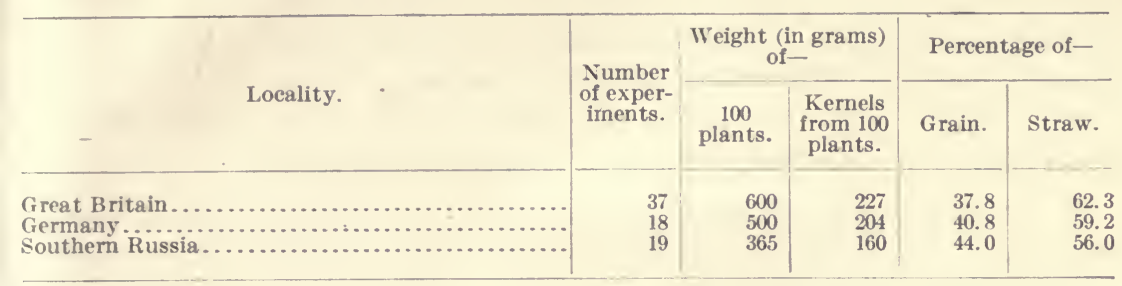

These investigators conclude from the results that in a moist climate relatively more straw and less grain are produced than in a dry, warm climate. The thickness of the straw and the weight of the kernels from 100 heads are greater, while the percentage by weight of kernels to straw is much less in a moist climate. They also quote Haberlandt as saying that a continental climate produces a small, hard wheat kernel, rich in gluten and of especially heavy weight,

Dehérain and Dupont ${ }^{b}$ report some interesting observations as to the effect of climate on the composition of wheat. They state that the harvest of 1888 at Grignon was late and the process of ripening slow. There was a heavy yield of grain having a gluten content of 12.60 per cent and a starch content of 77.2 per cent. The following season was dry and hot, with a rapid ripening of the grain, resulting in a smaller crop. The gluten content of the grain was 15.3 per cent and the starch content 61.9 per cent. They removed the heads from a number of plants. The next day the stems were harvested, as were also an equal number of entire plants. The stems without heads showed that carbohydrates equal to 5.94 per cent of the dry matter had been formed. The stems on which the heads remained one day longer contained 1.63 per cent carbohydrates. They argue from this that the upper portion of the stem, provided it is still green, performs the functions of the leaves in other plants and thus elaborates the starch that fills out the kernel in its later development.

A report from the Ploti Experiment Station ${ }^{c}$ states that the conditions that favored an increase in yield caused a reduction in the relative proportion of nitrogen in the grain. Excessive humidity favored the process of assimilation of carbohydrates, while drought hastened maturation and produced a grain relatively rich in proteids.

a Handbuch des Getreidebaues, Berlin, 1884, pp. 69, 70.

${ }^{b}$ Ann. Agron., 1902, p. 522.

c Abstract, Experiment Station Record, 14, p. 340, from Sept. Rap An. Sta. Expt. Agron. Ploty, 1901, pp. xiv-180. 
Wiley $^{a}$ sent wheat of the same origin to California, Kentucky, Maryland, and Missouri. The original grain and the product from each State were analyzed. The results of one year's test were reported. Regarding the effect of climate, he says:

There appears to be a marked relation between the content of protein matter and starch and the length of the growing season. The shorter the period of growth and the cooler the climate the larger the content of protein and the smaller the content of starch, and vice versa.

Shindler, ${ }^{b}$ in his book upon this subject, says (p. 75):

With the length of the growing period, especially with the length of the interval between bloom and ripeness, varies not only the size of the kernel, but also the relative amount of carbohydrates and protein it contains.

\section{Again, on page 76, Shindler says:}

All this shows that the protein constituent of the kernel depends in the first place upon the length of the growing period and next upon the richness of the soil.

Melikov ${ }^{c}$ made analyses of different varieties of wheat of the crops of the years 1885-1899 grown in southern Russia. The protein varied in different years from 14 to 21.2 per cent. Melikov concludes that the nitrogen content is highest in dry years and lowest in years of larger rainfall, in which years the yield of wheat per acre is also greater.

Gurney and Morris, ${ }^{d}$ in one of their reports, say:

This increased gluten [over previous years] is probably largely due to differences in the seasons, the weather being hot and dry while the grain was ripening, since it is characteristic not of these wheats alone but of most of the grain grown in the colony.

The conclusion to be inevitably derived from these observations is that climate is a potent factor in determining the yield and composition of the wheat crop, and, further, that its effect is produced by lengthening or shortening the growing season, particularly that portion of it during which the kernel is developing. A moderately cool season, with a liberal supply of moisture, has the effect of prolonging the period during which the kernel is developing, thus favoring its filling out with starch, the deposition of which is much greater at that time than is that of nitrogenous material. With this goes an increase in volume weight and an increased yield of grain per acre. On the other hand, a hot, dry season shortens the period of kernel development, curtails the deposition of starch, leaving the per-

$a$ Yearbook U. S. Department of Agriculture, 1901, pp. 299-308.

$b$ Der Weizen in seinem Beziehungen zum Klima und das Gesetz der Korrelation, Berlin, 1893.

$c$ Abstract, Experiment Station Record, 13, p. 451, from Zhur. Opuitn. Agron., 1 (1900), pp. 256-267.

$d$ Agricultural Gazette of New South Wales, 12, pt. 2, pp. 1403-1424. 
centage of nitrogen relatively higher, and gives a grain of lighter weight per bushel and smaller yield per acre.

The fact that one variety of wheat is adapted to a hot, dry climate and another to a cool, moist one does not mean that the former undergoes as complete maturation as the latter, even though the grain is not shriveled. This is shown by the fact that a variety of wheat well adapted to a hot, dry climate will, when planted in a cool, moist one, immediately grow plumper and the kernel weight will increase, as was the case in the experiment of taking Minnesota wheats to Maine.

\section{INFLUENCE OF SOIL UPON COMPOSITION AND YIELD.}

In considering the effect of the soil upon the wheat crop there will naturally be included experiments designed to show the effect of fertilizers upon the crops. It is, in fact, upon experiments with fertilizers that we must depend for most of our information on this subject.

Experiments to ascertain the effect of fertilizers upon the composition of the wheat kernel were conducted by Lawes and Gilbert for a period of years extending from 1845 to $1854 .^{a}$ Plots of land in which wheat was grown continually were treated annually as follows: Unmanured, manured with ammoniacal fertilizer alone, and manured with ammoniacal fertilizer and proportionate amounts of mineral salts. In composition calculated to dry matter, the wheat on the plots receiving ammoniacal fertilizer alone contained quite uniformly a slightly larger amount of nitrogen than either of the other two. The averages for the ten years were as follows:

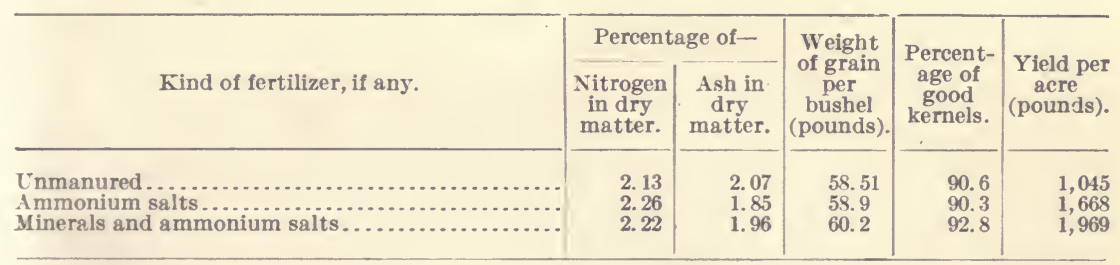

There was practically no difference in the nitrogen content of the straw. From these experiments the authors quoted conclude that there is no evidence that the nitrogen content of the wheat kernel can be increased at pleasure by the use of nitrogenous manures.

Ritthausen and Pott $^{b}$ report an experiment in which plots of land were manured (1) with superphosphate alone, (2) with nitrate alone, (3) with a mixture of superphosphate and nitrate, and (4) were left 
unmanured. There were three plots of each. The following is a tabulated statement of their results:

\begin{tabular}{|c|c|c|c|}
\hline Kind of fertilizer, if any. & $\begin{array}{l}\text { Weight of } \\
52 \text { c. c. of } \\
\text { kernels } \\
\text { (grams). }\end{array}$ & $\begin{array}{l}\text { Yield of } \\
\text { grain on } \\
\text { plot } \\
\text { (kilos). }\end{array}$ & $\begin{array}{l}\text { Percentage } \\
\text { of nitrogen } \\
\text { in dry } \\
\text { matter. }\end{array}$ \\
\hline 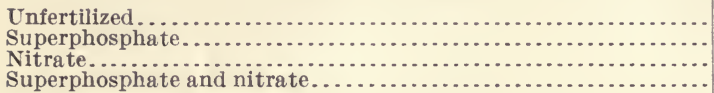 & $\begin{array}{l}1,306 \\
1,339 \\
1,413 \\
1,451\end{array}$ & $\begin{array}{l}2.72 \\
2.30 \\
2.03\end{array}$ & $\begin{array}{l}2.60 \\
3.49 \\
3.43 \\
3.62\end{array}$ \\
\hline
\end{tabular}

It will be noticed that the effect of the nitrate fertilizer was to decrease the yield of grain, but to increase the size of the kernel and its content of nitrogen.

Wolff, ${ }^{a}$ as early as 1856, in summing up the experiments of Hermbstadt, Muller, and John with barley, and of Lawes and Gilbert with wheat, says:

In the presence of a sufficient amount of phosphoric acid and alkali the effect of manuring with an easily soluble nitrogen compound is an improvement in the grain both in quantity and quality [meaning plumper kernels]. The kernels decrease in percentage of nitrogen, but become plumper, become absolutely and relatively richer in starch, and have a better appearance and a higher commercial value. But when the nitrogenous food in the soil exceeds a certain relation to the temperature and rainfall the quality of the grain becomes poorer [harder], it becomes lighter and smaller, takes on a darker color, and generally becomes richer in percentage of nitrogen in the air-dry substance.

Von Gohren ${ }^{b}$ also reports results of experiments in fertilizing wheat. All experiments were apparently made in the same year. He grew the crop on six different plots of land, five of which were manured and each with a different fertilizer. In the crop he distinguished between large kernels and small kernels to show the quality of the product. Determinations of proteids and starch were made, and these were calculated to the yield of each constituent on each plot.

The following table shows the yield of each of the characters determined, and compares those raised on the unmanured plot with those on the manured ones by taking the former as one and reducing the others to the corresponding figure:

\begin{tabular}{|c|c|c|c|c|c|c|}
\hline Yield and percentage. & $\begin{array}{l}\text { Unferti- } \\
\text { lized. }\end{array}$ & Ashes. & Oil cake. & $\begin{array}{c}\text { Bat } \\
\text { guano. }\end{array}$ & $\begin{array}{l}\text { Oil cake } \\
\text { and } \\
\text { ashes. }\end{array}$ & $\begin{array}{l}\text { Peruvian } \\
\text { guano. }\end{array}$ \\
\hline 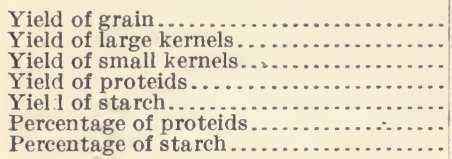 & $\begin{array}{r}1.000 \\
1.000 \\
1.000 \\
1.000 \\
1.000 \\
14.42 \\
62.67\end{array}$ & $\begin{array}{r}1.011 \\
.146 \\
.953 \\
.999 \\
1.009 \\
14.25 \\
62.56\end{array}$ & $\begin{array}{r}1.071 \\
1.928 \\
.704 \\
.915 \\
1.081 \\
12.70 \\
63.25\end{array}$ & $\begin{array}{r}1.143 \\
2.552 \\
.538 \\
.936 \\
1.174 \\
11.81 \\
64.41\end{array}$ & $\begin{array}{l}1.215 \\
2.225 \\
.781 \\
1.070 \\
1.264 \\
12.70 \\
65.24\end{array}$ & $\begin{array}{r}1.286 \\
2.786 \\
.642 \\
1.114 \\
1.303 \\
13.22 \\
63.55\end{array}$ \\
\hline
\end{tabular}

The results show an increased yield from the use of fertilizers, the production increasing with the application of complete manures.

a Die naturgesetzlichen Grundlagen des Ackerbaues, Leipzig, 1856, p. 774.

$b$ Landw. Vers. Stat., 6 (1864), pp. 15-19. 
The yield of grain of good quality increases in the same way, and the yield of grain of poor quality decreases proportionately. It must be remembered that by good quality of grain in these early writings is meant plump kernels and not necessarily what would be considered wheat of good milling quality at the present day. The production of proteids per acre decreased with the use of the incomplete fertilizers, ashes and oil cake, and even with the bat guano. It increased, however, with the use of oil cake and ashes combined and of Peruvian guano. The percentage of proteids was greatest in the unfertilized grain and the percentage of starch least, with the exception of one fertilized plot.

The very evident effect of the fertilizers in this case was to produce a more completely matured kernel. It will be noticed that the plots producing grain of highest starch content were those having the greatest proportion of plump kernels.

Again, in 1884, Lawes and Gilbert ${ }^{a}$ report results obtained from manured and unmanured soils. These experiments cover a period of sixteen years and are divided into two periods of eight years each. In one of these periods the seasons were favorable for wheat, in the other unfavorable.

\begin{tabular}{|c|c|c|c|c|c|c|}
\hline \multirow[b]{2}{*}{ Character. } & \multicolumn{3}{|c|}{ Favorable seasons. } & \multicolumn{3}{|c|}{ Unfavorable seasons. } \\
\hline & $\begin{array}{l}\text { Barnyard } \\
\text { manure. }\end{array}$ & $\begin{array}{l}\text { Un- } \\
\text { manured. }\end{array}$ & $\begin{array}{l}\text { Ammo- } \\
\text { nium salts } \\
\text { alone. }\end{array}$ & $\begin{array}{l}\text { Barnyard } \\
\text { manure. }\end{array}$ & $\begin{array}{c}\text { Un- } \\
\text { manured. }\end{array}$ & $\begin{array}{l}\text { Ammo- } \\
\text { nium salts } \\
\text { alone. }\end{array}$ \\
\hline 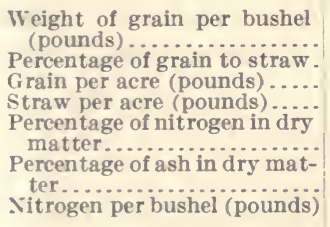 & $\begin{array}{r}62.6 \\
62.5 \\
2,342.0 \\
6,089.0 \\
1.73 \\
1.98 \\
1.083\end{array}$ & $\begin{array}{r}60.5 \\
67.4 \\
1,156.0 \\
2,872.0 \\
1.84 \\
1.96 \\
1.113\end{array}$ & $\begin{array}{r}60.4 \\
66.2 \\
1,967.0 \\
4,774.0 \\
2.09\end{array}$ & $\begin{array}{r}57.4 \\
54.5 \\
1,967.0 \\
5,574.0 \\
1.96 \\
\\
2.06 \\
1.125\end{array}$ & $\begin{array}{r}54.3 \\
51.1 \\
823.0 \\
2,433.0 \\
1.98 \\
\\
2.08 \\
1.075\end{array}$ & $\begin{array}{r}53.7 \\
46.7 \\
1,147.0 \\
3,601.0 \\
2.25 \\
1.91 \\
1.208\end{array}$ \\
\hline
\end{tabular}

It is evident from this statement that the largest crops and best developed kernels were obtained from the soils treated with barnyard manure, and that these kernels contained the lowest percentage of nitrogen. The crops on unmanured soil stood next in these respects, except in yield. Those on the soil receiving ammonium salts produced the most poorly developed kernels and those of highest nitrogen content, but gave larger yields than the unmanured soil.

In the unmanured soil there was a very evident lack of plant food, as indicated by the light crops. The effect upon the kernel was to curtail its development, leaving it of light weight and with a relatively high nitrogen content.

$a$ On the Composition of the Ash of Wheat Grain and Wheat Straw, London, 1884. 
Hermbstadt obtained some curious results, as quoted by D.G.F. MacDonald, ${ }^{a}$ as follows:

He sowed equal quantities of wheat upon the same ground and manured them with equal weights of the different manures set forth below. From 100 parts of each sample of grain produced he obtained starch and gluten in the following proportions:

\begin{tabular}{|c|c|c|c|}
\hline Kind of fertilizer, if any. & Gluten. & Starch. & Produce. \\
\hline 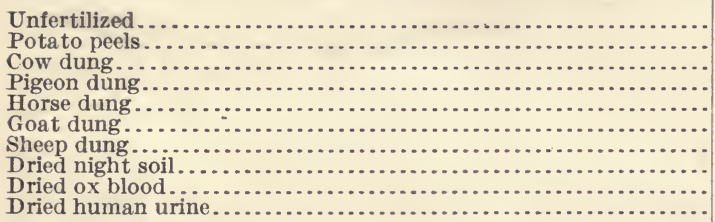 & $\begin{array}{l}9.2 \\
9.6 \\
12.0 \\
12.2 \\
13.7 \\
32.9 \\
32.9 \\
33.14 \\
34.24 \\
31.1\end{array}$ & $\begin{array}{l}66.7 \\
65.94 \\
62.3 \\
63.2 \\
61.64 \\
42.4 \\
42.8 \\
41.44 \\
41.43 \\
39.3\end{array}$ & $\begin{array}{l}\text { Threefold. } \\
\text { Fivefold. } \\
\text { Sevenfold. } \\
\text { Ninefold. } \\
\text { Tenfold. } \\
\text { Twelvefold. } \\
\text { Do. } \\
\text { Fourteenfold. } \\
\text { Do. } \\
\text { Twelvefold. }\end{array}$ \\
\hline
\end{tabular}

These results are not to be considered seriously, representing as they do an impossible condition.

Prof. H. A. Huston ${ }^{b}$ treated 0.01-acre plots of land each with nitrate of soda, dried blood, sulphate of ammonia, rotted stable manure, and muck, respectively, either in the autumn or spring, or in both seasons. In 1891 all the plots treated with nitrogenous compounds showed marked increase in the percentage of nitrogen in the grain. In 1892 the results were by no means so uniform and would not justify the conclusion that nitrogenous fertilizers increased the nitrogen content of the wheat.

Vignon and Conturier ${ }^{c}$ tested the effect of phosphate fertilizer alone upon the nitrogen content of the grain of two varieties of wheat. On Plot 1 they used 75 kilograms of phosphoric acid per hectare; on Plot 2, 150 kilograms, and on Plot 3, 225 kilograms.

\begin{tabular}{|c|c|c|c|}
\hline \multirow{2}{*}{ Variety. } & \multicolumn{3}{|c|}{$\begin{array}{l}\text { Percentage of nitrogen in } \\
\text { grain. }\end{array}$} \\
\hline & Plot 1. & Plot 2. & Plot 3. \\
\hline 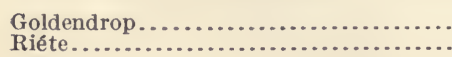 & $\begin{array}{l}1.83 \\
2.07\end{array}$ & $\begin{array}{l}1.61 \\
1.98\end{array}$ & $\begin{array}{l}1.54 \\
1.82\end{array}$ \\
\hline
\end{tabular}

There was a very evident decrease in the nitrogen content of the crop as the quantity of fertilizer was increased.

It was concluded from experiments conducted at the Ploti Experiment Station ${ }^{d}$ that, with favorable meteorological conditions, manure increased the total amount of nitrogen taken up by wheat, but,

$a$ Practical Hints on Farming, London, 1868.

$b$ Indiana Experiment Station Bulletins 41 and 45.

${ }^{c}$ Compt. Rend., 132 (1901), p. 791.

$d$ Abstract, Experiment Station Record, 14, p. 340, from Sept. Rap. An. Sta. Expt. Agron. Ploty, 1901, pp. xiv-180. 
although it thus increased the total production of nitrogen, it decreased the relative proportion of nitrogenous substance.

Bogdau ${ }^{a}$ conducted investigations the results of which indicated that with an increase in the soluble salt content of 22 alkali soils the nitrogen and ash contents of the wheat kernels increased, but the absolute weight of the kernels diminished. These soluble salts are rich in nitrates.

Experiments were conducted by Whitson, Wells, and Vivian ${ }^{b}$ in which plants were grown in pots the soils of which were in some cases fertilized with nitrates and in others with leachings of single and of double strengths from fertile soils. Field experiments were conducted on manured and unmanured plots. All of the analyses, except in the case of oats, were of the whole plant. Of the ripe oat kernels those from the unfertilized soil contained 2.57 per cent of nitrogen, while the average of those from the fertilized soil was 2.78 per cent.

Guthrie $^{c}$ conducted experiments with fertilizers for wheat during two years, in which he kept a record of the yield and gluten content of the grain. The following is a statement of the results:

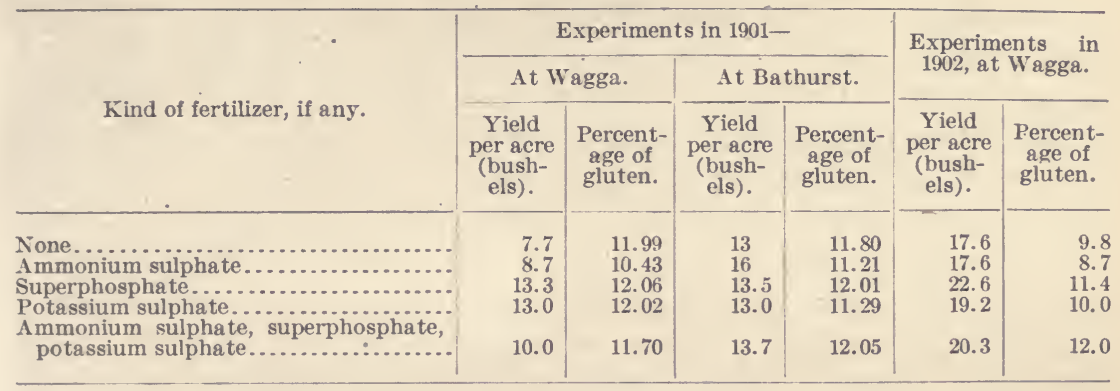

In this experiment there was in each case a higher percentage of gluten in the wheat raised on the fertilized soil than in that from the soil fertilized with ammonium sulphate, and in the latter less than in the grain fertilized with other material.

The most striking feature of these results is their apparent lack of uniformity. In some cases the use of nitrogenous fertilizers was accompanied by an increase in the nitrogen content of the grain and in other cases no increase appeared; in some cases phosphoric acid fertilizers apparently increased the nitrogen content and in others they did not have this effect.

Climatic influences have doubtless operated largely in these results, but they are not considered by any of the experimenters except Wolff.

"Abstract, Experiment Station Record, 13, p. 329, from Report of Department of Agriculture, St. Petersburg, 1500.

$\checkmark$ Wisconsin Experiment Station Report, 19 (1902), pp. 192-209.

c Agricultural Gazette of New South Wales, 13 (1902), No. 6, p. 664; and No. 7, p. 728. 
It is evident that in all experiments with depleted soils the plants on the plots receiving complete fertilizers would take up larger amounts of plant food, including nitrogen, than would plants on unmanured soils. Any conditions that would prevent the normal ripening of the crop on both soils would therefore leave a higher percentage of nitrogen in the plants upon the unmanured soil. On the other hand, under conditions which would permit of a complete maturation of the crop there might be no difference in the composition of the grain from the manured and unmanured soils. It is evident, however, that the production of both nitrogen and starch in pounds per acre would be greater on the manured soils.

Another condition that may affect the results is the arrested development of kernels on unmanured soils that are seriously depleted of plant food. Such depletion may interfere with complete maturation of the crop while the crop on the manured soil will mature fully. In consequence the grain on the unmanured soil will contain a higher percentage of nitrogen but a smaller yield per acre. The use of a nitrogenous manure alone on exhausted soils may likewise result in a grain of higher nitrogen content.

Expressed in a more general way, this means that wheat of the same variety grown under the same climatic conditions will have approximately the same percentage of nitrogen if allowed to mature fully, but any permanent interruption in the process of maturation will result in a higher percentage of nitrogen, and in the latter case the percentage of nitrogen will depend upon the stage at which development was interrupted, and also upon the amount of nitrogen accumulated by the plant, that being greater on soils manured with nitrogenous fertilizers alone than on exhausted soils, and greater on soils receiving complete manures than on exhausted soils receiving only nitrogenous fertilizers, provided the stage at which development ceased be the same in both cases. It thus happens that wheat growing on the soil allowing it to absorb the largest amount of nitrogen will, other things being equal, have a higher nitrogen content if the development of the kernel be permanently checked, although if it were allowed to mature fully it would not have a greater percentage of nitrogen than that grown on the soil affording less nitrogen.

Reviewing the experiments, we find that in Lawes and Gilbert's first experiment the percentage of nitrogen in the unmanured soil was less than on the soil receiving only nitrogenous fertilizer, and that the weight of grain per bushel and the percentage of good kernels on the two plots were practically the same. It would not appear, therefore, that the wheat on the plot receiving the nitrogenous fertilizer was less well matured than that on the unmanured plot. In this case there appears to be a slight increase in the percentage of nitrogen, due entirely to the use of nitrogenous fertilizers. Comparing the grain on 
the plot receiving only nitrogenous fertilizer with that receiving the complete fertilizer it will be seen that the former has a higher percentage of nitrogen, but this is evidently due to the poorly developed kernels which weigh less per bushel than the grain on the completely fertilized plot.

Von Gohren's results show plainly that the kernels on the manured land developed better than on the unmanured, and with this better development there was an increase in the percentage of starch and a decrease in the nitrogen.

In Lawes and Gilbert's second experiment the percentage of nitrogen in the wheat on the soil manured with ammonium salts was less than that in the wheat on the unmanured soil, but the weight of grain per bushel shows that the higher nitrogen content was due, in part at least, to incomplete maturation. The higher percentage of nitrogen in the wheat on the soil receiving only nitrogenous manures as compared with that receiving complete manures can be traced to the same condition of the grain.

INFLUENCE OF SOIL MOISTURE UPON COMPOSITION AND YIELD.

Experiments were conducted by D. Prianishinkov ${ }^{a}$ in which wheat was raised with different degrees of moisture, but in the same soil and under the same conditions of light and temperature. With a larger amount of moisture in the soil there was a lower nitrogen content in the grain. It was also stated that the duration of the period of vegetation was somewhat shorter when the moisture supply was greater.

Traphagen ${ }^{b}$ reports marked changes in the composition of wheat grown with and without irrigation at the Montana Experiment Station. A wheat grown under irrigation on the station farm was planted the following year on land not irrigated. Presumably the land was of similar character. The two crops of grain were analyzed and the percentages stated below were found.

\begin{tabular}{|c|c|c|c|c|c|c|}
\hline Crop. & $\begin{array}{l}\text { Mois- } \\
\text { ture. }\end{array}$ & $\begin{array}{l}\text { Crude } \\
\text { protein. }\end{array}$ & $\begin{array}{l}\text { Ether } \\
\text { extract. }\end{array}$ & $\begin{array}{c}\text { Nitrogen- } \\
\text { free } \\
\text { extract. }\end{array}$ & $\begin{array}{l}\text { Crude } \\
\text { fiber. }\end{array}$ & Ash. \\
\hline $\begin{array}{l}\text { Irrigated wheat } \ldots \ldots \ldots \ldots \ldots \ldots \\
\text { Unirrigated wheat } \ldots \ldots \ldots \ldots \ldots \ldots\end{array}$ & $\begin{array}{r}\text { Per ct. } \\
7.87 \\
7.65\end{array}$ & $\begin{array}{r}\text { Per ct. } \\
8.81 \\
14.41\end{array}$ & $\begin{array}{r}\text { Per ct. } \\
1.93 \\
2.23\end{array}$ & $\begin{array}{r}\text { Per ct. } \\
76.99 \\
71.33\end{array}$ & $\begin{array}{r}\text { Per ct. } \\
2.60 \\
2.65\end{array}$ & $\begin{array}{r}\text { Per ct. } \\
1.80 \\
1.70\end{array}$ \\
\hline
\end{tabular}

No records of yields or of weights of kernels are given, but it is fair to suppose that the unirrigated wheat possessed the light, shrunken kernel which is characteristic of wheat raised without sufficient moisture.

"Abstract, Experiment Station Record, 13, p. 631, from Zhur. Gpuitn. Agron., 1 (1900), No. 1, pp. 13-20.

${ }^{b}$ Montana Experiment Station Report (1902), pp. 59-60. 
Irrigation experiments were conducted by Widtsoe ${ }^{a}$ in which wheat of the same variety was raised on plots of land each one of which received a different quantity of water. A record was kept of the yield and composition of the grain on each plot.

\begin{tabular}{|c|c|c|c|c|c|c|}
\hline \multirow[b]{2}{*}{ Plot. } & \multirow{2}{*}{$\begin{array}{l}\text { Water } \\
\text { applied } \\
\text { (inches). }\end{array}$} & \multirow{2}{*}{$\begin{array}{l}\text { Yield } \\
\text { per acre } \\
\text { (bush- } \\
\text { els). }\end{array}$} & \multicolumn{2}{|c|}{ Percentage of- } & \multicolumn{2}{|c|}{$\begin{array}{l}\text { Yield (in pounds) } \\
\text { per acre of }-\end{array}$} \\
\hline & & & $\begin{array}{l}\text { Protein } \\
\text { in grain. }\end{array}$ & $\begin{array}{l}\text { Ash in } \\
\text { grain. }\end{array}$ & Nitrogen. & Ash. \\
\hline 317 & 4.63 & 4.50 & 24.8 & 2.50 & 10.7 & 6.75 \\
\hline 319 & 5.14 & 3.83 & 23.2 & 3.07 & 8.5 & 7.05 \\
\hline 320 & 8.73 & 10.33 & 19.9 & 2.54 & $\begin{array}{l}0.0 \\
19.7\end{array}$ & 15.74 \\
\hline 318 & 8.89 & 11.33 & 19.4 & 2.93 & 21.1 & 19.72 \\
\hline 321 & 10.30 & 14.66 & 18.4 & 2.34 & 25.9 & 20.24 \\
\hline 325 & 12.09 & 11.16 & 21.3 & 3.25 & 22.8 & 21.44 \\
\hline 322 & 12.18 & 11.66 & 23.1 & 2.88 & 25.8 & 20.30 \\
\hline 326 & 12.80 & 13.00 & 17.1 & 2.52 & 21.3 & 21.50 \\
\hline 327 & 17.50 & 15.33 & 17.2 & 2.57 & 25.3 & 23.64 \\
\hline 328 & 21.11 & 17.33 & 15.9 & 2.34 & 26.4 & 24.33 \\
\hline 329 & 30.00 & 26.66 & 14.0 & 4.14 & 35.8 & 66.20 \\
\hline 330 & 40.00 & 14.50 & 17.1 & 2.52 & 23.8 & 21.92 \\
\hline
\end{tabular}

The results show that with an increase in the water used for irrigation up to 30 inches there were in general an increase in the yield of grain and a decrease in the nitrogen content. No volume weights or other means of judging of the development of the kernels on the different plots are given, but there is no reason to suppose that the grain on the plots receiving small quantities of water was not poorly developed. The column added showing the yield of nitrogen in pounds per acre indicates a lack of nutriment in the grain on these plots. $^{b}$

High nitrogen content arising from a small supply of soil moisture is sometimes due to a restricted development of the kernel. There is nothing in these results to indicate a greater absorption of nitrogen by the crop on soil having less moisture, but results of this nature are cited elsewhere in this bulletin.

INFLUENCE OF SIZE OR WEIGHT OF THE SEED-WHEAT KERNEL UPON THE CROP YIELD.

Sanborn ${ }^{c}$ reports experiments to ascertain the effect of separating seed wheat into kernels of different grades to ascertain the effect upon the yield. He divided the kernels into large, medium, small, ordinary (grain as it came from the thrasher), and shriveled, and continued the experiments for four years. Apparently the large kernels were separated from the crop grown from large seed the previous year, and

$a$ Utah Experiment Station Bulletin 80.

${ }^{b}$ Nitrogen has been calculated from proteids by dividing by 6.25 .

$c$ Utah Experiment Station Report, 1893, p. 168. 
so with the other classes of kernels. He tabulates his results as follows:

\begin{tabular}{|c|c|c|c|c|c|}
\hline \multirow{2}{*}{ Kind of seed. } & \multicolumn{4}{|c|}{$\begin{array}{c}\text { Yield of grain on plots (in } \\
\text { pounds) }\end{array}$} & \multirow{2}{*}{$\begin{array}{c}\begin{array}{c}\text { Average } \\
\text { for } 4 \\
\text { years. }\end{array} \\
\begin{array}{c}\text { Bushels } \\
\text { per acre. }\end{array}\end{array}$} \\
\hline & 1890. & 1891. & 1892. & 1893. & \\
\hline
\end{tabular}

The relation between yields of the crops representing different. sized kernels is so irregular from year to year that suspicion is aroused regarding the accuracy of the results, due to lack of uniformity in soil. Sanborn's conclusion is that very little, if any, advantage is to be gained by separating seed wheat and planting the large kernels.

At the Indiana Experiment Station, Latta ${ }^{a}$ conducted experiments in which wheat was separated by means of a fanning mill into heavy and light kernels, but impurities and chaffy seed were fanned out of each lot of wheat. The experiments were continued three years, but the separations were made each year from seed that had not been so separated the year before. The average gain from the large seed for three years was 2.5 bushels per acre.

Georgeson, ${ }^{b}$ at the Kansas station, seeded plots of land with (1) light seed weighing 56 pounds per bushel, (2) common seed weighing 62.5 pounds, (3) heavy seed weighing 63 pounds, and (4) selected seed, obtained by picking the largest and finest heads in the field just before the crop was cut, weighing 61.5 pounds per bushel. Seed was separated each year from wheat not grown from previously selected seed. The average results for three years were as follows:

\begin{tabular}{|c|c|c|c|}
\hline Grade of seed. & \begin{tabular}{|c} 
Yield of \\
grain \\
per acre \\
(bush- \\
els).
\end{tabular} & Grade of seed. & $\begin{array}{l}\text { Yield of } \\
\text { grain } \\
\text { per acre } \\
\text { (bush- } \\
\text { els). }\end{array}$ \\
\hline $\begin{array}{l}\text { Light.......... } \\
\text { Common..... }\end{array}$ & $\begin{array}{l}25.19 \\
26.57\end{array}$ & 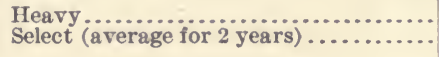 & $\begin{array}{l}27.07 \\
25.82\end{array}$ \\
\hline
\end{tabular}

Desprez $^{c}$ reports experiments extending through three years in which large kernels were selected from a crop grown from large seed

$a$ Indiana Experiment Station Bulletin 36, pp. 110-128.

${ }^{b}$ Kansas Experiment Station Bulletin 40, pp. 51-62.

${ }^{c}$ Abstract, Experiment Station Record, 7, p. 679, from Jour. Agr. Prat., 59 (1895), 2, pp. 694-698. 
for several years and small seed from a crop grown from small seed for several years. Five varieties of wheat were used. The average results for three years were a difference of 1,067 to 1,828 kilograms of grain per hectare in favor of the large seed, but the difference was in general greater the first year than later. The use of large seed gave a crop with kernels larger than those grown from small seed.

Middleton ${ }^{a}$ reports the yields obtained from large wheat kernels to be almost double those obtained from small seed kernels.

Bolley, ${ }^{b}$ as the results of experiments continuing for four years in which plump kernels of large size and plump kernels of small size were selected for seed, concludes that "perfect grains of large size and greatest weight produce better plants than perfect grains of small size and light weight, even when the grains come from the same head."

At the Ontario Agricultural College, Zavitz' ${ }^{c}$ selected large plump seed, small plump seed, and shrunken seed of both spring and winter wheat. Experiments were continued for eight years with spring wheat and five years with winter wheat, the selections each year being from a crop grown from previously unselected seed. His results are as follows:

\begin{tabular}{|c|c|c|}
\hline \multirow{2}{*}{ Kind of seed. } & \multicolumn{2}{|c|}{$\begin{array}{l}\text { Yield per acre (in } \\
\text { tushels). }\end{array}$} \\
\hline & $\begin{array}{l}\text { Spring } \\
\text { wheat. }\end{array}$ & $\begin{array}{l}\text { Winter } \\
\text { wheat. }\end{array}$ \\
\hline $\begin{array}{l}\text { Large, plump.............. } \\
\text { Small, plump ............. } \\
\text { Shrunken.............. }\end{array}$ & $\begin{array}{l}21.7 \\
18.0 \\
16.7\end{array}$ & $\begin{array}{l}42.4 \\
34.8 \\
33.7\end{array}$ \\
\hline
\end{tabular}

Dehérain and Dupont ${ }^{d}$ report that the yields from small and large kernels of a number of varieties of wheat were in all cases in favor of the large kernels, but a large difference in yield was obtained only when there was a marked difference in the weight of the kernels.

Soule and Vanatter ${ }^{e}$ conducted experiments for three years in which large and small kernels were separated by means of sieves. In addition a plot of unselected seed was planted. The large seed was, each year after the first, selected from the crop grown from large seed the previous year. The same was true of the small seed. These investigators say:

a Abstract, Experiment Station Record, 12, p. 441, from Univ. Coll. of Wales Rept., 1899, pp. 68-70.

$b$ North Dakota Experiment Station Report, 1901, p. 30.

$c$ Ontario Agricultural College and Experiment Farm Report, 1901, p. 84.

$d$ Abstract, Experiment Station Record, 15, p. 672, from Compt. Rend., 135 (1902), p. 654 .

e Tennessee Experiment Station Bulletin, vol. 16, No. 4, p. 77. 
The average difference in yield at the end of three years between large grains ( 607 per ounce), commercial sample (689 per ounce), and small grains (882 per ounce), with Mediterranean wheat, was 2.06 bushels in favor of large grains as compared with the commercial sample, and 5.18 bushels in favor of large grains over small grains. The difference in yield between the large grains and the commercial sample chiefly occurred the first year; but it is possible, though hardly probable, that the difference was partly due to variation in the soil. The experiment has been carried on in different parts of the field for the last two years, and the difference in yield is now only 0.32 bushel per acre in favor of the large grains.

$\mathrm{Cobb}^{a}$ reports tests of various grades of wheat kernels with respect to size, and concludes that large kernels give better yields of grain. The seed of one year was not the product of the corresponding grade of the previous one.

Grenfell $^{b}$ selected plump and shriveled kernels from the same bulk of grain. Of these 150 kernels were sown in each row, with rows of plump and shriveled kernels alternating. The germination in both rows appeared much alike, but the plants in the rows sown from plump grain soon began to gain on the others and kept ahead for the remainder of the season. The tillering was better in the plumpgrain plants. Grenfell tabulates his results thus:

\begin{tabular}{|c|c|c|c|c|c|}
\hline Variety. & Kind. & $\begin{array}{l}\text { Percentage } \\
\text { of plants } \\
\text { that grew. }\end{array}$ & $\begin{array}{l}\text { Number } \\
\text { of heads. }\end{array}$ & $\begin{array}{l}\text { Tillering } \\
\text { power. }\end{array}$ & \begin{tabular}{|c} 
A verage \\
yield per \\
acre \\
(bush- \\
els).
\end{tabular} \\
\hline \multirow[t]{2}{*}{ 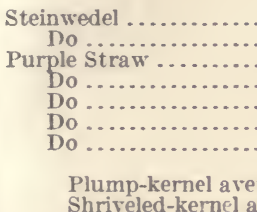 } & $\begin{array}{l}\text { Plump....... } \\
\text { Shriveled.... } \\
\text { Plido........ } \\
\text { Phimp...... } \\
\text { Shriveled.... } \\
\text { Plump...... } \\
\text { Shriveled.... }\end{array}$ & $\begin{array}{l}96.0 \\
89.3 \\
89.3 \\
90.0 \\
76.0 \\
92.0 \\
98.0\end{array}$ & $\begin{array}{l}179 \\
174 \\
153 \\
200 \\
140 \\
161 \\
155\end{array}$ & $\begin{array}{l}1.24 \\
1.29 \\
1.14 \\
1.49 \\
1.16 \\
1.23 \\
1.34\end{array}$ & $\begin{array}{r}10.9 \\
9.9 \\
6.1 \\
10 \\
6.9 \\
8.4 \\
7.2\end{array}$ \\
\hline & & $\begin{array}{l}92.7 \\
88.5\end{array}$ & $\begin{array}{l}180 \\
155\end{array}$ & $\begin{array}{l}1.32 \\
1.23\end{array}$ & $\begin{array}{l}9.8 \\
7.5\end{array}$ \\
\hline
\end{tabular}

As bearing upon this subject some experiments conducted by Rünker $^{c}$ are of interest. He weighed each of the kernels of a large number of heads of wheat of the Spalding Prolific and Martin Amber varieties, and found that the. heaviest kernels occur in the lower half of the spike. With spikes of different lengths and weights, the weight of the average kernel increases with the size of the spike.

Weights of individual kernels from the same spikes show that there is a great range in this respect. One spike, of which Rünker gives the weights of all the kernels, and which is given as representative of the average, shows kernels varying in weight from 36 to 71 milligrams.

a Agricultural Gazette of New South Wales, 14 (1903), No. 2, pp. 145-169.

b Agricultural Gazette of New South Wales, 12 (1901), No. 9, pp. 1053-1062.

c Jour. f. Landw., 38 (1890), p. 309.

27889 - No. $78-05-3$ 
It is therefore quite evident that a sample of wheat taken from spikes of different sizes when separated into lots of light and heavy kernels would have both the larger spikes and smaller spikes represented in each lot of kernels, but doubtless the proportion of kernels from large heads would be greater in the lot of heavy kernels.

It would appear from these results that the evidence was overwhelmingly in favor of large or heavy wheat kernels for seed. Most of the experimenters selected seed of different kinds each year without reference to previous selection. If large seed or small seed represent plants of different characteristics and if these properties are hereditary, the results of selection of large or small seeds for several years may be quite different from what they would be the first year. It is only those experiments in which selection of the same kind of seed has been continued for several generations that may be relied upon to indicate the value of continuous selection of large kernels for seed.

Such experiments have been conducted by Sanborn, by Desprez, and by Soule and Vanatter. The work of Desprez indicates that the size of the kernel is a hereditary quality. That being the case, it is evident that the small seed of the first separation may be composed partly of seed that is small on account of immaturity and partly of seed that is small by inheritance, but which is perfectly normal. When such seed is planted the immature seed will be largely eliminated in the crop, but the naturally small seed will have reproduced itself and will compose most of the crop. When the seed is again separated a much smaller percentage of small seed will be immature, and in consequence a larger number of kernels will produce plants. It would appear from Desprez's experiments, however, that those plants producing small kernels are not so prolific as those producing large kernels.

Sanborn's results make a very good showing for the small kernels, but, as before stated, the extreme irregularity would lead to the belief that the soil on the plots lacked uniformity, or that some other errors had influenced the results. To offset this the tests cover a period of four years, which should help to rectify mistakes, and in consequence the good showing made by the small kernels is entitled to some consideration.

Soule and Vanatter's results fulfill exactly the conditions of the hypothesis that the small seed would the first year contain a much larger proportion of immature kernels than it would in subsequent years, and hence yield more poorly the first year. Their results with heavy kernels as compared with ordinary seed offer little encouragement to the continuous selection of large kernels. 
The fact before referred to that both large and small kernels are found on the same head of wheat is perhaps an argument against the superior value of large seed. If the plant and not the seed is the unit of reproduction, small seèd from a plant whose kernels averaged large size may be better than large seed from a plant whose kernels averaged small size.

On the other hand, there can be no doubt that the majority of the kernels in the lot of heavy kernels would be from plants having large spikes, and vice versa. This would give the kernels in the heavy lot some advantage. Again, the advantage that the large kernel is supposed to possess for seed may not be in producing a large kernel in the resulting crop, but in giving the plant a better start in life, or producing a more vigorous plant.

\section{RELATION OF SIZE OF KERNEL TO NITROGEN CONTENT.}

Richardson ${ }^{a}$ has made a large number of analyses of wheats from different parts of the United States. The weight of 100 kernels was also determined in each sample. There can not be said to be any constant relation between the nitrogen content and the kernel weight, but in the main the large kernels have a lower percentage of nitrogen than the small kernels, and inversely.

Pagnoul $^{b}$ reports that in a test of eleven varieties of wheat there was in the main a decrease in the percentage of nitrogen in the crop as compared with the seed when there was an increase in the weight of 1,000 kernels in the crop as compared with the seed.

The same investigator ${ }^{c}$ again states that in an examination of seventy varieties of wheat there was no constant relation between the size of the kernels and their nitrogen content, but that in general the varieties with small kernels were the varieties richest in nitrogen.

Marek $^{l}$ separated wheat of the same variety into lots of large and of small kernels. He found on analysis that the large kernels contained 12.52 per cent protein and the small kernels 13.55 per cent protein.

Woods and Merrille made analyses of a number of wheats grown in Minnesota and of the same varieties grown in Maine. The wheats uniformly developed a larger kernel when grown in Maine. Grouping five varieties raised in Minnesota and five raised in Maine, it will be seen that with this increase in the size of the kernel there was a

a U. S. Department of Agriculture, Division of Chemistry, Bulletins 1 and 3.

$b$ Abstract in Centrlb. f. Agr. Chem., 1893, p. 616, from Ann. Agron., 1892, p. 486.

c Abstract in Centrlb. f. Agr. Chem., 1888, p. 767, from Ann. Agron., 14, pp. 262-272.

"Abstract in Centrlb. f. Agr. Chem., 1876, from Landw. Zeitung f. Westfalen u. Lippe, 1875, p. 362.

$\epsilon$ Maine Experiment Station Bulletin 97. 
decrease in the nitrogen content. The analyses, reduced to a waterfree basis, are as follows:

Where grown.

Weight of Percentage 100 kernels $\begin{aligned} & \text { Percentage } \\ & \text { of protein. }\end{aligned}$

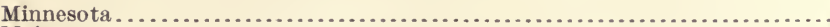

Maine...

In a review of the experiments concerning the relation of weight to composition of cereals, Gwallig" says that the results obtained by Marek, Wollny, Märcker, Hoffmeister, and Nothwang divide barley and rye into one group, and wheat and oats into another, as regards this relation. With barley and rye, the largest, heaviest kernels are the richest in protein. With wheat and oats, the smallest, lightest kernels have the highest protein content.

Gwallig says further that with an increased protein content there is a decrease in nitrogen-free extract. The fat and ash do not stand in a definite relation to the kernel weight, but the small, light kernels have a higher percentage of crude fiber, which circumstance is accounted for by the larger surface possessed by the smaller kernels.

Snyder ${ }^{b}$ has divided small kernels into two classes-those which are small because shrunken and those which are small although well filled. He finds that as between small kernels of the first class and large, well-filled kernels, the former contain a higher percentage of nitrogen, but as between the small, well-filled and the large, well-filled kernels, the latter contain the higher percentage of nitrogen. In testing this he used large and small kernels of the same variety in each case, and the wheats represented a large portion of the wheatgrowing area of the United States. As regards the relation of large, perfect, and small, perfect kernels there were twenty-four out of twenty-seven cases in which the large kernels contained a greater percentage of nitrogen.

Johannsen and Weis," in experiments with five.varieties of wheat, find that as a general rule the percentage of nitrogen is increased with increasing grain weight, but that there are many exceptions to the rule.

$\mathrm{Cobb}^{\prime \prime}$ states that small wheat kernels contain a larger proportion of gluten than do large ones, but he does not submit any analyses to substantiate his statement.

"Abstract in Centrlb. f. Agr. Chem., 24 (1895), p. 388, from Landw. Jahrbücher, 23 (1834), p. 835.

b) Mínnesota Experiment Station Bulletin 85.

${ }^{c}$ Abstract, Experiment Station Record, 12, p. 327, from Tidsskr. Landbr. Planteavl., 5 (1899), pp. 91-100.

$d$ Agricultural Gazette of New South Wales, 5 (1894), No. 4, pp. 239-250. 
Körnicke and Werner ${ }^{a}$ quote the experiments of Reiset to show that shriveled kernels have a higher nitrogen content than plump ones. With different varieties of wheat he found the following:

\begin{tabular}{|c|c|c|}
\hline Variety. & Kind. & $\begin{array}{l}\text { Percent- } \\
\text { age of } \\
\text { nitrcgen } \\
\text { in dry } \\
\text { matter. }\end{array}$ \\
\hline 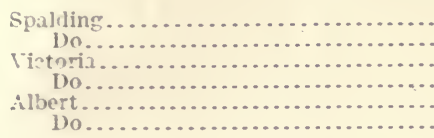 & 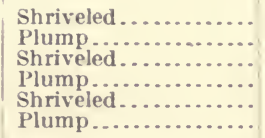 & $\begin{array}{l}2.48 \\
2.33 \\
2.44 \\
2.08 \\
2.59 \\
2.35\end{array}$ \\
\hline
\end{tabular}

Carleton ${ }^{b}$ records the weight of 100 kernels and the percentage of "albuminoids" in sixty-one samples of wheat from various parts of the world. Dividing these into classes according to the weight of 100 kernels we have the following:

\begin{tabular}{|r|r|r|r|}
\hline $\begin{array}{r}\text { Weight of } \\
\text { 100 kernels } \\
\text { (grams). }\end{array}$ & $\begin{array}{c}\text { A rerage } \\
\text { weight of } \\
\text { kernels } \\
\text { (grams). }\end{array}$ & $\begin{array}{c}\text { Percent- } \\
\text { age of albu- } \\
\text { minoids. }\end{array}$ & $\begin{array}{r}\text { Number } \\
\text { of sam- } \\
\text { ples. }\end{array}$ \\
\hline 2 to 3 & 2.66 & 14.58 & 6 \\
3 to 4 & $\begin{array}{l}3.67 \\
\text { over } 4\end{array}$ & 12.31 & 25 \\
\hline
\end{tabular}

Reviewing these experiments there would seem to be no doubt that shrunken kernels contain a higher percentage of nitrogen than do well-filled ones, but as between large and small kernels, both of which are well filled, there is not a great deal of information. Snyder's experiments are the only ones that cover this ground, but they are extensive and very uniform, and may be considered as deciding the question in favor of a higher nitrogen content for the large kernels, so far as small, plump kernels and large, plump kernels are concerned. But, as small and light kernels are usually not plump, taking the crop as a whole and dividing it equally into large and small or heary and light kernels, the evidence would be in favor of the small or light kernels for high nitrogen content. As between wheats from different regions and of different varieties, those having small kernels are generally of higher nitrogen content.

INFLUENCE OF THE SPECIFIC GRAVITY OF THE SEED KERNEL UPON YIELD.

Sanborn ${ }^{c}$ separated seed wheat with a sieve into large, medium, small, and shriveled kernels. The large seed was separated by means

"Handbuch des Getreidebaues, 1, pp. 520-521, Berlin, 1884.

$b$ U. S. Department of Agriculture, Division of Vegetable Physiology and Pathology, Bulletin 24.

c Abstract, Experiment Station Record, 5, p. 58, from Utah Experiment Station Report, 1892 , pp. 133-135. 
of a brine solution into two nearly equal parts. The seed thus separated was planted on separate plots. The experiment was continued three years. The heavy seed yielded 10.8 bushels and the light 16.3 bushels per acre. Unselected seed yielded 16.4 bushels per acre.

Seed wheat of four varieties was separated by Church ${ }^{a}$ by means of solutions of calcium chlorid having specific gravities of 1.247 , 1.293, and 1.31. The seed was first treated with a solution of mercuric chlorid to remove adherent air. Each lot of seed was planted separately. From the results the following conclusions are drawn:

(1) The seed wheat of the greatest density produced the densest seed.

(2) The seed wheat of the greatest density yielded the largest amount of dressed grain.

(3) The seed of medium density generally gave the largest number of ears, but the ears were poorer than those from the densest seed.

(4) Seed of medium density generally produced the largest number of fruiting plants.

(5) The seed wheat that sank in water, but floated in a solution having the density 1.247 , was of very low value, yielding on an average only 34.4 pounds of dressed grain for every 100 yielded by the densest seed.

Haberlandt, ${ }^{b}$ as the result of experiments with several cereals, has shown that the comparative weight of kernels is transmitted to the grain resulting from this seed. This was the case with wheat, rye, barley, and oats. The results with wheat were as follows:

\begin{tabular}{|c|c|c|c|c|}
\hline \multirow{2}{*}{ Number of pounds. } & \multirow{2}{*}{ s } & \multicolumn{3}{|c|}{ Weight of kernels. } \\
\hline & & Light. & Medium. & Heavy. \\
\hline 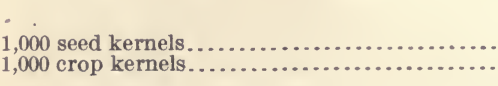 & ... & $\begin{array}{r}\text { Grams. } \\
29.5 \\
34.3\end{array}$ & $\begin{array}{r}\text { Grams. } \\
31.2 \\
35.5\end{array}$ & $\begin{array}{r}\text { Grams. } \\
33.0 \\
36.3\end{array}$ \\
\hline
\end{tabular}

Wollny ${ }^{c}$ objects to the results of the experiments by F. Haberlandt, Church, Trommer, Hellriegel, and $\mathrm{Ph}$. Dietrich with various cereals, in which almost without exception the kernels of high specific gravity produced the best yields, because no distinction was made between absolute weight and specific gravity in the kernels. He claims that the value of the seed lies in the kernels of absolutely heavy weight rather than in the kernels of high specific gravity. He concludes that the specific gravity of the seed exerts no influence on the yield of the crop.

c Abstract in Centrlb. f. Agr. Chem., 1887, p. 169, from Forschungen a.d. Gebiete Agrikulturphysik, 9 . (1886), pp. 207-216. 
In the light of the experiments that have been conducted with seed wheat of high and low specific gravities, it would appear that, in general, seed of very low specific gravity does not yield well, and it is evident that such seed must be deficient in mineral matter and is probably not normal in other respects. There would not appear, however, to be any marked difference in the productive capacity of kernels of medium specific gravity and kernels of great specific gravity.

\section{RELATION OF SPECIFIC GRAVITY OF KERNEL TO NITROGEN CONTENT.}

Marek $^{a}$ found that with an increase in the specific gravity of the kernel there was a decrease in nitrogen content.

Pagnoul, ${ }^{b}$ in testing seventy varieties of wheat, found that the nitrogen content rose with the specific gravity, but not regularly, and that a definite relation could not be traced.

Wollny $^{c}$ took kernels of horny structure and kernels of mealy structure. He says it is generally recognized that the hard, horny kernels have a higher specific gravity, and that it is commonly attributed to their higher content of proteids. He contends that as starch has a higher specific gravity than protein the mealy kernels must really have a higher specific gravity than the horny ones.

Körnicke and Werner ${ }^{l}$ state the specific gravities of the various chemical constituents of the wheat kernel as follows: Starch, 1.53; sugar, 1.60; cellulose, 1.53; fats, 0.91 to 0.96 ; gluten, 1.297 ; ash, 2.50 ; water, 1.00 ; air, 0.001293 . They state also (p. 121) that the specific gravity of the kernel does not stand in any relation to the volume weight, for the factor which results from weighing a certain volume mass is influenced by the air spaces between the kernels, and these depend upon the form and size as well as the surface and accidental structure of the kernel. They also contend that there is no relation between the volume weight and the content of proteid material.

Schindler ${ }^{e}$ shows that by tabulating a large number of varieties of wheat from different parts of the world, and representing different varieties, there is no relation between the weight of 1,000 kernels and the volume weight of 100 c.c. By separating these into varieties,. even when grown in different localities, kernels of the same variety did show a definite and constant relation. The volume weight increased with an increase in the weight of 1,000 kernels.

a Abstract in Centrlb. f. Agr. Chem., 1876, p. 46, from Landw. Zeitung f. Westfalen u. Lippe, 1875 , p. 362.

$b$ Abstract in Centrlb. f. Agr. Chem., 1888, p. 767, from Ann. Agron., 14, pp.-262-272.

c Abstract in Centrlb. f. Agr. Chem., 1887, p. 169, from Forschungen a.d. Gebiete Agrikulturphysik, 9 (1886), pp. 207-216.

"Handbuch des Getreidebaues, 2, p. 120, Berlin, 1884.

$\epsilon$ Jour. Landw., 45 (1897), p. 61. 
There has long. been a desire manifested by workers in this field to establish some definite relation between the specific gravity of the wheat kernel and its composition, or at least its nitrogen content. Very contradictory results have been obtained by several experimenters, and little progress has been made.

It is true that the various chemical constituents that go to compose the wheat kernel have different specific gravities, and as those of the carbohydrates are all less than those of the proteids it might be argued that a wheat having a large proportion of proteid material would have a low specific gravity. However, the specific gravity of the ash is so much greater than that of any other constituent and the ash in wheats from different soils and climates varies so much that these factors completely prevent the establishment of a definite relation. The size and number of the vacuoles also influence the specific gravity.

In general, it may be said that as between kernels of the same variety grown in the same season and upon the same soil, the specific gravity is inversely proportional to the nitregen content.

CONDITIONS AFFECTING THE PRODUCTION OF NITROGEN IN THE GRAIN.

So far as the writer has been able to ascertain there is no literature bearing directly upon the conditions affecting the production of nitrogen in the grain of wheat.

Regarding high nitrogen in the wheat crop as arising merely from failure on the part of the kernel to develop fully, it would seem that a high percentage of nitrogen would inevitably be accompanied by a small production of nitrogen per acre. This, however, does not always appear to be the case.

Taking, for instance, the yields of wheat obtained by Lawes and Gilbert $^{a}$ for a period of twenty years, which they divide into two periods of good and of poor crops, each covering ten years, we have the following figures:

\begin{tabular}{|c|c|c|c|}
\hline Seasons. & $\begin{array}{c}\text { Average } \\
\text { yield of } \\
\text { grain per } \\
\text { acre } \\
\text { (pounds). }\end{array}$ & $\begin{array}{l}\text { Weight } \\
\text { per bushel } \\
\text { (pounds). }\end{array}$ & $\begin{array}{l}\text { Yield of } \\
\text { nitrogen } \\
\text { per acre } \\
\text { (pounds). }\end{array}$ \\
\hline $\begin{array}{l}\text { Good crop seasons... } \\
\text { Poor crop seasons... }\end{array}$ & $\begin{array}{l}1,833 \\
1,740\end{array}$ & $\begin{array}{l}60.2 \\
57.1\end{array}$ & $\begin{array}{l}28.0 \\
29.8\end{array}$ \\
\hline
\end{tabular}

It will be noticed that the largest production of nitrogen per acre was in those years in which the weight per bushel and the yield per acre were least.

Of course this is not always the case, but that it should occur at all is an indication that the conditions that make for high nitrogen

$a$ On the Composition of the Ash of Wheat Grain and Wheat Straw, London, 1884. 
content in the grain also conduce to a large accumulation of nitrogen by the crop, or perhaps it would be more accurate to say that the conditions which favor a large accumulation of nitrogen by the crop often result in giving it a high nitrogen content.

Reference has already been made to the observations of Dehérain and Dupont ${ }^{\text {a }}$ on the wheat crops of 1888 and 1889 at Grignon. The figures for the yields of grain, the percentages of starch and gluten, and the production per acre of these constituents for the two years are as follows:

\begin{tabular}{|c|c|c|c|c|c|}
\hline \multirow{2}{*}{ Year. } & \multirow{2}{*}{$\begin{array}{l}\text { Yield of } \\
\text { grain per } \\
\text { hectare } \\
\text { (kilos). }\end{array}$} & \multicolumn{2}{|c|}{ Percentage of- } & \multirow{2}{*}{$\begin{array}{l}\text { Gluten per } \\
\text { hectare } \\
\text { (kilos). }\end{array}$} & \multirow{2}{*}{$\begin{array}{l}\text { Starch per } \\
\text { hectare } \\
\text { (kilos). }\end{array}$} \\
\hline & & Gluten. & Starch. & & \\
\hline $\begin{array}{l}18 s 5 . \ldots \ldots \ldots \ldots \ldots \\
1859 . \ldots \ldots \ldots \ldots\end{array}$ & $\begin{array}{l}3,445 \\
2,922\end{array}$ & $\begin{array}{l}12.6 \\
15.3\end{array}$ & $\begin{array}{l}77.2 \\
61.9\end{array}$ & $\begin{array}{l}434 \\
447\end{array}$ & $\begin{array}{l}2,659 \\
1,808\end{array}$ \\
\hline
\end{tabular}

From this it will be seen that for the year in which the yield of grain was less per acre the production of gluten per acre was greater. Apparently the conditions were favorable for a large accumulation of nitrogen by the plant in 1889 , but were unfavorable to the production of starch. If the latter had not been the case, the crop of 1889 would have been larger than the crop of 1888 .

A number of instances of this kind have occurred among the wheat crops at the Nebraska Experiment Station. In fact, it may be said that, in general, large yields of grain have there been accompanied by a low percentage of nitrogen per acre as compared with the same properties in small yields of grain. The following table will show this:

Production of nitrogen per acre in wheat raised at the Nebraska Experiment Station.

\begin{tabular}{|c|c|c|c|c|c|}
\hline Variety. & Year. & $\begin{array}{l}\text { Yield of } \\
\text { grain } \\
\text { per acre } \\
\text { (pounds). }\end{array}$ & $\begin{array}{l}\text { Percent- } \\
\text { age of } \\
\text { proteid } \\
\text { nitrogen. }\end{array}$ & $\begin{array}{c}\text { Proteid } \\
\text { nitrogen } \\
\text { per acre } \\
\text { (pounds). }\end{array}$ & $\begin{array}{l}\text { Date of } \\
\text { ripen- } \\
\text { ing. }\end{array}$ \\
\hline \multirow{4}{*}{ 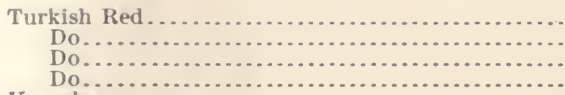 } & 19 & 1,980 & 3.02 & 52. & June 27 \\
\hline & & & & & 24 \\
\hline & & & & & June 23 \\
\hline & & & & & July 9 \\
\hline \multirow{2}{*}{$\begin{array}{r}\text { Yaroslav } \\
\quad \text { Do }\end{array}$} & 19 & & & & July \\
\hline & & & & & July 1 \\
\hline \multirow{2}{*}{$\begin{array}{r}\text { Do } \\
\text { Weissenburg } \\
\text {. }\end{array}$} & & & & & July 14 \\
\hline & 19 & & & & June 24 \\
\hline \multirow{2}{*}{$\begin{array}{r}\text { Pester Boden } \\
\text { Do } \ldots \ldots\end{array}$} & 19 & & & & July 10 \\
\hline & 19 & 1 , & & & \multirow{3}{*}{$\begin{array}{l}\text { June } 24 \\
\text { July } 10\end{array}$} \\
\hline 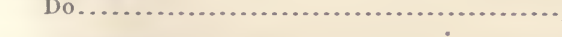 & & & & & \\
\hline & & 1,717 & & 41.43 & \\
\hline
\end{tabular}

a Yield decreased by lodging of grain.

A word in regard to the character of the seasons that produced these crops may help to an understanding of their differences. 
The season of 1900 was rather dry and hot from the time growth started in the spring until harvest. There was no time when there was an abundant supply of moisture, but occasional rains wet the soil for a few days at a time. The temperatures during the day were high and the air was dry. In 1901 the spring was quite moist and cool until June, when it became extremely hot and dry. A few days before harvest the temperatures ranged above $100^{\circ} \mathrm{F}$. daily, with no rainfall. The season of 1902 was the direct opposite of that of 1901, except that the change came earlier. It was extremely dry and hot until the middle of May, when abundant rains came, and the temperatures were considerably below normal until harvest. The season of 1903 was wet and cool throughout.

In general; it may be said that in those seasons, like 1900 and 1902 , in which the temperatures were high and moisture scarce during all or the early part of the growing season, the grain had a high percentage of nitrogen, and there was a large production of nitrogen per acre. In years of low temperatures and abundant moisture, as in 1903, or even when such conditions obtained late in the season, as in 1901, there were a low percentage of nitrogen in the grain and a small production of nitrogen per acre.

High temperatures and scant moisture during early growth would, therefore, seem to favor the accumulation of nitrogen by the wheat plant.

It may also be noted that these are the conditions favorable to the process of nitrification and to the accumulation of nitrates near the surface of the soil.

Comparing the wheat crops grown at Rothamsted for a period of twenty years, the yields and nitrogen production of which have just been stated, with the averages for the Nebraska-grown wheats contained in the last table, it will be seen that the yields of grain were larger at Rothamsted, but that the production of nitrogen per acre was considerably greater in Nebraska. ${ }^{a}$

\begin{tabular}{|c|c|c|}
\hline \multirow{2}{*}{ Station. } & \multicolumn{2}{|c|}{$\begin{array}{l}\text { Yield (in pounds) } \\
\text { per acre of-- }\end{array}$} \\
\hline & Grain. & Nitrogen. \\
\hline 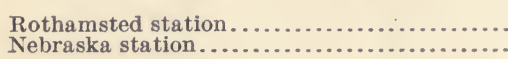 & $\begin{array}{l}1,786 \\
1,717\end{array}$ & $\begin{array}{l}28.9 \\
41.4\end{array}$ \\
\hline
\end{tabular}

The maximum production of nitrogen per acre at Rothamsted during the twenty years was 38.1 pounds, while at Nebraska it was 52.7 pounds.

There can be little doubt as to whether this difference was due in greater measure to soil fertility or to climate. Nowhere is better

$a$ The yield of nitrogen at Rothamsted is calculated from total organic nitrogen, while at the Nebraska Station it is from proteid nitrogen. 
tillage given or are crops more scientifically provided with food than at Rothamsted. It is true that of the ten plots of land on which these wheats were raised one received no manure and three were not sufficiently manured. In order to make the comparison more favorable to the Engfish environment, the five plots completely manured and producing the largest yields may be taken. The yield of nitrogen per acre was 36.4 pounds for the years 1852-1861 and 34.6 pounds for 1862-1871. Even with the best manuring the yields of nitrogen fall very much short of those in Nebraska.

In Nebraska no commercial fertilizers had ever been used on the land on which the wheats were grown, but farm manure had been applied. The soil was a heavy one, well adapted to wheat growing, and had been well tilled. It had been well manured for corn in a rotation of corn, oats, and wheat. The varieties, with the exception of Turkish Red, had just been introduced from Europe and had not fully adapted themselves to the new environment. The average nitrogen production for the only acclimated variety, Turkish Red, was 48 pounds per acre. It would seem, therefore, that a climate affording high temperatures, dry air, and a moderately dry soil is favorable to the accumulation of a large amount of nitrogen by the wheat plant, provided there is a large supply of nitrogen in the soil.

The heat and scant soil moisture are doubtless instrumental in making available the nitrogen of the humus, and the bright sunshine and dry, hot air stimulate growth and increase transpiration.

It has just been said that hot, dry weather in the early growing season contributes to a large nitrogen accumulation by the wheat plant. The same conditions cut short the growing period of the plant and prevent the large accumulation of starch that takes place in the kernel of wheat raised in a cool or moist region. It thus happens that such wheats are high in nitrogen and low in starch.

The properties of the wheat kernel characteristic of a continental climate and rich soil are probably due to rapid nitrification and highly stimulated growth causing a large accumulation of nitrogen by the crop, and to incomplete maturation, caused either by heat, or frost, or lack of moisture, resulting in high nitrogen.

It would be interesting to know what relation the production of nitrogen per acre bears to the production of mineral matter, but the necessary figures are not at hand.

The wheat kernel produced in a continental climate is not usually plump as compared with the kernel produced in an insular or coastal one. The yield of grain per acre is also usually less. That this is due to incomplete maturation is shown by the fact that winter varieties of wheat that make their growth early in the season always yield better than spring varieties. The latter, on the other hand, have a higher percentage of nitrogen, but usually not so large a 
nitrogen production. Their disadvantage lies in the fact that their roots are not sufficiently developed to absorb a large quantity of nitrogenous matter at the time most favorable for its accumulation. As a maximum nitrogen accumulation is the chief desideratum, spring wheats are not desirable where winter ones can be grown.

This does not mean that a variety of wheat which has been grown, for instance, in England will show all the qualities of an inland wheat when first grown in Kansas or Nebraska. Such a wheat will undergo modifications that will give it some of these qualities, such, for instance, as less well-filled kernels, and less weight per bushel. On the other hand, the Turkish Red wheat, when raised in a cool, moist climate, becomes later maturing, and the kernel becomes plumper, more starchy, and softer. It is between varieties adapted each to its peculiar climate, and raised there for years, that these distinctions are most marked, but the fact that a modification of any variety begins at once when transferred from one climate to another shows that such qualities as those mentioned are influenced by the climate.

It must be quite apparent, although it has not often been remarked, that the ordinary selection of seed wheat to increase the yield has resulted in producing a grain of lower nitrogen content.

This has been noticed by Girard and Lindet ${ }^{a}$ and by Biffen, ${ }^{b}$ and incidentally by Balland, ${ }^{c}$ who, in commenting on the decrease in the nitrogen content of wheat in northern France and the increased yields, attributes the former to a deficiency of nitrogen in the fertilizers used, and states that the gluten in the wheat of that region in 1848 ranged from 10.23 to 13.02 per cent, while fifty years later it ranged from 8.96 to 10.62 per cent. In the same time the average yield increased from 14 to 17.5 hectoliters per hectare. In the light of the results of experiments to ascertain the effect of nitrogenous fertilizers upon the composition of wheat, it can not be supposed that this decrease in nitrogen content can be due primarily to lack of nitrogen. It would seem more likely that the increased yield was largely due to the deposition of starch in the grain, and that consequently the percentage of gluten was smaller.

Has the improvement in the yield of wheat been accompanied by a greater yield of nitrogen per acre? It is evident that the increase in the grain and that in the nitrogen are not proportional, but it is

$a$ Le Froment et sa Monture, Paris, 1903.

$b$ Nature (London), 69 (1903), No. 1778, pp. 92, 93.

c Abstract in Centrlb. f. Agr. Chem., 1897, p. 785, from Compt. Rend., 124 (1897), p. 158. 
desirable to know whether there has been any increase in nitrogen per acre. Returning to the figures given by Balland it will be seen that the wheat of 1848 produced on an average 163 kilos per hectare, while that of fifty years later produced 171 kilos, an increase of about 5 per cent in gluten per hectare, with an increase of 25 per cent in yield. These figures can not, of course, be taken as strictly accurate, as they are based merely on what M. Balland refers to as the range of nitrogen content.

Some data on this subject are available in the published records of wheat improvement at the Minnesota Experiment Station. ${ }^{a}$ Yields and gluten content of improved varieties and of the original variety from which the improved strains have been developed by selection are given. The figures cover the same seasons for all varieties, and the averages of six trials are reported for each, as follows:

\begin{tabular}{|c|c|c|c|c|}
\hline Variety. & $\begin{array}{l}\text { Yield fer } \\
\text { acre } \\
\text { (bushels). }\end{array}$ & $\begin{array}{l}\text { Percent- } \\
\text { age of } \\
\text { dry glu- } \\
\text { ten. }\end{array}$ & $\begin{array}{c}\text { Gluten } \\
\text { per acre } \\
\text { (pounds). }\end{array}$ & $\begin{array}{l}\text { Nitrogen } \\
\text { per acre } \\
\text { (pounds). }\end{array}$ \\
\hline $\begin{array}{l}\text { Minnesota No. 149, produced from Power's Fife........... } \\
\text { Power's Fife, unmodified by selection................... } \\
\text { Minnesota No. 1t9, produced from Hayne's Blue Stem.... } \\
\text { Hayne's Blue Stem, unmodified by selection ............. }\end{array}$ & $\begin{array}{l}25.6 \\
23.6 \\
28.5 \\
24.6\end{array}$ & $\begin{array}{l}13.5 \\
14.0 \\
12.5 \\
13.4\end{array}$ & $\begin{array}{l}207.4 \\
198.2 \\
213.7 \\
198.8\end{array}$ & $\begin{array}{l}36.4 \\
34.8 \\
37.5 \\
34.7\end{array}$ \\
\hline
\end{tabular}

In each case the new variety yielded more grain per acre, possessed a lower gluten content, and produced more nitrogen per acre in the grain. It should be explained that determinations of gluten and baking tests were made of strains of wheat produced by the selection of individual plants, and that the quantity and quality of the gluten in these strains were considered in deciding which strain was to be perpetuated. For that reason the gluten content of the improved wheat is doubtless greater than it would have been if no attention had been paid to those qualities. Incidentally it may be stated that the quality of the gluten in these new varieties of wheat originated by Professor Hays is much better than that in the original varieties. The difference between selection for gluten carried on in this way and selection for gluten applied to the individual plant is that the latter must increase many times the opportunity for developing a strain of desirable gluten content.

Returning to the nitrogen production per acre, it is apparent that it is slightly greater in the improved wheats, or at least is not less than in the original varieties. This is encouraging, as it indicates the possibility of increasing the production of gluten per acre.

a Minnesota Experiment Station Bulletin 63. 
Gluten is the valuable constituent of wheat. The wheat growing of the future may be looked upon as a gluten-producing industry. The problem is to secure the highest possible quantity and quality of gluten per acre. If this can be done by sacrificing starch production, it will be economical. Starch can be more cheaply produced in other crops and, if necessary, added to the flour of wheat.

It may be argued that this is not to the interest of the farmer. But it is clearly to the interest of mankind and any step toward its accomplishment must in the end redound to the advantage of the farmer. 
PART II.

EXPERIMENTAL. 



\section{SOME PROPERTIES OF THE WHEAT KERNEL.}

If a number of wheat kernels of the same variety and raised under similar conditions are separated into approximately equal parts with regard to their specific gravity, the kernels of low specific gravity will be found to contain a higher percentage of both total and proteid nitrogen than the kernels having a high specific gravity.

A number of samples of wheat grown in different years and representing different varieties were separated into approximately equal parts by throwing the kernels into a solution of calcium chlorid having such a density that about half the kernels would float and the other half sink. The specific gravity of the solution in which each sample was separated is given in Table 1 and the signs $<$ and $>$ are used to represent "less than" and "greater than," respectively. Thus " $<1.29$ " means that the kernels have a specific gravity of less than 1.29 , while " $>1.29$ " indicates that the kernels have a specific gravity greater than 1.29 .

TABLE 1.-Analyses of kernels of high and of low specific gravity.

\begin{tabular}{|c|c|c|c|c|c|}
\hline \multirow[b]{2}{*}{ Serial number. } & \multirow[b]{2}{*}{$\begin{array}{l}\text { Specific } \\
\text { gravity. }\end{array}$} & \multicolumn{3}{|c|}{ Percentage of- } & \multirow[b]{2}{*}{$\begin{array}{c}\text { Name of variety and year of } \\
\text { growth. }\end{array}$} \\
\hline & & $\begin{array}{c}\text { Total } \\
\text { nitrogen. }\end{array}$ & $\begin{array}{c}\text { Proteid } \\
\text { nitrogen." }\end{array}$ & $\begin{array}{c}\text { Nonpro- } \\
\text { teld } \\
\text { nitrogen. }\end{array}$ & \\
\hline 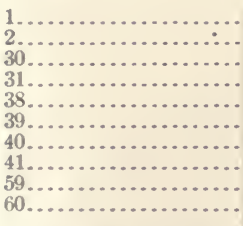 & $\begin{array}{l}<1.290 \\
>1.290 \\
<1.286 \\
>1.286 \\
<1.250 \\
>1.250 \\
<1.265 \\
>1.265 \\
<1.264 \\
>1.264\end{array}$ & $\begin{array}{l}3.51 \\
3.27 \\
2.51 \\
2.51 \\
2.80 \\
2.78 \\
2.95 \\
2.66 \\
3.30 \\
3.06\end{array}$ & $\begin{array}{l}2.49 \\
2.39 \\
1.88 \\
1.94 \\
2.26 \\
2.15 \\
2.13 \\
2.01 \\
2.41 \\
2.29\end{array}$ & $\begin{array}{l}1.02 \\
.88 \\
.63 \\
.57 \\
.54 \\
.63 \\
.82 \\
.65 \\
.89 \\
.77\end{array}$ & $\left\{\begin{array}{l}\text { Hickman, grown in } 1895 . \\
\text { Turkish Red, grown in } 1897 . \\
\text { Spring wheat, Marvel, grown } \\
\text { in } 1897 \text {. } \\
\text { Spring wheat, Velvet Chaff } \\
\text { grown in } 1897 \text {. } \\
\text { Turkish Red, grown in } 1898 .\end{array}\right.$ \\
\hline
\end{tabular}

a Proteid nitrogen in this paper = nitrogen by Stutzer's method. Proteids $=$ proteid nitrogen $\times 5.7$.

With the exception of serial Nos. 30 and 31 the kernels of low specific gravity have in each case a higher percentage of both total and proteid nitrogen than have the kernels of high specific gravity. It will also be noticed that the percentage of nonproteid nitrogen is greater in the kernels of low specific gravity.

Samples of wheat were also divided into light and heavy portions by means of a machine which operates by directing upward a current of air, the velocity of which can be regulated. Into this current the grain is directed. The result is that the heavy kernels and the large 
kernels fall, and the light kernels and small kernels are driven out. The separation thus accomplished is somewhat different from that effected by a solution, the difference being that the latter separates the kernels entirely according to their specific gravities while with the air blast a large kernel of a certain specific gravity might descend with the heavy kernels, when if it were smaller, although of the same specific gravity, it would be blown out.

The number of light kernels that descend on account of their large size is relatively small, owing to the fact that large kernels are, as a rule, of higher specific gravity than small ones. The following test was made to determine the relation between the size of wheat kernels and their specific gravity. An average lot of wheat was nearly equally divided by means of two sieves into three portions representing medium, small, and large kernels. Each of these portions was then thrown upon solutions of the same specific gravity, and the proportion by weight that floated, or light seed, and the proportion that sank, or heavy seed, were determined.

TABLE 2.-Proportion of light and of heavy seed.

\begin{tabular}{|c|c|c|c|c|}
\hline \multirow{2}{*}{ Kind of seed. } & \multirow{2}{*}{$\begin{array}{l}\text { Heavy seed } \\
\text { (grams). }\end{array}$} & \multirow{2}{*}{$\begin{array}{l}\text { Light seed } \\
\text { (grams). }\end{array}$} & \multicolumn{2}{|c|}{ Ratio. } \\
\hline & & & Heavy. & Light. \\
\hline $\begin{array}{l}\text { Small } \\
\text { Medium } \\
\text { Large } .1\end{array}$ & $\begin{array}{r}8.72 \\
9.62 \\
11.96\end{array}$ & $\begin{array}{r}11.28 \\
10.78 \\
8.04\end{array}$ & $\begin{array}{l}1 \\
1 \\
1\end{array}$ & $\begin{array}{r}1.29 \\
1.12 \\
.67\end{array}$ \\
\hline
\end{tabular}

The weight of light kernels among the small was nearly twice that of light kernels among the large seeds.

Analyses of samples of wheat separated by this machine into light and heavy kernels gave about the same results as the samples separated by solutions of certain specific gravities.

TABLE 3.-Analyses of large, heavy kernels and of small, light kernels.

\begin{tabular}{|c|c|c|c|c|c|}
\hline \multirow[b]{2}{*}{ Serial number. } & \multirow[b]{2}{*}{$\begin{array}{l}\text { Relative } \\
\text { weight. }\end{array}$} & \multicolumn{3}{|c|}{ Percentage of- } & \multirow[b]{2}{*}{$\begin{array}{c}\text { Name of variety and year of } \\
\text { growth. }\end{array}$} \\
\hline & & $\begin{array}{c}\text { Total } \\
\text { nitrogen. }\end{array}$ & $\begin{array}{l}\text { Proteid } \\
\text { nitrogen. }\end{array}$ & $\begin{array}{c}\text { Nonpro- } \\
\text { teid } \\
\text { nitrogen. }\end{array}$ & \\
\hline 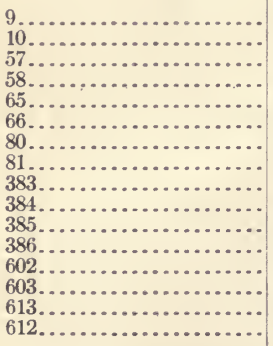 & 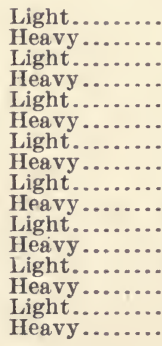 & $\begin{array}{l}2.99 \\
2.76 \\
2.77 \\
2.70 \\
2.91 \\
2.65 \\
2.45 \\
2.19 \\
3.12 \\
3.02 \\
3.13 \\
2.95 \\
3.30 \\
2.46 \\
2.35 \\
2.11\end{array}$ & $\begin{array}{l}2.21 \\
2.04 \\
2.11 \\
2.04 \\
2.29 \\
2.04 \\
2.00 \\
1.96 \\
3.10 \\
2.93 \\
2.82 \\
2.65 \\
3.06 \\
2.24 \\
2.13 \\
1.94\end{array}$ & $\begin{array}{l}0.78 \\
.72 \\
.66 \\
.66 \\
.62 \\
.61 \\
.45 \\
.23 \\
.02 \\
.09 \\
.31 \\
.30 \\
.24 \\
.22 \\
.22 \\
.17\end{array}$ & $\left\{\begin{array}{l}\text { Spring wheat, Marvel, grown } \\
\text { in } 1896 . \\
\text { Currell, grown in } 1898 . \\
\text { Spring wheat, grown in } 1898 . \\
\text { Big Frame, grown in } 1899 . \\
\text { Turkish Red, grown in } 1900 . \\
\text { Big Frame, grown in } 1900 . \\
\text { Big Frame, grown in } 1901 . \\
\text { Turkish Red, grown in } 1901 .\end{array}\right.$ \\
\hline
\end{tabular}


It thus becomes very apparent that the percentage of nitrogen is relatively greater in the light wheat selected in the manner described.

It is well known that inmature wheat is of lighter weight than mature wheat and that it contains a greater percentage of nonproteid nitrogen. In a field of wheat there are always certain plants that mature early, others that mature late, and some that never reach a normal state of maturity. The last condition is very likely to occur in a region of limited rainfall and intense summer heat. The conditions most favorable for the filling out of the grain are shown to be an abundance of soil moisture and a fair degree of warmth. The more nearly the conditions are the reverse of this the more shriveled the kernel and the lighter the weight. In the same variety and in the same field there are kernels that are small and shriveled because of immaturity, disease, or lack of nutriment. All of these classes would appear among the "light" kernels separated in this way.

In order to approach the question from another standpoint, a number of spikes of wheat of the Turkish Red variety were selected in the field, care being taken that all were fully ripe, and that they were composed of healthy, well-formed kernels. These spikes were sampled by removing one row of spikelets from each spike and the kernels so removed were tested for moisture, proteid nitrogen, specific gravity, and weight of kernel, and from the last two the relative volume was calculated. It will be shown later that a sample taken in this way permits of an accurate estimation of the average composition of the kernels on the spike.

The number of grams of proteid nitrogen in the row of spikelets on each spike was calculated from the data mentioned, and the arerage weight of the kernels on the row of spikelets was determined from their total weight and number, thus permitting of the estimation of the number of grams of proteid nitrogen in the average kernel on each spike.

In Table 4 the spikes having a proteid nitrogen content of from 2 to 2.5 per cent are arranged in one group, and on the same line with each spike are placed the number of kernels on one row of spikelets, weight of these kernels, weight of average kernel, relative volume of average kernel, specific gravity of kernel, grams of proteid nitrogen in one row of spikelets, and grams of proteid nitrogen in average kernel. Spikes having a proteid nitrogen content of from 2.5 to 3 per cent are similarly arranged, and so with all spikes up to 4 per cent. The average for each group is shown in the table.

There are, in all, 257 spikes, of which 18 have from 2 to 2.5 per cent proteid nitrogen, 82 from 2.5 to 3 per cent, 107 from 3 to 3.5 per cent, and 49 from 3.5 to 4 per cent. 
TABLE 4.-Analyses of spikes of wheat, arranged according to nitrogen content of kernels. Crop of 1902.

2 TO 2.5 PER CENT PROTEID NITROGEN.

\begin{tabular}{|c|c|c|c|c|c|c|c|c|}
\hline \multirow{2}{*}{$\begin{array}{l}\text { Record } \\
\text { number. }\end{array}$} & \multirow{2}{*}{$\begin{array}{l}\text { Number } \\
\text { of ker- } \\
\text { nels on } \\
\text { row of } \\
\text { spikelets. }\end{array}$} & \multicolumn{2}{|c|}{$\begin{array}{c}\text { Weight (in grams) } \\
\text { of- }\end{array}$} & \multirow{2}{*}{$\begin{array}{c}\text { Volume } \\
\text { of aver- } \\
\text { age ker- } \\
\text { nel. }\end{array}$} & \multirow{2}{*}{$\begin{array}{c}\text { Specific } \\
\text { gravity } \\
\text { of ker-. } \\
\text { nels. }\end{array}$} & \multirow{2}{*}{$\begin{array}{l}\text { Percent- } \\
\text { age of } \\
\text { proteid } \\
\text { nitrogen } \\
\text { in ker- } \\
\text { nels. }\end{array}$} & \multicolumn{2}{|c|}{$\begin{array}{l}\text { Proteid nitrogen } \\
\text { (gram) in- }\end{array}$} \\
\hline & & Kernels. & $\begin{array}{l}\text { Average } \\
\text { kernel. }\end{array}$ & & & & Kernels. & $\begin{array}{l}\text { Average } \\
\text { kernel. }\end{array}$ \\
\hline $\begin{array}{l}183 \ldots \ldots \\
188 \ldots \ldots \\
193 \ldots \ldots \\
205 \ldots \ldots \\
291 \ldots \ldots \\
304 \ldots \ldots \\
318 \ldots \ldots \\
347 \ldots \ldots \\
357 \ldots \ldots \\
358 \ldots \ldots \\
380 \ldots \ldots \\
396 \ldots \ldots \\
402 \ldots \ldots \\
406 \ldots \ldots \\
415 \ldots \ldots \\
440 \ldots \ldots \\
444 \ldots \ldots \\
445 \ldots \ldots\end{array}$ & $\begin{array}{l}17 \\
16 \\
14 \\
15 \\
15 \\
18 \\
21 \\
22 \\
15 \\
15 \\
15 \\
21 \\
14 \\
19 \\
17 \\
21 \\
13 \\
17 \\
16 \\
16\end{array}$ & $\begin{array}{r}0.4772 \\
.4425 \\
.3724 \\
.4824 \\
.5221 \\
.5336 \\
.6708 \\
.4549 \\
.4063 \\
.6689 \\
.4336 \\
.4787 \\
.4594 \\
.5878 \\
.2771 \\
.4566 \\
.4110 \\
.4318\end{array}$ & $\begin{array}{r}0.0280 \\
.0276 \\
.0266 \\
.0321 \\
.0290 \\
.0254 \\
.0304 \\
.0303 \\
.0270 \\
.0318 \\
.0309 \\
.0251 \\
.0258 \\
.0279 \\
.0213 \\
.0268 \\
.0256 \\
.0269\end{array}$ & 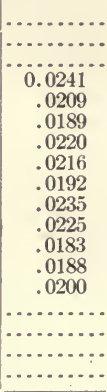 & 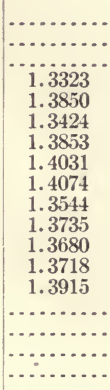 & $\begin{array}{l}2.06 \\
2.37 \\
2.41 \\
2.41 \\
2.23 \\
2.24 \\
2.02 \\
2.44 \\
2.36 \\
2.33 \\
2.35 \\
2.29 \\
2.33 \\
2.44 \\
2.44 \\
2.36 \\
2.38 \\
2.37\end{array}$ & $\begin{array}{r}0.00983 \\
.01049 \\
.00897 \\
.01548 \\
.01616 \\
.01195 \\
.01354 \\
.01110 \\
.00959 \\
.01559 \\
.01019 \\
.01091 \\
.01070 \\
.01434 \\
.00676 \\
.01078 \\
.00978 \\
.01023\end{array}$ & $\begin{array}{r}0.000577 \\
.000654 \\
.000642 \\
.000774 \\
.000647 \\
.000569 \\
.000614 \\
.000739 \\
.000637 \\
.000742 \\
.000726 \\
.000572 \\
.000601 \\
.000681 \\
.000520 \\
.000632 \\
.000609 \\
.000638\end{array}$ \\
\hline Average... & 17 & .4759 & .0266 & .0209 & 1.374 & 2.323 & .01141 & .000643 \\
\hline
\end{tabular}

2.5 TO 3 PER CENT PROTEID NITROGEN.

\begin{tabular}{|c|c|c|c|c|c|c|c|c|}
\hline $181 \ldots \ldots \ldots$ & 19 & 0.4482 & 0.0235 & & & 2.66 & 0.01192 & 0.000625 \\
\hline $182 \ldots \ldots \ldots$ & 17 & .4299 & .0252 & & $\ldots \ldots$ & 2.76 & .01187 & .000696 \\
\hline $185 \ldots . .$. & 19 & .5041 & .0265 & . & & 2.71 & .01366 & .000718 \\
\hline $187 \ldots \ldots$ & 15 & .3945 & .0263 & ...... & & 2.99 & .01180 & .000786 \\
\hline $189 \ldots \ldots$ & 18 & .4871 & .0270 & ........ & 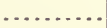 & 2.64 & .01286 & .000713 \\
\hline $196 \ldots \ldots \ldots$ & 17 & .4995 & .0293 & .......... & .......... & 2.71 & .01354 & .000794 \\
\hline $197 \ldots \ldots$ & 20 & .5683 & .0284 & $\ldots \ldots \ldots$ & ........... & 2.85 & .01620 & .000809 \\
\hline $199 \ldots \ldots$ & 17 & .4589 & .0269 & . & . & 2.99 & .01372 & .000804 \\
\hline $207 \ldots \ldots$ & 15 & .4584 & .0305 & 0.0230 & 1. 3248 & 2.73 & .01709 & .000833 \\
\hline $210 \ldots \ldots$ & 14 & .3955 & .0282 & .0288 & 1. 2363 & 2.95 & .01167 & .000832 \\
\hline $211 \ldots .$. & 17 & .5211 & .0306 & .0228 & 1. 3416 & 2.90 & .01511 & .000887 \\
\hline $212 \ldots . .$. & 15 & .4298 & .0286 & .0211 & 1.3537 & 2.97 & .01277 & .000849 \\
\hline $217 \ldots \ldots$ & 18 & .6299 & .0349 & .0259 & 1. 3461 & 2.86 & .01802 & .000998 \\
\hline $218 \ldots \ldots$ & 18 & .5130 & .0285 & .0214 & 1. 3303 & 2.58 & .01324 & .000735 \\
\hline $219 \ldots \ldots$ & 19 & .3862 & .0203 & .0157 & 1.2950 & 2.71 & .01047 & .000550 \\
\hline $222 \ldots$ & 19 & .4611 & .0242 & .0182 & $1.333 \mathrm{i}$ & 2.93 & .01351 & .000709 \\
\hline $227 \ldots$ & 19 & .5581 & .0293 & .0214 & 1.3704 & 2.71 & .01624 & .000794 \\
\hline $229 \ldots \ldots$ & 17 & .4819 & .0285 & .0206 & 1. 3856 & 2.96 & .01387 & .000844 \\
\hline $230 \ldots \ldots$ & 15 & .4867 & .0324 & .0234 & 1.3815 & 2.54 & .01236 & .000823 \\
\hline $238 \ldots \ldots$ & 17 & .5166 & .0303 & .0220 & 1. 3794 & 2.70 & .01395 & $.00 u 818$ \\
\hline $239 \ldots \ldots$ & 17 & .3910 & .0230 & .01649 & 1.3941 & 2.60 & .01017 & .000598 \\
\hline $241 \ldots \ldots$ & 18 & .4230 & .0235 & .0178 & 1.3196 & 2.76 & .01168 & $.000 \mathrm{o} 49$ \\
\hline $242 \ldots \ldots$. & 18 & .4562 & .0253 & .0184 & 1.3753 & 2.96 & .01350 & $.00 \cup 749$ \\
\hline $252 \ldots \ldots$. & 19 & .4898 & .02578 & .0186 & 1.3875 & 2.55 & .01249 & .000055 \\
\hline $277 \ldots \ldots$ & 14 & .3792 & .0270 & .0203 & 1. 3286 & 2.86 & .01085 & $.00 u 772$ \\
\hline $288 \ldots \ldots$ & 17 & .4956 & .0291 & .0217 & 1.3428 & 2.82 & .01398 & .000821 \\
\hline $289 \ldots \ldots$ & 19 & .5042 & .0265 & .0187 & 1.4155 & 2.53 & .01276 & .000670 \\
\hline $293 \ldots$ & 17 & .4858 & .0285 & .0206 & 1.3835 & 2.64 & .01283 & .000752 \\
\hline $294 \ldots \ldots$ & 19 & .4173 & .0219 & .0159 & 1. 3813 & 2.56 & .01068 & .000561 \\
\hline $302 \ldots \ldots$ & 22 & .5569 & .0253 & .0190 & 1. 3312 & 2.68 & .01437 & .000678 \\
\hline $306 \ldots . .$. & 19 & .4922 & .0258 & .0185 & 1. 3996 & 2.51 & .01235 & .000650 \\
\hline $308 \ldots \ldots$. & 15 & .4951 & .0330 & .0237 & 1.392 & 2.85 & .01411 & .000941 \\
\hline $315 \ldots \ldots$ & 16 & .4994 & .0312 & .0224 & 1.3916 & 2.75 & .01373 & .000858 \\
\hline $319 \ldots \ldots \ldots$ & 17 & .4644 & .0273 & .0203 & 1.3447 & 2.86 & .01328 & .000781 \\
\hline $320 \ldots \ldots$. & 18 & .5668 & .0314 & .0229 & 1.3710 & 2.98 & .01689 & .000938 \\
\hline $322 \ldots \ldots \ldots$ & 16 & .5107 & .0219 & .0236 & 1.352 & 2.55 & .01302 & .000813 \\
\hline $329 \ldots \ldots \ldots$ & 12 & .3903 & .0325 & .0234 & 1.3911 & 2.88 & .01241 & .000936 \\
\hline $330 \ldots \ldots$ & 17 & .3431 & .0201 & .0161 & 1.2498 & 2.62 & .00899 & .000527 \\
\hline $332 \ldots \ldots \ldots$ & 16 & .4847 & .0302 & .0218 & 1. 3879 & 2.58 & .01251 & .000779 \\
\hline $334 \ldots$. & 18 & .5399 & .0299 & .0215 & 1. 3922 & 2.62 & .01415 & .000783 \\
\hline $335 \ldots \ldots$ & 18 & .6474 & .0359 & .0258 & 1.3928 & 2.82 & .01826 & .001012 \\
\hline $337 \ldots$ & 15 & .4497 & .0299 & .0215 & 1.3877 & 2.89 & .01345 & .000864 \\
\hline $340 \ldots$ & 20 & .4155 & .0207 & .0153 & 1.3550 & 2.74 & .01138 & .000567 \\
\hline $341 \ldots$ & 15 & .5058 & .0337 & .0243 & 1.3890 & 2.97 & .01502 & .001001 \\
\hline $342 \ldots$ & 14 & .4486 & .0320 & .0228 & 1. 4037 & 2.60 & .01166 & .000832 \\
\hline $343 \ldots$ & 13 & .4112 & .0316 & .0224 & 1.4107 & 2.50 & .01028 & .000791 \\
\hline $344 \ldots$ & 16 & .4004 & .0250 & .0184 & 1. 3611 & 2.93 & .01173 & .000733 \\
\hline $345 \ldots$ & 18 & .5422 & .0301 & .0216 & 1. 3919 & 2.56 & .01388 & .000771 \\
\hline $346 \ldots$ & 19 & .6393 & .0336 & .0242 & 1. 3913 & 2.55 & .01630 & .000857 \\
\hline $348 \ldots \ldots \ldots$ & 18 & .6328 & .0351 & .0262 & 1.3415 & 2.88 & .01822 & .001010 \\
\hline
\end{tabular}


TABLE 4.-Analyses of spikes of wheat, arranged according to nitrogen content of kernels. Crop of 1902-Continued.

2.5 TO 3 PER CENT PROTEID NITROGEN-Continued.

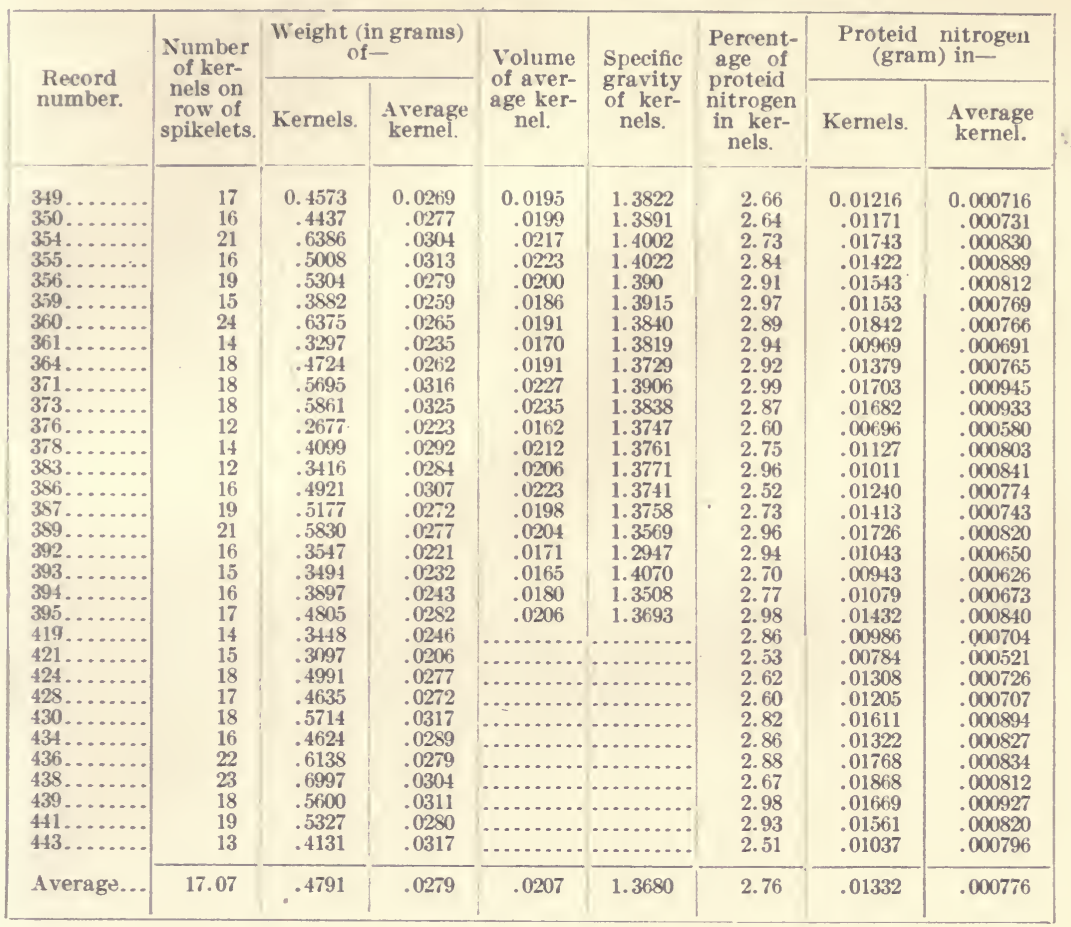

3 TO 3.5 PER CENT PROTEID NITROGEN.

\begin{tabular}{|c|c|c|c|c|c|c|c|c|}
\hline $173 \ldots \ldots$ & 20 & 0.5913 & 0.0295 & & & 3.08 & 0.01821 & 0.000909 \\
\hline $175 \ldots \ldots$. & 21 & .5773 & .0274 & . & - & 3.46 & .01997 & .000948 \\
\hline $176 \ldots \ldots \ldots$ & 20 & .5804 & .0290 & ж. & - & 3.10 & .01799 & .000899 \\
\hline $190 \ldots \ldots \ldots$ & 18 & .4673 & .0259 & ...... & . & 3.25 & .01519 & .000842 \\
\hline $191 \ldots \ldots \ldots$ & 17 & .4279 & .0251 & - & ........ & 3.25 & .01091 & .000816 \\
\hline $192 \ldots \ldots$. & 17 & .4126 & .0242 & (........ & .......... & 3.12 & .01287 & .000755 \\
\hline $194 \ldots . . .$. & 13 & .3218 & .0247 & ......... & $\ldots \ldots \ldots$ & 3.43 & .01104 & .000847 \\
\hline $195 \ldots \ldots \ldots$ & 19 & .4924 & .0259 & ........... & $\ldots \ldots \ldots .$. & 3.33 & .01640 & .000862 \\
\hline $198 \ldots \ldots \ldots$ & 18 & .4683 & .0260 & $\ldots \ldots \ldots$ & .......... & 3.18 & .01489 & .000827 \\
\hline $200 \ldots . . .$. & 18 & .5764 & .0320 & ........... & $\ldots . . . .$. & 3.24 & .01868 & .001040 \\
\hline $202 \ldots . . .$. & 14 & .3824 & .0273 & 0.0200 & 1. 3615 & 3.13 & .01197 & .000854 \\
\hline $203 \ldots . . .$. & 16 & .5251 & .0328 & .0241 & 1.3614 & 3.07 & .01612 & .001007 \\
\hline $206 \ldots . . .$. & 17 & .3392 & .0199 & .0157 & 1.2709 & 3.44 & .01166 & .000685 \\
\hline $208 \ldots . . .$. & 19 & .4939 & .0259 & .0192 & 1. 3494 & 3.21 & .01585 & .000831 \\
\hline $213 \ldots \ldots \ldots$ & 15 & .4116 & .0274 & .0204 & 1.3415 & 3.31 & .01362 & .000907 \\
\hline $214 \ldots . . .$. & 16 & .4371 & .0273 & .0208 & 1. 3082 & 3.09 & .01351 & .000844 \\
\hline $216 \ldots \ldots \ldots$ & 15 & .3122 & .0208 & .0165 & 1. 2588 & 3.33 & .01040 & .000693 \\
\hline $220 \ldots \ldots$. & 17 & .5040 & .0296 & .0222 & 1. 3350 & 3.20 & .01613 & .000947 \\
\hline $223 \ldots \ldots \ldots$ & 17 & .4795 & .0282 & .0204 & 1.3970 & 3.31 & .01587 & .000933 \\
\hline $226 \ldots \ldots \ldots$ & 21 & .5380 & .0256 & .0170 & 1.4951 & 3.11 & .01673 & .000796 \\
\hline $228 \ldots \ldots \ldots$ & 14 & .4143 & .0295 & .0211 & 1.3945 & 3.40 & .01409 & .001003 \\
\hline $231 \ldots \ldots \ldots$ & 18 & .5888 & .0327 & .0242 & 1. 3514 & 3.11 & .01831 & .001017 \\
\hline $232 \ldots \ldots \ldots$ & 13 & .3825 & .0294 & .0221 & 1.3280 & 3.11 & .01190 & .000914 \\
\hline $233 \ldots \ldots \ldots$ & 17 & .5331 & .0313 & .0231 & 1.3558 & 3.32 & .01663 & .001039 \\
\hline $234 \ldots \ldots$. & 16 & .5201 & .0325 & .0243 & 1. 3363 & 3.23 & .01680 & .001050 \\
\hline $236 \ldots \ldots \ldots$ & 25 & .7451 & .0298 & .0220 & 1.3504 & 3. 19 & .02377 & .000951 \\
\hline $243 \ldots \ldots \ldots$ & 24 & .6349 & .0264 & .0196 & 1. 3487 & 3.47 & .02203 & .000916 \\
\hline $244 \ldots \ldots \ldots$ & 19 & .5839 & .0307 & .0214 & 1. 4305 & 3.30 & .01927 & .001013 \\
\hline $249 \ldots \ldots \ldots$ & 16 & .4415 & .0275 & .0199 & 1.3850 & 3.21 & .01417 & .000883 \\
\hline $250 \ldots$ & 15 & .4514 & .0300 & .0213 & 1.4100 & 3. 12 & .01408 & .000936 \\
\hline $251 \ldots$ & 22 & .6190 & .0281 & .0203 & 1.3823 & 3.46 & .02142 & .000972 \\
\hline $255 \ldots$ & 18 & .5948 & .0330 & .0233 & 1.4146 & 3.03 & .01802 & .001000 \\
\hline $256 \ldots$ & 21 & .5277 & .0251 & .0181 & 1. 3629 & 3.31 & .01747 & .000832 \\
\hline $258 \ldots$ & 17 & .4703 & .0276 & .0211 & 1.3065 & 3.38 & .01590 & .000933 \\
\hline
\end{tabular}


TABLE 4.-Analyses of spikes of wheat, arranged according to nitrogen content of kernels. Crop of 1902 - Continued.

3 TO 3.5 PER CENT PROTEID NITROGEN-Continued.

\begin{tabular}{|c|c|c|c|c|c|c|c|c|}
\hline \multirow{2}{*}{$\begin{array}{l}\text { Record } \\
\text { number. }\end{array}$} & \multirow{2}{*}{$\begin{array}{l}\text { Number } \\
\text { of ker- } \\
\text { nels on } \\
\text { row of } \\
\text { spikelets. }\end{array}$} & \multicolumn{2}{|c|}{$\begin{array}{c}\text { Weight (in grams) } \\
\text { of- }\end{array}$} & \multirow{2}{*}{$\begin{array}{c}\text { Volume } \\
\text { of aver- } \\
\text { age ker- } \\
\text { nel. }\end{array}$} & \multirow{2}{*}{$\begin{array}{c}\text { Specific } \\
\text { gravity } \\
\text { of ker- } \\
\text { nels. }\end{array}$} & \multirow{2}{*}{$\begin{array}{l}\text { Percent- } \\
\text { age of } \\
\text { proteid } \\
\text { nitrogen } \\
\text { in ker- } \\
\text { nels. }\end{array}$} & \multicolumn{2}{|c|}{$\begin{array}{l}\text { Proteid nitrogen } \\
\text { (gram) in- }\end{array}$} \\
\hline & & Kernels. & $\begin{array}{l}\text { Average } \\
\text { kernel. }\end{array}$ & & & & Kernels. & $\begin{array}{l}\text { Average } \\
\text { kernel. }\end{array}$ \\
\hline $262 \ldots \ldots . .$. & 18 & 0.4604 & 0.0255 & 0.0193 & 1.3216 & 3.20 & 0.01473 & 0.000816 \\
\hline $263 \ldots \ldots \ldots$ & 18 & .5040 & .0280 & .0197 & 1.4206 & 3.24 & .01633 & .000907 \\
\hline $264 \ldots . . .$. & 18 & .4138 & .0229 & .0169 & 1.3544 & 3.37 & .01395 & .000772 \\
\hline $265 \ldots . . .$. & 18 & .4429 & .0246 & .0189 & 1.3005 & 3.30 & .01462 & .000812 \\
\hline $266 \ldots \ldots \ldots$ & 19 & .5010 & .0263 & .0187 & 1.4090 & 3.11 & .01558 & .000818 \\
\hline $269 \ldots \ldots$ & 17 & .4531 & .0266 & .0209 & 1.2748 & 3.21 & .01454 & .000854 \\
\hline $270 \ldots \ldots$ & 20 & .5183 & .0259 & .0191 & 1. 3541 & 3.37 & .01747 & .000873 \\
\hline $271 \ldots . .$. & 14 & .3275 & .0233 & .0177 & 1.3143 & 3.39 & .01110 & .000790 \\
\hline $272 \ldots . .$. & 15 & .3858 & .0257 & .0190 & 1. 3564 & $3: 14$ & .01212 & .000807 \\
\hline $273 \ldots . .$. & 18 & .4559 & .0253 & .0178 & 1.4228 & 3.39 & .01546 & .000858 \\
\hline $275 \ldots . . .$. & 18 & .4862 & .0270 & .0197 & 1.3711 & 3.33 & .01619 & .000899 \\
\hline $276 \ldots \ldots$. & 15 & .3973 & .0264 & .0191 & 1.3815 & 3.15 & .01251 & .000832 \\
\hline $278 \ldots \ldots \ldots$ & 15 & .4715 & .0314 & .0226 & 1.3903 & 3.12 & .01471 & .000980 \\
\hline $281 \ldots . . .$. & 21 & .6938 & .0330 & .0241 & 1.3693 & 3.26 & .02262 & .001076 \\
\hline $282 \ldots . .$. & 18 & .4973 & .0276 & .0200 & 1.3795 & 3.02 & .01502 & .000834 \\
\hline $295 \ldots \ldots$ & 19 & .5205 & .0273 & .0201 & 1.3608 & 3.06 & .01593 & .000835 \\
\hline $300 \ldots \ldots$ & 19 & .4994 & .0262 & .0188 & 1.3945 & 3.07 & .01533 & .000894 \\
\hline $301 \ldots .$. & 16 & .5492 & .0343 & .0249 & 1. 3787 & 3.09 & .01697 & .001060 \\
\hline $305 \ldots \ldots$ & 13 & .3452 & .0265 & .0197 & 1. 3432 & 3.07 & .01060 & .000814 \\
\hline $307 \ldots \ldots$. & 20 & .4122 & .0206 & .0140 & 1.4727 & 3.19 & .01315 & .000657 \\
\hline $310 \ldots . . .$. & 18 & .4867 & .0270 & .0198 & 1. 3681 & 3.16 & .01538 & .000853 \\
\hline $312 \ldots \ldots \ldots$ & 15 & .4324 & .0288 & .0210 & 1.3718 & 3.49 & .01509 & .001005 \\
\hline $314 \ldots \ldots$. & 15 & .4122 & .0274 & .0201 & 1.3657 & 3.16 & .01303 & .000866 \\
\hline $316 \ldots \ldots$. & 17 & .4157 & .0244 & .0178 & 1. 3733 & 3.36 & .01397 & .000820 \\
\hline $317 \ldots \ldots$. & 17 & .4412 & .0259 & .0193 & 1.3424 & 3.43 & .01513 & .000888 \\
\hline $321 \ldots \ldots$. & 18 & .5484 & .0304 & .0207 & 1. 4660 & 3.43 & .01881 & .001043 \\
\hline $323 \ldots \ldots \ldots$ & 17. & .4075 & .0239 & .0177 & 1.3487 & 3.43 & .01398 & .000820 \\
\hline $324 \ldots \ldots \ldots$ & 17 & .4230 & .0248 & .0180 & 1.3740 & 3.19 & .01349 & .000791 \\
\hline $325 \ldots \ldots \ldots$ & 17 & .5110 & .0300 & .0220 & 1. 3658 & 3.46 & .01768 & .001038 \\
\hline $327 \ldots \ldots \ldots$ & 16 & .4039 & .0252 & .0191 & 1.3225 & 3.45 & .01393 & .000869 \\
\hline $333 \ldots \ldots \ldots$ & 16 & .4610 & .0288 & .0206 & 1.3956 & 3.26 & .01503 & .000939 \\
\hline $336 \ldots \ldots \ldots$ & 13 & .3637 & .0279 & .0198 & $1.41 \mathrm{n} 2$ & 3.36 & .01222 & .000937 \\
\hline $339 \ldots \ldots \ldots$ & 16 & .3803 & .0237 & .0171 & 1.3828 & 3.33 & .01266 & .000789 \\
\hline $351 \therefore . . .$. & 15 & .3843 & .0256 & .0186 & 1.3812 & 3.32 & .01276 & .000851 \\
\hline $352 \ldots \ldots \ldots$ & 15 & .4497 & .0299 & .0217 & $1.38 \%$ & 3.05 & .01372 & .000914 \\
\hline $353 \ldots \ldots$. & 16 & .4726 & .0295 & .0211 & 1. 3988 & 3.11 & .01470 & .000917 \\
\hline $362 \ldots \ldots \ldots$ & 19 & .5258 & .0276 & .0201 & $1.3^{7} 01$ & 3.03 & .01593 & .000836 \\
\hline $366 \ldots \ldots \ldots$ & 17 & .4214 & .0247 & .0185 & 1.3350 & 3.17 & .01336 & .000783 \\
\hline $367 \ldots \ldots \ldots$ & 20 & .5351 & .0267 & .0197 & 1.3555 & 3.37 & .01803 & .000900 \\
\hline $368 \ldots \ldots \ldots$ & 19 & .3877 & .0204 & .0151 & 1. 3497 & 3.06 & .01186 & .000624 \\
\hline $369 \ldots \ldots \ldots$ & 19 & .5560 & .0292 & .0214 & 1. 3621 & 3.34 & .01857 & .000975 \\
\hline $370 \ldots \ldots$ & 17 & .4200 & .0247 & .0180 & 1.3735 & 3.09 & .01298 & .000763 \\
\hline $372 \ldots \ldots$ & 17 & .4811 & .0283 & .0206 & 1. 3714 & 3.31 & .01593 & .000937 \\
\hline $374 \ldots \ldots$. & 17 & .5249 & .0308 & .0218 & 1.4142 & 3.15 & .01653 & .000970 \\
\hline $375 \ldots \ldots$ & 18 & .5147 & .0285 & .0203 & 1.4018 & 3.41 & .01755 & .000975 \\
\hline $377 \ldots \ldots$. & 14 & .3173 & .0226 & .0174 & 1. 3013 & 3.47 & .01101 & .000784 \\
\hline $379 \ldots \ldots$ & 18 & .5271 & .0292 & .0213 & , 1.3703 & 3.09 & .01629 & .000902 \\
\hline $381 \ldots \ldots$ & 13 & .3506 & .0269 & .0199 & 1.3544 & 3.45 & .01210 & .000928 \\
\hline $382 \ldots \ldots \ldots$ & 19 & .5057 & .0266 & .0194 & 1.3728 & 3.23 & .01633 & .000859 \\
\hline $388 \ldots \ldots \ldots$ & 19 & .5799 & .0305 & .0221 & 1. 3773 & 3.05 & .01769 & .000930 \\
\hline $390 \ldots \ldots$. & 19 & .4764 & .0250 & .0181 & 1.3806 & 3.22 & .01534 & .000805 \\
\hline $391 \ldots \ldots$ & 18 & .4474 & .0248 & .0182 & 1. 3628 & 3.26 & .01459 & .000808 \\
\hline $399 \ldots \ldots$ & 12 & .3058 & .0254 & .0188 & 1. 3510 & 3.10 & .00948 & .000787 \\
\hline $400 \ldots \ldots$ & 20 & .5720 & .0286 & .0206 & 1. 3837 & 3.35 & .01916 & .000958 \\
\hline $401 \ldots$. & 16 & .3996 & .0249 & .0183 & 1. 3575 & 3.37 & .01347 & .000839 \\
\hline $403 \ldots \ldots$ & 17 & .5000 & .0294 & .0211 & 1. 3927 & 3.04 & .01520 & .000894 \\
\hline $404 \ldots . .$. & 18 & .4286 & .0238 & .0180 & 1.3221 & 3.30 & .01414 & .000785 \\
\hline $410 \ldots \ldots$ & 20 & .5368 & .0268 & ....... & $\ldots . . . . .$. & 3.27 & .01755 & .000780 \\
\hline $411 \ldots \ldots \ldots$ & 14 & .3479 & .0248 & $\ldots . .$. & ........... & 3.15 & .01096 & .000781 \\
\hline $414 \ldots . . .$. & 19 & .5044 & .0265 & $\ldots \ldots$ & ........... & 3.14 & .01584 & .000832 \\
\hline $416 \ldots . .$. & 15 & .4269 & .0284 & ....... & ........... & 3.24 & .01383 & .000920 \\
\hline $418 \ldots \ldots \ldots$ & 21 & .4995 & .0237 & $\ldots \ldots \ldots$ & ........... & 3.05 & .01523 & .000723 \\
\hline $423 \ldots \ldots . .$. & 18 & .4845 & .0269 & .......... & .......... & 3.14 & .01521 & .000845 \\
\hline $425 \ldots \ldots \ldots$ & 16 & .4801 & .0300 & $\ldots \ldots \ldots$ & .......... & 3.30 & .01584 & .000990 \\
\hline $426 \ldots \ldots \ldots$ & 18 & .5166 & .0287 & ............. & ............ & 3.09 & .01596 & .000887 \\
\hline $427 \ldots \ldots \ldots$ & 19 & .5433 & .0285 & $\ldots \ldots \ldots$ & .......... & 3.06 & .01662 & .000872 \\
\hline $429 \ldots \ldots \ldots$ & 20 & .4704 & .0235 & $\ldots \ldots \ldots$ & ........... & 3.04 & .01430 & .000714 \\
\hline $431 \ldots . .$. & 18 & .4119 & .0228 & ............ & $\ldots \ldots \ldots$ & 3.20 & .01318 & .000732 \\
\hline $432 \ldots \ldots . .$. & 21 & .6306 & .0300 & ............ & ........... & 3.00 & .01892 & .000900 \\
\hline $433 \ldots$ & 20 & .5206 & .0260 & ............. & .............. & 3.12 & .01624 & .000811 \\
\hline $437 \ldots \ldots \ldots$ & 16 & .4336 & .0271 & ........... & ........... & 3.13 . & .03357 & .000848 \\
\hline $442 \ldots$ & 17 & .3889 & .0228 & $\ldots \ldots \ldots$ & $\ldots \ldots \ldots$ & 3.23 & .01256 & .000736 \\
\hline Average .. & 17.4 & .4724 & .0270 & .0199 & 1. 3666 & 3.23 & .01520 & .000874 \\
\hline
\end{tabular}


TABLE 4.-Analyses of spikes of wheat, arranged according to nitrogen content of kernels Crop of 1902-Continued.

3.5 TO 4 PER CENT PROTEID NITROGEN.

\begin{tabular}{|c|c|c|c|c|c|c|c|c|}
\hline \multirow{2}{*}{$\begin{array}{l}\text { Record } \\
\text { number. }\end{array}$} & \multirow{2}{*}{$\begin{array}{l}\text { Number } \\
\text { of ker- } \\
\text { nels on } \\
\text { row of } \\
\text { spikelets. }\end{array}$} & \multicolumn{2}{|c|}{$\begin{array}{c}\text { Weight (in grams) } \\
\text { of - }\end{array}$} & \multirow{2}{*}{$\begin{array}{l}\text { Volume } \\
\text { of aver- } \\
\text { age ker- } \\
\text { nel. }\end{array}$} & \multirow{2}{*}{$\begin{array}{c}\text { Specific } \\
\text { gravity } \\
\text { of ker- } \\
\text { nels. }\end{array}$} & \multirow{2}{*}{$\begin{array}{c}\text { Percent- } \\
\text { age of } \\
\text { proteid } \\
\text { nitrogen } \\
\text { in ker- } \\
\text { nels. }\end{array}$} & \multicolumn{2}{|c|}{$\begin{array}{l}\text { Proteid nitrogen } \\
\text { (gram) in- }\end{array}$} \\
\hline & & Kernels. & $\begin{array}{l}\text { Average } \\
\text { kernel. }\end{array}$ & & & & Kernels. & $\begin{array}{l}\text { Average } \\
\text { kernel. }\end{array}$ \\
\hline $174 \ldots \ldots \ldots$ & 18 & 0.4025 & 0.0223 & & in & 3.76 & 0.01513 & 0.000838 \\
\hline $177 \ldots$ & 19 & .4073 & .0214 & & $\ldots$ & 3.57 & .01454 & .000764 \\
\hline $179 \ldots \ldots$ & 19 & .4972 & .0261 & $\ldots$. & $\ldots$ & 3.85 & .01914 & .001005 \\
\hline $180 \ldots \ldots$ & 17 & .5262 & .0309 & $\ldots$. & $\ldots$ & 3.58 & .01884 & .001110 \\
\hline $184 \ldots . .$. & 20 & .5512 & .0275 & $\ldots$. & $\cdots$ & 3.78 & .02084 & .001040 \\
\hline $186 \ldots . .$. & 21 & .5414 & .0257 & $\ldots$ & & 3.97 & .02149 & .001020 \\
\hline $204 \ldots . .$. & 15 & .4015 & .0267 & 0.0198 & 1.3460 & 3.90 & -.01566 & .001043 \\
\hline $209 \ldots \ldots \ldots$ & 17 & .3588 & .0211 & .0164 & 1. 2828 & 3.82 & .01371 & .000806 \\
\hline $215 \ldots \ldots$ & 12 & .3318 & .0276 & .0205 & 1. 3493 & 3. 79 & .01258 & .001046 \\
\hline $224 \ldots \ldots$ & 17 & .4891 & .0287 & .0220 & 1. 3039 & 3.65 & .01785 & .001048 \\
\hline $225 \ldots \ldots$ & 19 & .4976 & .0261 & .0193 & 1. 3507 & 3.55 & .01766 & .000927 \\
\hline $235 \ldots \ldots \ldots$ & 18 & .4555 & .0253 & .0192 & 1. 3164 & 3.65 & .01663 & .000923 \\
\hline $240 \ldots \ldots \ldots$ & 16 & .3984 & .0249 & .0177 & 1.4025 & 3.53 & .01406 & .000879 \\
\hline $245 \ldots$ & 15 & .3971 & .0264 & .0200 & 1.3230 & 3.64 & .01445 & .000361 \\
\hline $246 \ldots \ldots$ & 18 & .4562 & .0253 & .0194 & 1.3058 & 3.75 & .01711 & .000949 \\
\hline $247 \ldots$ & 18 & .4937 & .0274 & .0202 & 1.3561 & 3.50 & .01728 & .000959 \\
\hline $248 \ldots$ & 17 & .4617 & .0271 & .0193 & 1.4095 & 3.65 & .01685 & .000991 \\
\hline $253 \ldots$ & 21 & .5960 & .0283 & .0203 & 1.3917 & 3.63 & .02163 & .001327 \\
\hline $259 \ldots$ & 19 & .4932 & .0259 & .0193 & 1.3400 & 3.84 & .01894 & .000995 \\
\hline $261 \ldots$ & 17 & .5195 & .0305 & .0229 & 1. 3333 & 3.50 & .01818 & .001068 \\
\hline $274 \ldots$ & 15 & .3347 & .0223 & .0168 & 1.3300 & 3.57 & .01195 & .000796 \\
\hline $279 \ldots \ldots \ldots$ & 16 & .4304 & .0269 & .0200 & 1.3441 & 3.79 & .01631 & .001020 \\
\hline $280 \ldots \ldots . .$. & 16 & .4305 & .0269 & .0198 & 1.3600 & 3.70 & .01593 & .000995 \\
\hline $283 \ldots \ldots \ldots$ & 17 & .4974 & .0292 & .0210 & 1.3911 & 3.86 & .01920 & .001127 \\
\hline $284 \ldots \ldots \ldots$ & 14 & .3723 & .0265 & .0189 & 1. 4050 & 3.72 & .01385 & .000986 \\
\hline $285 \ldots \ldots \ldots$ & 18 & .5769 & .0320 & .0233 & 1.3715 & 3.87 & .02233 & .001238 \\
\hline $286 \ldots . . .$. & 17 & .4140 。 & .0243 & .0178 & 1. 3660 & 3.56 & .01474 & .000865 \\
\hline $287 \ldots \ldots$. & 16 & .4740 & .0296 & .0223 & 1. 3270 & 3.87 & .01835 & .001146 \\
\hline $290 \ldots \ldots$ & 16 & .3955 & .0247 & .0177 & 1. 3921 & 4.00 & .01582 & .000988 \\
\hline $296 \ldots \ldots$. & 17 & .5037 & .0296 & .0214 & 1. 3832 & 3.94 & .01985 & .001166 \\
\hline $299 \ldots \ldots \ldots$ & 17 & .4553 & .0267 & .0195 & 1.3715 & 3.68 & .01676 & .000983 \\
\hline $309 \ldots \ldots$ & 18 & .4753 & .0239 & .0239 & 1. 1051 & 3.75 & .01782 & .000990 \\
\hline $313 \ldots \ldots$ & 17 & .4798 & .0282 & .0202 & 1. 3971 & 3.52 & .01689 & .000993 \\
\hline $328 \ldots \ldots$. & 20 & .5795 & .0289 & .0215 & 1. 3466 & 3.61 & .02092 & .001043 \\
\hline $363 \ldots \ldots$. & 17 & .3795 & .0223 & .0165 & 1.3499 & 3.50 & .01328 & .000781 \\
\hline $365 \ldots \ldots \ldots$ & 16 & .3469 & .0216 & .0169 & 1.2787 & 3.50 & .01214 & .000756 \\
\hline $354 \ldots \ldots$ & 14 & .4012 & .0286 & .0212 & 1.3499 & 3.56 & .01428 & .001020 \\
\hline $355 \ldots \ldots$ & 15 & .4162 & .0277 & .0203 & 1.3670 & 3. 79 & .01578 & .001050 \\
\hline $405 \ldots$ & 18 & .4940 & .0274 & .0203 & 1. 3508 & 3. & .01857 & .001030 \\
\hline $407 \ldots \ldots$ & 20 & .4707 & .0235 & .0171 & 1.3700 & 3. & .01784 & .000891 \\
\hline $408 \ldots . .$. & 19 & .4462 & .0234 & ..... & .......... & 3. & .01624 & .000852 \\
\hline $409 \ldots . .$. & 17 & .4329 & .0254 & 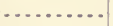 & ........... & 3.59 & .01554 & .000912 \\
\hline$\ldots$. & 16 & .3390 & .0211 & 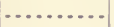 & .......... & 3.63 & .01231 & .000766 \\
\hline $413 \ldots . .$. & 17 & .4393 & .0258 & 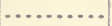 & $\ldots \ldots \ldots$ & 3.77 & .01656 & .000973 \\
\hline $417 \ldots \ldots$ & 19 & .4530 & .0238 & . & .......... & 3.80 & .01721 & .000904 \\
\hline $420 \ldots . . .$. & 17 & .4156 & .0244 & $\ldots$ & .......... & 3.73 & .01550 & .000910 \\
\hline & 23 & .5395 & .0234 & & ............. & 3.53 . & .01904 & .000826 \\
\hline $435 \ldots \ldots \ldots$ & 20 & .4310 & .0215 & & $\ldots$ & 3.53 & .01521 & .000759 \\
\hline $446 \ldots \ldots \ldots$ & 17 & .4425 & .0260 & & $\ldots$ & 3.75 & .01659 & .000975 \\
\hline A verage... & 17.3 & .4517 & .0257 & .01987 & 1.3494 & 3.70 & .01672 & .000982 \\
\hline
\end{tabular}

Table 5 shows at a glance the averages for each of the classes of spikes just tabulated, and permits of a comparison of the average figures for each class. ${ }^{a}$

a The determinations of specific gravity were made by the following method, devised by Prof. S. Avery: A light basket of wire gauze was suspended by a hair from the hook that supported one of the pan hangers of the balance. The basket was allowed to hang in a beaker of benzol supported by a shelf above the pan. By using a counterpoise the balance was now brought to the zero point. The balance was kept at zero by the occasional adjustment of a rider on the left arm of the beam. The wheat was weighed on the pan of the balance, then transferred to the basket and weighed in benzol, and the temperature of the latter carefully noted. The specific gravity was calculated from the well-known formula:

$\frac{\text { Wt. in air } \times \text { sp. gr. in benzol at } \mathrm{T}^{\circ}}{\text { Wt. in air }- \text { wt. in benzol }}==\mathrm{Sp}$. gr. 
TABLE 5.-Summary of analyses of spikes of wheat, arranged according to nitrogen content of kernels. Crop of 1902.

\begin{tabular}{|c|c|c|c|c|c|c|c|c|c|}
\hline \multirow{2}{*}{$\begin{array}{l}\text { Range cf } \\
\text { percentage of } \\
\text { proteid } \\
\text { nitrogen. }\end{array}$} & \multirow{2}{*}{$\begin{array}{c}\text { Per- } \\
\text { centage } \\
\text { of pro- } \\
\text { teid } \\
\text { nitro- } \\
\text { gen in } \\
\text { kernels. }\end{array}$} & \multicolumn{2}{|c|}{ Number of- } & \multicolumn{2}{|c|}{$\begin{array}{c}\text { Weight (in grams) } \\
\text { of- }\end{array}$} & \multirow{2}{*}{$\begin{array}{l}\text { Volume } \\
\text { of aver- } \\
\text { age ker- } \\
\text { nel. }\end{array}$} & \multirow[b]{2}{*}{$\begin{array}{l}\text { Specific } \\
\text { gravity. }\end{array}$} & \multicolumn{2}{|c|}{$\begin{array}{l}\text { Proteid nitrogen } \\
\text { (gram) in- }\end{array}$} \\
\hline & & $\begin{array}{l}\text { Analy- } \\
\text { ses. }\end{array}$ & $\begin{array}{c}\text { Kernels } \\
\text { on row } \\
\text { of spike- } \\
\text { lets. }\end{array}$ & Kernels. & $\begin{array}{l}\text { Average } \\
\text { kernel. }\end{array}$ & & & Kernels. & $\begin{array}{l}\text { Average } \\
\text { kernel. }\end{array}$ \\
\hline $\begin{array}{l}2 \text { to } 2.5 \ldots \ldots \\
2.5 \text { to } 3 \ldots \ldots \\
3 \text { to } 3.5 . \ldots \ldots \\
3.5 \text { to } 4 \ldots \ldots\end{array}$ & $\begin{array}{l}2.32 \\
2.76 \\
3.23 \\
3.70\end{array}$ & $\begin{array}{r}18 \\
82 \\
107 \\
49\end{array}$ & $\begin{array}{l}17 \\
17.1 \\
17.4 \\
17.3\end{array}$ & $\begin{array}{r}0.4759 \\
.4791 \\
.4724 \\
.4715\end{array}$ & $\begin{array}{r}0.0266 \\
.0279 \\
.0270 \\
.0257\end{array}$ & $\begin{array}{r}0.0209 \\
.0207 \\
.0199 \\
.0199\end{array}$ & $\begin{array}{l}1.374 \\
1.368 \\
1.367 \\
1.349\end{array}$ & $\begin{array}{r}0.01141 \\
.01332 \\
.01520 \\
.01672\end{array}$ & $\begin{array}{r}0.000643 \\
.000776 \\
.000874 \\
.000982\end{array}$ \\
\hline
\end{tabular}

From this table it will be seen that with an increase in the percentage of proteid nitrogen the number of kernels on a row of spikelets remains about constant; that in general there were a decrease in the weight of the kernels on a row of spikelets and a slight decrease in the weight of the average kernel; and that the volume of the average kernel decreased, as did the specific gravity.

It may safely be stated that a high percentage of proteid nitrogen was in these spikes associated with a kernel of low specific gravity, light weight, and small relative volume, and, as the spikes were selected for their ripeness and healthy appearance, this relation can not be attributed to immaturity or disease. •

The table last referred to shows a decrease in the weight of the kernels on the spike as the percentage of proteid nitrogen increases; but it also shows that in spite of the decrease in the weight of the kernels there is an increase in the actual amount of proteid nitrogen they contain, and that the same is true of the average kernel.

Table 6 gives a summary of the same analyses, arranged according to the specific gravities of the kernels. All spikes whose kernels had a specific gravity below 1.30 are grouped in one class, those having a specific gravity of 1.30 to 1.33 in another class, and so on until finally all spikes having a specific gravity of more than 1.42 form the last class.

TABLE 6.-Summary of analyses of spikes of wheat, arranged according to specific gravities of kernels. Crop of 1902 .

\begin{tabular}{|c|c|c|c|c|c|c|c|c|}
\hline \multirow{2}{*}{$\begin{array}{l}\text { Range of specific } \\
\text { gravity. }\end{array}$} & \multirow{2}{*}{$\begin{array}{c}\text { Specific } \\
\text { gravity } \\
\text { of ker- } \\
\text { nels. }\end{array}$} & \multicolumn{2}{|c|}{ Number of- } & \multirow{2}{*}{$\begin{array}{c}\text { Weight } \\
\text { of kernels } \\
\text { (gram). }\end{array}$} & \multirow{2}{*}{$\begin{array}{l}\text { Percent- } \\
\text { age of } \\
\text { proteid } \\
\text { nitrogen } \\
\text { in ker- } \\
\text { nels. }\end{array}$} & \multirow{2}{*}{$\begin{array}{c}\text { Weight } \\
\text { of aver- } \\
\text { age } \\
\text { kernel } \\
\text { (gram). }\end{array}$} & \multicolumn{2}{|c|}{$\begin{array}{l}\text { Proteid nitrogen } \\
\text { (gram) in- }\end{array}$} \\
\hline & & $\begin{array}{l}\text { Analy- } \\
\text { ses. }\end{array}$ & Kernels. & & & & Kernels. & $\begin{array}{l}\text { Average } \\
\text { kernel. }\end{array}$ \\
\hline $\begin{array}{l}\text { Below } 1.30 \ldots \ldots \\
1.30 \text { to } 1.33 \ldots \\
1.33 \text { to } 1.36 \ldots \ldots \\
1.36 \text { to } 1.39 \ldots \ldots \\
1.39 \text { to } 1.42 \ldots \ldots \\
1.42 \text { and over } \ldots\end{array}$ & $\begin{array}{l}1.255 \\
1.315 \\
1.347 \\
1.375 \\
1.399 \\
1.463\end{array}$ & $\begin{array}{r}8 \\
17 \\
50 \\
71 \\
40 \\
8\end{array}$ & $\begin{array}{l}16.7 \\
16.5 \\
17.3 \\
17.2 \\
16.7 \\
19.1\end{array}$ & $\begin{array}{r}0.3887 \\
.4315 \\
.4008 \\
.4794 \\
.4848 \\
.5287\end{array}$ & $\begin{array}{l}3.29 \\
3.35 \\
2.91 \\
3.06 \\
3.03 \\
3.07\end{array}$ & $\begin{array}{r}0.02331 \\
.02617 \\
.02366 \\
.02786 \\
.02899 \\
.02773\end{array}$ & $\begin{array}{l}0.01280 \\
.01446 \\
.01508 \\
.01462 \\
.01459 \\
.01605\end{array}$ & $\begin{array}{r}0.0007662 \\
.0008762 \\
.0008756 \\
.0008559 \\
.0008729 \\
.0008371\end{array}$ \\
\hline
\end{tabular}


This table shows no constant relation between the specific gravity and the number of kernels on the spike. With an increase in the specific gravity there is an increase in the weight of the kernels on the spike, and with some exceptions an increase in the weight of the average kernel. As the specific gravity increases, the percentage of proteid nitrogen decreases, which agrees with the previous table. The grams of proteid nitrogen in the kernels on the spikes and in the average kernel increase with the specific gravity.

Table 7 shows the summary of the same analyses, arranged according to the weight of the average kernel. Spikes whose kernels have an average weight of less than 0.024 gram form the first class, and each succeeding class increases by 0.002 gram.

TABLE 7.-Summary of analyses of spikes of wheat, arranged according to weight of average kernel. Crop of 1902.

\begin{tabular}{|c|c|c|c|c|c|c|c|c|}
\hline \multirow{2}{*}{$\begin{array}{l}\text { Range of weight of } \\
\text { average kernel } \\
\text { (gram). }\end{array}$} & \multirow{2}{*}{$\begin{array}{l}\text { Weight } \\
\text { of a rer- } \\
\text { age ker- } \\
\text { nel } \\
\text { (gram). }\end{array}$} & \multicolumn{2}{|c|}{ Number of- } & \multirow{2}{*}{$\begin{array}{l}\text { Weight } \\
\text { of ker- } \\
\text { nels } \\
\text { (gram). }\end{array}$} & \multirow{2}{*}{$\begin{array}{c}\text { Specific } \\
\text { gravity } \\
\text { of ker- } \\
\text { nels. }\end{array}$} & \multirow{2}{*}{$\begin{array}{l}\text { Percent- } \\
\text { age of } \\
\text { proteid } \\
\text { nitrogen } \\
\text { in ker- } \\
\text { nels. }\end{array}$} & \multicolumn{2}{|c|}{$\begin{array}{l}\text { Proteid nitrogen } \\
\quad(\text { gram) in- }\end{array}$} \\
\hline & & $\begin{array}{l}\text { Analy- } \\
\text { ses. }\end{array}$ & Kernels. & & & & $\begin{array}{l}\text { Average } \\
\text { kernel. }\end{array}$ & Kernels. \\
\hline $\begin{array}{l}\text { Below } 0.024 \\
.024 \text { to } 0.026 \\
.026 \text { to } 0.028 \\
.028 \text { to } 0.030 \\
.030 \text { to } 0.032 . \\
.032 \text { and over }\end{array}$ & $\begin{array}{l}0.02214 \\
.02528 \\
.02705 \\
.02896 \\
.03089 \\
.03324\end{array}$ & $\begin{array}{l}27 \\
38 \\
48 \\
40 \\
26 \\
19\end{array}$ & $\begin{array}{l}16.9 \\
17.5 \\
17.0 \\
17.0 \\
17.0 \\
16.8\end{array}$ & $\begin{array}{r}0.3812 \\
.4425 \\
.4609 \\
.4916 \\
.5274 \\
.5588\end{array}$ & $\begin{array}{r}1.341 \\
1.361 \\
1.360 \\
1.372 \\
1.388 \\
1.373\end{array}$ & $\begin{array}{l}3.197 \\
3.28 \\
3.22 \\
3.11 \\
2.86 \\
2.88\end{array}$ & $\begin{array}{r}0.0007184 \\
.0008294 \\
.0008711 \\
.0009090 \\
.0008787 \\
.0009594\end{array}$ & $\begin{array}{r}0.01215 \\
.01438 \\
.01475 \\
.01546 \\
.01506 \\
.01617\end{array}$ \\
\hline
\end{tabular}

There seems to be no relation between the weight of the average kernel and the number of kernels on the spike. The weight of all the kernels on the spike naturally increases with the weight of the average kernel. The specific gravity of the kernels increases with the weight of the average kernel. The percentage of proteid nitrogen decreases with an increase in the weight of the average kernel, in which respect it agrees with the two previous tables. The grams of proteid nitrogen in the average kernel and the total proteid nitrogen in the spike increase with the weight of the average kernel.

Samples from each of the spikes of wheat from which these data were derived were planted, together with samples from other spikes, all of which have been analyzed, aggregating 800 in all. Each kernel was planted separately at a distance of 6 inches each way from every other kernel. The kernels from each spike were marked by a stake bearing the record number of the spike.

During the winter a considerable number of plants were killed, so that the stand was irregular in the spring. In some cases all of the plants resulting from a spike of the previous year were killed, and in other cases only a portion of such plants. The result was a somewhat uneven stand, which doubtless gave certain plants an advantage over others in growth and yield. 
When the crop was ripe in 1903 each plant was harvested separately, and all of those resulting from spikes which the previous year had shown a proteid nitrogen content of more than 4 per cent or less than 2 per cent were analyzed, as were also a certain number resulting from spikes of intermediate values.

The good kernels on each plant were counted and weighed, thus giving a record of the yield of each plant. From these data the average weight of the kernels per plant was calculated. The specific gravity was not determined and consequently the average volume of the kernels on each plant was not calculated, as was done the previous year.

In Table 8 the plants harvested in 1903 are arranged in classes of 1 to 2 per cent proteid nitrogen, 2 to 2.5 per cent, 2.5 to 3 per cent, 3 to 3.5 per cent, 3.5 to 4 per cent, 4 to 4.5 per cent, and over 4.5 per cent. The number and weight of the kernels on each plant are stated, as is also the average weight of each kernel. The number of grams of proteid nitrogen in all the kernels of the plant is shown, and also the number of grams of proteid nitrogen in the average kernel on each plant. Table 9 shows the average for each class.

These results, so far as they cover the same ground as those of the previous year, have the same significance. They show a quite uniform although slight decrease in the weight of the average kernel accompanying an increase in the percentage of proteid nitrogen, and a very marked increase in the number of grams of proteid nitrogen in the average kernel. Especially marked is the increase in the amount of proteid nitrogen in the average kernel, amounting to 28 per cent of the weight of the kernel for every 1 per cent increase in the content of proteid nitrogen.

One column of this table, not contained in that compiled from results of the previous year, shows the number of grams of proteid nitrogen contained in all of the kernels on the plant; or, in other words, the proteid nitrogen production of the plant. This appears, on the whole, to increase with the percentage of proteid nitrogen, although the results are not sufficiently consistent to permit of an unqualified statement to that effect. The uneven stand of the plants; before referred to, doubtless accounts for these inconsistent results.

Two other columns contain data not obtained in 1902. The first of these shows the number of kernels per plant, which apparently decreases slightly as the percentage of proteid nitrogen increases, but this can not be stated unqualifiedly. The next column shows the weight of kernels per plant, or the yield per plant, which likewise seems to decrease slightly. with an increase in the percentage of proteid nitrogen. 
TABLE 8.-Analyses of plants, arranged according to percentage of proteid nitrogen. Crop of. 1903.

1 TO 2 PER CENT PROTEID NITROGEN.

\begin{tabular}{|c|c|c|c|c|c|c|}
\hline \multirow{2}{*}{$\begin{array}{c}\text { Record num- } \\
\text { ber. }\end{array}$} & \multirow{2}{*}{$\begin{array}{l}\text { Percent- } \\
\text { age of } \\
\text { proteid } \\
\text { nitrogen } \\
\text { in kernels. }\end{array}$} & \multirow{2}{*}{$\begin{array}{l}\text { Number } \\
\text { of ker- } \\
\text { nels per } \\
\text { plant. }\end{array}$} & \multicolumn{2}{|c|}{ Weight (in grams) of- } & \multirow{2}{*}{$\begin{array}{l}\text { Total pro- } \\
\text { teid nitro- } \\
\text { gen in all } \\
\text { kernels } \\
\text { (gram). }\end{array}$} & \multirow{2}{*}{$\begin{array}{l}\text { Proteid } \\
\text { nitrogen in } \\
\text { average ker- } \\
\text { nel } \\
\text { (gram). }\end{array}$} \\
\hline & & & $\begin{array}{c}\text { Kernels } \\
\text { per plant. }\end{array}$ & $\begin{array}{c}\text { Average } \\
\text { kernel. }\end{array}$ & & \\
\hline 32206 . & 1.81 & 507 & 10.4036 & 0.02052 & 0.18831 & 0.0003714 \\
\hline $32605 \ldots$ & 1.20 & 225 & 5.2268 & .02323 & .06272 & .0002788 \\
\hline $33407 \ldots$ & 1.62 & 305 & 7.0889 & .02271 & .11223 & .0003679 \\
\hline $33108 \ldots$ & 1.39 & 77 & 1.1132 & .01446 & .01547 & .0002009 \\
\hline $33905 \ldots$ & 1.61 & 508 & 11.1476 & .02194 & .17948 & .0003533 \\
\hline $42206 \ldots$ & 1.46 & 25 & .3161 & .01264 & .00462 & .0001816 \\
\hline $45606 \ldots$ & 1.91 & 220 & 4.0358 & .01834 & .07708 & .0003504 \\
\hline $45805 \ldots$ & 1.81 & 124 & 1.5298 & .01234 & .02815 & .0002700 \\
\hline $48107 \ldots$ & 1.50 & 718 & 11.2890 & .01572 & .16933 & .0002358 \\
\hline $51005 \ldots$ & 1.34 & 862 & 15.5935 & .01804 & .20881 & .0002422 \\
\hline $55307 \ldots$ & 1.89 & 342 & 5.6864 & .01663 & .10747 & .0003142 \\
\hline $57308 \ldots \ldots$ & 1.69 & $5 \pi 7$ & 9.8378 & .01705 & .16626 & .0002881 \\
\hline $57405 \ldots \ldots$ & 1.98 & 41 & .8328 & .02031 & .01649 & .0004022 \\
\hline $57607 \ldots \ldots$ & 1.73 & 736 & 16.4433 & .02234 & .24847 & .0003865 \\
\hline $58 \$ 06 \ldots \ldots \ldots$ & 1.88 & 95 & 1.9469 & .02049 & .03660 & .0003853 \\
\hline $60605 \ldots \ldots$ & 1.87 & 35 & .5952 & .01701 & .01113 & .0003180 \\
\hline $63505 \ldots \ldots$ & 1.90 & 208 & 4.0230 & .01934 & .07644 & .0003674 \\
\hline $69806 \ldots . .$. & 1.66 & 558 & 12.0136 & .02153 & .19943 & .0003574 \\
\hline $72606 \ldots \ldots$ & 1.89 & 543 & 9.3629 & .01724 & .18538 & .0003414 \\
\hline $74305 \ldots \ldots$ & 1.98 & 216 & 4.4222 & .02047 & .08756 & .0004054 \\
\hline $80305 \ldots \ldots$ & 1.81 & 729 & 15.7835 & .02165 & .28569 & .0003919 \\
\hline $81705 \ldots$ & 1.98 & 46.5 & 9.7922 & .02106 & .19388 & .0004170 \\
\hline $81710 \ldots \ldots$ & 1.92 & 396 & 9.1411 & .02308 & .17550 & .0004432 \\
\hline $92407 \ldots \ldots$ & 1.66 & 53 & .8983 & .01695 & .01491 & .0002814 \\
\hline $94205 \ldots . .$. & 1.65 . & 64 & 1.2117 & .01893 & .01999 & .0003124 \\
\hline $94605 \ldots$ & 1.95 & 56 & .7319 & .01307 & .01427 & .0002549 \\
\hline $94908 \ldots$ & 1.96 & 125 & 2.3678 & .01894 & .04641 & .0003713 \\
\hline $95510 \ldots$ & 1.81 & 159 & 2.8356 & .01783 & .05132 & .0003228 \\
\hline Average... & 1. 749 & 320.3 & 6.23823 & .01871 & .10655 & .00032914 \\
\hline
\end{tabular}

2 TO 2.5 PER CENT PROTEID NITROGEN

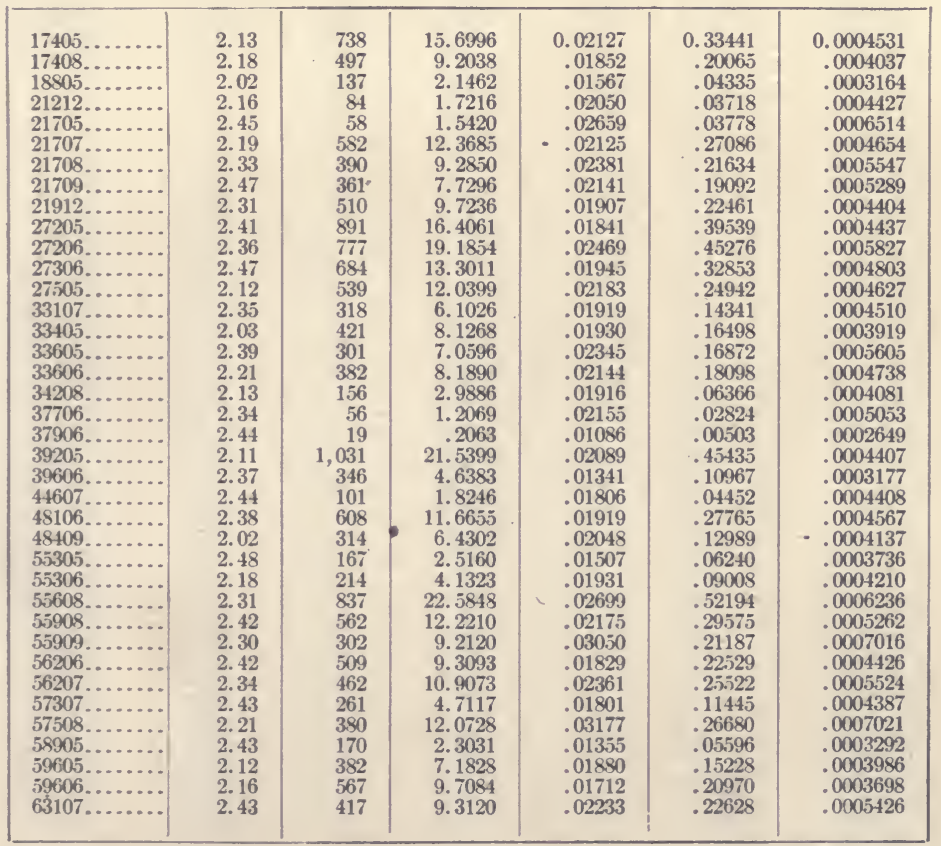


TABLE 8.-Analyses of plants, arranged according to percentage of proteid nitrogen. Crop of 1903-Continued.

2 TO 2.5 PER CENT PROTEID NITROGEN-Continued.

\begin{tabular}{|c|c|c|c|c|c|c|}
\hline \multirow{2}{*}{$\begin{array}{l}\text { Record num- } \\
\text { ber. }\end{array}$} & \multirow{2}{*}{$\begin{array}{l}\text { Percent- } \\
\text { age of } \\
\text { proteid } \\
\text { nitrogen } \\
\text { in kernels. }\end{array}$} & \multirow{2}{*}{$\begin{array}{l}\text { Number } \\
\text { of ker- } \\
\text { nels per } \\
\text { plant. }\end{array}$} & \multicolumn{2}{|c|}{ Weight (in grams) of- } & \multirow{2}{*}{$\begin{array}{l}\text { Total pro- } \\
\text { teid nitro- } \\
\text { gen in all } \\
\text { kernels } \\
\text { (gram). }\end{array}$} & \multirow{2}{*}{$\begin{array}{c}\text { Proteid } \\
\text { nitrogen in } \\
\text { average ker } \\
\text { nel } \\
\text { (gram). }\end{array}$} \\
\hline & & & $\begin{array}{c}\text { Kernels } \\
\text { per plant. }\end{array}$ & $\begin{array}{l}\text { Average } \\
\text { kernel. }\end{array}$ & & \\
\hline $63506 \ldots \ldots$. & 2.44 & 153 & 2.3986 & .01568 & 0.05853 & 0.0003825 \\
\hline $65306 \ldots \ldots$. & 2.41 & 544 & 9.8298 & .01807 & .23690 & .0004355 \\
\hline $65307 \ldots$ & 2.28 & 373 & 7.0051 & .01878 & .15971 & .0004282 \\
\hline 65308 & 2.09 & 583 & 11. 7066 & .02008 & .24468 & .0004197 \\
\hline $69505 \ldots$ & 2.29 & 225 & 4. 7116 & .01847 & .10790 & .0004231 \\
\hline $71905 \ldots$ & 2.47 & 1,260 & 28.2136 & .02239 & .69688 & .0005531 \\
\hline $72705 \ldots$ & 2.13 & 372 & 9.1522 & .02191 & .19936 & .0004668 \\
\hline $72708 \ldots \ldots$ & 2.27 & 398 & 9.0386 & .02270 & .20518 & .0005154 \\
\hline $72905 \ldots \ldots$ & 2.48 & 167 & 2.6462 & .01585 & .06563 & .0003930 \\
\hline $73306 \ldots \ldots$. & 2.45 & 414 & 8.5373 & .02062 & .20918 & .0005052 \\
\hline $73307 \ldots \ldots$ & 2.39 & 25 & .5572 & .02229 & .01332 & .0005327 \\
\hline $74606 \ldots$ & 2.30 & 464 & 9.6451 & .02079 & .22184 & .0004781 \\
\hline $76205 \ldots \ldots$ & 2.35 & 498 & 8.4407 & .01695 & .19836 & .0003983 \\
\hline $81707 \ldots \ldots$ & 2.34 & 786 & 18.3614 & .02336 & .42965 & .0005466 \\
\hline $81708 \ldots \ldots$ & 2.41 & 287 & 7.3993 & .02578 & .17833 & .0006213 \\
\hline $81709 \ldots \ldots$ & 2.28 & 757 & 16.4692 & .02175 & .37548 & .0004960 \\
\hline $84405 \ldots \ldots \ldots$ & 2.48 & 428 & 8.7448 & .02043 & .21687 & .0005067 \\
\hline $84905 \ldots \ldots$ & 2.32 & 37 & .7130 & .01927 & .01654 & .0004471 \\
\hline $88608 \ldots \ldots$ & 2.47 & 74 & 1.5355 & .02075 & .03793 & .0005125 \\
\hline $88609 \ldots \ldots$ & 2.42 & 470 & 9.8719 & .02100 & .23890 & .0005082 \\
\hline $92409 \ldots \ldots$ & 2.30 & 315 & 5.7131 & .01814 & .13140 & .0004171 \\
\hline $94209 \ldots \ldots$ & 2.49 & 190 & 3.6006 & .01895 & .08965 & .0004719 \\
\hline $94406 \ldots$ & 2.47 & 549 & 10.5556 & .01923 & .26073 & .0004749 \\
\hline $94407 \ldots \ldots$ & 2.07 & 419 & 6.7664 & .01615 & .14007 & .0003343 \\
\hline $94905 \ldots \ldots$ & 2.35 & 286 & 4.4423 & .01553 & .10439 & .0003650 \\
\hline $95509 \ldots \ldots$ & 2.48 & 138 & 2.9475 & .02136 & .07310 & .0005297 \\
\hline $95707 \ldots \ldots$ & 2.47 & 52 & .7577 & .01457 & .01872 & .0003599 \\
\hline Average.... & 2.319 & 396.8 & 8.2502 & .020113 & .190316 & .0004660 \\
\hline
\end{tabular}

2.5 TO 3 PER CENT PROTEID NITROGEN.

\begin{tabular}{|c|c|c|c|c|c|c|}
\hline $17409 \ldots \ldots$ & 2.75 & 802 & 14.8957 & 0.01857 & 0.40964 & 0.0005108 \\
\hline $17410 \ldots \ldots$ & 2.88 & 744 & 16.9987 & .02285 & .48957 & .0006580 \\
\hline $20706 \ldots \ldots$ & 2.78 & 163 & 3.3138 & .02033 & .09212 & .0005652 \\
\hline $20707 \ldots \ldots$ & 2.77 & 444 & 9.9070 & .02282 & .27443 & .0006181 \\
\hline $20708 \ldots . .$. & 2.58 & 122 & 2.4690 & .02024 & .06399 & .0005221 \\
\hline $20710 \ldots \ldots$ & 2.83 & 867 & 17.1115 & .01974 & .48428 & .0005586 \\
\hline $21207 \ldots \ldots$ & 2.96 & 118 & 2.3066 & .01955 & .06804 & .0005766 \\
\hline $21305 \ldots \ldots$ & 2.67 & 312 & 6.2514 & .02004 & .16691 & .0005350 \\
\hline $21306 \ldots \ldots$ & 2.90 & 226 & 4. 1516 & .01837 & .12039 & .0005327 \\
\hline $21710 \ldots \ldots$ & 2.59 & 59 & .8478 & .01437 & .02196 & .0003722 \\
\hline $21711 \ldots \ldots$ & 2.71 & 873 & 17.1820 & .01968 & .46563 & .0005334 \\
\hline $21805 \ldots \ldots$ & 2.69 & 1,232 & 20.9290 & .01699 & .56299 & .0004569 \\
\hline $21806 \ldots \ldots$ & 2.71 & 599 & 14.2450 & .02378 & .38604 & .0006444 \\
\hline $21807 \ldots \ldots$ & 2.73 & 377 & 9.4172 & .02498 & .25709 & .0006664 \\
\hline $21808 \ldots \ldots$ & 2.57 & 1,156 & 19.7446 & .01708 & .50744 & .0004389 \\
\hline $21809 \ldots \ldots$ & 2.73 & 418 & 8.0214 & .01919 & .21898 & .0005238 \\
\hline $21810 \ldots \ldots$ & 2.69 & 52 & 1.0304 & .01982 & .02772 & .0005330 \\
\hline $21905 \ldots \ldots$ & 2.64 & 791 & 14. 3111 & .01809 & .37781 & .0004777 \\
\hline $22205 \ldots \ldots$ & 2.81 & 283 & 2. 6965 & .00953 & .07577 & .0002677 \\
\hline $22207 \ldots \ldots$ & 2.77 & 169 & 3. 2787 & .01940 & .09082 & .0005374 \\
\hline $25205 \ldots \ldots$ & 2.71 & 522 & 10.7836 & .02066 & .28560 & .0005599 \\
\hline $25206 \ldots \ldots$ & 2.76 & 205 & 4. 6754 & .02281 & .12904 & .0006295 \\
\hline $26106 \ldots \ldots \ldots$ & 2.63 & 90 & 2.0737 & .02304 & .05454 & .0006060 \\
\hline $26805 \ldots \ldots$ & 2.81 & 220 & 4.9456 & .02248 & .13897 & .0006317 \\
\hline $26806 \ldots . .$. & 2.60 & 152 & 2. 7255 & .01793 & .07086 & .0004662 \\
\hline $26807 \ldots \ldots$ & 2.80 & 721 & 17.2324 & .02390 & .48250 & .0006692 \\
\hline $26905 \ldots \ldots$ & 2.76 & 326 & 6.4102 & .01966 & .17692 & .0005427 \\
\hline $26906 \ldots \ldots$ & 2.71 & 228 & 4.2376 & .01859 & .11484 & .0005037 \\
\hline $26907 \ldots \ldots$ & 2.61 & 102 & 1.8276 & .01792 & .04995 & .0004677 \\
\hline $26908 \ldots \ldots$ & 2.96 & 192 & 3.9797 & .02073 & .11780 & .0006135 \\
\hline $26909 \ldots \ldots$ & 2.80 & 180 & 2.9999 & .01667 & .08400 & .0004667 \\
\hline $27005 \ldots \ldots$ & 2.63 & 866 & 16.4120 & .01895 & .43164 & .0004984 \\
\hline $27207 \ldots \ldots \ldots$ & 2.92 & 166 & 3.3266 & .02004 & .09712 & .0005850 \\
\hline $27305 \ldots \ldots \ldots$ & 2.58 & 267 & 5.5666 & .02085 & .14362 & .0005379 \\
\hline $27307 \ldots \ldots$ & 2.53 & 167 & 3.0850 & .01847 & .07805 & .0004674 \\
\hline $27506 \ldots \ldots$ & 2.70 & 444 & 10.0005 & .02252 & .27003 & .0006082 \\
\hline $27508 \ldots \ldots$ & 2.64 & 251 & 5.5324 & .02287 & .14608 & .0006037 \\
\hline $27009 \ldots \ldots$ & 2.90 & 243 & 5. 3615 & .02206 & .15549 & .0006399 \\
\hline $28805 \ldots \ldots$ & 2.91 & 87 & 2. 1851 & .02512 & .06359 & .0007309 \\
\hline $32606 \ldots \ldots \ldots$ & 2.88 & 94 & 2.0162 & .02145 & .05807 & .0006177 \\
\hline
\end{tabular}


TABLE 8.-Analyses of plants, arranged according to percentage of proteid nitrogen. Crop of 1903 -Continued.

2.5 TO 3 PER CENT PROTEID NITROGEN-Continued.

\begin{tabular}{|c|c|c|c|c|c|c|}
\hline \multirow{2}{*}{$\begin{array}{l}\text { Record num- } \\
\text { ber. }\end{array}$} & \multirow{2}{*}{$\begin{array}{l}\text { Percent- } \\
\text { age of } \\
\text { proteid } \\
\text { nitrogen } \\
\text { in kernels. }\end{array}$} & \multirow{2}{*}{$\begin{array}{l}\text { Number } \\
\text { of ker- } \\
\text { nels per } \\
\text { plant. }\end{array}$} & \multicolumn{2}{|c|}{ Weight (in grams) of- } & \multirow{2}{*}{$\begin{array}{l}\text { Total pro- } \\
\text { teid nitro- } \\
\text { gen in all } \\
\text { kernels } \\
\text { (gram). }\end{array}$} & \multirow{2}{*}{$\begin{array}{l}\text { Proteid } \\
\text { nitrogen in } \\
\text { average ker- } \\
\text { nel } \\
\text { (gram). }\end{array}$} \\
\hline & & & $\begin{array}{l}\text { Kernels } \\
\text { per plant. }\end{array}$ & $\begin{array}{l}\text { Average } \\
\text { kernel. }\end{array}$ & & \\
\hline $33105 \ldots \ldots \ldots$ & 2.91 & 132 & 2.5601 & 0.01939 & 0.07450 & 0.0005644 \\
\hline $33106 \ldots \ldots \ldots$ & 2.94 & 18 & .3089 & .01716 & .00908 & .0005045 \\
\hline $33406 \ldots \ldots$ & 2.87 & 283 & 4.6045 & .01627 & .13215 & .0004670 \\
\hline $33906 \ldots \ldots$ & 2.81 & 119 & 2.2862 & .01921 & .06424 & .0005399 \\
\hline $34205 \ldots \ldots$ & 2.73 & 464 & 9.1498 & .01972 & .24979 & .0005383 \\
\hline $34207 \ldots \ldots$ & 2.81 & 611 & 13.5556 & .02219 & .38505 & .0006273 \\
\hline $37305 \ldots \ldots$ & 2.96 & 309 & 6. 1394 & .01987 & .18173 & .0005881 \\
\hline $37705 \ldots \ldots$ & 2.64 & 461 & 8.0905 & .01972 & .23998 & .0005327 \\
\hline $37707 \ldots \ldots$ & 2.94 & 193 & 3.3004 & .01710 & .09670 & .0005010 \\
\hline $37905 \ldots \ldots \ldots$ & 2.53 & 37 & .9452 & .02555 & .02391 & .0006463 \\
\hline $38005 \ldots \ldots \ldots$ & 2.84 & $\begin{array}{r}37 \\
139\end{array}$ & 2.5134 & .01808 & .07138 & .0005135 \\
\hline $38506 \ldots \ldots \ldots$ & 2.89 & 85 & 1.6799 & .01975 & .04855 & .0005712 \\
\hline $386,06 \ldots \ldots$ & 2.63 & 401 & 8.4605 & .02110 & .22251 & .0005549 \\
\hline $38608 \ldots \ldots \ldots$ & 2.82 & 158 & 3.0228 & .01913 & .08522 & .0005394 \\
\hline $38609 \ldots \ldots$ & 2.74 & 293 & 6.7665 & .02309 & .18540 & .0006475 \\
\hline $38706 \ldots \ldots$ & 2.59 & 365 & 7.2545 & .01988 & .18789 & .0005148 \\
\hline $39405 \ldots \ldots$. & 2.88 & 447 & 9.3541 & .02093 & .21399 & .0006027 \\
\hline $39506 \ldots . .$. & 2.93 & 67 & 1.9218 & .02869 & .05631 & .0008404 \\
\hline $40505 \ldots \ldots$ & 2.82 & 170 & 4.1546 & .02444 & .11716 & .0006892 \\
\hline $43405 \ldots$ & 2.92 & 124 & 2.8000 & .02258 & .08176 & .0006594 \\
\hline $44505 \ldots \ldots$ & 2.94 & 340 & 5.9990 & .01764 & .17637 & .0005187 \\
\hline $44605 \ldots .$. & 2.86 & 55 & 1. 1271 & .02049 & .03223 & .0005861 \\
\hline $44606 \ldots \ldots$ & 2.90 & 124 & 2.5235 & .02035 & .07318 & .0005902 \\
\hline $45605 \ldots \ldots$ & 2.82 & 61 & .7081 & .01161 & .01997 & .0003273 \\
\hline $46106 \ldots \ldots$ & 2.54 & 82 & 1.6103 & .01964 & .04090 & .0004988 \\
\hline $46107 \ldots \ldots$ & 2.54 & 478 & 8.3935 & .01756 & .21319 & .0004460 \\
\hline $48305 \ldots \ldots$ & 2.87 & 473 & 12.0278 & .02543 & .34524 & .0007299 \\
\hline $48408 \ldots \ldots$ & 2.81 & 27 & .3485 & .01291 & .00979 & .0003627 \\
\hline $48507 \ldots \ldots$ & 2.64 & 70 & 1.6036 & .02296 & .04233 & $.000 \approx 062$ \\
\hline $48508 \ldots \ldots$ & 2.76 & 603 & 11.2008 & .01858 & .30986 & .0005127 \\
\hline $48806 . .$. & 2.70 & 547 & 9.8346 & .01798 & .26553 & .0004877 \\
\hline $50706 \ldots . .$. & 2.80 & 35 & .4701 & .01343 & .01316 & .0003761 \\
\hline $55008 \ldots \ldots \ldots$ & 2.60 & 944 & 17.4226 & .01846 & .45299 & .0004799 \\
\hline $55206 \ldots \ldots$ & 2.56 & 578 & 11. 3592 & .01965 & .29079 & .0005031 \\
\hline $55308 \ldots \ldots \ldots$ & 2.54 & 397 & 9.5078 & .02395 & .24150 & .0006225 \\
\hline $55506 \ldots \ldots$ & 2.80 & 866 & 17.8506 & .02062 & .49995 & .0005773 \\
\hline $55507 \ldots \ldots \ldots$ & 2.63 & 504 & 9.8228 & .01949 & .25834 & .0005126 \\
\hline $55605 \ldots \ldots$ & 2.64 & 500 & 10.9180 & .02184 & .28823 & .0005765 \\
\hline $55606 \ldots \ldots$ & 2.58 & 503 & 11.0930 & .02205 & .28580 & .0005690 \\
\hline $55607 \ldots \ldots$ & 2.69 & 138 & 2.3931 & .01734 & .06437 & .0004665 \\
\hline $55905 \ldots \ldots \ldots$ & 2.67 & 331 & 5.7948 & .01751 & .15170 & .0004674 \\
\hline $55906 \ldots \ldots$ & 2.81 & 499 & 7.9968 & .01603 & .22471 & .0004503 \\
\hline $55907 \ldots \ldots \ldots$ & 2.59 & 749 & 19.3966 & .02590 & .50238 & .0006707 \\
\hline $56105 \ldots \ldots \ldots$ & 2.73 & 336 & 5. 7431 & .01709 & .15679 & .0004667 \\
\hline $56106 \ldots \ldots$. & 2.57 & 644 & 12.0161 & .01866 & .30881 & .0004795 \\
\hline $56107 \ldots \ldots$ & 2.96 & 872 & 14.4556 & .01658 & .42790 & .0004907 \\
\hline $56205 \ldots \ldots \ldots$ & 2.51 & 333 & 6.5232 & .01959 & .16373 & .0004917 \\
\hline $56208 \ldots \ldots$ & 2.61 & 563 & 13.5720 & .02356 & .34616 & .0006149 \\
\hline $56209 \ldots \ldots$ & 2.59 & 950 & 15.8086 & .01664 & .40945 & .0004310 \\
\hline $57005 \ldots . .$. & 2.71 & 88 & 1.5364 & .01746 & .04164 & .0004731 \\
\hline $57006 \ldots \ldots$ & 2.76 & 701 & 10.1836 & .01453 & .28107 & .0004010 \\
\hline $57007 \ldots \ldots$ & 2.65 & 168 & 3.3176 & .01975 & .08792 & .0005233 \\
\hline $57105 \ldots \ldots$ & 2.76 & 407 & 3.7263 & .00916 & .10285 & .0002527 \\
\hline $57306 \ldots \ldots$ & 2.86 & 434 & 7.9772 & .01838 & .22815 & .0005257 \\
\hline $57406 \ldots$ & 2.75 & 135 & 2.4923 & .01846 & .06854 & .0005077 \\
\hline $57407 \ldots \ldots$ & 2.62 & 762 & 14.9992 & .01968 & .39297 & .0005157 \\
\hline $57408 \ldots$ & 2.61 & 596 & 12.2004 & .02047 & .31842 & .0005343 \\
\hline $57506 \ldots$ & 2.80 & 180 & 2.7616 & .01534 & .07733 & .0004296 \\
\hline $57507 \ldots$ & 2.85 & 359 & 6.9861 & .01946 & .19905 & .0005545 \\
\hline $57509 \ldots$ & 2.54 & 611 & 10.6261 & .01739 & $.26,990$ & .0004417 \\
\hline $57606 \ldots$ & 2.74 & 132 & 3.0790 & .02333 & $.08+36$ & .0006391 \\
\hline $57608 \ldots$ & 2.64 & 438 & 8.6189 & .01968 & .22756 & .0005195 \\
\hline $57805 \ldots$ & 2.87 & 270 & 4.8988 & .01814 & .14060 & .0005207 \\
\hline $58206 \ldots$ & 2.67 & 148 & 1.3961 & .00943 & .03728 & .0002519 \\
\hline $58505 \ldots$ & 2.95 & 273 & 7.4516 & .02730 & .21982 & .0008052 \\
\hline $58805 \ldots$ & 2.74 & 1,158 & 23.1471 & .01999 & .63422 & .0005464 \\
\hline $63106 .$. & 2.79 & 165 & 3.3006 & .02001 & .09208 & .0005581 \\
\hline $66005 \ldots$ & 2.63 & 370 & 7.6690 & .02073 & .20170 & .0005451 \\
\hline $69506 \ldots$ & 2.50 & 663 & 13.5696 & .02047 & .33923 & .0005117 \\
\hline $69705 \ldots \ldots$ & 2.50 & 244 & 3.7810 & .01550 & .09453 & .0003874 \\
\hline $72106 \ldots \ldots$ & 2.95 & 430 & 8.2929 & .01929 & $.2+464$ & .0005689 \\
\hline $73308 \ldots$ & 2.92 & 624 & 14.2986 & .02291 & .41752 & .0006539 \\
\hline $74506 \ldots$ & 2.73 & 23 & .4096 & .01781 & .01118 & .0004862 \\
\hline $74508 \ldots$ & 2.60 & 57 & .8172 & .01434 & .02125 & .0003728 \\
\hline $74605 \ldots$ & 2.60 & 399 & 7.1181 & .01784 & .18507 & .0004638 \\
\hline
\end{tabular}


TABLE 8.-Analyses of plants, arranged according to percentage of proteid nitrogen. Crop of 1903-Continued.

2.5 TO 3 PER CEN'T PR@TEID NITROGEN-Continued.

\begin{tabular}{|c|c|c|c|c|c|c|}
\hline \multirow{2}{*}{$\begin{array}{c}\text { Record num- } \\
\text { ber. }\end{array}$} & \multirow{2}{*}{$\begin{array}{l}\text { Percent- } \\
\text { age of } \\
\text { proteid } \\
\text { nitrogen } \\
\text { in kernels. }\end{array}$} & \multirow{2}{*}{$\begin{array}{c}\text { Number } \\
\text { of ker- } \\
\text { nels per } \\
\text { plant. }\end{array}$} & \multicolumn{2}{|c|}{ Weight (in grams) of- } & \multirow{2}{*}{$\begin{array}{l}\text { Total pro- } \\
\text { teid nitro- } \\
\text { gen in all } \\
\text { kernels } \\
\text { (gram). }\end{array}$} & \multirow{2}{*}{$\begin{array}{c}\text { Proteid } \\
\text { nitrogen in } \\
\text { average ker- } \\
\text { nel } \\
\text { (gram). }\end{array}$} \\
\hline & & & $\begin{array}{c}\text { Kernels } \\
\text { per plant. }\end{array}$ & $\begin{array}{c}\text { Average } \\
\text { kernel. }\end{array}$ & & \\
\hline $74607 \ldots \ldots$ & . $\quad 2.56$ & 491 & 8. 3406 & $0.01699^{-}$ & 0.21352 & 0.0004349 \\
\hline $81405 \ldots . .$. & 2.62 & 240 & 4.5737 & .01862 & .11710 & .0004879 \\
\hline $81505 \ldots . .$. & 2.94 & 146 & 2.8327 & .01940 & .08328 & .0005704 \\
\hline $81706 \ldots . .$. & 2.71 & 722 & 15.3928 & .02132 & .41715 & .0005778 \\
\hline $85205 \ldots \ldots \ldots$ & 2.60 & 214 & 3.4766 & .01625 & .09039 & .0004224 \\
\hline $85206 \ldots \ldots .$. & 2. 66 & 376 & 4.9315 & .01312 & .13118 & .0003332 \\
\hline $86105 \ldots \ldots \ldots$ & 2.56 & 203 & 3.0282 & .01495 & .07964 & .0003923 \\
\hline $86106 \ldots \ldots$ & 2.63 & 436 & 7. 6241 & .01749 & .20052 & .0004599 \\
\hline $88605 \ldots \ldots$ & 2.80 & 69 & 1. 6362 & .02731 & .04581 & .0007640 \\
\hline $88606 \ldots$ & 2.53 & 481 & 9.9456 & .02068 & .25162 & .0005231 \\
\hline $88607 \ldots \ldots$ & 2.61 & 234 & 5.1584 & .02205 & .13463 & .0005754 \\
\hline $88905 \ldots \ldots$ & 2.83 & 293 & 5. 3069 & .01811 & .15019 & .0005126 \\
\hline $88906 \ldots . .$. & 2.65 & 546 & 9.9034 & .01814 & .26245 & .0004807 \\
\hline $91906 \ldots$ & 2.81 & 200 & 3.5486 & .01774 & .09972 & .0004986 \\
\hline $92205 \ldots \ldots \ldots$ & 2.74 & 345 & 5.2616 & .01525 & .14417 & .0004179 \\
\hline $92206 \ldots \ldots \ldots$ & 2.67 & 46 & 1. 1074 & .02407 & .02957 & .0006428 \\
\hline $92207 \ldots \ldots \ldots$ & 2.55 & 209 & 3.6926 & .01767 & .09416 & .0004505 \\
\hline $92208 \ldots \ldots$ & 2.72 & 353 & 6.6206 & .01876 & .18008 & .0005102 \\
\hline $92305 \ldots \ldots \ldots$ & 2.93 & 160 & 2.3859 & .01491 & .06991 & .0004369 \\
\hline $92408 \ldots \ldots$. & 2.97 & 207 & 3.7820 & .01827 & .11233 & .0005426 \\
\hline $92507 \ldots \ldots$ & 2.58 & 505 & 9.6779 & .01916 & .24969 & .0004944 \\
\hline $94206 \ldots . .$. & 2.78 & 402 & 7.5006 & .01866 & .20851 & .0005187 \\
\hline $94207 \ldots \ldots$. & 2.86 & 718 & 13.7057 & .01909 & .39199 & .0005460 \\
\hline $94907 \ldots \ldots \ldots$ & 2.94 & 626 & 12. 1918 & .01948 & .35844 & .0005726 \\
\hline $95505 \ldots \ldots$ & 2.81 & 37 & .3146 & .00850 & .00884 & .0002389 \\
\hline $95506 \ldots \ldots$ & 2.74 & 597 & 11.0548 & .01852 & .30291 & .0005074 \\
\hline $95507 \ldots \ldots \ldots$ & 2.59 & 571 & 12.1592 & .02030 & .31492 & .0005515 \\
\hline $95508 \ldots \ldots \ldots$ & 2.56 & 740 & 14.4617 & .01954 & .37023 & .0005003 \\
\hline $95705 \ldots \ldots \ldots$ & 2.54 & 636 & 10.3426 & .01626 & .26270 & .0004131 \\
\hline $95706 \ldots \ldots . .$. & 2.73 & 267 & 5. 1629 & .01934 & .14095 & .0005279 \\
\hline Average .... & 2.731 & 370.36 & 7.1755 & .019354 & .194423 & .00052706 \\
\hline
\end{tabular}

3 TO 3.5 PER CENT PROTEID NITROGEN.

\begin{tabular}{|c|c|c|c|c|c|c|}
\hline $17305 \ldots \ldots \ldots$ & 3.03 & 183 & 3. 6302 & 0.01984 & 0.10999 & 0.0006010 \\
\hline $17306 \ldots \ldots$ & 3.09 & 243 & 3.9968 & .01645 & .12350 & .0005082 \\
\hline $17307 \ldots \ldots$ & 3.46 & 138 & 3. 1454 & .02280 & .10883 & .0007886 \\
\hline $17308 \ldots \ldots \ldots$ & 3.25 & 61 & 1.2275 & .02012 & .03994 & .0006540 \\
\hline $17406 \ldots \ldots$ & 3.29 & 124 & 2.0907 & .01683 & .06878 & .0005547 \\
\hline $18906 \ldots . . .$. & 3.48 & 65 & .9229 & .01420 & .03212 & .0004941 \\
\hline $20705 \ldots \ldots$. & 3.09 & 109 & 1.8517 & .01698 & .05722 & .0005249 \\
\hline $20709 \ldots . . .$. & 3.05 & 258 & 5. 3229 & .02063 & .16235 & .0006292 \\
\hline $20805 \ldots . . .$. & 3.32 & 697 & 14.6942 & .02157 & .48784 & .0006999 \\
\hline $21205 \ldots \ldots$ & 3.16 & 123 & 2.3642 & .01922 & .07471 & .0006074 \\
\hline $21208 \ldots \ldots \ldots$ & 3.24 & 287 & 5. 1594 & .01798 & .16712 & .0005824 \\
\hline $21211 \ldots \ldots \ldots$ & 3.15 & 10 & .2806 & .02806 & .00884 & .0008839 \\
\hline 21307 & 3.04 & 143 & 2.5691 & .01796 & .07810 & .0005461 \\
\hline $21308 \ldots . .$. & 3.45 & 354 & 5.8080 & .01641 & .20038 & .0005660 \\
\hline $21906 \ldots \ldots \ldots$ & 3. 18 & 408 & 10.4800 & .02563 & .33403 & .0008168 \\
\hline $21907 \ldots . .$. & 3.35 & 158 & 2.9248 & . .01851 & $.09^{-9} 98$ & .0006201 \\
\hline $21913 \ldots \ldots \ldots$ & 3.01 & 492 & 10.1925 & .02072 & .30680 & .0006235 \\
\hline $22206 \ldots \ldots . . .$. & 3.22 & 146 & 2.5712 & .01720 & .08086 & .0005538 \\
\hline $22208 \ldots \ldots \ldots$ & 3.18 & 118 & 1.9090 & .01619 & .06071 & .0005144 \\
\hline $22210 \ldots \ldots \ldots$ & 3.17 & 298 & 6.0173 & .02019 & .19075 & .0006401 \\
\hline $22211 \ldots \ldots \ldots$ & 3.17 & 561 & $11.56^{75}$ & .02062 & .36671 & .0006537 \\
\hline $26105 \ldots \ldots \ldots$ & 3.02 & 131 & 1.8242 & .01393 & .05508 & .0003662 \\
\hline $26808 \ldots \ldots \ldots$ & 3.09 & 222 & 3.8811 & .01748 & .11992 & .0005402 \\
\hline $27507 \ldots \ldots \ldots$ & 3.08 & 75 & 1. 3746 & .01833 & .04234 & .0005646 \\
\hline $28206 \ldots \ldots \ldots$ & 3.07 & 219 & 4. 3698 & .01996 & .13415 & .0006126 \\
\hline $28806 \ldots \ldots . .$. & 3.02 & 685 & 14.4630 & .02111 & .43679 & .0006376 \\
\hline $32207 \ldots \ldots \ldots$ & 3.48 & 69 & 1. $25^{73}$ & .01822 & .04375 & .0006341 \\
\hline $33305 \ldots . . .$. & 3,41 & 150 & 3. 1346 & .02090 & .10689 & .0007126 \\
\hline $33607 \ldots \ldots \ldots$ & 3.22 & 136 & 2.8903 & .02125 & .09307 & .0006843 \\
\hline $34606 \ldots . . .$. & 3.12 & 280 & 6. 1962 & .02213 & .19332 & .0006904 \\
\hline $39507 \ldots \ldots \ldots$ & 3.02 & 111 & 1.8862 & .01699 & .05696 & .0005132 \\
\hline $40305 \ldots \ldots \ldots$ & 3.11 & 179 & 3. 6003 & .02011 & .11197 & .0006255 \\
\hline $40405 \ldots . .$. & 3.17 & 46 & .6316 & .01373 & .02002 & .0004352 \\
\hline $42405 \ldots \ldots \ldots$ & 3.07 & 66 & 1.4892 & .02251 & .04572 & .0006927 \\
\hline $42905 \ldots . .$. & 3.17 & 67 & 1.2499 & .01866 & .03650 & .0005447 \\
\hline $46105 \ldots . .$. & 3.00 & 260 & 4. 6146 & .01775 & .13843 & .0005324 \\
\hline $48306 \ldots \ldots \ldots$ & 3.29 & 157 & 2.6571 & .01692 & .08742 & .0005568 \\
\hline
\end{tabular}


TABLE 8.-Analyses of plants, arranged according to percentage of proteid nitrogen. Crop of 1903-Continued.

3 TO 3.5 PER CENT PROTEID NITROGEN-Continued.

\begin{tabular}{|c|c|c|c|c|c|c|}
\hline \multirow{2}{*}{$\begin{array}{c}\text { Record num- } \\
\text { ber. }\end{array}$} & \multirow{2}{*}{$\begin{array}{l}\text { Percent- } \\
\text { age of } \\
\text { proteid } \\
\text { nitrogen } \\
\text { in kernels. }\end{array}$} & \multirow{2}{*}{$\begin{array}{l}\text { Number } \\
\text { of ker- } \\
\text { nels per } \\
\text { plant. }\end{array}$} & \multicolumn{2}{|c|}{ Weight (in grams) of- } & \multirow{2}{*}{$\begin{array}{l}\text { Total pro- } \\
\text { teid nitro- } \\
\text { gen in all } \\
\text { kernels } \\
\text { (gram). }\end{array}$} & \multirow{2}{*}{$\begin{array}{c}\text { Proteid } \\
\text { nitrogen in } \\
\text { average ker- } \\
\text { nel } \\
\text { (gram). }\end{array}$} \\
\hline & & & $\begin{array}{c}\text { Kernels } \\
\text { per plant. }\end{array}$ & $\begin{array}{l}\text { Average } \\
\text { kernel. }\end{array}$ & & \\
\hline $48405 \ldots \ldots \ldots$ & 3.31 & 76 & 0.9701 & 0.01276 & 0.03211 & 0.0004225 \\
\hline $48506 \ldots \ldots$ & 3.20 & 556 & 9.4585 & .01701 & .30267 & .0005444 \\
\hline $48705 \ldots \ldots$. & 3.13 & 264 & 4.3615 & .01652 & .13652 & .0005171 \\
\hline $48706 \ldots$ & 3.00 & 379 & 6.1986 & .01635 & . 18596 & .0004906 \\
\hline $49505 \ldots$ & 3.24 & 67 & 1.2716 & .01898 & .04120 & .0006149 \\
\hline $50905 \ldots$ & 3.30 & 221 & 2.3982 & .01085 & .07914 & .0003581 \\
\hline $55005 \ldots \ldots \ldots$ & 3.05 & 393 & 7.9684 & .02028 & .24304 & .0006185 \\
\hline $55006 \ldots \ldots$. & 3.16 & 451 & 7.1852 & .01593 & .22705 & .0005034 \\
\hline $55205 \ldots \ldots$. & 3.10 & 40 & .6893 & .01723 & .02137 & .0005342 \\
\hline $55508 \ldots$ & 3.11 & 216 & 3.7407 & .01732 & .11636 & .0005386 \\
\hline $57305 \ldots \ldots \ldots$ & 3.19 & 501 & 8.5777 & .01666 & .29188 & .0005326 \\
\hline $57905 \ldots \ldots \ldots$ & 3.18 & 221 & 2.4731 & .01118 & .07859 & .0003556 \\
\hline $58207 \ldots \ldots$. & 3.09 & 307 & 4. 2207 & .01375 & .13042 & .0004248 \\
\hline $58705 \ldots \ldots \ldots$ & 3.01 & 235 & 2.5436 & .01082 & .07656 & .0003256 \\
\hline $62805 \ldots . .$. & 3.25 & 111 & 1. 3451 & .01212 & .04272 & .0003938 \\
\hline $63105 \ldots \ldots \ldots$ & 3.24 & 90 & 1.5452 & .01717 & .05007 & .0005563 \\
\hline $72405 \ldots \ldots \ldots$ & 3.36 & 213 & 8.4415 & .03963 & .28363 & .0013316 \\
\hline $72707 \ldots \ldots \ldots$ & 3.49 & 225 & 4.5806 & .02036 & .15986 & .0007105 \\
\hline $72806 \ldots \ldots \ldots$ & 3.01 & 110 & 2.0970 & .01906 & .06312 & .0005738 \\
\hline $74507 \ldots \ldots \ldots$ & 3.02 & 493 & 9.2130 & .01869 & .27823 & .0005644 \\
\hline $81406 \ldots \ldots .$. & 3.31 & 72 & 1. 2391 & .01721 & .04101 & .0005697 \\
\hline $84906 \ldots . . .$. & 3.43 & 382 & 7.5438 & .01975 & .25873 & .0006773 \\
\hline $91305 \ldots \ldots \ldots$ & 3.21 & 138 & 3.0940 & .02242 & .09932 & .0007197 \\
\hline $91905 \ldots . . . . .$. & 3.36 & 198 & 3.4436 & .01739 & .11570 & .0005844 \\
\hline $92405 \ldots$ & 3.10 & 214 & 3.4356 & .01605 & .10650 & .0004977 \\
\hline $92406 \ldots$ & 3.11 & 380 & 8.2366 & .02168 & .25616 & .0006741 \\
\hline $92505 \ldots$ & 3.00 & 156 & 2.6615 & .01706 & .07985 & .0005118 \\
\hline $94208 \ldots \ldots \ldots$ & 3.10 & 322 & 3.7828 & .01175 & .11727 & .0003642 \\
\hline $94906 \ldots$ & 3.41 & 685 & 12.3862 & .01808 & .42236 & .0006166 \\
\hline Average. & 3.184 & 235.5 & 4.38558 & .018366 & .139656 & .00058156 \\
\hline
\end{tabular}

3.5 TO 4 PER CENT PROTEID NITROGEN.

\begin{tabular}{|c|c|c|c|c|c|c|}
\hline $17506 \ldots \ldots$ & 3.52 & 93 & 2.2881 & 0.02460 & 0.08044 & 0.0008660 \\
\hline $17507 \ldots \ldots$ & 3.80 & 43 & .7220 & .01795 & .02933 & .0006822 \\
\hline $18905 \ldots \ldots$ & 3.81 & 103 & 1.4864 & .01443 & .05663 & .0005498 \\
\hline $21209 \ldots \ldots$ & 3.61 & 89 & 1.4484 & .01627 & .05228 & .0005875 \\
\hline $21811 \ldots \ldots \ldots$ & 3.75 & 567 & 11.9114 & .02101 & .44666 & .0007877 \\
\hline $21908 \ldots \ldots \ldots$ & 3.82 & 173 & 3.5574 & .02056 & .13589 & .0007855 \\
\hline $22209 \ldots \ldots$ & 3.84 & 31 & .4336 & .01399 & .01665 & .0005371 \\
\hline $26107 \ldots \ldots$ & 3.92 & 144 & 2.0390 & .01416 & .07993 & .0005551 \\
\hline $32608 \ldots \ldots$ & 3.78 & 55 & 1.0183 & .01851 & .03849 & .0006998 \\
\hline $34206 \ldots \ldots$ & 3.73 & 81 & 1.5940 & .01968 & .05946 & .0007340 \\
\hline $36905 \ldots \ldots$ & 3.88 & 267 & 5.0200 & .01880 & .19478 & .0007295 \\
\hline $38505 \ldots \ldots$ & 3.61 & 563 & 12.1088 & .02252 & .43713 & .0007764 \\
\hline $42205 \ldots \ldots$ & 3.63 & 94 & 1.8494 & .01967 & .06713 & .0007142 \\
\hline $45005 \ldots \ldots$ & 3.58 & 235 & 3. 2340 & .01376 & .11575 & .0004927 \\
\hline $48505 \ldots \ldots$ & 3.66 & 137 & 1.9154 & .01398 & .07010 & .0005117 \\
\hline $49905 \ldots \ldots \ldots$ & 3.62 & 23 & .6760 & .02939 & .02436 & .0010640 \\
\hline $50705 \ldots \ldots$ & 3.54 & 30 & .5958 & .01986 & .02109 & .0007032 \\
\hline $50906 \ldots \ldots$ & 3.57 & 114 & 1.7280 & .01516 & .06169 & .0005411 \\
\hline $66006 \ldots \ldots$ & 3.54 & 366 & 6.0090 & .01642 & .21272 & .0005812 \\
\hline $66008 \ldots \ldots$. & 3.59 & 174 & 3.1555 & .01814 & .11328 & .0006510 \\
\hline $72706 \ldots \ldots$ & 3.86 & 591 & 14.6802 & .02484 & .56666 & .0009588 \\
\hline $94909 \ldots \ldots$ & 3.60 & 218 & 3.6977 & .01696 & .13312 & .0006106 \\
\hline Average.... & 3.69 & 190.5 & 3.68947 & .018666 & .13698 & .00068723 \\
\hline
\end{tabular}


TABLE 8.-Analyses of plants, arranged according to percentage of proteid nitrogen. Crop of 1903-Continued.

4 TO 4.5 PER CENT PROTEID NITROGEN.

\begin{tabular}{|c|c|c|c|c|c|c|}
\hline \multirow{2}{*}{$\begin{array}{c}\text { Record num- } \\
\text { ber. }\end{array}$} & \multirow{2}{*}{$\begin{array}{l}\text { Percent- } \\
\text { age of } \\
\text { proteid } \\
\text { nitrogen } \\
\text { in kernels. }\end{array}$} & \multirow{2}{*}{$\begin{array}{l}\text { Number } \\
\text { of ker- } \\
\text { nels per } \\
\text { plant. }\end{array}$} & \multicolumn{2}{|c|}{ Weight (in grams) of - } & \multirow{2}{*}{$\begin{array}{l}\text { Total pro- } \\
\text { teid nitro- } \\
\text { gen in all } \\
\text { kernels } \\
\text { (gram). }\end{array}$} & \multirow{2}{*}{$\begin{array}{c}\text { Proteid } \\
\text { nitrogen in } \\
\text { average ker- } \\
\text { nel } \\
\text { (gram). }\end{array}$} \\
\hline & & & $\begin{array}{c}\text { Kernels } \\
\text { per plant. }\end{array}$ & $\begin{array}{l}\text { Average } \\
\text { kernel. }\end{array}$ & & \\
\hline 21812 & 4.26 & 983 & 14.8139 & 0.01507 & 0.63107 & 0.0006420 \\
\hline 21813 & 4.04 & 216 & 4.0258 & 0.0 & .16377 & .0007582 \\
\hline 21909 & 4.4 & 52 & 12.18 & .0 & .53 & .0010265 \\
\hline 27308 & 4.1 & 25 & 4.512 & .01777 & .18726 & .0007373 \\
\hline 344 & 4. & 20 & 4. & .019 & .178 & .0008635 \\
\hline 43 & 4. & 93 & 1.4464 & .015 & .05974 & .0006423 \\
\hline & 4. & 44 & .75 & .017 & .03148 & .0007155 \\
\hline 55007 & 4.2 & 118 & 2.1571 & .018 & .09082 & .0007696 \\
\hline 6930 & 4. & 103 & 2.0430 & .019 & .0903 & .0008767 \\
\hline 7620 & & 44 & 5.44 & .012 & .242 & .0005417 \\
\hline 92506 . & 4. & 229 & 3.8709 & .016 & .16993 & .0007421 \\
\hline Average. & 4.27 & 292.6 & 5.03397 & .017689 & .21674 & .00075594 \\
\hline
\end{tabular}

MORE THAN 4.5 PER CENT PROTEID NITROGEN

\begin{tabular}{|c|c|c|c|c|c|c|}
\hline $17505 \ldots \ldots$ & 4.70 & 29 & 0.3885 & 0.01340 & 0.01826 & 0.0006296 \\
\hline $21206 \ldots . . .$. & 5.23 & 149 & 2.8564 & .01917 & .14939 & .0010026 \\
\hline $21210 \ldots . .$. & 5.03 & 237 & 3.9143 & .01578 & .19689 & .0007934 \\
\hline $21706 \ldots . . .$. & 4.71 & 807 & 19.3318 & .02390 & .91052 & .0011283 \\
\hline $21911 \ldots . . .$. & 5.48 & 383 & 8.4593 & .02209 & .46356 & .0012103 \\
\hline $38605 \ldots \ldots \ldots$ & 5.85 & 61 & 1.2124 & .01988 & .07093 & .0011627 \\
\hline $38607 \ldots \ldots \ldots$ & 4.55 & 19 & .3037 & .01598 & .01382 & .0007273 \\
\hline $40205 \ldots$. & 4.69 & 194 & 3.6302 & .01871 & .17026 & .0008776 \\
\hline $48406 \ldots \ldots$. & 4.87 & 249 & 3.2964 & .01324 & .16053 & .0006447 \\
\hline $65305 \ldots \ldots \ldots$ & 4.92 & 78 & 1.8018 & .02310 & .08865 & .0011365 \\
\hline $69805 \ldots . . .$. & 5.82 & 110 & 2.4420 & .02220 & .14213 & .0012921 \\
\hline $72605 \ldots$ & 4.65 & 65 & 1.1166 & .01718 & .05192 & .0007988 \\
\hline $72607 \ldots \ldots$ & 5.59 & 188 & 3.4442 & .01832 & .19253 & .0010241 \\
\hline $92306 . . . . .$. & 4.93 & 347 & 6.0091 & .01732 & .29625 & .0008539 \\
\hline A verage. & 5.07 & 208.28 & 4.15727 & .01859 & .208974 & .0009487 \\
\hline
\end{tabular}

TABLE 9.-SUmmary of analyses of plants, arranged according to percentage of proteid nitrogen. Crop of 1903.

\begin{tabular}{|c|c|c|c|c|c|c|c|}
\hline \multirow{2}{*}{$\begin{array}{l}\text { Range of per- } \\
\text { centage of proteid } \\
\text { nitrogen. }\end{array}$} & \multirow{2}{*}{$\begin{array}{l}\text { Percent- } \\
\text { age of } \\
\text { proteid } \\
\text { nitrogen } \\
\text { in ker- } \\
\text { nels. }\end{array}$} & \multicolumn{2}{|c|}{ Number of- } & \multicolumn{2}{|c|}{$\begin{array}{c}\text { Weight (in grams) } \\
\text { of- }\end{array}$} & \multicolumn{2}{|c|}{$\begin{array}{l}\text { Proteid nitrogen } \\
\text { (in grams) in- }\end{array}$} \\
\hline & & $\begin{array}{l}\text { Analy- } \\
\text { ses. }\end{array}$ & $\begin{array}{l}\text { Ker- } \\
\text { nels. }\end{array}$ & Kernels. & $\begin{array}{l}\text { Average } \\
\text { kernel. }\end{array}$ & $\begin{array}{l}\text { All ker- } \\
\text { nels. }\end{array}$ & $\begin{array}{l}\text { Average } \\
\text { kernel. }\end{array}$ \\
\hline $\begin{array}{l}1 \text { to } 2 . \ldots \ldots \ldots \\
2 \text { to } 2.5 \ldots \ldots \ldots \\
2.5 \text { to } 3 \ldots \ldots \ldots \\
3 \text { to } 3.5 \ldots \ldots \ldots \\
3.5 \text { to } 4 \ldots \ldots \ldots \\
4 \text { to } 4.5 \ldots \ldots \ldots \\
4.5 \text { and } \text { over.... }\end{array}$ & $\begin{array}{l}1.749 \\
2.32 \\
2.73 \\
3.18 \\
3.69 \\
4.27 \\
5.07\end{array}$ & $\begin{array}{r}28 \\
65 \\
145 \\
66 \\
22 \\
11 \\
14\end{array}$ & $\begin{array}{l}320.3 \\
396 \\
370 \\
235 \\
190 \\
292 \\
208\end{array}$ & $\begin{array}{l}6.2382 \\
8.2502 \\
7.1755 \\
4.3856 \\
3.6895 \\
5.0340 \\
4.1573\end{array}$ & $\begin{array}{r}0.01871 \\
.02011 \\
.01935 \\
.01837 \\
.01867 \\
.01769 \\
.01859\end{array}$ & $\begin{array}{r}0.10655 \\
.19032 \\
.19442 \\
.13966 \\
.13698 \\
.21674 \\
.20897\end{array}$ & $\begin{array}{r}0.0003291 \\
.0004660 \\
.0005271 \\
.0005816 \\
.0006872 \\
.0007559 \\
.0009487\end{array}$ \\
\hline
\end{tabular}

Table 10 shows the analyses of the crop of 1903 arranged on the basis of weight of average kernel. Determinations of gliadin and glutenin were made in these analyses and the sums of these are inserted in this table. ${ }^{a}$ All plants having an average kernel weight

$a$ Determinations of gliadin and glutenin were made by methods practically the same as those described by Prof. Harry Snyder in Bulletin No. 63 of the Minnesota Experiment Station, except that smaller quantities were used. 
of less than 0.010 gram form the first class and each succeeding clas; increases by 0.002 gram. Table 11 is a summary of these analyses. TABLE 10.-Analyses of plants, arranged according to weight of average kernel. Crop of 1903.

WEIGHT OF AVERAGE KERNEL, 0.000 TO 0.010 GRAM.

\begin{tabular}{|c|c|c|c|c|c|c|c|c|c|}
\hline \multirow{2}{*}{$\begin{array}{l}\text { Record } \\
\text { number. }\end{array}$} & \multirow{2}{*}{$\begin{array}{c}\text { Weight } \\
\text { of aver- } \\
\text { age } \\
\text { kernel } \\
\text { (gram). }\end{array}$} & \multirow{2}{*}{$\begin{array}{l}\text { Num- } \\
\text { ber of } \\
\text { kernels } \\
\text { on } \\
\text { plant. }\end{array}$} & \multirow{2}{*}{$\begin{array}{l}\text { Weight } \\
\text { of kernels } \\
\text { on plant } \\
\text { (grams). }\end{array}$} & \multirow{2}{*}{$\begin{array}{l}\text { Per- } \\
\text { centage } \\
\text { of pro- } \\
\text { teid ni- } \\
\text { trogen } \\
\text { in ker- } \\
\text { nels. }\end{array}$} & \multicolumn{2}{|c|}{$\begin{array}{l}\text { Proteid nitrogen } \\
\text { (gram) in- }\end{array}$} & \multirow{2}{*}{$\begin{array}{l}\text { Percent- } \\
\text { age of } \\
\text { gliadin- } \\
\text { plus-glu- } \\
\text { tenin ni- } \\
\text { trogen in } \\
\text { kernels. }\end{array}$} & \multicolumn{2}{|c|}{$\begin{array}{l}\text { Gliadin-plus-glu- } \\
\text { tenin nitrogen } \\
\text { (gram) in- }\end{array}$} \\
\hline & & & & & $\begin{array}{l}\text { Average } \\
\text { kernel. }\end{array}$ & $\begin{array}{l}\text { Kernels } \\
\text { on plant. }\end{array}$ & & $\begin{array}{l}\text { Average } \\
\text { kernel. }\end{array}$ & $\begin{array}{l}\text { Kernels } \\
\text { on plant. }\end{array}$ \\
\hline $\begin{array}{l}22205 . . \\
57105 . . \\
58206 . . \\
95505 .\end{array}$ & $\begin{array}{r}0.00953 \\
.00916 \\
.00943 \\
.00850\end{array}$ & $\begin{array}{r}283 \\
407 \\
148 \\
37\end{array}$ & $\begin{array}{r}2.6965 \\
-3.7263 \\
1.3961 \\
.3146\end{array}$ & $\begin{array}{l}2.81 \\
2.76 \\
2.67 \\
2.81\end{array}$ & $\begin{array}{r}0.0002677 \\
.0002527 \\
.0002519 \\
.0002389\end{array}$ & $\begin{array}{r}0.07577 \\
.10285 \\
.03728 \\
.00884\end{array}$ & & $\begin{array}{l}0.0001877 \\
\ldots \ldots \ldots\end{array}$ & $\begin{array}{c}0.05312 \\
\ldots \ldots \ldots \ldots\end{array}$ \\
\hline A verage. & .00915 & 219 & 2.0334 & 2.76 & 2528 & 18 & 1.97 & .0001877 & .05312 \\
\hline
\end{tabular}

WEIGHT OF AVERAGE KERNEL, 0.010 TO 0.012 GRAM.

\begin{tabular}{|c|c|c|c|c|c|c|c|c|c|}
\hline $37906 .$. & 0.01086 & 19 & 0.2063 & 2.44 & 0.0002649 & 0.00503 & & $\ldots$ & $\ldots$ \\
\hline $45605 \ldots$. & .01161 & 61 & .7081 & 2.82 & .0003273 & .01997 & - & $\ldots$ & $\ldots$ \\
\hline $5090 ; \ldots .$. & .01085 & 221 & 2.3982 & 3.30 & .0003581 & .07914 & -.... & $\ldots$ & \\
\hline $57905 \ldots$. & .01118 & 221 & 2. 4731 & 3.18 & .0003556 & .07859 & 2.92 & 0.0003264 & 0.07221 \\
\hline $58705 \ldots . .$. & .01082 & 235 & 2.5436 & 3.01 & .0003258 & .07656 & 2.47 & .0002673 & .06283 \\
\hline $94208 \ldots .$. & .01175 & 322 & 3.7828 & 3.10 & .0003642 & .11727 & ........... & $\ldots \ldots \ldots$. & $\ldots \ldots \ldots$ \\
\hline Average. & .01118 & 179 & 2.0187 & 2.98 & .0003326 & .06276 & 2. 69 & .0002968 & .06752 \\
\hline
\end{tabular}

WEIGHT OF AVERAGE KERNEL, 0.012 TO 0.014 GRAM.

\begin{tabular}{|c|c|c|c|c|c|c|c|c|c|}
\hline 175 & 0.01340 & 29 & 0.3885 & 4.70 & 0.0006296 & 0.01826 & & & \\
\hline 222 & .01399 & 31 & .4336 & 3.84 & .0005371 & .01665 & & & \\
\hline 26105. & .01393 & 131 & 1.8242 & 3.02 & .0003662 & .05508 & & & \\
\hline 39606. & .01341 & 346 & 4.6383 & 2.37 & .0003177 & .10967 & & & \\
\hline 40405. & .01373 & 46 & .6316 & 3.17 & .0004352 & .02002 & & & \\
\hline 422 & .01264 & 25 & .3161 & 1.46 & .0001846 & .00462 & & & 004900 \\
\hline 45005. & .01376 & 235 & 3.2340 & 3.5 & .0004927 & .11575 & 1.36 & 0.0001871 & 0.04398 \\
\hline 458 & .01234 & 124 & 1.5298 & 1.8 & .0002700 & .02815 & ... & ..... & .......... \\
\hline $\begin{array}{l}48405 . \\
48406\end{array}$ & $\begin{array}{r}.01276 \\
.01324\end{array}$ & $\begin{array}{r}76 \\
249\end{array}$ & $\begin{array}{r}.9701 \\
3.2964\end{array}$ & $\begin{array}{l}3.31 \\
4.87\end{array}$ & .0004225 & $\begin{array}{r}.03211 \\
.16053\end{array}$ & 2.25 & .0002979 & 08168 \\
\hline 48408 & .01291 & 27 & .3485 & 2.81 & .0003627 & .00979 & & & \\
\hline 48505 . & .01398 & 137 & 1.9154 & 3.66 & .0005117 & .07010 & 1.76 & .0002460 & .03371 \\
\hline 507 & .01343 & 35 & $\therefore 4701$ & 2.80 & .0003761 & .01316 & 2,49 & 0003424 & 10510 \\
\hline $\begin{array}{l}58207 \\
58905\end{array}$ & $\begin{array}{l}.01375 \\
.01355\end{array}$ & $\begin{array}{l}307 \\
170\end{array}$ & $\begin{array}{l}4.220 \pi \\
2.3031\end{array}$ & $\begin{array}{l}3.09 \\
2.43\end{array}$ & $\begin{array}{r}.0004248 \\
.0003292\end{array}$ & $\begin{array}{l}.13042 \\
.05596\end{array}$ & 2.49 & .0003424 & .10510 \\
\hline $62805 .$. & .01212 & 111 & 1.3.51 & 3.25 & .0003938 & .04272 & . & . & $\cdots$ \\
\hline 76206 .. & .01217 & 447 & 5.4411 & 4.45 & .0005417 & .24213 & 2.03 & .0002471 & .11046 \\
\hline $\begin{array}{l}85206 . . \\
94605 .\end{array}$ & $\begin{array}{l}.01312 \\
.01307\end{array}$ & $\begin{array}{r}376 \\
56\end{array}$ & $\begin{array}{r}4.9315 \\
.7319\end{array}$ & $\begin{array}{l}2.66 \\
1.95\end{array}$ & $\begin{array}{r}.0003332 \\
.0002549\end{array}$ & $\begin{array}{l}.13118 \\
.01427\end{array}$ & .. & ..... & ... \\
\hline & & & & & & & & & \\
\hline Averag & .01323 & 155.7 & 2.0510 & .12 & .0004120 & .06687 & .98 & 0002641 & 499 \\
\hline
\end{tabular}

WEIGHT OF AVERAGE KERNEL, 0.014 TO 0.016 GRAM.

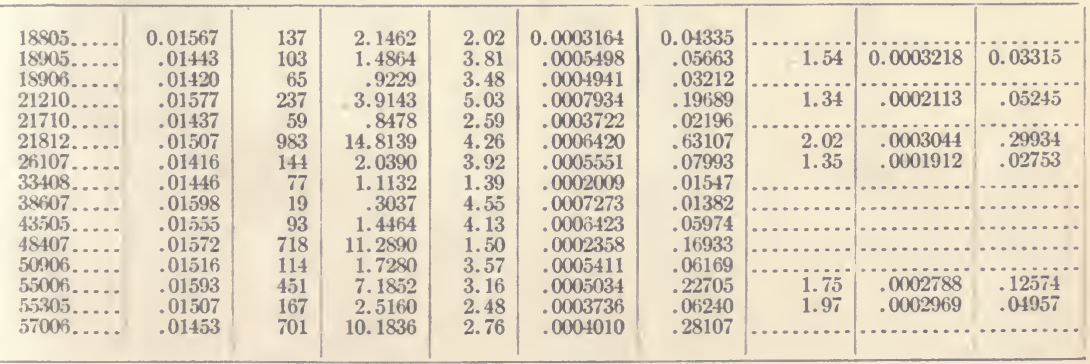


T:xis 10.-Analyses of plants, arranged according to weight of average kernel. Crop of 1903-Continued.

WEIGHT OF AVERAGE KERNEL, 0.014 TO 0.016 GRAM-Continued.

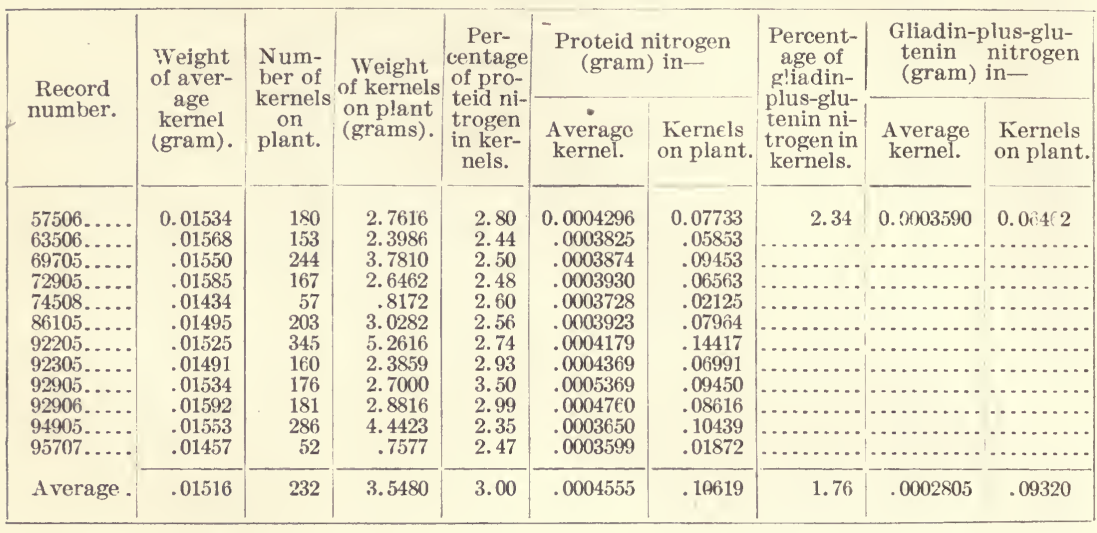

WEIGHT OF AVERAGE KERNEL, 0.016 TO 0.018 GRAM.

\begin{tabular}{|c|c|c|c|c|c|c|c|c|c|}
\hline $17306 \ldots .$. & 0.01645 & 243 & 3.9968 & 3.09 & 0.0005082 & 0.12350 & & & \\
\hline $17406 \ldots$ & .01686 & 124 & 2.0907 & 3.29 & .0005547 & .06878 & & & \\
\hline $17507 \ldots$ & .01795 & 43 & .7720 & 3.80 & .0006822 & .02934 & & & \\
\hline $20705 \ldots .$. & .01698 & 109 & 1.8517 & 3.09 & .0005249 & .05722 & & & \\
\hline $21208 \ldots .$. & .01798 & 287 & 5. 1594 & 3.24 & .0005824 & .16712 & 2.15 & 0.003386 & 0.11093 \\
\hline $21209 \ldots .$. & .01627 & 89 & 1.4484 & 3.61 & .0005875 & .05228 & & $\ldots \ldots \ldots$. & $\ldots . . .$. \\
\hline $21307 \ldots .$. & .01796 & 143 & 2.5691 & 3.04 & .0005461 & .07810 & $\ldots$ & $\ldots$ & ........ \\
\hline $21308 \ldots$. & .01641 & 354 & 5.8080 & 3.45 & .0005660 & .20038 & ... & .......... & .... \\
\hline $21805 \ldots .$. & .01699 & 1,232 & 20.9290 & 2.69 & .0004569 & .56299 & & $\ldots$ & \\
\hline $21808 \ldots . .$. & .01708 & 1,156 & 19. 7446 & 2.57 & .0004389 & .50744 & 1.95 & .0003348 & .38700 \\
\hline $22206 \ldots . .$. & .01720 & 146 & 2.5712 & 3.22 & .0005538 & .08086 & 2.11 & .0003629 & .05425 \\
\hline $22208 \ldots . .$. & .01619 & 118 & 1.9090 & 3.18 & .0005144 & .06071 & 2.14 & .0003465 & .04084 \\
\hline $26806 \ldots .$. & .01793 & 152 & 2.7255 & 2. 60 & .0004662 & .07086 & & & \\
\hline $26808 \ldots .$. & .01748 & 222 & 3.8811 & 3.09 & .0005402 & .11992 & 2.28 & .0003935 & .08849 \\
\hline $26907 \ldots$. & .01792 & 102 & 1.8276 & 2.61 & .0004677 & .04995 & & & \\
\hline $26909 \ldots$. & .01667 & 180 & 2.9999 & 2.80 & .0004667 & .08400 & 1.88 & .0003134 & .05640 \\
\hline $27308 \ldots .$. & .01777 & 254 & 4.5123 & 4.15 & .0007373 & .18726 & $\cdots$ & ........... & ........ \\
\hline $33106 \ldots .$. & .01716 & 18 & .3089 & 2.94 & .0005045 & .00908 & & & \\
\hline 33406 . & .01627 & 283 & 4. 6045 & 2.87 & .0004670 & .13215 & & & \\
\hline 37707. & .01710 & 193 & 3.3004 & 2.93 & .0005010 & .09670 & 2.10 & .0003591 & .0 .931 \\
\hline 39507 . & .01699 & 111 & 1.8862 & 3.02 & .0005132 & .05696 & .......... & ........... & ......... \\
\hline $44505 \ldots$ & .01764 & 340 & 5.9990 & 2.94 & .0005187 & $.17 £ 37$ & $\ldots \ldots \ldots$ & & $\ldots$ \\
\hline $45705 \ldots$. & .01712 & 44 & .7532 & 4. 18 & .0007155 & .03148 & ..... & $\ldots$ & $\ldots$ \\
\hline $46105 \ldots$. & .01775 & 260 & 4.6146 & 3.00 & .0005324 & .13843 & & & \\
\hline $46107 \ldots \ldots$ & .01756 & 478 & 8.3935 & 2.54 & .0004460 & .21319 & 2.08 & .0003652 & .17458 \\
\hline $48306 \ldots .$. & .01692 & 157 & 2.6571 & 3.29 & .0005568 & .08742 & 2.13 & $.0003 c^{\circ} 04$ & .05660 \\
\hline $48505 \ldots .$. & .01701 & 556 & 9.4585 & 3.20 & .0005444 & .30267 & 2.17 & .0003691 & .20525 \\
\hline $48705 \ldots$. & .01652 & 264 & 4. 3615 & 3.13 & .0005171 & .13652 & 1.56 & .0002577 & .06804 \\
\hline $48706 \ldots .$. & .01635 & 379 & 6.1986 & 3.00 & -.0004906 & .18596 & & & -. \\
\hline $48806 \ldots . .$. & .01798 & 547 & 9.8346 & 2.70 & .0004877 & .26553 & & & \\
\hline $55205 \ldots .$. & .01723 & 40 & .6893 & 3.10 & .0005342 & .02137 & & & \\
\hline $55307 \ldots .$. & .01663 & 342 & 5. 6864 & 1.89 & .0003142 & .10747 & 1.56 & .0002594 & 08871 \\
\hline $55508 \ldots .$. & .01732 & 216 & 3. 7407 & 3.11 & .0005386 & .11636 & 1.96 & .0003395 & .07332 \\
\hline $55607 \ldots \ldots$ & .01734 & 138 & 2. 3931 & 2. 69 & .0004665 & .06437 & & $\ldots . . . .$. & \\
\hline $55905 \ldots .$. & .01751 & 331 & 5.7948 & 2.67 & .0004674 & .15470 & 1.75 & .0003064 & .10141 \\
\hline $55906 \ldots . .$. & .01603 & 499 & 7.9968 & 2.81 & .0004503 & .22471 & 1.47 & .0002356 & .11755 \\
\hline $56105 \ldots .$. & .01709 & 336 & 5. 7431 & 2. 73 & .0004667 & .15679 & 2.12 & .0003622 & .12175 \\
\hline $56107 \ldots .$. & .01658 & 872 & 14.4556 & 2.96 & .0004907 & .42790 & 2.23 & .0003697 & .32236 \\
\hline $56209 \ldots \ldots$ & .01664 & 950 & 15.8086 & 2.59 & .0004310 & .40945 & 2.21 & .0003677 & .34937 \\
\hline $57005 \ldots .$. & .01746 & 88 & 1.5364 & 2.71 & .0004731 & .04164 & 2.09 & .0003649 & .03211 \\
\hline $57305 \ldots$. & .01666 & 501 & 8.5777 & 3.19 & .0005826 & .29188 & $\ldots .$. & $\ldots \ldots \ldots$ & $\ldots \ldots \ldots$ \\
\hline $57308 \ldots .$. & .01705 & 577 & 9.8378 & 1. 69 & .0002881 & .16626 & $\ldots$ & $\ldots$. & . \\
\hline $57509 \ldots \ldots$ & .01739 & 611 & 10.6261 & 2.54 & .0004417 & .26990 & $\ldots$ & $\ldots$ & . \\
\hline $59606 \ldots . .$. & .01712 & 567 & 9.7084 & 2.16 & .0003698 & .20970 & & $\ldots$ & \\
\hline $60605 \ldots .$. & .01701 & 35 & .5952 & 1.87 & .0003180 & .01113 & $\ldots$ & . & \\
\hline $63105 \ldots . .$. & .01717 & 90 & 1.5452 & 3.24 & .0005563 & .05007 & $\ldots$ & 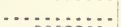 & \\
\hline $66006 \ldots .$. & .01642 & 366 & 6.0090 & 3.54 & .0005812 & .21272 & 1.38 & .0002266 & .08292 \\
\hline
\end{tabular}


TABLE 10.-Analyses of plan's, crranged according to weight of average kernel. Crop of 1903 -Continued.

WEIGHT OF AVERAGE KERNEL, 0.016 TO 0.018 GRAM-Continued.

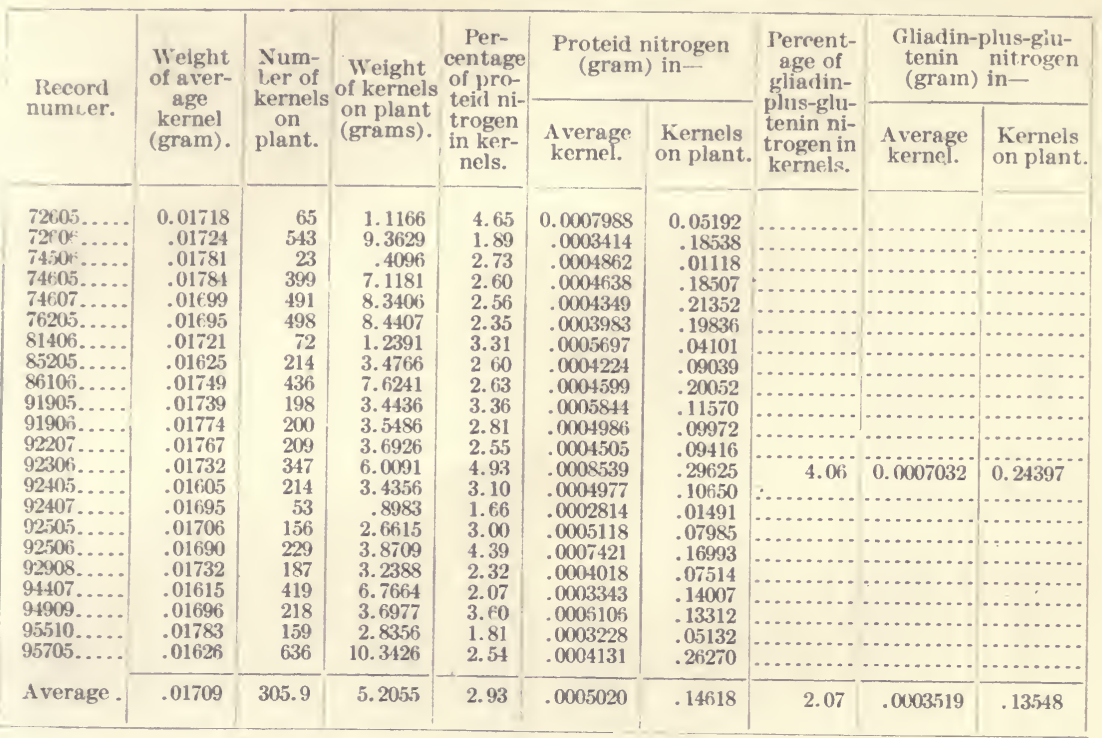

WEIGHT OF AVERAGE KERNEL, 0.018 TO 0.020 GRAM.

\begin{tabular}{|c|c|c|c|c|c|c|c|c|c|}
\hline $17305 \ldots .$. & 0.01984 & 183 & 3.6302 & 3.03 & $0.000 f 010$ & 0.10999 & & & \\
\hline $17408 \ldots$ & .01852 & 497 & 9.2038 & 2.18 & .0004037 & .20065 & & & \\
\hline $17409 \ldots$ & .01857 & 802 & 14.8957 & 2.75 & .0005108 & .40964 & & & \\
\hline $20710 \ldots .$. & .01974 & 867 & 17.1115 & 2.83 & .0005586 & .48428 & 2.00 & 0.0003948 & 0.34222 \\
\hline $21205 \ldots .$. & .01922 & 123 & 2.3642 & 3.16 & .0006074 & .07471 & & & $\begin{array}{l}0.01222 \\
\ldots . . . \ldots\end{array}$ \\
\hline $21206 . .$. & .01917 & 149 & 2.8564 & 5.23 & .0010026 & .14939 & & $\cdots$ & $\cdots$ \\
\hline $21207 \ldots$. & .01955 & 118 & 2.3066 & 2.96 & .0005766 & .06804 & & $\cdots$ & $\cdots$ \\
\hline $21306 . . .$. & .91837 & 226 & 4.1516 & 2.90 & .0005327 & .12039 & & & $\cdots$ \\
\hline $21711 \ldots$. & $.9 \mathrm{i} 968$ & 873 & 17.1820 & 2.71 & .0005331 & .465 t3 & & & \\
\hline & .01919 & 418 & 8.0214 & 2.73 & .0005238 & .21898 & 2.18 & .0004183 & .17487 \\
\hline $0 \ldots$ & $\begin{array}{l}.01982 \\
.01877\end{array}$ & $\begin{array}{r}52 \\
216\end{array}$ & 1. 0304 & 2.69 & .0005330 & .02772 & & $\cdots$ & \\
\hline $\begin{array}{l}21815 . . . . \\
21905 . \ldots\end{array}$ & $\begin{array}{l}.01877 \\
.01809\end{array}$ & $\begin{array}{l}216 \\
791\end{array}$ & $\begin{array}{r}4.0258 \\
4.311\end{array}$ & 4.04 & .0007582 & .16377 & 2.14 & .00 & \\
\hline $21907 \ldots$ & .01851 & $\begin{array}{l}791 \\
158\end{array}$ & $\begin{array}{r}14.3111 \\
2.9248\end{array}$ & 2.64 & 777 & .37781 & 2.18 & .00039 & .31 \\
\hline 21912 . & .01907 & 510 & $\begin{array}{l}2.9248 \\
9.7236\end{array}$ & $\begin{array}{l}3.35 \\
2.31\end{array}$ & $\begin{array}{l}.0006201 \\
.0004404\end{array}$ & $\begin{array}{r}.09798 \\
.22461\end{array}$ & 2.15 & 3980 & .06288 \\
\hline 22207 & .01940 & 169 & 3.2787 & 2.77 & .0005374 & $\begin{array}{l}.22401 \\
.09082\end{array}$ & 1.82 & 0003531 & .05967 \\
\hline 26905. & .01966 & 326 & 6.4102 & 2.76 & .000 .5427 & .17692 & 2.09 & .0004109 & .13398 \\
\hline 26906. & .01859 & 228 & 4.2376 & 2.71 & .0005037 & .11484 & 1.82 & .0003383 & .07712 \\
\hline $27005 \ldots .$. & .01895 & 866 & 16.4120 & 2.63 & .0004981 & .43164 & 1.90 & .0003600 & .31182 \\
\hline $27205 \ldots .$. & .01841 & 891 & 16.4061 & 2.41 & .0004437 & .39539 & 1.70 & .0003130 & .27890 \\
\hline $27306 \ldots$. & .01945 & 684 & 13. 3011 & 2.47 & .0004803 & .32853 & & & $\ldots$ \\
\hline $27307 \ldots .$. & .01847 & 167 & 3.0850 & 2.53 & .0004674 & .07805 & & - & $\ldots$ \\
\hline $27507 \ldots$. & .01833 & 75 & 1.3746 & 3.08 & .0005646 & .04234 & & & \\
\hline $28206 \ldots . .$. & .01996 & 219 & 4. 3698 & 3.07 & .0006126 & .13415 & 2.42 & 830 & .10575 \\
\hline $07 \ldots$. & .01822 & 69 & 1.2573 & 3.48 & .0006341 & .04375 & ........ & $\ldots$ & $\ldots$ \\
\hline $308 \ldots .$. & .01851 & 55 & 1.0183 & 3. 78 & .0006999 & .03849 & ....... & & \\
\hline $105 . . .$. & .01939 & 132 & 2.5601 & 2.91 & .0005647 & .07450 & 3.50 & 87 & .07450 \\
\hline $07 \ldots$. & .01919 & 318 & 6.1026 & 2.35 &.$\overline{0004510}$ & .14341 & 1.92 & .0004163 & .12643 \\
\hline $33405 . . .$. & .01930 & 421 & 8. 1268 & 2.03 & .0003919 & .16498 & . & ........ & $\ldots$ \\
\hline $33906 \ldots$ & .01921 & 119 & 2. 2862 & 2.81 & .0005399 & .06424 & & & \\
\hline $34205 \ldots \ldots$ & .01972 & 464 & 9.1498 & 2.73 & .0005383 & .24979 & & & \\
\hline $34206 \ldots .$. & .01968 & 81 & 1.5940 & 3.73 & .0007340 & .05946 & & & ... \\
\hline $208 \ldots$. & .01916 & 155 & 2. 9886 & 2.13 & .0004081 & .06366 & & & \\
\hline $05 \ldots \ldots$ & .01994 & $\begin{array}{l}207 \\
267\end{array}$ & 4. 1281 & 4.33 & .0008635 & .17875 & 2.44 & .0004865 & i \\
\hline & $\begin{array}{l}.01880 \\
.01987\end{array}$ & $\begin{array}{l}267 \\
309\end{array}$ & $\begin{array}{l}5.0200 \\
6.1394\end{array}$ & 3.88 & .0007295 & .19478 & & $\cdots$ & \\
\hline $5 \ldots \ldots$ & $\begin{array}{l}.01987 \\
.01972\end{array}$ & $\begin{array}{l}309 \\
461\end{array}$ & $\begin{array}{l}6.1394 \\
8.0905\end{array}$ & $\begin{array}{l}2.96 \\
2.64\end{array}$ & $\begin{array}{l}.0005881 \\
.0005327\end{array}$ & $\begin{array}{r}.18173 \\
.23998\end{array}$ & $\begin{array}{l}2.29 \\
1.26\end{array}$ & $\begin{array}{l}.0004550 \\
0002485\end{array}$ & $\begin{array}{l}.14060 \\
10194\end{array}$ \\
\hline $55 \ldots$. & .01808 & 139 & 2.5134 & $\begin{array}{l}2.64 \\
2.81\end{array}$ & $\begin{array}{l}.0005327 \\
.0005135\end{array}$ & .07138 & 1.23 & .0002224 & .03091 \\
\hline $6 \ldots$. & .01975 & 85 & 1.6799 & 2.89 & .0005712 & .04855 & $\ldots .$. & $\ldots$ & $\ldots$ \\
\hline .. & .01987 & 61 & 1.2124 & 5.85 & .0011627 & .07093 & & $\ldots$ & \\
\hline 3.... & .01913 & 159 & 3.0228 & 2.82 & .0005394 & .08522 & 1.73 & .0003309 & .05229 \\
\hline $\begin{array}{l}38706 \ldots . . . \\
40205\end{array}$ & $\begin{array}{r}.01988 \\
01871\end{array}$ & 365 & 7.2545 & 2.5 & & & 7 & & \\
\hline & 71 & 194 & 3.6 & 4.69 & .0008776 & 26 & & 5744 & 11 \\
\hline
\end{tabular}


TABLE 10.-Analyses of plants, arranged according to weight of average kernel. Crop of 1903-Continued.

WEIGHT OF AVERAGE KERNEL, 0.018 TO 0.020 GRAM-Continued.

\begin{tabular}{|c|c|c|c|c|c|c|c|c|c|}
\hline \multirow{2}{*}{$\begin{array}{l}\text { Record } \\
\text { number. }\end{array}$} & \multirow{2}{*}{$\begin{array}{l}\text { Weight } \\
\text { of aver- } \\
\text { age } \\
\text { kernel } \\
\text { (gram). }\end{array}$} & \multirow{2}{*}{$\begin{array}{l}\text { Num- } \\
\text { Ler of } \\
\text { kernels } \\
\text { on } \\
\text { plant. }\end{array}$} & \multirow{2}{*}{$\begin{array}{l}\text { Weight } \\
\text { of kernels } \\
\text { on plant } \\
\text { (grams). }\end{array}$} & \multirow{2}{*}{$\begin{array}{c}\text { Per- } \\
\text { centage } \\
\text { of pro- } \\
\text { teid ni- } \\
\text { trogen } \\
\text { in ker- } \\
\text { nels. }\end{array}$} & \multicolumn{2}{|c|}{$\begin{array}{l}\text { Proteid nitrogen } \\
\text { (gram) in- }\end{array}$} & \multirow{2}{*}{$\begin{array}{l}\text { Percent- } \\
\text { age of } \\
\text { gliadin- } \\
\text { plus-glu- } \\
\text { tenin ni- } \\
\text { trogen in } \\
\text { kernels. }\end{array}$} & \multicolumn{2}{|c|}{$\begin{array}{l}\text { Gliadin-plus-glu- } \\
\text { tenin nitrogen } \\
\text { (gram) in- }\end{array}$} \\
\hline & & & & & $\begin{array}{l}\text { Average } \\
\text { kernel. }\end{array}$ & $\begin{array}{l}\text { Kernels } \\
\text { on plant. }\end{array}$ & & $\begin{array}{l}\text { Average } \\
\text { kernel. }\end{array}$ & $\begin{array}{l}\text { Kernels } \\
\text { on plant. }\end{array}$ \\
\hline 42205. & 0.01967 & 94 & 1.8494 & 3.63 & 0.0007142 & 0.06713 & 2.73 & 0.0005370 & 0.05049 \\
\hline 42905 . & .01866 & 67 & 1.2499 & 3.17 & .0005447 & .03650 & $\ldots$ & .............. & . . . . . \\
\hline $44607 \ldots$ & .01806 & 101. & 1.8246 & 2.44 & .0004408 & 4452 & & & \\
\hline 45606 & .01834 & 220 & 4.0358 & 1.91 & .0003504 & .07708 & $\ldots$ & $\ldots$ & \\
\hline $46106 \ldots$. & .01964 & 82 & 1.6103 & 2.54 & .0004988 & .04090 & & & \\
\hline $48106 \ldots$ & .01919 & 608 & 11. 6655 & 2.38 & .0004567 & .27765 & 1.80 & .0003454 & .20997 \\
\hline 48508 & .01858 & 603 & 11.2008 & 2.76 & .0005127 & .30986 & $\ldots$. & ........... & $\ldots$. \\
\hline 49505 . & .01898 & 67 & 1.2716 & 3.24 & .0006149 & .04120 & .... & $\ldots$. & \\
\hline 50705 & .01986 & 30 & .5958 & 3.54 & .0007032 & .02109 & $\ldots$ & $\ldots$ & ... \\
\hline 51005. & .01804 & 862 & 15.5835 & 1.34 & .0002422 & .20881 & & $\ldots$ & \\
\hline 55007 & .01828 & 118 & 2. 1571 & 4. 21 & .0007696 & .09082 & 2.21 & .0004040 & .04767 \\
\hline 55 & .01846 & 944 & 17.4226 & 2.60 & .0004799 & .45299 & 1.58 & .0002917 & .27528 \\
\hline 55 & .01965 & 578 & 11.3592 & 2.56 & .0005031 & .29079 & 1.87 & .0003675 & .21241 \\
\hline 55 & 01931 & 214 & 4. 1323 & 2.18 & .0004210 & .09008 & & & \\
\hline 55 & .01949 & 504 & 9.8228 & 2.63 & .0005126 & .25834 & 2.07 & .0004034 & .20333 \\
\hline & .01866 & 644 & 12.0161 & 2.57 & .0004795 & .30881 & 2.09 & .0003900 & .25114 \\
\hline & .01959 & 333 & 6.5232 & 2.51 & .0004917 & .16373 & 1.85 & .0003624 & .12068 \\
\hline & .01829 & 509 & 9.3093 & 2.42 & .0004426 & .22529 & 1.95 & .0003566 & .18153 \\
\hline & .01975 & 168 & 3.3176 & 2.65 & .0005233 & .08792 & ..... & ........... & $\ldots \ldots$ \\
\hline & .01838 & 434 & 7.9772 & 2.86 & .0005257 & .22915 & $\ldots$ & $\ldots$ & $\ldots$ \\
\hline $07 \ldots$. & .01801 & 261 & 4. 7117 & 2.43 & .0004387 & .11445 & ...... & & \\
\hline $57406 \ldots$ & .01846 & 135 & 2.4923 & 2.75 & .0005077 & .06854 & 2.13 & .0003932 & .05309 \\
\hline 57407 . & .01968 & 762 & 14.9992 & 2.62 & .0005157 & .39297 & 1.86 & .0003660 & .27898 \\
\hline $57507 \ldots$ & .01946 & 359 & 6.9861 & 2.85 & .0005545 & .19905 & 1.55 & .0003016 & .10828 \\
\hline 57608 & .01968 & 438 & 8.6189 & 2.64 & .0005195 & .22756 & 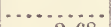 & & \\
\hline 57805 & .01814 & 270 & 4.8988 & 2.87 & .0005207 & .14060 & 2.68 . & .0004861 & .13 \\
\hline $58805 \ldots$ & .01999 & 1,158 & 23.1471 & 2.74 & $\because 0005464$ & .63422 & 2.11 & .0004218 & 39 \\
\hline 59605. & .01880 & 382 & 7.1828 & 2.12 & .0003986 & .15228 & . . . . . . . . & $\ldots$ & $\ldots$ \\
\hline 63505. & .01934 & 208 & 4. 0230 & 1.90 & .0003674 & .07644 & 10 & $\ldots$ & is \\
\hline 65306 & .01807 & 544 & 9.8298 & 2.41 & .0004282 & .23690 & 1.68 & .0003036 & 14 \\
\hline 65307 & .01878 & 373 & 7.0051 & 2.28 & .0004355 & .15971 & 1.81 & .0003399 & .12680 \\
\hline 66008 & .01814 & 174 & 3.1555 & 3.59 & .0006510 & .11328 & $\ldots \ldots$ & ............ & ............ \\
\hline 69305. & .01984 & 103 & 2.0430 & 4.42 & .0008767 & .09030 & $\ldots \ldots \ldots$ & . . . . . & $\ldots$ \\
\hline 69505. & .01847 & 255 & 4. 7116 & 2.29 & .0004231 & .10790 & .......... & $\ldots$ & $\ldots$ \\
\hline $72406 \ldots$ & .01929 & 430 & 8.2929 & 2.95 & .0005689 & .24464 & & & $\ldots$ \\
\hline 72607 & .01832 & 188 & 3.4442 & 5.59 & .0010241 & .19253 & 2.51 & .0004598 & .08645 \\
\hline 72806 & .01906 & 110 & 2.0970 & 3.01 & .0005738 & .06312 & .......... & ............ & ........... \\
\hline 74507. & .01869 & 493 & 9.2130 & 3.02 & .0005644 & .27823 & .......... & . .......... & .......... \\
\hline $81405 \ldots .$. & .01862 & 240 & 4.5737 & 2.62 & .0004879 & .11710 & & $\ldots$ & \\
\hline $81505 \ldots$. & .01940 & 146 & 2.8327 & 2.94 & .0005704 & .08328 & 2.65 & .0005141 & 507 \\
\hline $5 . . .$. & 01927 & 37 & .7130 & 2.32 & .0004471 & .01654 & $\ldots \ldots$. & . . . . . . . & $\ldots$ \\
\hline $84906 \ldots .$. & .01975 & 382 & 7.5438 & 3.43 & .0006773 & .25873 & .......... & & $\ldots$ \\
\hline$\ldots$ & .01811 & 293 & 5.3069 & 2.83 & .0005126 & .15019 & & & . \\
\hline 889 & .01814 & 546 & 9.9034 & 2.65 & .0004807 & .26245 & & & \\
\hline 922 & .01876 & 353 & 6.6206 & 2.72 & .0005102 & .18008 & & .. & $\ldots$ \\
\hline 9240 & .01827 & 207 & 3. 7820 & 2.97 . & .0005426 & .11233 & & & \\
\hline 9240 & .01814 & 315 & 5. 7131 & 2.30 & .0004171 & .13140 & & .. & 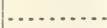 \\
\hline 925 & .01916 & 505 & 9.6779 & 2.58 & .0004944 & .24969 & $\ldots$ & $\ldots$ & . \\
\hline 92909 & .01916 & 529 & 10.1363 & 2.70 & .0005173 & .27367 & & ..... & $\cdots$ \\
\hline 94205. & .01893 & 64 & 1.2117 & 1.65 & .0003124 & .01999 & & ... & ... \\
\hline 942 & .01866 & 402 & 7.5006 & 2.78 & .0005187 & .20851 & & & ... \\
\hline 942 & .01909 & 718 & 13.7057 & 2.86 & .0005460 & .39199 & $\therefore$ & & $\ldots$ \\
\hline 94209 & .01895 & 190 & 3.6006 & 2.49 & .0004719 & .08965 & $\ldots$ & & $\ldots$ \\
\hline 94406. & .01923 & 549 & 10.5556 & 2.47 & .0004749 & .26073 & $\ldots$ & & $\ldots$ \\
\hline 94906 . & .01808 & 68.5 & 12.3862 & 3.41 & .0006166 & .42236 & $\cdots$ & & $\ldots$ \\
\hline $94907 \ldots$ & .01948 & 626 & 12. 1918 & 2.94 & .0005726 & .35814 & $\ldots$ & & \\
\hline 94908 . & .01894 & 125 & 2.3678 & 1.96 & .0003713 & .04641 & $\ldots$ & & $\ldots$ \\
\hline 95506. & .01852 & 597 & 11.0548 & 2.74 & .0005074 & .30291 & $\ldots$. & & $\ldots$. \\
\hline $95008 \ldots$. & .01954 & 740 & 14. 4617 & $2: 56$ & .0005003 & .37023 & $\ldots \ldots$ & . & $\ldots \ldots$ \\
\hline $95706 \ldots .$. & .01934 & 267. & 5.1629 & 2.73 & .0005279 & .14095 & $\ldots \ldots$ & & $\cdots$ \\
\hline Average. & .01901 & 349.6 & 6.6327 & 2.88 & .0005476 & .18039 & 2.08 & .0003979 & .15541 \\
\hline
\end{tabular}

WEIGHT OF AVERAGE KERNEL, 0.020 TO 0.022 GRAM.

\begin{tabular}{|c|c|c|c|c|c|c|c|c|c|}
\hline $17308 \ldots$ & 0.02012 & 61 & 1.2275 & 3.25 & 0.0006540 & 0.03994 & & & $\cdot \ldots$ \\
\hline $17405 \ldots$ & .02127 & 738 & 15.6996 & 2. 13 & .0004531 & .33441 & & & \\
\hline $20708 \ldots .$. & .02033 & 163 & 3.3138 & 2.78 & .0005652 & .09212 & 2.05 & 0.0004168 & 0.06793 \\
\hline $20708 . . .$. & .02024 & 122 & 2.4690 & 2.58 & .0005221 & .06399 & $331^{-}$ & …글. & $\cdots$ \\
\hline $20709 \ldots$. & .02023 & 258 & 5.3229 & 3.05 & .0005292 & .16235 & 2.31 & .0004766 & .12296 \\
\hline $20805 . . .$. & .02157 & $€ 97$ & 14.6942 & 3.32 & .000 .1999 & .48784 & 2.26 & .0004875 & .33208 \\
\hline
\end{tabular}


TABLE 10.-Analyses of plants, arranged according to weight of average kernel. Crop of 1903 - Continued.

WEIGHT OF AVERAGE KERNEL, 0.020 TO 0.022 GRAM-Continued.

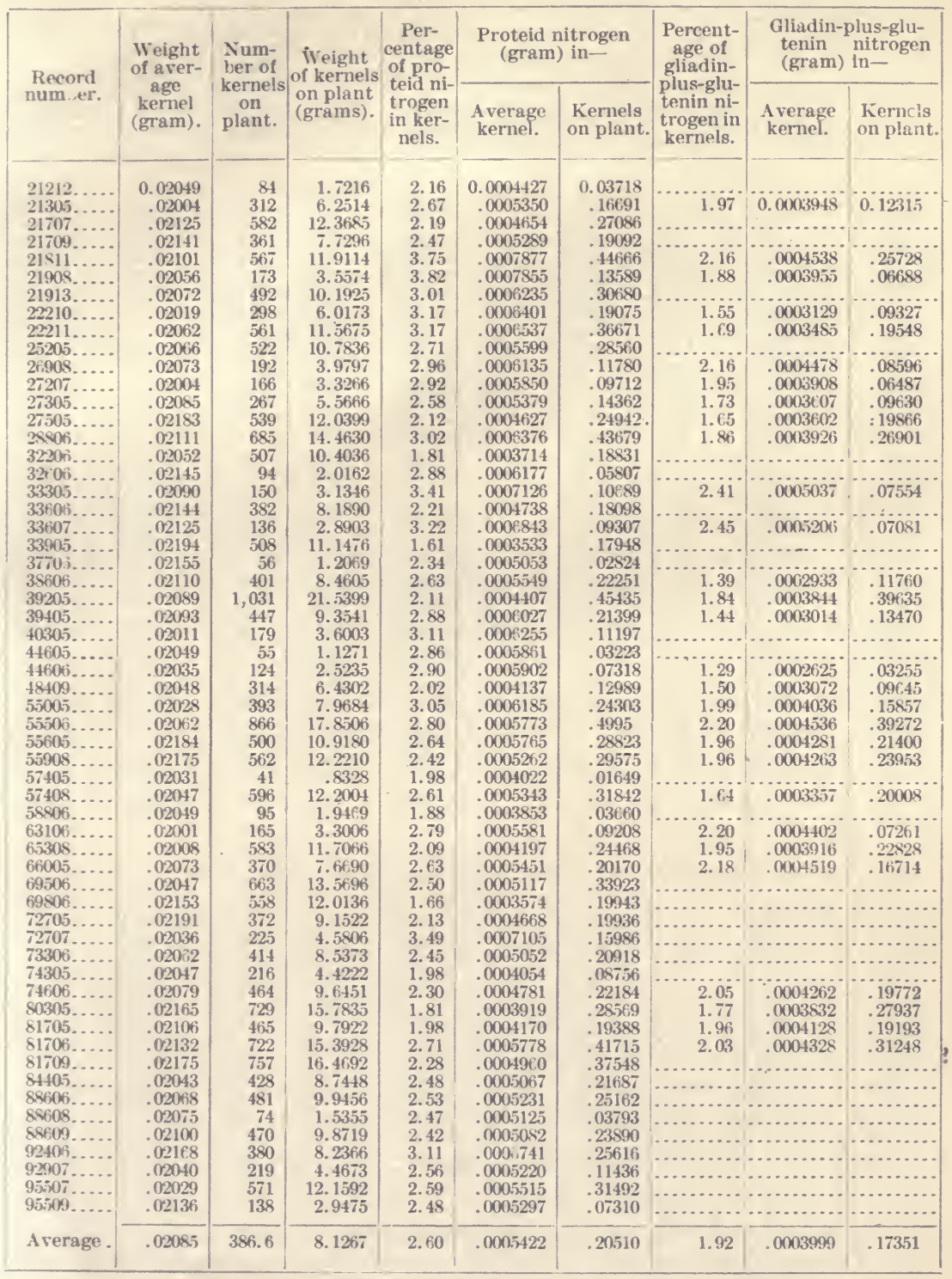

WEIGHT OF AVRRAGE KERNEL, 0.022 TO 0.024 GRAM.

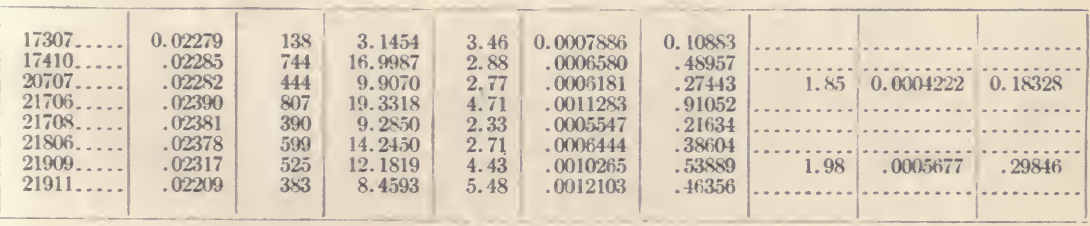


TABLE 10.-Analyses of plants, arranged according to weight of average kernel. Crop of 1903-Continued.

WEIGHT OF AVERAGE KERNEL, J.022 TQ 0.024 GRAM-Continued.

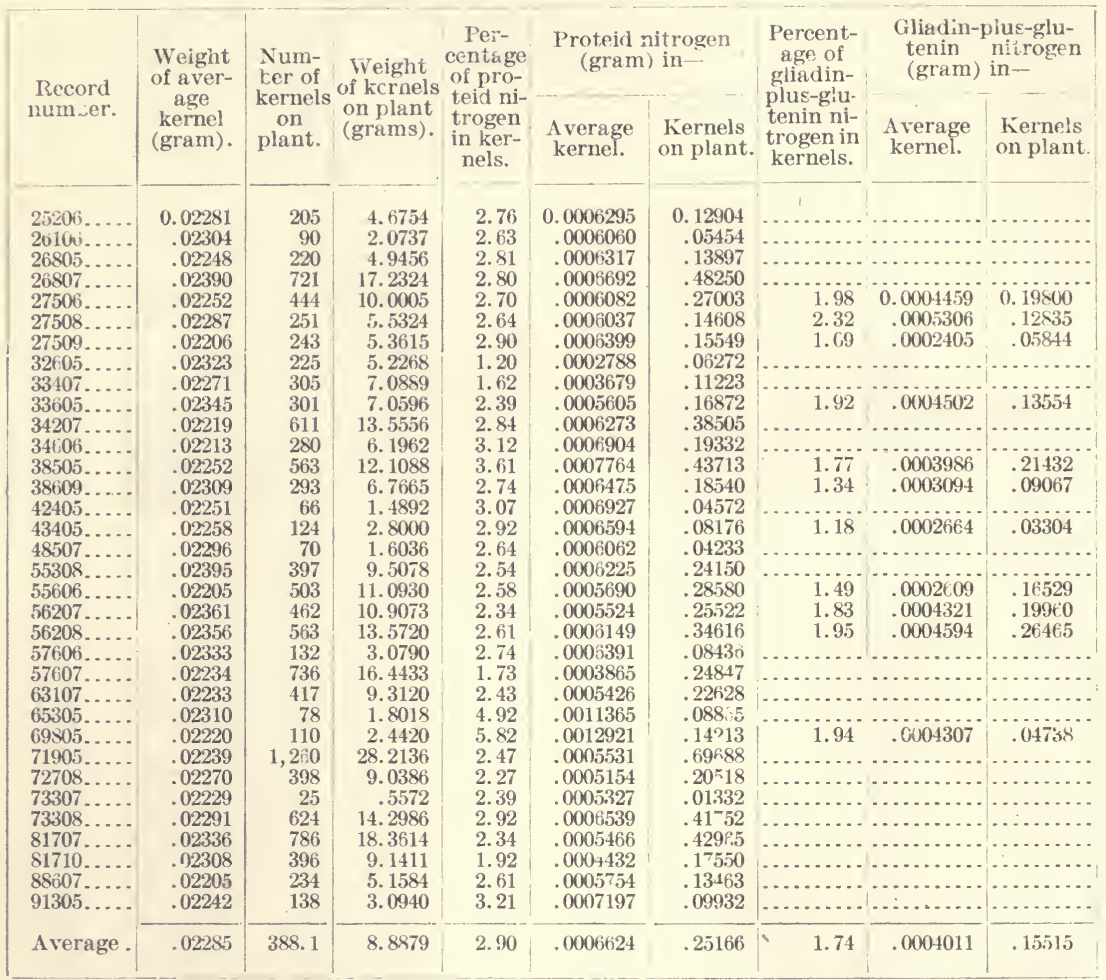

WEIGHT OF AVERAGE KERNEL, 0.024 TO 0.026 GRAM.

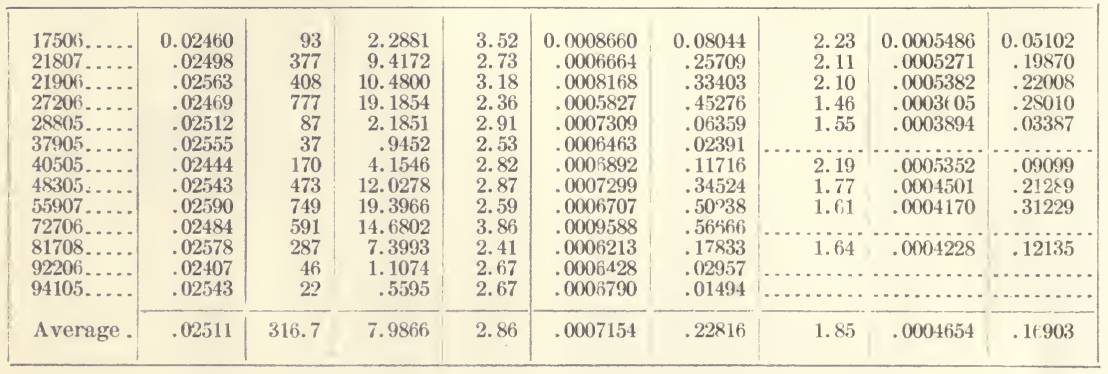

WEIGIT OF AVERAGE KERNEL, 0.026 GRAM AND OVER.

\begin{tabular}{|c|c|c|c|c|c|c|c|c|c|}
\hline $39506 \ldots$. & .02869 & 67 & 1.9218 & 2.93 & .0008404 & .05631 & 2.06 & 0.0005915 & 0.03959 \\
\hline $5.5608 \ldots$. & .02699 & 837 & 22.5848 & 2.31 & .0006236 & .52194 & 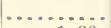 & ............ & $\ldots \ldots$ \\
\hline $55909 \ldots \ldots$ & .03050 & 302 & 9.2120 & 2.30 & $.00 u^{7} 016$ & .21187 & 1.66 & $.000 \pi 0 \leqq 3$ & .15292 \\
\hline $57508 \ldots$. & .03177 & 380 & 12.0728 & 2.21 & .0007021 & .26680 & 2.05 & .0006513 & .24750 \\
\hline $58505 \ldots . .$. & .02730 & 273 & 7.4516 & 2.95 & .0008052 & .21982 & ......... & $\ldots \ldots \ldots \ldots$ & $\ldots \ldots \ldots$ \\
\hline Average. & .02988 & 240.3 & 7.2425 & 2.81 & .0008449 & .18126 & 1.92 & .0005829 & .14667 \\
\hline
\end{tabular}


TABLE 11.-Summary of analyses of plants, arranged according to weight of average kernel. Crop of 1903.

\begin{tabular}{|c|c|c|c|c|c|c|c|c|c|c|}
\hline \multirow[b]{2}{*}{$\begin{array}{c}\text { Range of } \\
\text { weights of } \\
\text { a verage kernel } \\
\text { (gram). }\end{array}$} & \multirow[b]{2}{*}{$\begin{array}{l}\text { Num- } \\
\text { ber of } \\
\text { analy- } \\
\text { ses. }\end{array}$} & \multirow[b]{2}{*}{$\begin{array}{l}\text { Weight } \\
\text { of aver- } \\
\text { age ker- } \\
\text { nel } \\
\text { (gram). }\end{array}$} & \multirow[b]{2}{*}{$\begin{array}{l}\text { Num- } \\
\text { ber cf } \\
\text { kernels. }\end{array}$} & \multirow[b]{2}{*}{$\begin{array}{l}\text { Weight } \\
\text { of ker- } \\
\text { nels } \\
\text { (grams). }\end{array}$} & \multirow{2}{*}{$\begin{array}{l}\text { Per- } \\
\text { cent- } \\
\text { age of } \\
\text { pro- } \\
\text { teid ni- } \\
\text { trogen } \\
\text { in ker- } \\
\text { nels. }\end{array}$} & \multicolumn{2}{|c|}{$\begin{array}{l}\text { Proteid nitrogen } \\
\text { (gram) in- }\end{array}$} & \multirow{2}{*}{\begin{tabular}{|l} 
Per \\
cent- \\
age of \\
glia- \\
din- \\
plus- \\
glu- \\
tenin \\
nitro- \\
gen in \\
ker- \\
nels.
\end{tabular}} & \multicolumn{2}{|c|}{$\begin{array}{l}\text { G lia din-p lus- } \\
\text { glutenin nitro- } \\
\text { gen(gram)in- }\end{array}$} \\
\hline & & & & & & $\begin{array}{l}\text { Average } \\
\text { kernel. }\end{array}$ & $\begin{array}{l}\text { Ker- } \\
\text { nels. }\end{array}$ & & $\begin{array}{l}\text { Average } \\
\text { kernel. }\end{array}$ & $\begin{array}{l}\text { Ker- } \\
\text { nels. }\end{array}$ \\
\hline 0.000 to $0.010 \ldots$ & 4 & 0.00915 & 219 & 2.0334 & 2.76 & 0.0002525 & 0.05618 & 1.97 & 0.0001877 & 0.05312 \\
\hline 0.010 to 0.012 . & 6 & .01118 & 179 & 2.0187 & 2.98 & .0003326 & .06276 & 2.69 & $.000296 \mathrm{~S}$ & .06752 \\
\hline 0.012 to 0.014. & 19 & .01323 & 155.7 & 2.0510 & 3.12 & .0004120 & .06687 & 1.98 & .0002641 & .07499 \\
\hline 0.014 to $0.016 \ldots$ & 27 & .01516 & 232 & 3.5480 & 3.00 & .0004555 & .10619 & 1. 76 & .0002805 & .09320 \\
\hline 0.016 to $0.018 \ldots$ & 69 & .01709 & 305.9 & 5.2055 & 2.93 & .0005020 & .14618 & 2.07 & .0003519 & .13548 \\
\hline 0.018 to $0.020 . .$. & 103 & .01901 & 349.6 & 6.6327 & 2.88 & .0005476 & .18039 & 2.08 & .0003979 & .15541 \\
\hline 0.020 to $0.022 . .$. & 64 & .02085 & 386.6 & 8.1257 & 2.60 & $.000 \pi 422$ & .20510 & 1.92 & .0003999 & .17351 \\
\hline 0.022 to $0.024 \ldots$ & $\$ 2$ & .02285 & 388.1 & 8.8879 & 2.90 & .0006624 & .25166 & 1.74 & .0004011 & .15515 \\
\hline 0.024 to $0.026 \ldots$ & 13 & .02511 & 316.7 & ‥9866 & 2.86 & .0007154 & $.22 \$ 16$ & 1.85 & .0004654 & .16903 \\
\hline 0.026 and over.. & 9 & .02988 & 240.3 & 7.2425 & 2.81 & .0008449 & .18126 & 1.92 & .000 .5829 & $.1466 \pi$ \\
\hline
\end{tabular}

With an increase in the weight of the kernel, as shown by this table, there is an irregular increase in the number of kernels on the plant up to a point somewhat beyond the kernel of average weight, after which there is a decrease. The weight of the kernels on the plant seems to follow the same rule. The percentage of proteid nitrogen in the kernels decreases, in general, with the weight of the average kernel, while the number of grams of proteid nitrogen in the average kernel increases steadily. The grams of proteid nitrogen in all the kernels on the plant increase up to the same point as do the number of kernels on the plant, and then decrease.

Table 12 shows the summary of the analyses of the crop of 1903, arlanged according to the grams of proteid nitrogen in the average kernel. All plants laving less than 0.0003 gram of proteid nitrogen form the first class, and the following classes increase with each 0.0001 gram of proteid nitrogen.

It is difficult to trace any relation between the grams of proteid nitrogen in the avelage kernal and the number of kernels on the plant, or the weight of the kernels on the plant. The weight of the average kernel increases directly with the grams of proteid nitrogen in the kernel. The percentage of proteid nitrogen increases regularly with an increase in the grams of proteid nitrogen in the average kernel. The grams of proteid nitrogen in all the kernels on the plant show no definite relation to the grams of proteid nitrogen in the average kernel.

It becomes evident from these results that selection of large, heary kernels for seed would result in discarding the immature and unsound kernels, but that there would also be discarded many sound kernels, which, although small and of low specific graviry, would contain a high percentage of proteids. 
Another effect of such selection, as indicated by the foregoing results, would be to increase the yield of grain from each plant when grown under the conditions that obtained in these experiments. What the effect would be upon the yield under ordinary field conditions these experiments do not indicate.

On the other hand, selection based upon percentage of proteid nitrogen alone would not result in securing plants of greatest yield when raised under these conditions. "It would, moreover, not result in obtaining plants producing the greatest amount of proteid nitrogen, nor even of kernels containing the largest quantity of proteid nitrogen.

TABLE 12.-Summary of analyses of plants, arranged according to grams of proteid nitrogen in average kernel. Crop of 1903.

\begin{tabular}{|c|c|c|c|c|c|c|c|}
\hline \multirow{2}{*}{$\begin{array}{l}\text { Range of proteid nitrogen in } \\
\text { average kernel (gram). }\end{array}$} & \multirow{2}{*}{$\begin{array}{c}\text { Proteid } \\
\text { nitrogen } \\
\text { in average } \\
\text { kernel } \\
\text { (gram). }\end{array}$} & \multirow{2}{*}{$\begin{array}{l}\text { Num- } \\
\text { ber of } \\
\text { analy- } \\
\text { ses. }\end{array}$} & \multirow{2}{*}{$\begin{array}{c}\text { Number } \\
\text { of ker- } \\
\text { nels on } \\
\text { plant. }\end{array}$} & \multicolumn{2}{|c|}{$\begin{array}{c}\text { Weight (in grams) } \\
\text { of- }\end{array}$} & \multirow{2}{*}{$\begin{array}{l}\text { Percent- } \\
\text { age of } \\
\text { proteid } \\
\text { nitrogen } \\
\text { in ker- } \\
\text { nels. }\end{array}$} & \multirow{2}{*}{$\begin{array}{l}\text { Proteid } \\
\text { nitrogen } \\
\text { in ker- } \\
\text { nels on } \\
\text { plant } \\
\text { (gram). }\end{array}$} \\
\hline & & & & $\begin{array}{l}\text { Kernels } \\
\text { on plant. }\end{array}$ & $\begin{array}{l}\text { Average } \\
\text { kernel. }\end{array}$ & & \\
\hline Below 0.00030 . & 0.0002509 & 14 & 257.9 & 3.9190 & 0.01364 & 1.96 & 0.06531 \\
\hline 0.00030 to $0.00040 \ldots . . . . .$. & .0003602 & 42 & 266.7 & 4. 6742 & .01628 & 2.31 & .09644 \\
\hline 0.00040 to $0.00050 \ldots \ldots$. & .0004537 & 80 & 409.2 & 7.5309 & .01811 & 2.54 & .18644 \\
\hline 0.00050 to $0.00060 \ldots$ & .0005406 & 116 & 341.5 & 6.7159 & .01908 & 2.86 & .18440 \\
\hline 0.00060 to $0.00070 \ldots$ & .0006409 & 59 & 310. & 6.7257 & .02137 & 3.07 & .19805 \\
\hline 0.00070 to 0.00080 & .0007430 & 24 & 20 & 4.5158 & .02110 & 3.66 & .15318 \\
\hline 0.00080 to $0.00090 \ldots$ & .0008538 & 9 & 189 & 4. 2480 & .02334 & 3.79 & .15944 \\
\hline 0.00090 to $0.00100 \ldots \ldots . . . . .$. & .0009588 & 1 & 591.0 & 14.6802 & .02484 & 3.86 & .56666 \\
\hline 0.00100 and over.......... & .0011578 & 11 & 244.9 & 6.6082 & .02875 & 4.62 & .27980 \\
\hline
\end{tabular}

It will be shown later that the determination of gliadin-plus-glutenin nitrogen is a safer guide to the bread-making value of wheat than is a determination of proteid nitrogen, but whether selection should be based upon the percentage of nitrogen or the total production of nitrogen by the plant, or upon the amount contained in the average kernel, is a question that can not be solved except by trial under field conditions.

Some results of experiments with light and with heavy seed conducted on large field plots for several years may throw some light on this subject, and are given herewith.

\section{YIELD OF NITROGEN PER ACRE.}

. It is important to know whether the absolute amount of nitrogen per acre of grain raised is greater in light or in heavy wheat.

If the absolute amount of nitrogen per acre is less in light than in heavy wheat the supposition would be justifiable that the kernels were immature or had been prematurely checked in their development. On the other hand, if the amount of nitrogen per acre is greater in the light wheat it would be reasonable to suppose that, as both had been raised under the same conditions, the light wheat had, in part at least, come from plants that possessed greater ability to acquire and elaborate nitrogenous material. 
To afford information on this point analyses were made of crops grown from light and from heavy seed. Records of the yields of the plots were kept in each case so that the actual amount of proteid nitrogen contained in an acre of each kind of wheat can be calculated. The number of grams of proteid nitrogen in 1,000 kernels of each seed and crop sample is also stated. The first samples separated, Nos. 78 and 79 of the Turkish Red varieiy and 80 and 81 of the Big Frame variety, were taken from seed that had never before been treated in this way. When planted they produced the crops indicated in Table 13 by $78 \mathrm{~b}, 79 \mathrm{~b}, 80 \mathrm{~b}$, and $81 \mathrm{~b}$, respectively. Each of these crops was then separated into two portions, of which the light portion of the light wheat was retained for analyzing and planting, and the heavy portion of the heavy wheat likewise retained. Thus No. 383 is the light portion of No. $78 \mathrm{~b}$, and No. 384 is the heavy portion of No. 79 b.

The accuracy of the records of relative yields of light and heavy seed harvested in 1902 being open to suspicion, samples of the same seed were-sown again in the autumn of 1902 and harvested in 1903. The results from this test are stated at the bottom of the table under the heading "'Check experiment."

These experiments are to be understood as duplicating those of 1902 , which, as regards the relative yield of light and heavy wheat, should be accurate, although tried in 1903. The difference between this check experiment and the regular one of 1903 is that in the check experiment the seed of the crop of 1901 was used, while in the regular experiment in 1903 the seed of the crop of 1902 was used.

TABLE 13.-Crops grown from light and from heavy seed for four years.

SEED

\begin{tabular}{|c|c|c|c|c|c|c|c|}
\hline \multirow{2}{*}{$\begin{array}{l}\text { Farm } \\
\text { num- } \\
\text { ber. }\end{array}$} & \multirow[b]{2}{*}{ Variety. } & \multicolumn{3}{|c|}{ Percentage of- } & \multirow{2}{*}{$\begin{array}{l}\text { Weight of } \\
1,000 \text { ker- } \\
\text { nels. } \\
\text { (grams). }\end{array}$} & \multirow{2}{*}{$\begin{array}{l}\text { Proteid } \\
\text { nitrogen } \\
\text { in } 1,000 \\
\text { kernels } \\
\text { (gram). }\end{array}$} & \multirow[b]{2}{*}{$\begin{array}{l}\text { Relative } \\
\text { weight. }\end{array}$} \\
\hline & & $\begin{array}{c}\text { Total } \\
\text { nitrogen. }\end{array}$ & $\begin{array}{c}\text { Proteid } \\
\text { nitrogen. }\end{array}$ & $\begin{array}{c}\text { Non- } \\
\text { proteid } \\
\text { nitrogen. }\end{array}$ & & & \\
\hline $\begin{array}{r}78 \\
79 \\
80 \\
81 \\
383 \\
384 \\
385 \\
386\end{array}$ & 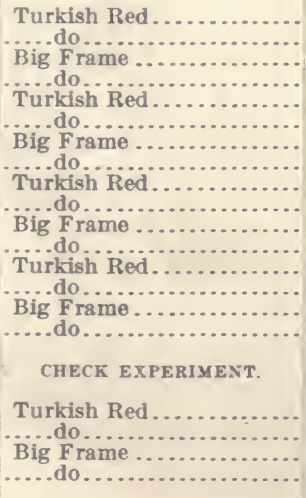 & $\begin{array}{r}\ldots .45 \\
2.45 \\
2.20 \\
3.12 \\
3.02 \\
3.13 \\
2.95 \\
\ldots \ldots \ldots \\
\ldots \ldots \ldots \\
\ldots \ldots \\
3.33 \\
3.06 \\
2.88 \\
\ldots \ldots\end{array}$ & $\begin{array}{r}\ldots \ldots \\
2.00 \\
1.96 \\
3.10 \\
2.93 \\
2.82 \\
2.65 \\
\ldots \ldots \ldots \\
\ldots \ldots \\
\ldots \ldots \\
\ldots \ldots \\
2.87 \\
2.86 \\
2.63 \\
\ldots \ldots .\end{array}$ & $\begin{array}{r}\ldots \ldots \\
0.45 \\
.24 \\
.02 \\
.09 \\
.31 \\
.30 \\
.30 \\
\ldots \ldots \ldots \\
\ldots \ldots \ldots \\
\ldots \ldots \ldots \\
\ldots .46 \\
.20 \\
.25 \\
\ldots \ldots \ldots\end{array}$ & 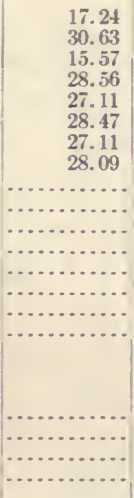 & 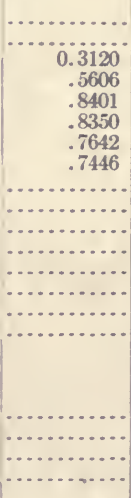 & $\begin{array}{l}\text { Light. } \\
\text { Heavy. } \\
\text { Light. } \\
\text { Heavy. } \\
\text { Light. } \\
\text { Heavy. } \\
\text { Light. } \\
\text { Heavy. } \\
\text { Light. } \\
\text { Heavy. } \\
\text { Light. } \\
\text { Heavy. } \\
\text { Light. } \\
\text { Heavy. } \\
\text { Light. } \\
\text { Heavy. }\end{array}$ \\
\hline
\end{tabular}


TABLE 13.-Crops grown from light and from heavy seed for four years - Continued.

CROP.

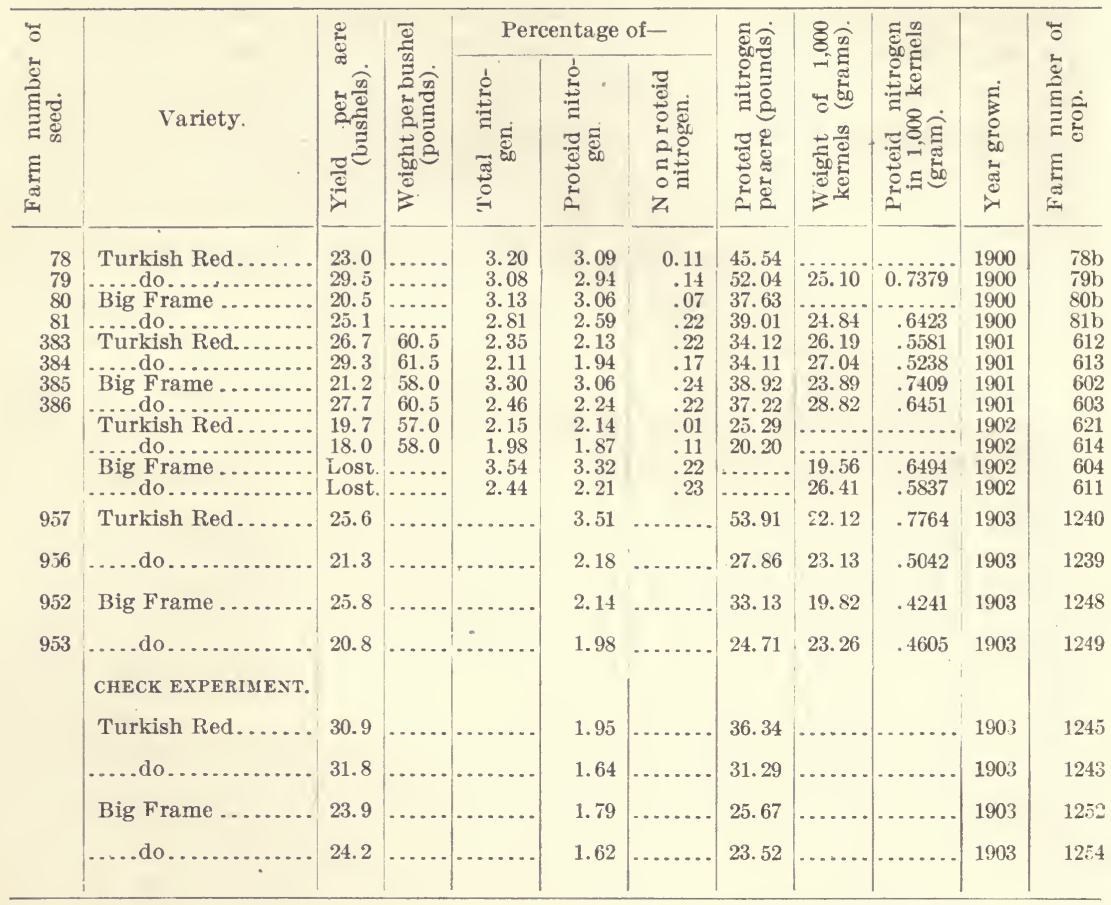

Comparing the analyses of the light and heavy seed in this table with those in the preceding tables, it will be noticed that the total and proteid nitrogen are both uniformly higher in the light seed. The nonproteid nitrogen is not so uniform as in the previous analyses, but the general tendency is the same.

In the crop the high total and proteid nitrogen of the light seed is uniformly transmitted. There is no uniformity in the nonproteid nitrogen. As was to be expected, the heavy seed produced in the first two years the largest yields per acre. The quality of light or heavy weight as indicated in the resulting crop by weight of grain per bushel gave some indication of being transmitted. In 1900 there was an absence of data on the subject, but in 1901 the heavy seed in each case produced grain having a greater weight per bushel than did the light seed.

Turning to the column showing the absolute amount of proteid nitrogen produced per acre, it is very apparent that the heavy seed produced in 1900 considerably larger amounts of proteid nitrogen per acre than did the light seed, but in 1901 the difference was very slightly in favor of the light wheat, which advantage continued with the light wheat during the remaining years. 
It would seem from these results that the quality of lightness, with its correlated qualities of high total and proteid nitrogen, is hereditary. The question then arises, Why should the light wheat accumulate more nitrogen per acre than the heavy wheat after the first generation?

A possible explanation for this is that the light seed from the first generation contained kernels whose lightness was due in some cases to immaturity, and in other cases to the individual peculiarity of the plant on which they grew. The latter class transmitted this peculiarity in the crop, while the former became less conspicuous with each generation, on account of the lesser vitality and productiveness of the immature seed.

A peculiar feature of these results is found in the fact that the yield of grain from the light seed approaches each succeeding year more nearly in quantity to that obtained from the heavy seed until, in 1903, it becomes greater. These two qualities of seed were raised on plots side by side, and every precaution was taken to obtain an accurate estimate of the yield of each. While it is probable that the results for 1903 are misleading, it is certainly significant that so little difference in yield exists after three years' selection in this way. Instead of the difference between the light and heary seed becoming greater each year it is without doubt becoming less.

In considering the relative yields of the light and heavy wheat, it must be borne in mind that the seeding was done with a drill set to deliver $1 \frac{1}{2}$ bushels per acre of ordinary seed wheat. The result would be to deposit a larger number of kernels of light seed per acre than of heavy seed. In a season like that of 1903, when the rainfall was large and the weather moderately cool until harvest, there might be an advantage resulting from the thicker seeding, which may account for the greater yield from the light seed in that year. It is possible that the same cause may have operated in other years to increase the yields from the light seed, but it is not likely that it produced a very marked effect, because the seeding was a large one for Nebraska, and, the wheat being sown in the early fall, there was abundant opportunity for it to stool, and thus equalize the stand. It has never been observed that there was any difference-between the plots in this respect.

Taking, together, the results of 1902, which show a decrease in the weight of the kernels on a single head as the content of proteid nitrogen increases, the results of 1903 , which show a slight decrease in the weight of the kernels from the plant, accompanying an increase in the percentage of proteid nitrogen, and the yields of the light and heavy seed for the four years beginning with 1900, there would appear to be a slight decrease in yield of grain, accompanying an increase in the percentage of proteid nitrogen. This loss in yield is 
not sufficient to counteract the increase in nitrogen, and the result is to increase the production of proteids per acre.

Viewed in the light of these various experiments, the selection of large, heavy wheat kernels for seed does not appear to be altogether unobjectionable, as in this case it resulted in a decreased production of proteids per acre, without a compensating increase in the yield of grain, when continued for a number of years. On the other hand, the selection of the small, light seed is hardly to be recommended. In fact, selection based upon kernel size or weight is not a satisfactory method for permanently improving wheat. The individual plant should be taken as the basis for selection, and very large numbers should be handled. The figures in Table 8 show what great opportunity there is for securing not only kernels of high nitrogen content, but also plants giving at the same time an increased yield of grain and abundant production of proteids. If the average nitrogen content and yield of grain by plants be observed in this table, it will be seen that numerous plants may be selected that have not only a nitrogen content above the average, but also a greater yield of grain. While, therefore, it is probable that improvement in yield of grain can not be effected so rapidly where it is combined with improvement in nitrogen content as if the latter were neglected, yet present yields of wheat in Nebraska can be increased at the same time that the production of proteids is augmented.

\section{METHOD FOR SELECTION TO INCREASE THE QUANTITY OF PROTEIDS IN THE KERNEL.}

The following tables show the results of analyses of a total of forty-eight spikes of wheat. In the case of each spike one row of spikelets, for instance, row No. 1, was analyzed, and the other row of spikelets, which would then be row No. 2, was analyzed separately. In the case of the set of spikes forming Table 14 the total organic nitrogen was determined in both lots, and in the set comprised by Table 15 the proteid nitrogen was deternnined. The last column shows the difference between the nitrogen content of the two rows of kernels. 
TABLE 14.-Analyses of twenty-five spikes of wheat, showing their total organic nitrogen.

\begin{tabular}{|c|c|c|c|c|c|c|c|}
\hline \multirow{2}{*}{ Number of spike. } & \multicolumn{3}{|c|}{$\begin{array}{l}\text { Percentage of total organic } \\
\text { nitrogen. }\end{array}$} & \multirow{2}{*}{ Number of spike. } & \multicolumn{3}{|c|}{$\begin{array}{l}\text { Percentage of total organic } \\
\text { nitrogen. }\end{array}$} \\
\hline & Row 1. & Row 2. & $\begin{array}{l}\text { Ditier- } \\
\text { ence. }\end{array}$ & & Row 1. & Row 2. & $\begin{array}{l}\text { Differ- } \\
\text { ence. }\end{array}$ \\
\hline 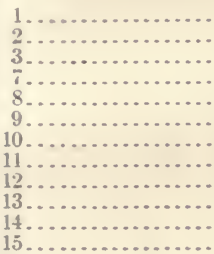 & $\begin{array}{l}3.14 \\
2.97 \\
2.89 \\
2.99 \\
2.89 \\
2.82 \\
2.50 \\
3.13 \\
3.11 \\
2.76 \\
2.85 \\
3.26\end{array}$ & $\begin{array}{l}3.32 \\
3.15 \\
2.99 \\
3.21 \\
2.82 \\
2.81 \\
2.76 \\
3.11 \\
3.18 \\
2.80 \\
2.79 \\
3.07\end{array}$ & $\begin{array}{l}0.18 \\
.15 \\
.10 \\
.22 \\
.07 \\
.01 \\
.26 \\
.02 \\
.07 \\
.04 \\
.06 \\
.19\end{array}$ & 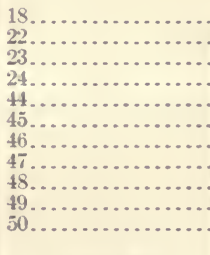 & $\begin{array}{l}2.83 \\
2.78 \\
2.94 \\
2.98 \\
3.00 \\
2.84 \\
3.03 \\
2.65 \\
2.62 \\
3.02 \\
3.02\end{array}$ & $\begin{array}{l}2.79 \\
2.76 \\
3.03 \\
2.89 \\
3.08 \\
2.67 \\
2.90 \\
2.79 \\
2.84 \\
3.18 \\
2.80\end{array}$ & $\begin{array}{l}0.04 \\
.02 \\
.09 \\
.09 \\
.08 \\
.17 \\
.13 \\
.14 \\
.22 \\
.16 \\
.22\end{array}$ \\
\hline $16 \ldots \ldots \ldots \ldots \ldots \ldots \ldots$ & $\begin{array}{l}2.94 \\
3.45\end{array}$ & $\begin{array}{l}3.07 \\
3.67\end{array}$ & $\begin{array}{l}.13 \\
.22\end{array}$ & A & & & .12 \\
\hline
\end{tabular}

TABLE 15.-Analyses of twenty-three spikes of wheat, showing their percentage of proteid nitrogen.

\begin{tabular}{|c|c|c|c|c|c|c|c|}
\hline \multirow{2}{*}{ Number of spike. } & \multicolumn{3}{|c|}{$\begin{array}{l}\text { Percentage of proteid } \\
\text { nitrogen. }\end{array}$} & \multirow{2}{*}{ Number of spike. } & \multicolumn{3}{|c|}{$\begin{array}{l}\text { Percentage of proteid } \\
\text { nitrogen. }\end{array}$} \\
\hline & Row 1. & Row 2. & $\begin{array}{l}\text { Differ- } \\
\text { ence. }\end{array}$ & & Row 1. & Row 2. & $\begin{array}{l}\text { Differ- } \\
\text { ence. }\end{array}$ \\
\hline $\begin{array}{c}4 \ldots \\
5 \ldots \\
20 \ldots \\
21 \ldots \\
25 \ldots \\
26 \ldots \\
27 \ldots \\
28 \ldots \\
30 \ldots \\
31\end{array}$ & $\begin{array}{l}2.90 \\
2.97 \\
2.68 \\
2.54 \\
2.42 \\
2.42 \\
3.01 \\
2.35 \\
2.72 \\
2.49 \\
2.92\end{array}$ & $\begin{array}{l}3.12 \\
2.86 \\
2.79 \\
2.76 \\
2.53 \\
2.50 \\
2.91 \\
2.71 \\
2.75 \\
2.44 \\
3.09\end{array}$ & $\begin{array}{l}0.22 \\
.11 \\
.11 \\
.22 \\
.11 \\
.08 \\
.10 \\
.03 \\
.03 \\
.17\end{array}$ & 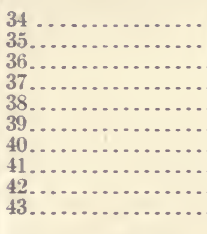 & $\begin{array}{l}2.86 \\
2.33 \\
2.88 \\
2.43 \\
3.15 \\
3.46 \\
2.45 \\
2.73 \\
3.42 \\
2.47 \\
\end{array}$ & $\begin{array}{l}3.02 \\
2.52 \\
2.85 \\
2.45 \\
3.14 \\
3.34 \\
2.59 \\
2.68 \\
3.61 \\
2.54 \\
\end{array}$ & $\begin{array}{l}0.16 \\
.19 \\
.03 \\
.02 \\
.01 \\
.12 \\
.14 \\
.05 \\
.19 \\
.07 \\
\end{array}$ \\
\hline $32 \ldots \ldots \ldots$ & $\begin{array}{l}2.60 \\
3.41\end{array}$ & $\begin{array}{l}2.48 \\
3.37\end{array}$ & $\begin{array}{l}.12 \\
.04\end{array}$ & A rerage.... & 2.77 & 2.82 & .11 \\
\hline
\end{tabular}

It will readily be seen that the analyses of the rows agree very closely, the extreme difference being 0.22 per cent, and the average difference being 0.12 per cent, in the total nitrogen. If, therefore, one row of spikelets were to be used for seed and the other were analyzed, it is quite evident that a very accurate estimate of the nitrogen content of the kernels used for seed would be obtained. In the determination of proteid nitrogen there is an extreme difference of 0.36 per cent in one case, but in the main the differences are small. As will be shown later, the variation in the proteid nitrogen content of individual plants is so great that even this maximum difference would cause no confusion when selecting plants for reproduction.

It is very desirable to have for analysis a larger sample than can be obtained from one spike. It has therefore been attempted to ascertain whether a sample consisting of one-half the whole number of spikes on a plant would afford a fair estimate of the composition of the other kernels on the remainder of the spikes. The plants whose spikes were analyzed were grown in hills 5 inches apart 
each way, with one seed in each hill. Each plant was harvested separately and the spikes from each placed in a separate envelope. The following table gives the results, lot 1 in each case being composed of the kernels from one-half the number of spikes on a plant, and lot 2 of kernels from the remaining spikes.

TABLE 16.-Analyses of twenty-one plants, showing total nitrogen and proteid nitrogen.

\begin{tabular}{|c|c|c|c|c|c|c|}
\hline \multirow{2}{*}{ Number of plant. } & \multicolumn{3}{|c|}{ Percentage of total nitrogen. } & \multicolumn{3}{|c|}{$\begin{array}{c}\text { Percentage of proteid } \\
\text { nitrogen. }\end{array}$} \\
\hline & Lot 1. & Lot 2. & $\begin{array}{l}\text { Differ- } \\
\text { ence. }\end{array}$ & Lot 1. & Lot 2. & $\begin{array}{l}\text { Differ- } \\
\text { ence. }\end{array}$ \\
\hline $1 .$. & 2.65 & 2.91 & 0.26 & 2.51 & 2.69 & 0.18 \\
\hline $2 \ldots$ & 3.01 & 3.02 & .01 & 2.77 & 2.76 & .01 \\
\hline $3 \ldots$ & 3.01 & 2.83 & .24 & 2.69 & 2.57 & .12 \\
\hline $4 \ldots$ & 2.82 & 3.10 & .28 & 2.63 & 2.83 & .20 \\
\hline $5 \ldots$ & 3.06 & 2.97 & .09 & 2.92 & 2. 70 & .22 \\
\hline $6 \ldots$ & 2.94 & 2.56 & .38 & 2.51 & 2.42 & .09 \\
\hline 7.. & 2.84 & 3.03 & .19 & 2.66 & 2.86 & .20 \\
\hline 9. & 3.21 & 3.05 & .16 & 2.83 & 2.84 & .01 \\
\hline 10. & 2.98 & 2.87 & .11 & 2.59 & 2. 70 & .11 \\
\hline & 2.59 & 2.66 & .07 & 2.34 & 2.57 & .23 \\
\hline 12. & 2.81 & 2.62 & .19 & 2.59 & 2.52 & .07 \\
\hline 13. & 3.47 & 3.62 & .15 & 3.04 & 3.35 & .31 \\
\hline 14. & 2.61 & 2.54 & .07 & 2.44 & 2.42 & .02 \\
\hline & 2.54 & 2.46 & .08 & 2.25 & 2.29 & .04 \\
\hline 16. & 2.71 & 2.87 & .16 & 2.25 & 2.71 & .46 \\
\hline 17. & 2.85 & 3.01 & .16 & 2.73 & 2.75 & .02 \\
\hline 18. & 2.99 & 3.13 & .14 & 2.85 & 2.91 & .06 \\
\hline & 2.78 & 2.77 & .01 & 2.61 & 2.33 & .28 \\
\hline 20. & 2.78 & 2.80 & .02 & 2.60 & 2.57 & .03 \\
\hline 21 . & 2.79 & 2.71 & .08 & 2.51 & 2.48 & .03 \\
\hline Average. & & $\ldots$ & .14 & & - & .13 \\
\hline
\end{tabular}

The above table shows a maximum difference of 0.38 per cent in the content of total nitrogen of the two lots of spikes from one plant, and of 0.46 per cent in the content of proteid nitrogen. The average difference is only 0.14 per cent and 0.13 per cent, respectively.

These tables give unmistakable evidences that the average composition of a spike of wheat may be judged from the analysis of a row of its spikelets, and that the average composition of all of the spikes of a wheat plant is shown by an analysis of one-half the number. In practice it is better to take as the sample for analysis one row of spikelets from each spike, and the remaining row of spikelets from each spike for planting.

In order to ascertain what variation occurs between the several spikes on a single wheat plant, analyses were made of each spike from a number of plants. On some plants there were more spikes than on others, but every spike on each plant was analyzed. In the following tabulation of these analyses the percentage of proteid nitrogen is stated. 
TABLE 17.-Analyses of spikes of wheat, showing difference in proteid nitrogen.

\begin{tabular}{|c|c|c|c|c|c|c|}
\hline \multirow{2}{*}{ Spike. } & \multicolumn{6}{|c|}{ Percentage oi proteid nitrogen. } \\
\hline & Plant 23. & Plant 24. & Plant 25. & Plant 26. & Plant 27. & Plant 29. \\
\hline 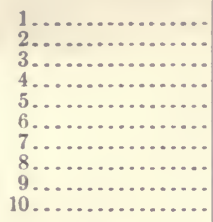 & $\begin{array}{l}2.33 \\
2.69 \\
2.37 \\
2.36 \\
2.15 \\
2.31 \\
2.09 \\
2.71 \\
2.32 \\
2.37\end{array}$ & \begin{tabular}{r}
2.46 \\
2.73 \\
2.35 \\
2.11 \\
2.19 \\
2.21 \\
2.53 \\
\hdashline$\ldots .$. \\
\hdashline \\
\end{tabular} & $\begin{array}{l}2.31 \\
2.36 \\
2.47 \\
2.59 \\
2.35 \\
2.39 \\
2.39 \\
2.60 \\
2.54 \\
2.83\end{array}$ & $\begin{array}{l}2.73 \\
3.02 \\
2.80 \\
2.60 \\
2.53 \\
2.37 \\
2.72 \\
2.37 \\
2.61 \\
2.45\end{array}$ & 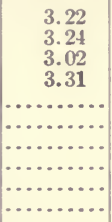 & $\begin{array}{l}2.38 \\
2.60 \\
3.03 \\
3.00 \\
2.34 \\
2.71 \\
2.21 \\
\cdots 2.60 \\
2.30\end{array}$ \\
\hline Maximum.... & 2.69 & 2.73 & 2.83 & 3.02 & 3.31 & 3.03 \\
\hline Average....... & 2.37 & 2.37 & 2.48 & 2.62 & 3.20 & $2.5 \pi$ \\
\hline Minimum.... & 2.09 & 2.11 & 2.31 & 2.37 & 3.02 & 2.21 \\
\hline $\begin{array}{l}\text { Greatest dif- } \\
\text { ference..... }\end{array}$ & .60 & .62 & .52 & .65 & .29 & .82 \\
\hline
\end{tabular}

These results show that there may be large differences between the proteid nitrogen content of spikes on the same plant. They do not, however, indicate that the determination of the average composition of the kernels on a plant is not a safe guide for selecting breeding stock. If the plant is the unit in reproduction, whether the plant reproduces itself from one seed or another does not affect its hereditary qualities in very marked degree.

It is evident, from a comparison of the variations that occur in the composition of the spikes from a single plant, and of the kernels on a single spike, that it is impossible to do more than obtain a reasonably close estimate of the composition of the kernels either on a part or on the whole of a plant. It therefore becomes desirable to obtain as closely as possible the average composition of the unit of reproduction. If the plant as a whole, and not any particular part, is this unit, the average composition of all of the kernels on the plant is a much safer guide as a basis for selection than is the average composition of the kernels of any part of it. One row of spikelets from each spike should therefore give the best sample for analysis.

In Table 18 is given a statement of the percentage of proteid nitrogen in the dry matter of the kernels on a row of spikelets of 800 spikes of wheat of the Turkish Red variety. These spikes were taken from a field of wheat, and were selected with reference to length of head, plumpness of kernel, uprightness of straw, freedom from rust, etc. They are therefore not spikes in which high nitrogen content is likely to be due to immaturity or arrested development. " Variations in the nitrogen content of different plants may in some degree be due to a larger or smaller supply of available nitrogen, although all were taken from the same field. Variations due to climate are, of course, precluded, as all grew during the same season. 
TABLE 18.-Variations in content of proteids.

\begin{tabular}{|c|c|c|c|c|c|c|c|c|}
\hline \multirow[b]{2}{*}{$\begin{array}{c}\text { Record } \\
\text { number. }\end{array}$} & \multicolumn{2}{|c|}{ Percentage of- } & \multirow[b]{2}{*}{$\begin{array}{l}\text { Record } \\
\text { number. }\end{array}$} & \multicolumn{2}{|c|}{ Percentage of- } & \multirow[b]{2}{*}{$\begin{array}{l}\text { Record } \\
\text { number. }\end{array}$} & Percent & tage of - \\
\hline & $\begin{array}{c}\text { Proteid } \\
\text { nitrogen } \\
\text { in water- } \\
\text { free } \\
\text { material. }\end{array}$ & $\begin{array}{l}\text { Proteids } \\
\text { (proteid } \\
N . \times 5.7)\end{array}$ & & $\begin{array}{c}\text { Proteid } \\
\text { nitrogen } \\
\text { in water- } \\
\text { free } \\
\text { material. }\end{array}$ & $\begin{array}{l}\text { Proteids } \\
\text { (proteid } \\
\mathrm{N} . \times 5.7)\end{array}$ & & $\begin{array}{c}\text { Proteid } \\
\text { nitrogen } \\
\text { in water- } \\
\text { free } \\
\text { material. }\end{array}$ & $\begin{array}{l}\text { Proteids } \\
\text { (proteid } \\
\text { N. } \times 5.7 \text { ). }\end{array}$ \\
\hline & 2.25 & 12.82 & 78. & 3.40 & 19.38 & 155. & 1.99 & 11.37 \\
\hline & 3.04 & 17.33 & 79 & 3. 33 & & & 3.03 & 17.20 \\
\hline & 2.45 & 13.96 & 80. & 3.79 & 21.60 & 157. & 2.07 & 11.87 \\
\hline 4.. & 3.14 & 17.90 & 81. & 3.63 & 20.69 & & 2.75 & 15. \\
\hline & 2.86 & 16.30 & 82. & 2.68 & 15.28 & 159. & 2.82 & 16.07 \\
\hline 6. & 2.83 & 16.13 & 83. & & & 100 & 3.06 & 17.44 \\
\hline & 3.67 & 20.92 & 84. & 2.46 & 14.02 & 161. & 2.54 & 14.48 \\
\hline 8. & 3.42 & 19.49 & 85. & 2.62 & 14.93 & 162. & 3.33 & 1008 \\
\hline & 2.36 & 13.45 & 86 & 2.87 & 16. & 163. & 2.73 & 15.56 \\
\hline & 2.2 & 13.00 & 87. & 2.89 & 16.86 & 164. & 2.47 & 14.08 \\
\hline 11. & 2.98 & 16.99 & 88. & 2.44 & 13.91 & 16 & 3.22 & 18. 35 \\
\hline 12. & 3.51 & 20.01 & 89. & 3.56 & 20.29 & 166. & 2.80 & 15.96 \\
\hline 13. & 3.63 & 20.69 & 90. & 3.76 & 21.43 & 167. & & \\
\hline 14. & 2.4 & 14.14 & 91. & & & 168. & 3.59 & 20.46 \\
\hline $15 .$. & 2.30 & 13.11 & 92. & 3.41 & 19.44 & 169. & 2.52 & 13.72 \\
\hline 16. & 3.48 & 19.84 & 93. & 2.30 & 13.11 & 170. & 2.72 & 15.50 \\
\hline 17. & 3.55 & 20.23 & 94. & & & 171. & 3.28 & 18.70 \\
\hline & 3. & 18.87 & 95 & $\cdots$ & & 172 . & 2.74 & 15. 62 \\
\hline 19. & 2.30 & 13.11 & 96. & 2.75 & 15.67 & 173. & 3.07 & 17.54 \\
\hline & 2.52 & 14.36 & & 4.07 & 23.20 & 174. & 3.75 & 21.43 \\
\hline 2 & 2.93 & 16.70 & 98. & 3.28 & 18.70 & 175. & 3.46 & 19.74 \\
\hline 22 & 3.25 & 18.52 & 99. & 3.24 & 17 & 176. & 3.09 & 17.67 \\
\hline 23. & & & 100. & 2.15 & 12.25 & 177. & 3.56 & 20.34 \\
\hline $24 .$. & 2.84 & 16.19 & 1 & 3.12 & 17.78 . & 178. & & \\
\hline & 2.73 & 15.56 & 2. & 3.00 & 17.10 & 179. & 3.85 & 21.95 \\
\hline 26 . & 3.55 & 20.23 & 103. & 2.87 & 16. 36 & 18 & 7 & \\
\hline & 2.33 & 13. 28 & & 3. & 20. & & 2. & \\
\hline 28. & 2.65 & 15.11 & & 2.61 & 14.88 & & 6 & \\
\hline & 2.82 & 16.07 & & 2.01 & 11 & 1 & 2. & 11. 73 \\
\hline 3 & 2.70 & 15.39 & & 2.68 & 15. & & 3.77 & 21.53 \\
\hline & 1.84 & 10.49 & & 3.10 & 7 & & 0 & 43 \\
\hline 3 & 3.10 & 17.67 & & 2.58 & 14.71 & 1 & 3.97 & 22.63 \\
\hline 3 & 2.86 & 16.30 & & 2.76 & 15 & & & \\
\hline & 2. & 12.31 & & 4.30 & 24 . & & 2. & \\
\hline & 2.58 & 14.71 & & 2.89 & 16. & & 3 & \\
\hline & 3. & 18.35 & & 2. & & & 3. & 8.52 \\
\hline 37. & 3.49 & 19.89 & & 2.68 & 15. & & 3.24 & 18. 52 \\
\hline & 2.76 & 15.73 & & 1.71 & 9 & 1 & 3.12 & 17.80 \\
\hline 3 & 2.96 & 16.87 & & 2.59 & 14. & 1 & 2.40 & 13. 72 \\
\hline & 2.86 & 16.30 & & 3.31 & 18.87 & & & 58 \\
\hline & 3.50 & 19.95 & 118 & & & & 3 & 18.99 \\
\hline & 3.05 & 17. & & 2.17 & & & & \\
\hline & 2.8 & 16.42 & & 2.88 & 16.42 & & & 16 \\
\hline & 2. & 15.67 & & & & & 8 & \\
\hline & 26 & 14.8 & & 1.33 & 7.58 & & 2. & 17 \\
\hline 4 & 2.50 & 14 & & 2.54 & 14. & & 3.23 & 18 \\
\hline & 3. & 17. & & 3.20 & & & & \\
\hline & 3.1 & 18. & & 2.04 & 11. & & .12 & 7.83 \\
\hline & 2.8 & 16. & & & & & & \\
\hline & 2.80 & 15. & & 2.8 & 16. & & & \\
\hline & 3. & & & 2.9 & & & & \\
\hline & 2.8 & 16. & & 2.8 & & & & 15 \\
\hline & 3. & 18. & & 2. & & & & \\
\hline & 2.9 & 16. & & 3.18 & 18.13 & & & \\
\hline & 3. & 218 & & ... & & & 1 & 76 \\
\hline & 3.38 & 19.27 & & & & & 2.94 & \\
\hline & 3.1 & 17.73 & & ... & $\cdots$ & & 2.89 & 16. 52 \\
\hline & 3. & 18. & & & & & & \\
\hline & 3. & 17. & & & & & & \\
\hline & 3.0 & 17.21 & & 2.13 & & & & \\
\hline & 1.7 & 10. & & 3. & 17.56 & & & \\
\hline & 2.6 & & & 1.37 & 7.81 & & & \\
\hline & 3.3 & 19. & & & & & & \\
\hline & 2.4 & 14. 19 & & 2.57 & 14.65 & & 2.58 & 14. 72 \\
\hline & 2.5 & 14. & & 2.75 & & & & \\
\hline & 2.12 & 12. & & 3. & & & 3.19 & 18 \\
\hline & 2. & 15. & & 3.17 & & & & 22 \\
\hline & 2.46 & 14. & & 2.09 & 11 & 2 & 2.93 & 16.71 \\
\hline & 2.3 & 13. & & 2.75 & & 223. & 3.30 & \\
\hline & 2.93 & 16.70 & & 2.42 & 13. & & 3. 65 & 20.8 \\
\hline & 2.32 & 13. 22 & & 2.68 & 15. & & 3.54 & 20.23 \\
\hline & 2.20 & 12.54 & & 2.25 & & & & 17. \\
\hline & 2.58 & 14.71 & & 2.61 & 14. & 227 . & 2.71 & 15.46 \\
\hline & 2.5 & 14.71 & & 1.51 & & & 3. 39 & 19. 36 \\
\hline & 3.22 & 13.35 & & 1.64 & 9. & 229 . & 2.96 & 16. 88 \\
\hline & & & & 2.93 & & & 2.54 & 14.46 \\
\hline & & & 154 & 2.85 & 16.24 & 231 & 3.11 & 17.73 \\
\hline
\end{tabular}


TABLE 1S.-Variations in content of proteids - Continued.

\begin{tabular}{|c|c|c|c|c|c|c|c|c|}
\hline \multirow[b]{2}{*}{$\begin{array}{c}\text { Record } \\
\text { number. }\end{array}$} & \multicolumn{2}{|c|}{ Percentage of - } & \multirow[b]{2}{*}{$\begin{array}{c}\text { Record } \\
\text { number. }\end{array}$} & \multicolumn{2}{|c|}{ Percentage of- } & \multirow[b]{2}{*}{$\begin{array}{c}\text { Record } \\
\text { number. }\end{array}$} & \multicolumn{2}{|c|}{ Percentage of- } \\
\hline & $\begin{array}{c}\text { Proteid } \\
\text { nitrogen } \\
\text { in water- } \\
\text { free } \\
\text { material. }\end{array}$ & $\begin{array}{l}\text { Proteids } \\
\text { (proteid } \\
\mathbf{N} \times 5.7)\end{array}$ & & $\begin{array}{c}\text { Proteid } \\
\text { nitrogen } \\
\text { in water- } \\
\text { free } \\
\text { material. }\end{array}$ & $\begin{array}{l}\text { Proteids } \\
\text { (proteid } \\
\text { N. } \times 5.7 \text { ). }\end{array}$ & & $\begin{array}{c}\text { Proteid } \\
\text { nitrogen } \\
\text { in water- } \\
\text { free } \\
\text { material. }\end{array}$ & $\begin{array}{l}\text { Proteids } \\
\text { (proteid } \\
\mathrm{N} \times 5.7 \text { ). }\end{array}$ \\
\hline 232. & 3.11 & 17.73 & 309. & 3. 74 & 21.36 & 386. & 2.52 & $15.0^{-}$ \\
\hline 33. & 3.31 & 18.92 & 310. & 3.15 & 18.01 & 387. & 2.73 & 15.59 \\
\hline 234. & 3.23 & 18.43 & 311. & 2.99 & 17.07 & 388. & 3.05 & 17.41 \\
\hline 35. & 3.65 & 20.82 & 312 . & 3.48 & 19.88 & 389. & 2.95 & 16.87 \\
\hline 236 . & 3.18 & 18.17 & 313. & 3.52 & 20.11 & 390. & 3.22 & 18. 36 \\
\hline 237. & $4.8 \bar{i}$ & 27.79 & 314. & 3.16 & 18.03 & 391. & 3.26 & 18.60 \\
\hline 8. & 2.69 & 15.38 & 315. & 2.75 & 15.68 & 392. & 2.93 & 16.74 \\
\hline , & 2.59 & 14. 77 & 316. & 3.35 & $\begin{array}{l}19.13 \\
19.54\end{array}$ & $\begin{array}{l}393 . . \\
394 .\end{array}$ & $\begin{array}{l}2.70 \\
2.77\end{array}$ & 15.41 \\
\hline 240 . & $\begin{array}{l}3.52 \\
2.76\end{array}$ & $\begin{array}{l}20.12 \\
15.75\end{array}$ & $\begin{array}{l}317 . . \\
315 . .\end{array}$ & $\begin{array}{l}3.42 \\
2.01\end{array}$ & $\begin{array}{l}19.54 \\
11.50\end{array}$ & $\begin{array}{l}394 . . \\
395 .\end{array}$ & $\begin{array}{l}2.77 \\
2.98\end{array}$ & $\begin{array}{l}15.81 \\
16.99\end{array}$ \\
\hline $\begin{array}{l}241 . . \\
242 . .\end{array}$ & 2.96 & 16. 89 & 319. & 2.86 & 16.33 & 396. & 2.28 & 13.02 \\
\hline 243. & 3.47 & 19. 78 & 320. & 2.98 & 17.00 & 397. & ....... & ....... \\
\hline 244 & 3.30 & 18.83 & $321 \ldots$ & $\begin{array}{l}3.42 \\
2.54\end{array}$ & $\begin{array}{l}19.54 \\
14.53\end{array}$ & 398. & 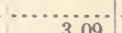 & $\cdots \cdots$ \\
\hline $\begin{array}{l}245 . \\
246 .\end{array}$ & $\begin{array}{l}3.64 \\
3.75\end{array}$ & & $\begin{array}{l}322 \ldots \\
323 \ldots\end{array}$ & $\begin{array}{l}2.54 \\
3.42\end{array}$ & & $\begin{array}{l}399 \ldots \\
400 . .\end{array}$ & $\begin{array}{l}3.09 \\
3.25\end{array}$ & $\begin{array}{l}17.65 \\
19.12\end{array}$ \\
\hline $\begin{array}{l}246 . \\
247 .\end{array}$ & 3.50 & 19.95 & 324 & 3.18 & 18.16 & 401. & 3.36 & 19.20 \\
\hline 248. & 3.64 & 20.78 & 325. & 3.45 & 19.70 & 402. & 2.32 & 13. 26 \\
\hline $\begin{array}{l}249 . \\
250\end{array}$ & $\begin{array}{l}3.21 \\
3.11\end{array}$ & $\begin{array}{l}18.32 \\
17.76\end{array}$ & 326. & 3,44 & 19.64 & 403. & $\begin{array}{l}3.03 \\
3.30\end{array}$ & 17.31 \\
\hline $250 \ldots$ & $\begin{array}{l}3.11 \\
3.46\end{array}$ & $\begin{array}{l}17.76 \\
19.73\end{array}$ & $\begin{array}{l}327 . \\
328\end{array}$ & $\begin{array}{l}3.4 t \\
3.60\end{array}$ & 20.55 & $\begin{array}{l}404 . \\
40.5 .\end{array}$ & $\begin{array}{l}3.30 \\
3.75\end{array}$ & $\begin{array}{l}18.83 \\
21.43\end{array}$ \\
\hline 32. & 2.54 & 14.52 & 329. & 2.87 & 16.39 & 406. & 2. 43 & 13.90 \\
\hline 253. & 3.63 & 20.71 & 330. & 2.61 & 14.93 & 407. & 3. 79 & 21. 63 \\
\hline 25. & 3.02 & 17.26 & $\begin{array}{l}331 \text {. } \\
332 .\end{array}$ & $2.57^{\circ}$ & 14.68 & $\begin{array}{l}408 . \\
409 .\end{array}$ & $\begin{array}{l}3.63 \\
3.59\end{array}$ & $\begin{array}{l}20.74 \\
20.47\end{array}$ \\
\hline 256. & 3.31 & 18.88 & 333 & 3.25 & 18.56 & 410. & 3.26 & $\begin{array}{l}20.47 \\
18.63\end{array}$ \\
\hline 58. & 3.37 & 19.24 & $\begin{array}{l}334 .- \\
335 .\end{array}$ & $\begin{array}{l}2.61 \\
2.81\end{array}$ & 15.70 & $\begin{array}{l}41 . \\
412 .\end{array}$ & $\begin{array}{l}3.15 \\
3.63\end{array}$ & $\begin{array}{l}17.95 \\
20.70\end{array}$ \\
\hline 259. & 3.84 & 21.89 & 336. & 3.35 & 19.11 & 413. & 3.76 & $\begin{array}{l}20.50 \\
21.51\end{array}$ \\
\hline 260. & 1.93 & 11.03 & 337. & 2.88 & 16.45 & 414. & 3.13 & 17.89 \\
\hline 261. & $\begin{array}{l}3.49 \\
\end{array}$ & 19.92 & $\begin{array}{l}338 . \\
339 .\end{array}$ & $\begin{array}{l}4.95 \\
3.33\end{array}$ & 28.23 & 415. & 2.44 & 13.93 \\
\hline $\begin{array}{l}262 . \\
263 .\end{array}$ & $\begin{array}{l}3.19 \\
3.24\end{array}$ & $\begin{array}{l}18.21 \\
18.48\end{array}$ & $\begin{array}{l}339 . \\
340 .\end{array}$ & $\begin{array}{l}3.33 \\
2.73\end{array}$ & $\begin{array}{l}19.01 \\
15.61\end{array}$ & $\begin{array}{l}416 . \\
417 .\end{array}$ & $\begin{array}{l}3.23 \\
3.79\end{array}$ & $\begin{array}{l}18.44 \\
21.65\end{array}$ \\
\hline 264 . & 3. 36 & 19.20 & 341. & 2.97 & 16.94 & 418. & $\begin{array}{l}3.19 \\
3.05\end{array}$ & $\begin{array}{l}21.60 \\
17.39\end{array}$ \\
\hline 26.5 & 3.29 & 18.80 & 342. & 2.60 & 14.82 & 419. & 2.85 & 16.28 \\
\hline $26 \%$ & 3.10 & 17.70 & 343. & 2.50 & 14.27 & 420. & 3. 73 & 21.27 \\
\hline $\begin{array}{l}26 \\
26\end{array}$ & $\begin{array}{l}3.18 \\
4\end{array}$ & $\begin{array}{l}18.18 \\
23.39\end{array}$ & $\begin{array}{l}344 . \\
345\end{array}$ & $\begin{array}{l}2.93 \\
2.55\end{array}$ & 16.71 & 421. & $\begin{array}{l}2.53 \\
3.53\end{array}$ & 14. 45 \\
\hline & $\begin{array}{l}4.10 \\
3.20\end{array}$ & $\begin{array}{l}23.39 \\
18.29\end{array}$ & $\begin{array}{l}345 . \\
346 .\end{array}$ & $\begin{array}{l}2.55 \\
2.55\end{array}$ & $\begin{array}{l}14.57 \\
14.55\end{array}$ & $\begin{array}{l}422 . . \\
423 .\end{array}$ & $\begin{array}{l}3.53 \\
3.14\end{array}$ & 20.12 \\
\hline $\begin{array}{l}269 . \\
270 .\end{array}$ & $\begin{array}{l}3.20 \\
3.36\end{array}$ & $\begin{array}{l}18.29 \\
19.19\end{array}$ & $\begin{array}{l}3+0 . \\
34 \%\end{array}$ & $\begin{array}{l}2.53 \\
2.44\end{array}$ & $\begin{array}{l}14.55 \\
13.92\end{array}$ & $\begin{array}{l}423 . \\
424 .\end{array}$ & $\begin{array}{l}3.14 \\
2.61\end{array}$ & $\begin{array}{l}17.90 \\
14.93\end{array}$ \\
\hline 271. & 3.39 & 19.34 & 348 . & 2.87 & 16.39 & 425. & 3. 29 & $\begin{array}{l}14.93 \\
18.81\end{array}$ \\
\hline 272 & 3.13 & 17.88 & 349. & 2.65 & 15 & 426 & 3. 08 & 17.60 \\
\hline 27 & 3. 39 & 19.78 & 350 . & 2.63 & 15.03 & 427. & 3. 06 & 17.46 \\
\hline 27 & 3.56 & 20.34 & 351. & 3.31 & 18 & 428. & 2. 59 & 14. 80 \\
\hline 275 & 3.32 & 18.96 & 352. & 3.04 & 17 & 42 & 3. 03 & 17.31 \\
\hline $2 \pi 6$. & 3.15 & 17.95 & 353. & 3.10 & 17 & 43 & 2. 81 & 16.06 \\
\hline 277. & 2.85 & 16.26 & 3.54 & 2.72 & 15 & $\begin{array}{l}43 . \\
43\end{array}$ & $\begin{array}{l}3.20 \\
3.00\end{array}$ & 18.25 \\
\hline 278. & 3.11 & 17.77 & 35. & 2.83 & 16 & $\begin{array}{l}43 \\
43\end{array}$ & $\begin{array}{l}3.00 \\
3.12\end{array}$ & 17.11 \\
\hline 279 & 3.78 & 21.60 & 356. & 2.91 & 16 & 43 & $\begin{array}{l}3.12 \\
2.85\end{array}$ & 17.80 \\
\hline 2 & $\begin{array}{l}3.70 \\
3.26\end{array}$ & 21.10 & 357. & $\begin{array}{l}2.36 \\
2.33\end{array}$ & & $\begin{array}{l}43 \\
43\end{array}$ & $\begin{array}{l}2.85 \\
3.53\end{array}$ & 16.28 \\
\hline $\begin{array}{l}281 \\
252\end{array}$ & $\begin{array}{l}3.26 \\
3.01\end{array}$ & 18.60 & $\begin{array}{l}358 \\
359\end{array}$ & 2.33 & 13 & $\begin{array}{l}435 \\
436\end{array}$ & $\begin{array}{l}3.53 \\
2.88\end{array}$ & 20.14 \\
\hline $\begin{array}{l}2 \\
2\end{array}$ & $\begin{array}{l}3.01 \\
3.85\end{array}$ & 19 & $\begin{array}{l}359 . \\
360\end{array}$ & $\begin{array}{l}2.97 \\
2.88\end{array}$ & 16 & $\begin{array}{l}436 \\
437\end{array}$ & $\begin{array}{l}2.88 \\
3.12\end{array}$ & 16. 44 \\
\hline $\begin{array}{l}2 \times 3 \\
281\end{array}$ & $\begin{array}{l}3.8 .5 \\
3.71\end{array}$ & 22.00 & $\begin{array}{l}360 . \\
361\end{array}$ & $\begin{array}{l}2.88 \\
2.94\end{array}$ & 16 & $\begin{array}{l}43 \\
43\end{array}$ & $\begin{array}{l}3.12 \\
2.66\end{array}$ & 17. 82 \\
\hline $\begin{array}{l}284 . \\
28.5\end{array}$ & $\begin{array}{l}3.71 \\
3.87\end{array}$ & 21.20 & $\begin{array}{l}361 \\
362\end{array}$ & $\begin{array}{l}2.94 \\
3.03\end{array}$ & 16 & $\begin{array}{l}43 \\
43\end{array}$ & & 15.20 \\
\hline 2 & $\begin{array}{l}3.87 \\
3.55\end{array}$ & 22.07 & $\begin{array}{l}362 . . \\
363\end{array}$ & $\begin{array}{l}3.0 \\
3.4\end{array}$ & 17 & $\begin{array}{l}439 \\
\mathbf{4 4 0}\end{array}$ & $\begin{array}{l}2.98 \\
2.35\end{array}$ & 16.99 \\
\hline 2 & $\begin{array}{l}3.55 \\
3.86\end{array}$ & 6 & $\begin{array}{l}36 \\
36\end{array}$ & $\begin{array}{l}3.49 \\
2.91\end{array}$ & 19. & $\begin{array}{l}44 \\
44\end{array}$ & $\begin{array}{l}2.35 \\
2.93\end{array}$ & 13. 44 \\
\hline 2 & $\begin{array}{l}3.86 \\
2.82\end{array}$ & $\begin{array}{l}22.04 \\
16.09\end{array}$ & 364 & $\begin{array}{l}2.91 \\
3.49\end{array}$ & 16 & $\begin{array}{l}441 \\
442\end{array}$ & $\begin{array}{l}2.93 \\
3.22\end{array}$ & 16. 72 \\
\hline & $\begin{array}{l}2.82 \\
2.52\end{array}$ & $\begin{array}{l}16.09 \\
14.40\end{array}$ & & $\begin{array}{l}3.4 \\
3.1\end{array}$ & & $\begin{array}{l}442 . \\
443 .\end{array}$ & $\begin{array}{l}3.22 \\
2.50\end{array}$ & 17.98 \\
\hline 289 & 4.00 & $\begin{array}{l}14.40 \\
22.81\end{array}$ & $\begin{array}{l}36 \\
36\end{array}$ & $\begin{array}{l}3.1 \\
3.3\end{array}$ & $\begin{array}{l}18 \\
19\end{array}$ & $\begin{array}{l}44 \\
44\end{array}$ & 2.37 & $\begin{array}{l}14.30 \\
13.56\end{array}$ \\
\hline 2 & 2.23 & 12.73 & $\begin{array}{l}36 \\
36\end{array}$ & $\begin{array}{l}3.3 \\
3.0\end{array}$ & & & 2.37 & $\begin{array}{l}13.56 \\
13.51\end{array}$ \\
\hline $\begin{array}{l}291 . \\
292 .\end{array}$ & 4.15 & 23.68 & & 3.33 & 19 & 44 & 3.75 & $\begin{array}{l}13.51 \\
21.37\end{array}$ \\
\hline 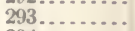 & 2.63 & 15.04 & 37 & 3.09 & 17. & 44 & 2.86 & 16. 33 \\
\hline & 2.56 & 14.60 & & $2.9 \varepsilon$ & 17 & & 3.13 & 16.67 \\
\hline & 3.05 & 17.41 & 37 & 3.30 & & 44 & 2. 76 & 15. 76 \\
\hline & 3.93 & 22.44 & & 2.86 & 16 & & 3. 61 & 20.62 \\
\hline 2 & 1.99 & 11.35 & 37 & 3.15 & 17. & & 2.92 & 16. 63 \\
\hline & 3.67 & 20.96 & & $\begin{array}{l}3.40 \\
2.59\end{array}$ & $\begin{array}{l}19.89 \\
14.76\end{array}$ & 4 & $\begin{array}{l}3.17 \\
3.15\end{array}$ & $\begin{array}{l}18.07 \\
17.86\end{array}$ \\
\hline 3 & 3.06 & 17.49 & 377. & 3.46 & & $\begin{array}{l}453 \\
454\end{array}$ & 3.14 & 17.92 \\
\hline & 3.08 & 17.61 & 378. & 2.74 & 15 & 45.5. & 2. 62 & 14.95 \\
\hline & 2.68 & 15.28 & 379. & 3.09 & 17.64 & 456. & 2. 71 & 15. 47 \\
\hline & 223 & 12.74 & 3 & $\begin{array}{l}2.35 \\
3.45\end{array}$ & $\begin{array}{l}13.42 \\
19.67\end{array}$ & $45 \%$. & $\begin{array}{l}3.14 \\
3.18\end{array}$ & $\begin{array}{l}17.92 \\
18.20\end{array}$ \\
\hline & $\begin{array}{l}2.23 \\
3.07\end{array}$ & $\begin{array}{l}12.74 \\
17.52\end{array}$ & $\begin{array}{l}381 . \\
382 .\end{array}$ & $\begin{array}{l}3.40 \\
3.22\end{array}$ & 18. & $\begin{array}{l}45 . \\
459 .\end{array}$ & 2.60 & 14.84 \\
\hline & 2.50 & 14.30 & 383. & 2.96 & 16.88 & 460 . & 3.91 & 22.29 \\
\hline & 3. 19 & 18.20 & 384. & 3. 55 & 20. 26 & $\begin{array}{l}461 . \\
469\end{array}$ & & \\
\hline & 2.84 & 16.22 & 38.5. & 3. 79 & 21.62 & 462. & 2. 39 & 13.64 \\
\hline
\end{tabular}


TABLE 18.-Variations in content of proteids-Continued.

\begin{tabular}{|c|c|c|c|c|c|c|c|c|}
\hline \multirow[b]{2}{*}{$\begin{array}{c}\text { Record } \\
\text { number. }\end{array}$} & \multicolumn{2}{|c|}{ Percentage of- } & \multirow[b]{2}{*}{$\begin{array}{l}\text { Record } \\
\text { number. }\end{array}$} & \multicolumn{2}{|c|}{ Percentage of- } & \multirow[b]{2}{*}{$\begin{array}{l}\text { Record } \\
\text { number. }\end{array}$} & \multicolumn{2}{|c|}{ Percentage of- } \\
\hline & $\begin{array}{c}\text { Proteid } \\
\text { nitrogen } \\
\text { in water- } \\
\text { free } \\
\text { material. }\end{array}$ & $\begin{array}{l}\text { Proteids } \\
\text { (proteid } \\
\text { N. } \times 5.7) .\end{array}$ & & $\begin{array}{c}\text { Proteid } \\
\text { nitrogen } \\
\text { in water- } \\
\text { free } \\
\text { material. }\end{array}$ & $\begin{array}{l}\text { Proteids } \\
\text { (proteid } \\
\text { N. } \times 5.7)\end{array}$ & & $\begin{array}{c}\text { Proteid } \\
\text { nitrogen } \\
\text { in water- } \\
\text { free } \\
\text { material. }\end{array}$ & $\begin{array}{l}\text { Proteids } \\
\text { (proteid } \\
\text { N. } \times 5.7) \text {. }\end{array}$ \\
\hline 463. & 2.49 & 14.24 & 540. & 3.17 & 18. 12 & 617. & 3.12 & 17.83 \\
\hline 64. & .98 & 1.29 & & 3.09 & 17.66 & & 2.67 & 15.27 \\
\hline 465. & 3.32 & 18.97 & 542. & 3.33 & 19.01 & 619. & 3.59 & 20.49 \\
\hline 466. & 2.98 & 17.01 & 543. & 3.50 & 19.96 & 620. & 2.68 & 15. 30 \\
\hline 467. & 2.89 & 16. 48 & 544. & 1.29 & 7.37 & 621. & 2.24 & 12.79 \\
\hline 468. & 2.95 & 16.82 & 545. & 2.10 & 11.98 & 622. & 3.19 & 18.23 \\
\hline & 2.74 & 15. 62 & 546 . & 2.54 & 14. 49 & 623. & 3.52 & 20.09 \\
\hline 470. & 2.80 & 15.97 & 547. & 2.73 & 15. 59 & 624. & 2.67 & 15. 27 \\
\hline 471. & 2.24 & 12.79 & 548 & 3.01 & 17. 21 & 625. & 2.68 & 15 \\
\hline 472. & 2.49 & 14. 22 & 549. & 2.50 & 14. 30 & 626. & 2.69 & 15. 38 \\
\hline 473. & 2.76 & 15.78 & 550. & 2.84 & 16. 20 & 627. & 2.88 & 16.44 \\
\hline 474. & 2.80 & 15. 97 & 551 . & 2.9 & 17. 08 & 628. & 3.68 & 21.01 \\
\hline 475. & 2.95 & 16. 83 & 552. & 2.30 & 13.11 & 629 & 3.47 & 19.82 \\
\hline 476. & 2.52 & 14. 39 & 553. & 3.21 & 18. 35 & 630. & & 14. 16 \\
\hline 477. & 2.95 & 16.85 & 554. & 2.91 & 16. 59 & 631. & 3.39 & 19.35 \\
\hline 478. & 3.15 & 18.00 & 555. & 3.16 & 18.06 & 632. & 3.22 & 18. 41 \\
\hline 479. & 2.27 & 12. 96 & 556. & 3.0 & 17.26 & 633. & 1.64 & 9.38 \\
\hline 80. & 2.72 & 15. 53 & 5 & 3. & 18. 86 & 634. & & 11. \\
\hline 481. & 3.04 & 17.38 & 558. & 3.2 & 18. 58 & 635. & 2 & 19.52 \\
\hline 482 & 3.15 & 17.97 & 559. & 2.9 & & 636. & & \\
\hline 483. & 2.60 & 14.86 & 560 . & 3. & 18. & 637. & & 15. \\
\hline 484. & 3.45 & 19.71 & 561. & 3. & 17.13 & 638. & & 25 \\
\hline 485. & 2.59 & 14.81 & 562. & 1. & 6.40 & 639. & & 18. \\
\hline 486. & 2.68 & 15.31 & 563. & 2.3 & 13. 49 & 640. & & 16. \\
\hline 487. & 3.01 & 17.18 & 564. & 3.83 & 21.84 & 641 . & & 19. \\
\hline 488. & 2.41 & 13.77 & 565 . & 340 & & & 7 & 14. \\
\hline 489. & 3.45 & 19.70 & 566 . & 3.49 & 19.49 & 643. & & \\
\hline 490. & 2.46 & 14.02 & 567. & 3.0 & 17.57 & 644. & 3. & 19 \\
\hline & 2.87 & 16.40 & & 2.1 & 12 & 64 & & \\
\hline 492. & 2.06 & 11.78 & 569 . & 3.0 & 17.29 & 646. & & 21 \\
\hline 493 & 3.18 & 18.16 & 570 . & 3.2 & 18.27 & 64 & 2 & 16 \\
\hline 494. & 2.45 & 13.97 & 57 & 2.5 & 14. 37 & 64 & 2. & 14. \\
\hline 495. & 2.36 & 13.45 & 5 & 3.1 & 17.82 & 64 & 6 & 14.63 \\
\hline $\begin{array}{l}496 . \\
497\end{array}$ & 2.52 & 14. 38 & 573 & 2.52 & 14.41 & 650. & 2.59 & 14.82 \\
\hline 497 & $\begin{array}{l}2.84 \\
2.82\end{array}$ & $\begin{array}{l}16.21 \\
16.08\end{array}$ & $\begin{array}{l}574 . \\
575 .\end{array}$ & $\begin{array}{l}3.25 \\
3.17\end{array}$ & 18.53 & 651 . & 283 & \\
\hline 499. & $\begin{array}{l}2.82 \\
2.97\end{array}$ & $\begin{array}{l}16.08 \\
16.95\end{array}$ & $\begin{array}{l}575 \\
576 .\end{array}$ & $\begin{array}{l}3.17 \\
2.52\end{array}$ & 18. 10 & $\begin{array}{l}652 . \\
653 .\end{array}$ & $\begin{array}{l}2.83 \\
2.50\end{array}$ & $\begin{array}{l}16.19 \\
14.31\end{array}$ \\
\hline 500 . & 3.06 & $\begin{array}{l}10.90 \\
17.48\end{array}$ & 577. & $\begin{array}{l}2.02 \\
3.09\end{array}$ & $\begin{array}{l}14.40 \\
17.61\end{array}$ & $\begin{array}{l}653 . \\
654 .\end{array}$ & & $\begin{array}{l}14.31 \\
14.81\end{array}$ \\
\hline 501. & 2.64 & 15.09 & 578 & 2.7 & 15.60 & 6 & & 18. 30 \\
\hline 502. & 2.72 & 15.56 & 579. & 3.35 & 19.10 & 656 & 2. & 14. 61 \\
\hline $\begin{array}{l}503 . \\
504\end{array}$ & 2. 31 & $\begin{array}{l}13.19 \\
17.48\end{array}$ & $\begin{array}{l}58 \\
58\end{array}$ & 370 & $91 \mathrm{~A}$ & 657. & 2.55 & 14. \\
\hline 504. & $\begin{array}{l}3.06 \\
2.71\end{array}$ & $\begin{array}{l}17.48 \\
15.46\end{array}$ & $\begin{array}{l}58 \\
58\end{array}$ & $\begin{array}{l}3.79 \\
2.59\end{array}$ & $\begin{array}{l}21.61 \\
14.77\end{array}$ & 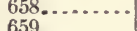 & $\ddot{2} \ddot{q}$ & \\
\hline & $\begin{array}{l}2.71 \\
2.49\end{array}$ & $\begin{array}{l}15 . \\
14 .\end{array}$ & 583. & 3.1 & 17.86 & 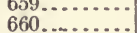 & 3. & 16. 70 \\
\hline & 3.13 & 17.85 & 584. & 3.49 & 19. & & & 56 \\
\hline 508 & 2.89 & 16.51 & & 3.0 & 17. & 62. & & 14 \\
\hline & 3.20 & 18. 29 & 5 & 3.27 & 18. & 63. & & 16. \\
\hline 510 & 2.93 & 16.71 & $\cdots$ & 2.5 & 14. & 6 & & \\
\hline 51. & 3.61 & 20.59 & & 2.8 & & 0 & & 19 \\
\hline 512 & 2.71 & 15.45 & 58 & 2.8 & 16. & 6 & & 13 \\
\hline & 2.86 & 16. 33 & & 2.8 & 16. & & & 13. \\
\hline & 2. 41 & 13. & & 3. & 17. & & 2. & 14 \\
\hline & 2.27 & 12. & . & 3.2 & 18. & 6 & & \\
\hline & 3.28 & 18. & & 2. & & $a$ & & \\
\hline 617 & 2.36 & 13. & 5 & 3. & 18 & 6 & & 13 \\
\hline & 3.64 & 20.75 & & 3. & & & & 28.15 \\
\hline & 2.81 & 16.03 & & 3.0 & 17. & 673. & 3. & 19. 35 \\
\hline & 2.54 & 14. & & 3. & & & & \\
\hline & 2.68 & 15. & & 2. & 13 & & & 19 \\
\hline & 3. 12 & 17. & 5 & 3.1 & & & & \\
\hline & 2.99 & 17. & & 2. & 16. & 677. & & 14 \\
\hline 59 & 1.93 & 11. & 60 & 2. & 11. & 678. & 3.09 & \\
\hline & 2.51 & 14. & 60 & 3.64 & 20. & 67 & & 14 \\
\hline & 1.71 & 9. & 603. & 2.56 & 14. & 680. & 2.30 & 13.13 \\
\hline & 3.15 & 17. & & 2. & & & & 19.17 \\
\hline & 2.35 & 13. & & 2.5 & 14. & 682. & 2. 49 & 14. 20 \\
\hline 52 & 2.88 & 16. & & 3. & & 683. & & 15.41 \\
\hline & 2.64 & 15. & 60 & 3.0 & 17. & 68 & 3.59 & 20. \\
\hline & 2.97 & 16. & 6 & 3. & & & & 23.06 \\
\hline & 2.75 & 15. & 60 & 2. & 15. & 68 & 2. & 15. \\
\hline & 3.22 & 18. & & 3.51 & 20. & 687. & 2.83 & 16. 13 \\
\hline 534 & 2.95 & 16.82 & 611. & 3.00 & 17. & 688. & 2.65 & 15.12 \\
\hline 535 & 3.03 & 17.2 & 612. & 3.26 & 18. 62 & 689. & 2.68 & 15. 28 \\
\hline & 2.57 & 14. 6 & 613. & 3.84 & 21. & 69 & & 19.26 \\
\hline 537 & 2.88 & 16.47 & 614. & 2.77 & 15. & 691. & 3.04 & 17.33 \\
\hline & 2.64 & 15.0 & & 2.7 & 15. & 692. & & 16.04 \\
\hline & 3.76 & 21.46 & 61 & 3.72 & 21.2 & 693. & 2.35 & 13.7 \\
\hline
\end{tabular}


TABLE 18.-Variations - in content of proteids - Continued.

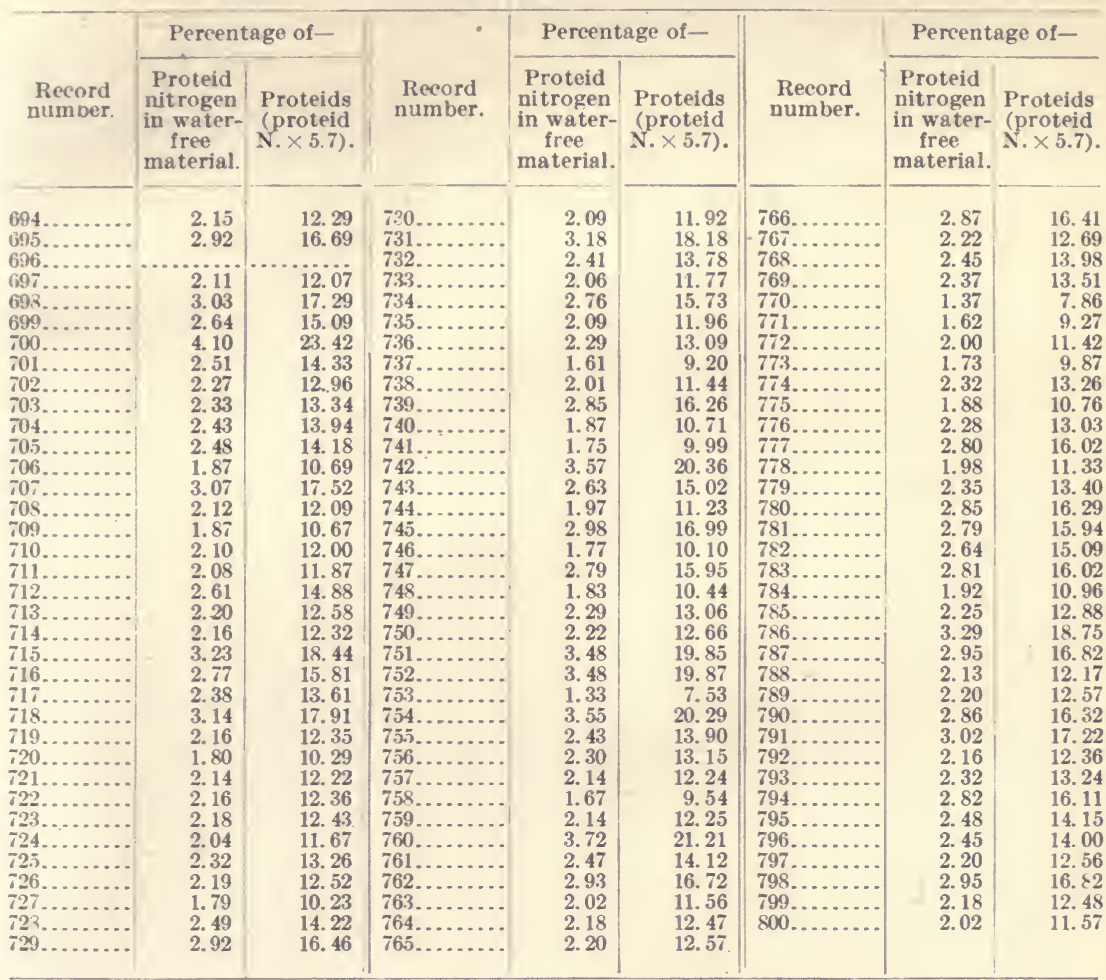

It will be noticed that there is a very large range of variation in the proteid nitrogen content of these wheats, running from 1.12 to 4.95 per cent. By referring to Table 8 , it will be seen that an equally large variation occurred between the plants when the whole plant was sampled. In the 351 analyses the nitrogen ranges from 1.20 to 5.85 per cent. This is due in the main to the ability of the plant to gather nitrogen from the soil. In no one of the experiments to ascertain the effect of nitrogenous manures on the composition of wheat has there been an increase of more than a few tenths of 1 per cent, even when the nitrogenous fertilizer was added to an exhausted soil. It is, therefore, not likely that such large variation in nitrogen content could be due to irregularities in the supply of soil nitrogen. If this ability of the plant to store up a large amount of nitrogen in the kernel is hereditary, as results given later indicate, there is ample onportunity to develop by selection a strain of wheat of high nitrogen content. 


\section{A BASIS FOR SELECTION TO INCREASE THE QUANTITY OF PROTEIDS IN THE ENDOSPERM OF THE KERNEL.}

White bread flour, which constitutes the major portion of the wheat flour consumed in this country, is derived entirely from the endosperm of the wheat kernel. The portions of the kernel not entering into the flour are the germ and the seed coat, attached to each of which discarded constituents are portions of the endosperm. The larger part of the aleurone layer either adheres to the hull and constitutes the "bran" of commerce, or appears in the product known as "shorts," and sometimes in low-grade flour.

As it is the flour in which it is desired to increase the nitrogen, and as the flour consists entirely of the endosperm, it becomes desirable to have some way to determine the nitrogen content of the endosperm alone and to select for reproduction plants possessing a large amount of nitrogen in this portion of the kernel.

It is a question how this can best be done. A determination of gluten by the ordinary method of washing, to carry off the starch and fiber while the gluten is being worked in the hand, is not well adapted for use with the small quantities of wheat obtainable from a single plant. This also has the disadvantage that it gives no indication as to the quality of the gluten.

Determinations of gliadin and glutenin promise to be of some help in affording a basis for selection from individual plants. It has been shown by Osborne and Voorhees ${ }^{a}$ that the gluten of wheat is composed of gliadin and glutenin. It does not necessarily follow, however, that the sum of these two substances is a measure of the gluten content of the sample analyzed. Osborne and Campbell ${ }^{b}$ have stated that the embryo of the wheat kernel does not contain either gliadin or glutenin. This being the case, the sum of the gliadin and glutenin would represent these proteids in the endosperm, with, perhaps, a small amount in the hull.

A recent investigation by Nasmith $^{c}$ leads him to conclude that gliadin exists in all portions of the endosperm, including the aleurone layer, but that glutenin is contained only in the starch-bearing portion of the endosperm. A determination of glutenin may, therefore, give an indication of the gluten content of the wheat.

Table 19 shows the percentage of proteid nitrogen, the sum of the gliadin and glutenin nitrogen, the amounts in grams of proteid and of gliadin-plus-glutenin nitrogen in the average kernel, and the grams of proteid and of gliadin-plus-glutenin nitrogen in all of the kernels on each plant. The plants are grouped into those having

a American Chem. Jour., 1893, pp. 392-471.

${ }^{\iota}$ Connecticut Experiment Station Report, 1899, p. 305.

c Trans. Canad. Inst., 7 (1903), Univ. Toronto Studies, Physiol. Ser. (1903), No. 4. 
from 1 to 2 per cent proteid nitrogen, those having 2 to 2.5 per cent proteid nitrogen, etc. Table 20 gives the averages for each of the groups in Table 19.

TABLE 19.-Relation of gliadin-plus-glutenin nitrogen to proteid nitrogen.

1 TO 2 PER CENT PROTEID NITROGEN.

\begin{tabular}{|c|c|c|c|c|c|c|c|c|c|}
\hline \multirow[b]{2}{*}{ Record sumber. } & \multicolumn{2}{|c|}{$\begin{array}{c}\text { Pereentage } \\
\text { of - }\end{array}$} & \multirow[b]{2}{*}{$\begin{array}{l}\text { Num- } \\
\text { ber of } \\
\text { ker- } \\
\text { nels. }\end{array}$} & \multicolumn{6}{|c|}{ Weight (in grams) of- } \\
\hline & $\begin{array}{l}\text { Pro- } \\
\text { teid } \\
\text { nitro- } \\
\text { gen. }\end{array}$ & $\begin{array}{l}\text { Glia- } \\
\text { din- } \\
\text { plus- } \\
\text { glu- } \\
\text { tenin } \\
\text { nitro- } \\
\text { gen. }\end{array}$ & & $\begin{array}{l}\text { Ker- } \\
\text { nels. }\end{array}$ & $\begin{array}{l}\text { Average } \\
\text { kernel. }\end{array}$ & $\begin{array}{l}\text { Proteid } \\
\text { nitro- } \\
\text { gen in } \\
\text { kernels. }\end{array}$ & $\begin{array}{l}\text { Gliadin- } \\
\text { plus- } \\
\text { glutenin } \\
\text { nitro- } \\
\text { gen in } \\
\text { kernels. }\end{array}$ & $\begin{array}{c}\text { Proteid } \\
\text { nitrogen } \\
\text { in aver- } \\
\text { age ker- } \\
\text { nel. }\end{array}$ & $\begin{array}{l}\text { Gliadin- } \\
\text { plus-gln- } \\
\text { tenin ni- } \\
\text { trogen in } \\
\text { average } \\
\text { kernel. }\end{array}$ \\
\hline $\begin{array}{l}55307 \ldots \ldots \ldots \\
80305 \ldots \ldots \ldots \\
81705 \ldots \ldots \ldots\end{array}$ & $\begin{array}{l}1.89 \\
1.81 \\
1.98\end{array}$ & $\begin{array}{l}1.56 \\
1.77 \\
1.96\end{array}$ & $\begin{array}{l}342 \\
729 \\
465\end{array}$ & $\begin{array}{r}5.6864 \\
15.7835 \\
9.7922\end{array}$ & $\begin{array}{r}0.01663 \\
.02165 \\
.02106\end{array}$ & $\begin{array}{r}0.10747 \\
.25569 \\
.19388\end{array}$ & $\begin{array}{r}0.08871 \\
.27937 \\
.19193\end{array}$ & $\begin{array}{r}0.0003142 \\
.0003919 \\
.0004170\end{array}$ & $\begin{array}{r}0.0002594 \\
.0003832 \\
.0004128\end{array}$ \\
\hline Average... & 1.89 & 1. 76 & 512 & 10.4207 & .01978 & .19568 & .18667 & .0003744 & .0003518 \\
\hline
\end{tabular}

2 TO 2.5 PER CENT PROTEID NITROGEN.

\begin{tabular}{|c|c|c|c|c|c|c|c|c|c|}
\hline - & & & & & & & & & \\
\hline $21212 \ldots \ldots \ldots$ & 2.16 & 0.19 & 84 & 1. 7216 & 0.02049 & 0.03718 & 0.00327 & 0.0004427 & 0.0000389 \\
\hline $27205 \ldots \ldots \ldots$ & 2.41 & 1.70 & 891 & 16. 4061 & .01841 & .39539 & .27890 & .0004437 & .0003130 \\
\hline $27206 \ldots \ldots \ldots$ & 2.36 & 1.46 & 777 & 19. 1854 & .02469 & .45276 & .28010 & .0005827 & .0003605 \\
\hline $27505 \ldots \ldots \ldots$ & 2.12 & 1.65 & 539 & 12.0399 & .02183 & .24942 & .19866 & .0004627 & .0003602 \\
\hline $33107 \ldots \ldots \ldots$ & 2.35 & 2. 12 & 318 & 6. 1026 & .01919 & .14341 & .12643 & .0004510 & .0004163 \\
\hline $33605 \ldots \ldots \ldots \ldots$ & 2.39 & 1.92 & 301 & 7.0596 & .02345 & .16872 & .13554 & .0005605 & .0004502 \\
\hline $39205 \ldots \ldots \ldots$ & 2.11 & 1.84 & 1,031 & 21.5399 & .02089 & .45435 & .39635 & .0004407 & .0003844 \\
\hline $48106 \ldots \ldots \ldots$ & 2.38 & 1.80 & 608 & 11. 6655 & .01919 & .27765 & .20997 & .0004567 & .0003454 \\
\hline $48409 \ldots$ & 2.02 & 1.50 & 314 & 6.4302 & .02048 & .12989 & .09645 & .0004137 & .0003072 \\
\hline 55309 & 2.48 & 1.97 & 167 & 2.5160 & .01507 & .06240 & .04957 & .0003736 & .0002969 \\
\hline $55908 \ldots$ & 2. 42 & 1.96 & 562 & 12.2210 & .02175 & .29575 & .23953 & .0005262 & .0004263 \\
\hline $55909 \ldots$ & 2.30 & 1.66 & 302 & 9.2120 & .03050 & .21187 & .15292 & .0007016 & .0005063 \\
\hline $56206 \ldots$. & 2. 42 & 1.95 & 509 & 9.3093 & .01829 & .22529 & .18153 & .0004426 & .0003566 \\
\hline $56207 \ldots \ldots \ldots$ & 2.34 & 1.83 & 462 & 10.9073 & .02361 & .25522 & .19960 & .0005524 & .0004321 \\
\hline $57508 \ldots \ldots \ldots$ & 2.21 & 2.05 & 380 & 12.0728 & .03177 & .26680 & .24750 & .0007021 & .0006513 \\
\hline $65306 \ldots \ldots \ldots$ & 2.41 & 1.68 & 544 & 9.8298 & .01807 & .23690 & .16514 & .0004355 & .0003036 \\
\hline $65307 \ldots \ldots \ldots$ & 2.28 & 1.81 & 373 & 7. 0051 & .01878 & .15971 & .12680 & .0004282 & .0003399 \\
\hline $65308 \ldots \ldots . .$. & 2.09 & 1.95 & 583 & 11. 7066 & .02008 & .24468 & .22828 & .0004197 & .0003916 \\
\hline $74606 \ldots \ldots \ldots$ & 2.30 & 2.05 & 464 & 9.6451 & .02079 & .22184 & .19772 & .0004781 & .0004262 \\
\hline $81707 \ldots . .$. & 2.34 & .64 & 786 & 18. 3614 & .02336 & .42965 & 11750 & .0005466 & .0001495 \\
\hline $81708 \ldots . . .$. & 2.41 & 1.64 & 287 & 7.3993 & .02578 & .17833 & .12135 & .0006213 & .0004228 \\
\hline Average.. & 2.30 & 1.68 & 489.6 & 10.5874 & .02173 & .24272 & .17872 & .0004991 & .0003652 \\
\hline
\end{tabular}

2.5 TO 3 PER CENT PROTEID NITROGEN.

\begin{tabular}{|c|c|c|c|c|c|c|c|c|c|}
\hline 20706 . & 2.78 & 2.05 & 163 & 3.3138 & 0.02033 & 0.09212 & 0.06793 & 0.0005652 & 0.0004168 \\
\hline $20707 \ldots$ & 2.77 & 1.85 & 444 & 9.9070 & .02282 & .27443 & .18328 & .0006181 & .0004222 \\
\hline $20710 \ldots$ & 2.83 & 2.00 & 867 & 17.1115 & .01974 & .48428 & .34222 & .0005586 & .0003948 \\
\hline $21207 \ldots$ & 2.96 & .17 & 118 & 2. 3066 & .01955 & .06804 & .00392 & .0005766 & .0000332 \\
\hline $21305 \ldots .$. & 2.67 & 1.97 & 313 & 6.2514 & .02004 & .16691 & .12315 & .0005353 & .0003948 \\
\hline $21306 \ldots \ldots$ & 2.90 & .97 & 226 & 4. 1516 & .01837 & .12039 & $.0402 \pi$ & .0005327 & .0001782 \\
\hline $21805 \ldots$. & 2. 69 & .23 & 1,232 & 20.9290 & .01639 & .56299 & .04704 & .0004569 & .0000391 \\
\hline $21807 \ldots$ & 2. 73 & 2.11 & 377 & 9.4172 & .02498 & .25709 & .19870 & .0006664 & .0005271 \\
\hline $21808 \ldots$ & 2.57 & 1.96 & 1,156 & 19.7446 & .01708 & .50744 & .38700 & .0004389 & .0003348 \\
\hline $21809 \ldots$ & 2.73 & 2.18 & 418 & 8.0214 & .01919 & .21898 & .17487 & .0005238 & .0004183 \\
\hline $21905 \ldots$ & 2.64 & 2.18 & 791 & 14.3111 & .01809 & .37781 & .31198 & .0004777 & .0003944 \\
\hline $22205 \ldots$ & 2.81 & 1.97 & 283 & 2. 6965 & .00953 & .07577 & .05312 & .0002677 & .0001877 \\
\hline $22207 \ldots$ & 2.77 & 1.82 & 169 & 3.2787 & .01940 & .09082 & .05967 & .0005374 & .0003531 \\
\hline $26905 .$. & 2.76 & 2.09 & 326 & 6.4102 & .01966 & .17692 & .13398 & .0005427 & .0004109 \\
\hline $26906 \ldots$ & 2.71 & 1.82 & 228 & 4. 2376 & .01859 & .11481 & .07712 & .0005037 & .0003383 \\
\hline $26908 . .$. & 2.96 & 2.16 & 192 & 3.9797 & .02073 & .11780 & .08596 & .0006135 & .0004478 \\
\hline $26909 \ldots$ & 2.80 & 1. 88 & 180 & 2. 9999 & .01667 & .08400 & .05640 & .0004667 & .0003134 \\
\hline 27005. & 2.63 & 1.90 & 866 & 16.4120 & .01895 & .43164 & .31182 & .0004984 & .0003600 \\
\hline 27207 .. & 2.92 & 1.95 & 166 & 3. 3266 & .02004 & .09712 & .06487 & .0005850 & .0003908 \\
\hline $27305 .$. & 2.58 & 1.73 & 267 & 5.5666 & .02085 & .14362 & .09630 & .0005379 & .0003607 \\
\hline $27307 \ldots$ & 2.53 & .82 & 167 & 3.0850 & .01847 & .07805 & .02530 & .0004674 & .0001515 \\
\hline $27506 \ldots$ & 2.70 & 1.98 & 444 & 10.0005 & .02252 & .27003 & .19800 & .0006082 & .0004459 \\
\hline $27500 . .$. & 2.64 & 2.32 & 251 & $5.5 \geq 24$ & .02287 & .14608 & .12835 & .0006037 & .0005366 \\
\hline $27509 \ldots \ldots$ & 2.90 & 1.09 & 243 & 5. 3615 & .02206 & .15549 & .05844 & .0006399 & .0002405 \\
\hline
\end{tabular}


TABLE 19.-Relation of gliadin-plus-glutenin nitrogen to proteid nitrogen-Continued.

2.5 TO 3 PER CENT PROTEID NITROGEN-Continued.

\begin{tabular}{|c|c|c|c|c|c|c|c|c|c|}
\hline \multirow[b]{2}{*}{ Record number. } & \multicolumn{2}{|c|}{$\begin{array}{c}\text { Percentage } \\
\text { of - }\end{array}$} & \multirow[b]{2}{*}{$\begin{array}{l}\text { Num- } \\
\text { ber of } \\
\text { ker- } \\
\text { nels. }\end{array}$} & \multicolumn{6}{|c|}{ Weight (in grams) of- } \\
\hline & $\begin{array}{c}\text { Pro- } \\
\text { teid } \\
\text { nitro- } \\
\text { gen. }\end{array}$ & $\begin{array}{c}\text { Glia- } \\
\text { din- } \\
\text { plus- } \\
\text { glu- } \\
\text { tenin } \\
\text { nitro- } \\
\text { gen. }\end{array}$ & & $\begin{array}{l}\text { Ker- } \\
\text { nels. }\end{array}$ & $\begin{array}{c}\text { Average } \\
\text { kernel. }\end{array}$ & $\begin{array}{l}\text { Proteid } \\
\text { nitro- } \\
\text { gen in } \\
\text { kernels. }\end{array}$ & $\begin{array}{l}\text { Gliadin- } \\
\text { plus- } \\
\text { glutenin } \\
\text { nitro- } \\
\text { gen in } \\
\text { kernels. }\end{array}$ & $\begin{array}{c}\text { Proteid } \\
\text { nitrogen } \\
\text { in aver- } \\
\text { age ker- } \\
\text { nel. }\end{array}$ & $\begin{array}{l}\text { Gliadin- } \\
\text { plus-glu- } \\
\text { tenin ni- } \\
\text { trogen in } \\
\text { average } \\
\text { kernel. }\end{array}$ \\
\hline 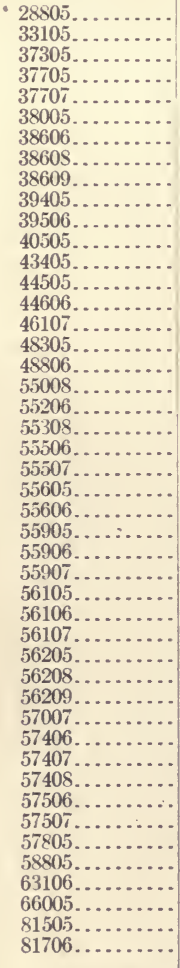 & $\begin{array}{l}2.91 \\
2.91 \\
2.96 \\
2.64 \\
2.93 \\
2.84 \\
2.63 \\
2.82 \\
2.74 \\
2.88 \\
2.93 \\
2.82 \\
2.92 \\
2.94 \\
2.90 \\
2.54 \\
2.87 \\
2.70 \\
2.60 \\
2.56 \\
2.54 \\
2.80 \\
2.63 \\
2.64 \\
2.58 \\
2.67 \\
2.81 \\
2.59 \\
2.73 \\
2.57 \\
2.96 \\
2.51 \\
2.61 \\
2.59 \\
2.65 \\
2.75 \\
2.62 \\
2.61 \\
2.80 \\
2.85 \\
2.87 \\
2.74 \\
2.79 \\
2.63 \\
2.94 \\
2.71 \\
\end{array}$ & $\begin{array}{l}1.55 \\
3.50 \\
2.29 \\
1.26 \\
2.10 \\
1.23 \\
1.39 \\
1.73 \\
1.34 \\
1.44 \\
2.06 \\
219 \\
1.18 \\
.70 \\
1.29 \\
2.08 \\
1.77 \\
.75 \\
1.58 \\
1.87 \\
.65 \\
2.20 \\
2.07 \\
1.96 \\
1.49 \\
1.75 \\
1.47 \\
1.61 \\
2.12 \\
2.09 \\
2.23 \\
1.85 \\
1.95 \\
2.21 \\
2.09 \\
2.13 \\
1.86 \\
1.64 \\
2.34 \\
1.55 \\
2.68 \\
2.11 \\
2.20 \\
2.18 \\
2.65 \\
2.03 \\
\end{array}$ & $\begin{array}{r}87 \\
132 \\
309 \\
461 \\
193 \\
139 \\
401 \\
158 \\
293 \\
447 \\
67 \\
170 \\
124 \\
340 \\
124 \\
478 \\
473 \\
547 \\
944 \\
578 \\
397 \\
866 \\
504 \\
500 \\
593 \\
331 \\
499 \\
749 \\
336 \\
644 \\
872 \\
333 \\
563 \\
950 \\
88 \\
135 \\
762 \\
596 \\
180 \\
359 \\
270 \\
1,158 \\
165 \\
370 \\
146 \\
722 \\
\end{array}$ & $\begin{array}{r}2.1851 \\
2.5601 \\
6.1394 \\
8.0905 \\
3.3004 \\
2.5134 \\
8.4605 \\
3.0228 \\
6.7665 \\
9.3541 \\
1.9218 \\
4.1546 \\
2.8000 \\
5.9990 \\
2.5235 \\
8.3935 \\
12.0278 \\
9.8346 \\
17.4226 \\
11.3592 \\
9.5078 \\
17.8506 \\
9.8228 \\
10.9180 \\
11.0930 \\
5.7948 \\
7.9968 \\
19.3966 \\
5.7431 \\
12.0161 \\
14.4556 \\
6.5232 \\
13.5720 \\
15.8086 \\
1.5364 \\
2.4923 \\
14.9992 \\
12.2004 \\
2.7616 \\
6.9861 \\
4.8988 \\
23.1471 \\
3.3006 \\
7.6690 \\
2.8227 \\
15.3928 \\
\end{array}$ & \begin{tabular}{|l}
0.02512 \\
.01939 \\
.01987 \\
.01972 \\
.01710 \\
.01808 \\
.02110 \\
.01913 \\
.02309 \\
.02093 \\
.02869 \\
.02444 \\
.02258 \\
.01764 \\
.02035 \\
.01756 \\
.02543 \\
.01798 \\
.01846 \\
.01965 \\
.02395 \\
.02062 \\
.01949 \\
.02184 \\
.02205 \\
.01751 \\
.01603 \\
.02590 \\
.01709 \\
.01866 \\
.01658 \\
.01959 \\
.02356 \\
.01664 \\
.01746 \\
.01846 \\
.01968 \\
.02047 \\
.01534 \\
.01946 \\
.01814 \\
.01999 \\
.02001 \\
.02073 \\
.01940 \\
.02132 \\
\end{tabular} & $\begin{array}{r}0.06359 \\
.07450 \\
.18173 \\
.23998 \\
.09670 \\
.07138 \\
.22251 \\
.08522 \\
.18540 \\
.21399 \\
.05631 \\
.11716 \\
.08176 \\
.17637 \\
.07318 \\
.21319 \\
.34524 \\
.26553 \\
.45299 \\
.29079 \\
.24150 \\
.43995 \\
.25834 \\
.23823 \\
.28580 \\
.15470 \\
.22471 \\
.50238 \\
.15679 \\
.30881 \\
.42790 \\
.16373 \\
.34616 \\
.40945 \\
.04164 \\
.06854 \\
.39297 \\
.31842 \\
.07733 \\
.19905 \\
.14060 \\
.63422 \\
.09208 \\
.02017 \\
.03328 \\
.41715\end{array}$ & $\begin{array}{r}0.03387 \\
.05960 \\
.14060 \\
.10194 \\
.06931 \\
.03091 \\
.11760 \\
.05229 \\
.09067 \\
.13470 \\
.03959 \\
.09099 \\
.03304 \\
.04199 \\
.03255 \\
.17458 \\
.21289 \\
.07376 \\
.27528 \\
.21241 \\
.06180 \\
.39272 \\
.20333 \\
.21400 \\
.16529 \\
.10141 \\
.11755 \\
.31229 \\
.12175 \\
.25174 \\
.32236 \\
.12068 \\
.26465 \\
.34937 \\
.03211 \\
.05309 \\
.27898 \\
.20008 \\
.06462 \\
.10828 \\
.13126 \\
.48839 \\
.07261 \\
.16714 \\
.07507 \\
.31248\end{array}$ & $\begin{array}{l}0.0007309 \\
.0005644 \\
.0005881 \\
.0005327 \\
.0005010 \\
.0005135 \\
.0005549 \\
.0005394 \\
.0006475 \\
.0006027 \\
.0008404 \\
.0006892 \\
.0006594 \\
.0005187 \\
.0005902 \\
.0004460 \\
.0007299 \\
.0004877 \\
.0004799 \\
.0005031 \\
.0006225 \\
.0005773 \\
.0005126 \\
.0005765 \\
.0005690 \\
.0004674 \\
.0004503 \\
.0006407 \\
.0000667 \\
.000495 \\
.0004917 \\
.0006149 \\
.0004310 \\
.0004731 \\
.0005077 \\
.0005157 \\
.0005343 \\
.0004296 \\
.0005545 \\
.0005207 \\
.0005464 \\
.0005581 \\
.0005451 \\
.0005704 \\
.0005778\end{array}$ & $\begin{array}{r}0.0003894 \\
.0006787 \\
.0004550 \\
.0002485 \\
.0003591 \\
.0002224 \\
.0002933 \\
.0003309 \\
.0003094 \\
.0003014 \\
.0005910 \\
.0005352 \\
.0002664 \\
.0001235 \\
.0002625 \\
.0003652 \\
.0004501 \\
.0001348 \\
.0002917 \\
.0003675 \\
.0001557 \\
.0004536 \\
.0004034 \\
.0004281 \\
.0002609 \\
.0003064 \\
.0002356 \\
.0004170 \\
.0003622 \\
.0003900 \\
.0003697 \\
.0003624 \\
.0004594 \\
.0003677 \\
.0003649 \\
.0003932 \\
.0003660 \\
.0003557 \\
.0003590 \\
.0003016 \\
.0004861 \\
.0004218 \\
.0004402 \\
.0004519 \\
.0005141 \\
.0004328\end{array}$ \\
\hline Average .. & 2.74 & 1. 79 & 419.3 & 8.2271 & .01991 & .22222 & .14658 & .0005468 & .0003557 \\
\hline
\end{tabular}

3 TO 3.5 PER CENT PROTEID NITROGEN.

\begin{tabular}{|c|c|c|c|c|c|c|c|c|c|}
\hline 20709. & 3.05 & 2.31 & 258 & 5.3229 & 0.02063 & 0.16235 & 0.12296 & 0.0006292 & 0.0004766 \\
\hline $20805 \ldots . . . .$. & 3.32 & 2.26 & 697 & 14. 6942 & .02157 & .48784 & .33208 & .0006999 & .0004875 \\
\hline $21205 \ldots$. & 3. 16 & .22 & 123 & 2. 3642 & .01922 & .07471 & .00520 & .0006074 & .0000423 \\
\hline 21208 . & 3.24 & 2.15 & 287 & 5. 1594 & .01798 & .16712 & .11093 & .0005824 & .0003866 \\
\hline 21307 . & 3.04 & .46 & 143 & 2.5691 & .01796 & .07810 & .01182 & .0005461 & .0000826 \\
\hline 21906 . & 3.18 & 2. 10 & 408 & 10.4800 & .02563 & .33402 & .22008 & .0008168 & .0005382 \\
\hline $21907 \ldots$ & 3.35 & 2.15 & 158 & 2. 9248 & .01851 & .09798 & .06288 & . 0006201 & .0003980 \\
\hline $22206 \ldots$ & 3.22 & 2.11 & 146 & 2. 5712 & .01720 & .08086 & .05425 & .0005538 & .0003629 \\
\hline $22208 \ldots$ & 3.18 & 2.14 & 118 & 1.9090 & .01619 & .06071 & .04084 & .0005144 & .0003465 \\
\hline $22210 \ldots$ & 3.17 & 1.55 & 298 & 6.0173 & .02019 & .19075 & .09327 & .0006401 & .0003129 \\
\hline 22211 . & 3.17 & 1.69 & 561 & 11.5675 & .02062 & .36671 & .19548 & .0006537 & .0003485 \\
\hline $26808 \ldots$ & 3.09 & 2. 28 & 222 & 3.8811 & $.01^{7} 48$ & .11992 & .08849 & .0005402 & .0003985 \\
\hline $28206 \ldots$ & 3.07 & 2.42 & 219 & 4. 3698 & .01996 & .13415 & .10575 & .0006126 & .0004830 \\
\hline $28806 \ldots$ & 3.02 & 1.86 & 685 & 14. 4630 & .02111 & .43679 & .26901 & .0006376 & .0003926 \\
\hline 33305... & 3. 41 & 2.41 & 150 & 3.1346 & .02030 & .10689 & .07554 & .0007126 & .0005037 \\
\hline $33607 \ldots$ & 3. 22 & 2.45 & 136 & 2. 8903 & .02125 & .09307 & .07081 & .0006843 & .0005206 \\
\hline $48306 \ldots$ & 3.29 & 2. 13 & 157 & 2. 6571 & .01692 & .09742 & .05660 & .0005568 & .0003604 \\
\hline $48506 \ldots . . . . .$. & 3.20 & 2.17 & 556 & 9.4585 & .01701 & .30267 & .20525 & .0005444 & .0003691 \\
\hline
\end{tabular}


TABLE 19.-Relation of gliadin-plus-glutenin nitrogen to proteid nitrogen-Continued.

3 TO 3.5 PER CENT PROTEID NITROGEN-Continued.

\begin{tabular}{|c|c|c|c|c|c|c|c|c|c|}
\hline \multirow[b]{2}{*}{ Record number. } & \multicolumn{2}{|c|}{$\begin{array}{c}\text { Percentage } \\
\text { of- }\end{array}$} & \multirow[b]{2}{*}{$\begin{array}{l}\text { Num- } \\
\text { ber of } \\
\text { ker- } \\
\text { nels. }\end{array}$} & \multicolumn{6}{|c|}{ Weight (in grams) of- } \\
\hline & $\begin{array}{c}\text { Pro- } \\
\text { teid } \\
\text { nitro- } \\
\text { gen. }\end{array}$ & $\begin{array}{l}\text { Glia- } \\
\text { din- } \\
\text { plus- } \\
\text { glu- } \\
\text { tenin } \\
\text { nitro- } \\
\text { gen. }\end{array}$ & & $\begin{array}{l}\text { Ker- } \\
\text { nels. }\end{array}$ & $\begin{array}{l}\text { Average } \\
\text { kernel. }\end{array}$ & $\begin{array}{l}\text { Proteid } \\
\text { nitro- } \\
\text { gen in } \\
\text { kernels. }\end{array}$ & $\begin{array}{c}\text { Gliadin- } \\
\text { plus- } \\
\text { glutenin } \\
\text { nitro- } \\
\text { gen in } \\
\text { kernels. }\end{array}$ & $\begin{array}{c}\text { Proteid } \\
\text { nitrogen } \\
\text { in aver- } \\
\text { age ker- } \\
\text { nel. }\end{array}$ & $\begin{array}{l}\text { Gliadin- } \\
\text { plus-glu- } \\
\text { tenin ni- } \\
\text { trogen in } \\
\text { average } \\
\text { kernel. }\end{array}$ \\
\hline 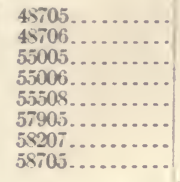 & $\begin{array}{l}3.13 \\
3.00 \\
3.05 \\
3.16 \\
3.11 \\
3.18 \\
3.09 \\
3.01\end{array}$ & $\begin{array}{l}1.56 \\
.71 \\
1.99 \\
1.75 \\
1.96 \\
2.92 \\
2.49 \\
2.47\end{array}$ & $\begin{array}{l}264 \\
379 \\
393 \\
451 \\
216 \\
221 \\
307 \\
235\end{array}$ & $\begin{array}{l}4.3615 \\
6.1983 \\
7.9654 \\
7.1852 \\
3.7407 \\
2.4731 \\
4.2207 \\
2.5436\end{array}$ & $\begin{array}{l}0.01652 \\
.01635 \\
.02028 \\
.01593 \\
.01732 \\
.01118 \\
.01375 \\
.01082\end{array}$ & $\begin{array}{r}0.13652 \\
.18596 \\
.24303 \\
.22705 \\
.11636 \\
.07859 \\
.13042 \\
.07656\end{array}$ & $\begin{array}{l}0.06 \times 04 \\
.04401 \\
.15857 \\
.12574 \\
.07332 \\
.07221 \\
.10510 \\
.06283\end{array}$ & $\begin{array}{l}0.0005171 \\
.0004906 \\
.0006185 \\
.0005034 \\
.0005386 \\
.0003556 \\
.0004248 \\
.0003258\end{array}$ & $\begin{array}{l}0.0002577 \\
.0001161 \\
.0004036 \\
.0002788 \\
.0003395 \\
.0003264 \\
.0003424 \\
.0002673\end{array}$ \\
\hline Average... & 3.16 & 1.95 & 299.5 & 5.5817 & .01817 & .17602 & .10559 & .0005741 & .0003516 \\
\hline
\end{tabular}

3.5 TO 4 PER CENT PROTEID NITROGEN.

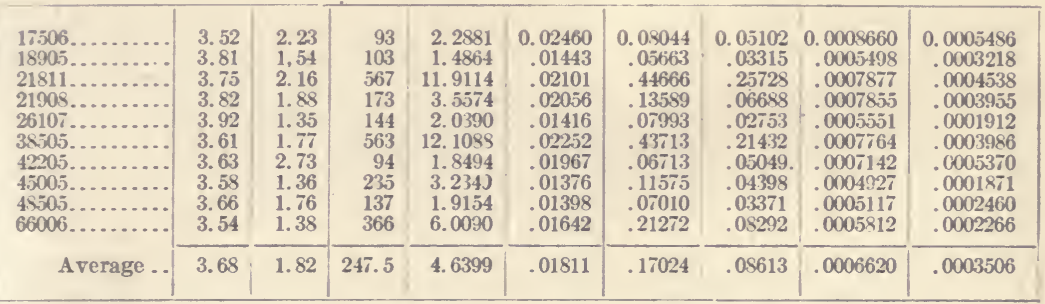

4 TO 4.5 PER CENT PROTEID NITROGEN.

\begin{tabular}{|c|c|c|c|c|c|c|c|c|c|}
\hline $\begin{array}{l}21812 \ldots \ldots \ldots \ldots \\
21813 \ldots \ldots \ldots \ldots \\
21909 \ldots \ldots \ldots \ldots \\
34405 \ldots \ldots \ldots \\
55007 \ldots \ldots \ldots \\
76206 \ldots \ldots \ldots \\
\end{array}$ & $\begin{array}{l}4.26 \\
4.04 \\
4.43 \\
4.33 \\
4.21 \\
4.45\end{array}$ & $\begin{array}{l}2.02 \\
2.14 \\
1.98 \\
2.44 \\
2.21 \\
2.03\end{array}$ & $\begin{array}{l}983 \\
216 \\
525 \\
207 \\
118 \\
447\end{array}$ & $\begin{array}{r}14.8137 \\
4.0258 \\
12.1819 \\
4.1281 \\
2.1571 \\
5.4411\end{array}$ & $\begin{array}{l}0.01507 \\
.01877 \\
.02317 \\
.01994 \\
.01828 \\
.01217\end{array}$ & $\begin{array}{r}0.63107 \\
.16377 \\
.53889 \\
.17875 \\
.09082 \\
.24213\end{array}$ & $\begin{array}{r}0.29934 \\
.08615 \\
.29846 \\
.10073 \\
.04767 \\
.11046\end{array}$ & $\begin{array}{l}0.0006420 \\
.0007582 \\
.0010265 \\
.0008635 \\
.0007696 \\
.0005417\end{array}$ & $\begin{array}{r}0.0003044 \\
.0004017 \\
.0005677 \\
.0004865 \\
.0004040 \\
.0002471\end{array}$ \\
\hline A verage.. & 4. 29 & 2.14 & 416 & 7.1230 & .01790 & .30757 & .15714 & .0007669 & .0004019 \\
\hline
\end{tabular}

MORE THAN 4.5 PER CENT PROTEID NITROGEN.

\begin{tabular}{|c|c|c|c|c|c|c|c|c|c|}
\hline $\begin{array}{l}21206 \ldots \ldots \ldots \\
21210 \ldots \ldots \\
40205 \ldots \ldots \ldots \\
49406 \ldots \ldots \\
69805 \ldots \ldots \\
72607 \ldots \ldots \ldots \\
92306 \ldots \ldots \ldots \\
\end{array}$ & $\begin{array}{l}5.23 \\
5.03 \\
4.69 \\
4.87 \\
5.82 \\
5.59 \\
4.93\end{array}$ & $\begin{array}{l}0.22 \\
1.34 \\
3.07 \\
2.25 \\
1.94 \\
2.01 \\
4.06\end{array}$ & $\begin{array}{l}149 \\
237 \\
194 \\
249 \\
110 \\
188 \\
347\end{array}$ & $\begin{array}{l}2.8564 \\
3.9143 \\
3.6302 \\
3.2964 \\
2.4420 \\
3.4442 \\
6.0091\end{array}$ & $\begin{array}{r}0.01917 \\
.01577 \\
.01871 \\
.01324 \\
.02220 \\
.01832 \\
.01732\end{array}$ & $\begin{array}{r}0.14939 \\
.19639 \\
.17026 \\
.16053 \\
.14213 \\
.19253 \\
.29625\end{array}$ & $\begin{array}{r}0.00628 \\
.05245 \\
.11145 \\
.08168 \\
.04738 \\
.05645 \\
.24397\end{array}$ & $\begin{array}{l}0.0010026 \\
.0007934 \\
.0008776 \\
.0006447 \\
.0012921 \\
.0010241 \\
.0008539\end{array}$ & $\begin{array}{r}0.0000422 \\
.0002113 \\
.0005744 \\
.0002979 \\
.0004307 \\
.0004598 \\
.0007032\end{array}$ \\
\hline Average... & 5.16 & 2.198 & 210.6 & 3.6561 & .01782 & .18685 & .08995 & .0009269 & .0003885 \\
\hline
\end{tabular}


TABLE 20.-Summary of analyses, showing relation of gliadin-plus-glutenin nitrogen to proteid nitrogen.

\begin{tabular}{|c|c|c|c|c|c|c|c|c|c|c|}
\hline \multirow[b]{2}{*}{$\begin{array}{l}\text { Range of per- } \\
\text { centage of } \\
\text { proteid nitro- } \\
\text { gen. }\end{array}$} & \multirow[b]{2}{*}{$\begin{array}{l}\text { Num- } \\
\text { ber of } \\
\text { analy- } \\
\text { ses. }\end{array}$} & \multicolumn{2}{|c|}{$\begin{array}{l}\text { Percentage } \\
\text { of- }\end{array}$} & \multirow[b]{2}{*}{$\begin{array}{l}\text { Num- } \\
\text { ber of } \\
\text { ker- } \\
\text { nels. }\end{array}$} & \multicolumn{6}{|c|}{ Weight (in grams) of- } \\
\hline & & $\begin{array}{l}\text { Pro- } \\
\text { teid } \\
\text { nitro- } \\
\text { gen. }\end{array}$ & $\begin{array}{l}\text { Glia- } \\
\text { din- } \\
\text { plus- } \\
\text { glu- } \\
\text { tenin } \\
\text { nitro- } \\
\text { gen. }\end{array}$ & & Kernels. & $\begin{array}{l}\text { Average } \\
\text { kernel. }\end{array}$ & $\begin{array}{c}\text { Proteid } \\
\text { nitrogen } \\
\text { in ker- } \\
\text { nels. }\end{array}$ & $\begin{array}{l}\text { Gliadin- } \\
\text { plus-glu- } \\
\text { tenin } \\
\text { nitrogen } \\
\text { in } \\
\text { kernels. }\end{array}$ & $\begin{array}{c}\text { Proteid } \\
\text { nitrogen } \\
\text { in } \\
\text { average } \\
\text { kernel. }\end{array}$ & $\begin{array}{l}\text { Gliadin- } \\
\text { plus-glu- } \\
\text { tenin ni- } \\
\text { trogen in } \\
\text { average } \\
\text { kernel. }\end{array}$ \\
\hline $\begin{array}{l}1 \text { to } 2 \ldots \ldots \ldots \\
2 \text { to } 2.5 \ldots \ldots \ldots \\
2.5 \text { to } 3 \ldots \ldots \ldots \ldots \\
3 \text { to } 3.5 \ldots \ldots \ldots \\
3.5 \text { to } 4 \ldots \ldots \ldots \\
4 \text { to } 4.5 \ldots \ldots \ldots \ldots \\
4.5 \text { and over..... }\end{array}$ & $\begin{array}{r}3 \\
21 \\
70 \\
26 \\
10 \\
6 \\
7\end{array}$ & $\begin{array}{l}1.89 \\
2.30 \\
2.74 \\
3.16 \\
3.68 \\
4.29 \\
5.16\end{array}$ & $\begin{array}{l}1.76 \\
1.68 \\
1.73 \\
1.95 \\
1.82 \\
2.22 \\
2.20\end{array}$ & $\begin{array}{l}512.0 \\
489.6 \\
419.3 \\
299.5 \\
247.5 \\
416.0 \\
210.6\end{array}$ & $\begin{array}{r}10.4207 \\
10.5874 \\
8.2271 \\
5.5817 \\
4.6399 \\
7.1230 \\
3.6561\end{array}$ & $\begin{array}{r}0.01978 \\
.02173 \\
.01991 \\
.01817 \\
.01811 \\
.01790 \\
.01782\end{array}$ & $\begin{array}{l}0.19568 \\
.24272 \\
.22222 \\
.17602 \\
.17024 \\
.30757 \\
.18685\end{array}$ & $\begin{array}{r}0.18667 \\
.17872 \\
.13948 \\
.10889 \\
.08613 \\
.15714 \\
.08995\end{array}$ & $\begin{array}{r}0.0003744 \\
.0004991 \\
.0005468 \\
.0005741 \\
.0006620 \\
.0007669 \\
.0009269\end{array}$ & $\begin{array}{r}0.0003518 \\
.0003652 \\
.0003442 \\
.0003516 \\
.0003506 \\
.0004019 \\
.0003886\end{array}$ \\
\hline
\end{tabular}

The figures in Table 20 show that while gliadin-plus-glutenin nitrogen increases with proteid nitrogen it does not do so in the same ratio, the increase in proteid nitrogen being due in large measure to an increase in other proteids.

The same analyses are tabulated in Table 21 according to the increase in gliadin-plus-glutenin nitrogen, and the averages for each group are stated in Table 22 . In the latter table the increase in proteid nitrogen does not keep pace with the increase in gliadin-plusglutenin nitrogen, there being 1.74 per cent other proteid nitrogen in the first group and 1.25 per cent in the last.

It thus becomes evident that a determination of proteid nitrogen in the kernel is not an accurate guide to the content of gliadin plus glutenin, and that a direct determination of these substances is necessary.

It is, furthermore, apparent that a determination of gliadin-plusglutenin nitrogen will permit of the selection of kernels having a large percentage of these substances.

TABLE 21.-Relation of proteid nitrogen to gliadin-plus-glutenin nitrogen.

GLIADIN-PLUS-GLUTENIN NITROGEN, 1 TO 1.5 PER CENT.

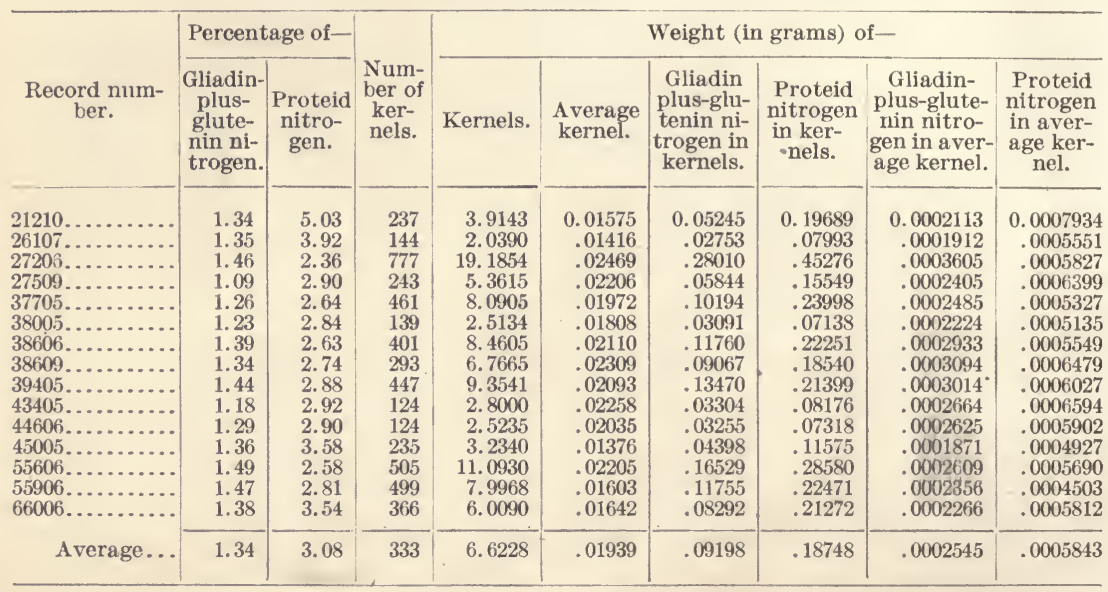


TABLE 21.-Relation of proteid nitrogen to gliadin-plus-glutenin nitrogen-Continued.

GLIADIN-PLUS-GLUTENIN NITROGEN, 1.5 TO 2 PER CENT.

\begin{tabular}{|c|c|c|c|c|c|c|c|c|c|}
\hline \multirow[b]{2}{*}{$\begin{array}{l}\text { Record num- } \\
\text { ter. }\end{array}$} & \multicolumn{2}{|c|}{ Percentage of- } & \multirow[b]{2}{*}{$\begin{array}{c}\text { Num- } \\
\text { ber of } \\
\text { ker- } \\
\text { nels. }\end{array}$} & \multicolumn{6}{|c|}{ Weight (in grams) of- } \\
\hline & $\begin{array}{c}\text { Gliadin- } \\
\text { plus- } \\
\text { glute- } \\
\text { nin ni- } \\
\text { trogen. }\end{array}$ & $\begin{array}{c}\text { Proteid } \\
\text { nitro- } \\
\text { gen. }\end{array}$ & & Kernels. & $\begin{array}{l}\text { Average } \\
\text { kernel. }\end{array}$ & $\begin{array}{l}\text { Gliadin- } \\
\text { plus-glu- } \\
\text { tenin ni- } \\
\text { trogen in } \\
\text { kernels. }\end{array}$ & $\begin{array}{c}\text { Proteid } \\
\text { nitrogen } \\
\text { in ker- } \\
\text { nels. }\end{array}$ & $\begin{array}{l}\text { Gliadin- } \\
\text { plus-glute- } \\
\text { nin nitro- } \\
\text { gen in aver- } \\
\text { age kernel. }\end{array}$ & $\begin{array}{c}\text { Proteid } \\
\text { nitrogen } \\
\text { in aver- } \\
\text { age ker- } \\
\text { nel. }\end{array}$ \\
\hline $\begin{array}{r}808 \ldots \\
908 \ldots \\
909 \\
205 \\
207 \\
211 \\
211 \ldots \\
906 \ldots \\
909 \\
905\end{array}$ & $\begin{array}{l}1.54 \\
1.85 \\
1.97 \\
1.96 \\
1.98 \\
1.97 \\
1.82 \\
1.55 \\
1.69 \\
1.82 \\
1.88 \\
1.90 \\
1.70 \\
1.95 \\
1.73 \\
1.65 \\
1.98 \\
1.55 \\
1.96 \\
1.77 \\
1.73 \\
1.84 \\
1.80 \\
1.77 \\
1.50 \\
1.76 \\
1.56 \\
1.99 \\
1.75 \\
1.58 \\
1.87 \\
1.97 \\
1.56 \\
1.96 \\
1.96 \\
1.75 \\
1.61 \\
1.96 \\
1.66 \\
1.85 \\
1.95 \\
1.83 \\
1.95 \\
1.86 \\
1.55 \\
1.68 \\
1.81 \\
1.95 \\
1.94 \\
1.76 \\
1.96 \\
\end{array}$ & $\begin{array}{l}3.81 \\
2.77 \\
2.67 \\
2.57 \\
3.82 \\
4.43 \\
2.81 \\
2.77 \\
3.17 \\
3.17 \\
2.71 \\
2.80 \\
2.63 \\
2.41 \\
2.92 \\
2.58 \\
2.12 \\
2.70 \\
2.91 \\
3.02 \\
2.39 \\
3.61 \\
2.82 \\
2.11 \\
2.38 \\
2.87 \\
2.02 \\
3.66 \\
3.13 \\
3.05 \\
3.16 \\
2.60 \\
2.57 \\
2.48 \\
1.89 \\
3.11 \\
2.64 \\
2.67 \\
2.59 \\
2.42 \\
2.30 \\
2.51 \\
2.42 \\
2.34 \\
2.61 \\
2.62 \\
2.61 \\
2.85 \\
2.41 \\
2.28 \\
2.09 \\
5.82 \\
1.81 \\
1.98 \\
2.41\end{array}$ & $\begin{array}{r}103 \\
444 \\
312 \\
1,156 \\
173 \\
525 \\
283 \\
169 \\
298 \\
561 \\
228 \\
180 \\
866 \\
891 \\
166 \\
267 \\
539 \\
444 \\
87 \\
685 \\
301 \\
563 \\
158 \\
1,031 \\
608 \\
473 \\
314 \\
137 \\
264 \\
393 \\
451 \\
944 \\
578 \\
167 \\
342 \\
216 \\
500 \\
331 \\
749 \\
562 \\
302 \\
333 \\
509 \\
462 \\
563 \\
762 \\
596 \\
359 \\
544 \\
373 \\
583 \\
110 \\
729 \\
465 \\
287 \\
\end{array}$ & $\begin{array}{r}1.4864 \\
9.9070 \\
6.2514 \\
19.7446 \\
3.5574 \\
12.1819 \\
2.6965 \\
3.2787 \\
6.0173 \\
11.5675 \\
4.2376 \\
2.9999 \\
16.4120 \\
16.4061 \\
3.3266 \\
5.5666 \\
12.0399 \\
10.0005 \\
2.1851 \\
14.4630 \\
7.0596 \\
12.1088 \\
3.0228 \\
21.5399 \\
11.6655 \\
12.0278 \\
6.4302 \\
1.9154 \\
4.3615 \\
7.9684 \\
7.1852 \\
17.4226 \\
11.3592 \\
2.5160 \\
5.6864 \\
3.7407 \\
10.9180 \\
5.7948 \\
19.3966 \\
12.2210 \\
9.2120 \\
6.5232 \\
9.3093 \\
10.9073 \\
13.5720 \\
14.9992 \\
12.2004 \\
6.9861 \\
9.8298 \\
7.0051 \\
11.7066 \\
2.4420 \\
15.7835 \\
9.7922 \\
7.3993\end{array}$ & $\begin{array}{r}0.01443 \\
.02282 \\
.02004 \\
.01708 \\
.02056 \\
.02317 \\
.00953 \\
.01940 \\
.02019 \\
.02062 \\
.01859 \\
.01667 \\
.01895 \\
.01841 \\
.02004 \\
.02085 \\
.02183 \\
.02252 \\
.02572 \\
.02111 \\
.02345 \\
.02252 \\
.01913 \\
.02089 \\
.01919 \\
.02543 \\
.02048 \\
.01398 \\
.01652 \\
.02028 \\
.01593 \\
.01846 \\
.01965 \\
.01507 \\
.01663 \\
.01732 \\
.02184 \\
.01751 \\
.02590 \\
.02175 \\
.03050 \\
.01959 \\
.01829 \\
.03361 \\
.02356 \\
.01968 \\
.02047 \\
.01946 \\
.01807 \\
.01878 \\
.02008 \\
.02220 \\
.02165 \\
.02106 \\
.02578\end{array}$ & $\begin{array}{r}0.03315 \\
.18328 \\
.12315 \\
.38700 \\
.06688 \\
.29846 \\
.05312 \\
.05967 \\
.09327 \\
.19548 \\
.07712 \\
.05640 \\
.31182 \\
.27890 \\
.06487 \\
.09630 \\
.19866 \\
.19800 \\
.03887 \\
.26901 \\
.13554 \\
.21432 \\
.05229 \\
.39635 \\
.20997 \\
.21289 \\
.09645 \\
.03371 \\
.06804 \\
.15857 \\
.12574 \\
.27528 \\
.21241 \\
.04957 \\
.08871 \\
.07332 \\
.21400 \\
.10141 \\
.31229 \\
.23953 \\
.15292 \\
.12068 \\
.18153 \\
.19960 \\
.26465 \\
.27898 \\
.20008 \\
.10828 \\
.16514 \\
.12680 \\
.22828 \\
.04738 \\
.0727\end{array}$ & $\begin{array}{r}0.05663 \\
.27443 \\
.16691 \\
.50744 \\
.13589 \\
.53889 \\
.07577 \\
.09082 \\
.19075 \\
.36671 \\
.11484 \\
.08400 \\
.43164 \\
.39539 \\
.09712 \\
.14362 \\
.24942 \\
.27003 \\
.06359 \\
.43679 \\
.16872 \\
.43713 \\
.08522 \\
.45435 \\
.27765 \\
.34524 \\
.12989 \\
.07010 \\
.13652 \\
.24303 \\
.22705 \\
.45299 \\
.29079 \\
.06240 \\
.10747 \\
.11636 \\
.28823 \\
.15470 \\
.50238 \\
.29575 \\
.21187 \\
.16373 \\
.22529 \\
.25522 \\
.34616 \\
.39297 \\
.31842 \\
.19905 \\
.23690 \\
.15971 \\
.24468 \\
.14213 \\
.28569 \\
.19388 \\
.17833\end{array}$ & $\begin{array}{c}0.0003218 \\
.0004222 \\
.0003948 \\
.0003348 \\
.0003955 \\
.0005677 \\
.0001877 \\
.0003531 \\
.0003129 \\
.0003485 \\
.0003383 \\
.0003134 \\
.0003600 \\
.0003130 \\
.0003908 \\
.0003607 \\
.0003602 \\
.0004459 \\
.0003894 \\
.0003926 \\
.0001502 \\
.0003986 \\
.0003309 \\
.0003444 \\
.0004501 \\
.0003072 \\
.0002460 \\
.0002577 \\
.0004036 \\
.0002788 \\
.0002917 \\
.0003675 \\
.0002969 \\
.0002599 \\
.0003395 \\
.0004281 \\
.0003064 \\
.0004170 \\
.0004263 \\
.0005063 \\
.0003624 \\
.0003566 \\
.0004594 \\
.0003640 \\
.0003357 \\
.0003016 \\
.0003036 \\
.0003399 \\
.0003916 \\
.0004307 \\
.0003832 \\
.0004228 \\
\end{array}$ & $\begin{array}{r}0.0005498 \\
.0006181 \\
.0005350 \\
.0004389 \\
.0007855 \\
.0010265 \\
.0002677 \\
.0005376 \\
.0006401 \\
.0006537 \\
.0005037 \\
.0004667 \\
.0004984 \\
.0004437 \\
.0005850 \\
.0005379 \\
.0004627 \\
.0006082 \\
.0007309 \\
.0006376 \\
.0005605 \\
.0007764 \\
.0005394 \\
.0004407 \\
.0004567 \\
.0007299\end{array}$ \\
\hline A verag & .80 & 2.76 & 2.5 & 9.0243 & .02016 & .16392 & .23801 & .0003653 & .000553 \\
\hline
\end{tabular}

GLIADIN-PLUS-GLUTENIN NITROGEN, 2 TO 2.5 PER CENT.

\begin{tabular}{|c|c|c|c|c|c|c|c|c|c|}
\hline $\begin{array}{l}17506 \ldots \ldots \ldots \\
20706 \ldots \ldots \ldots \\
20709 \ldots \ldots \ldots \\
20710 \ldots \ldots \\
20805 \ldots \ldots \ldots \\
21208 \ldots \ldots \ldots \\
21807 \ldots \ldots \ldots \\
21809 \ldots \ldots \ldots \\
21811 \ldots \ldots \ldots \\
21812 \ldots \ldots \ldots \\
21813 \ldots \ldots \ldots \\
21905 \ldots \ldots \ldots \\
\end{array}$ & $\begin{array}{l}2.23 \\
2.05 \\
2.31 \\
2.00 \\
2.26 \\
2.15 \\
2.11 \\
2.18 \\
2.16 \\
2.02 \\
2.14 \\
2.18\end{array}$ & $\begin{array}{l}3.52 \\
2.78 \\
3.05 \\
2.83 \\
3.32 \\
3.24 \\
2.73 \\
2.73 \\
3.75 \\
4.26 \\
4.04 \\
2.64\end{array}$ & \begin{tabular}{r|}
93 \\
163 \\
258 \\
867 \\
697 \\
287 \\
377 \\
418 \\
567 \\
983 \\
216 \\
791
\end{tabular} & $\begin{array}{r}2.2581 \\
3.3138 \\
5.3229 \\
17.1115 \\
14.6942 \\
5.1594 \\
9.4172 \\
8.0214 \\
11.9114 \\
14.8139 \\
4.0258 \\
14.3111\end{array}$ & $\begin{array}{l}0.02450 \\
.02033 \\
.02063 \\
.01974 \\
.02157 \\
.01798 \\
.02498 \\
.01919 \\
.02101 \\
.01507 \\
.01877 \\
.01809\end{array}$ & $\begin{array}{l}0.05102 \\
.06793 \\
.12296 \\
.34222 \\
.33208 \\
.11093 \\
.19870 \\
.17487 \\
.25728 \\
.29934 \\
.08615 \\
.31198\end{array}$ & $\begin{array}{r}0.08044 \\
.09212 \\
.16235 \\
.48428 \\
.48784 \\
.16712 \\
.25709 \\
.21898 \\
.44666 \\
.63107 \\
.16377 \\
.37781\end{array}$ & $\begin{array}{r}0.0005486 \\
.0004168 \\
.0004766 \\
.0003948 \\
.0004875 \\
.000386 .6 \\
.0005271 \\
.0004183 \\
.0004538 \\
.0003044 \\
.0004017 \\
.0003944\end{array}$ & $\begin{array}{r}0.0008660 \\
.0005652 \\
.0006282 \\
.0005586 \\
.0006999 \\
.0005824 \\
.0006664 \\
.0005238 \\
.0007877 \\
.0006420 \\
.0007582 \\
.0004777\end{array}$ \\
\hline
\end{tabular}


TABLE 21.-Relation of proteid nitrogen to gliadin-plus-glutenin nitrogen-Continued.

GLIADIN-PLUS-GLUTENIN NITROGEN, 2 TO 2.5 PER CENT-Continued.

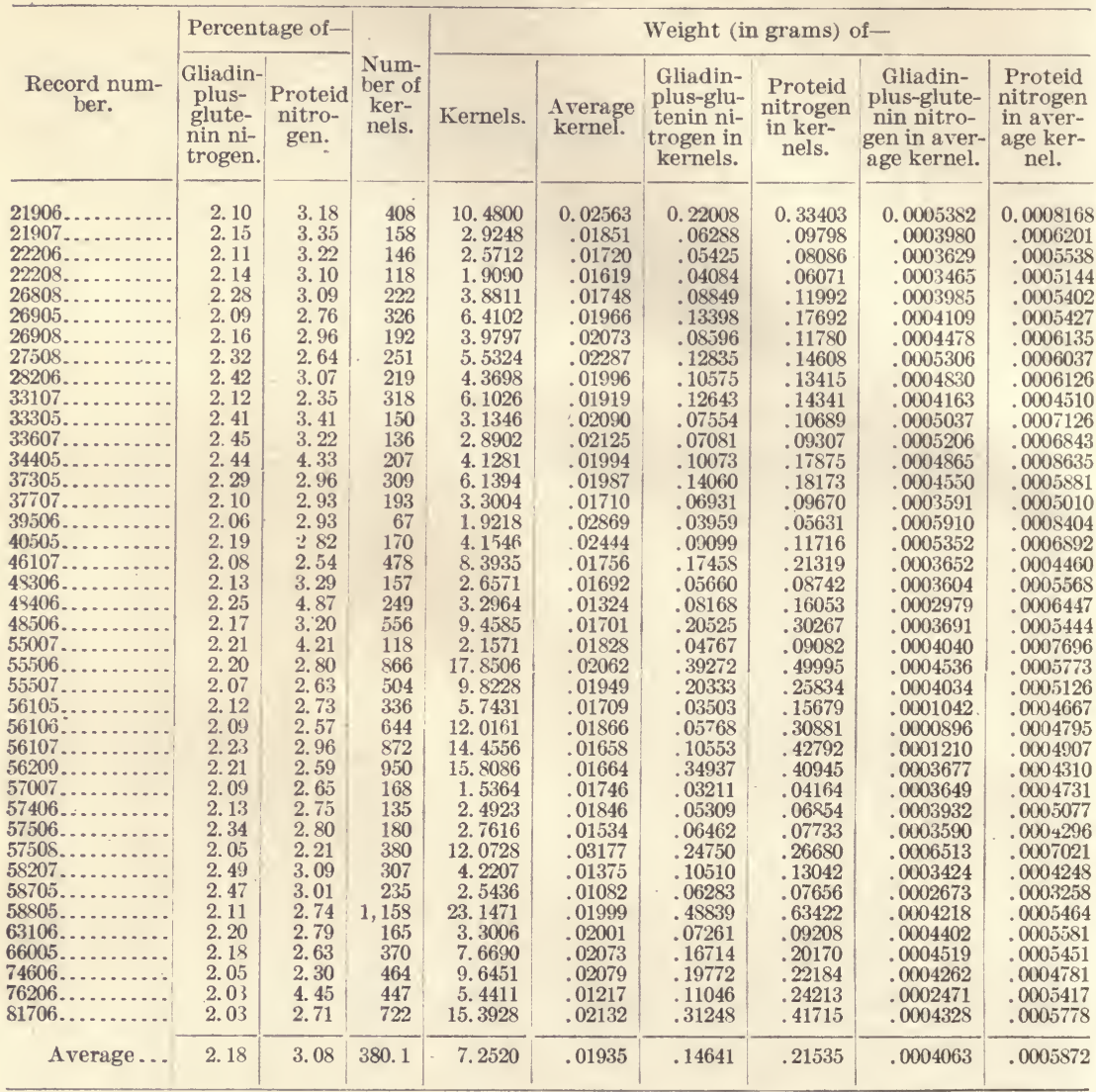

GLIADIN-PLUS-GLUTENIN NITROGEN, 2.5 TO 3 PER CENT.

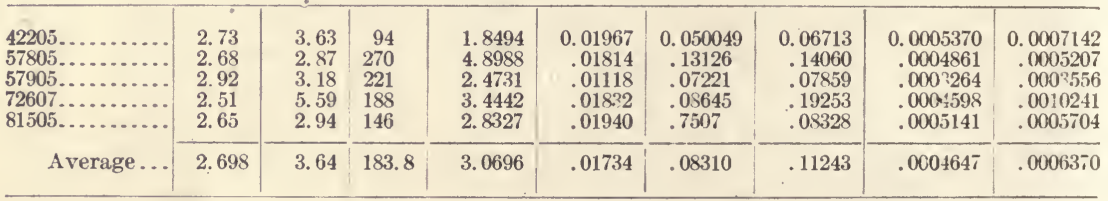

GLIADIN-PLUS-GLUTENIN NITROGEN, 3 PER CENT AND, OVER.

\begin{tabular}{|c|c|c|c|c|c|c|c|c|c|}
\hline $\begin{array}{l}40205 \ldots \ldots \ldots \ldots \\
92306 \ldots \ldots \ldots \ldots\end{array}$ & $\begin{array}{l}3.07 \\
4.06\end{array}$ & $\begin{array}{l}4.69 \\
4.93\end{array}$ & $\begin{array}{l}194 \\
347\end{array}$ & $\begin{array}{l}\text { 3. } 6302 \\
6.0091\end{array}$ & $\begin{array}{r}0.01871 \\
.01732\end{array}$ & $\begin{array}{r}0.11145 \\
.24397\end{array}$ & $\begin{array}{r}0.17026 \\
.29625\end{array}$ & $\begin{array}{r}0.0005744 \\
.0007032\end{array}$ & $\begin{array}{r}0.0008776 \\
.0008539\end{array}$ \\
\hline Average... & 3.56 & 4. 81 & 270.5 & 4. 8196 & .01801 & .17771 & .23325 & .0006388 & .0008657 \\
\hline
\end{tabular}


TABLE 22.-Summary of analyses, showing relation of proteid nitrogen to gliadin-pl:sglutenin nitrogen.

\begin{tabular}{|c|c|c|c|c|c|c|c|c|c|c|}
\hline \multirow[b]{2}{*}{$\begin{array}{l}\text { Range of } \\
\text { percentage of } \\
\text { gliadin-plus- } \\
\text { glutenin ni- } \\
\text { trogen. }\end{array}$} & \multicolumn{2}{|c|}{$\begin{array}{c}\text { Percentage } \\
\text { of }-\end{array}$} & \multicolumn{2}{|c|}{$\begin{array}{l}\text { Number } \\
\text { of- }\end{array}$} & & \multicolumn{4}{|c|}{ Weight (in grams) of- } \\
\hline & $\begin{array}{l}\text { Glia- } \\
\text { din- } \\
\text { plus- } \\
\text { glu- } \\
\text { tenin } \\
\text { nitro- } \\
\text { gen. }\end{array}$ & $\begin{array}{c}\text { Pro- } \\
\text { teid } \\
\text { nitro- } \\
\text { gen. }\end{array}$ & $\begin{array}{l}\text { An- } \\
\text { aly- } \\
\text { ses. }\end{array}$ & $\begin{array}{l}\text { Ker- } \\
\text { nels. }\end{array}$ & Kernels. & $\begin{array}{l}\text { A verage } \\
\text { kernel. }\end{array}$ & $\begin{array}{l}\text { Gliadin- } \\
\text { plus-glu- } \\
\text { tenin ni- } \\
\text { trogen } \\
\text { in ker- } \\
\text { nels. }\end{array}$ & $\begin{array}{c}\text { Proteid } \\
\text { nitrogen } \\
\text { in ker- } \\
\text { nels. }\end{array}$ & $\begin{array}{l}\text { Gliadin- } \\
\text { plus-glute- } \\
\text { nin nitro- } \\
\text { gen in } \\
\text { average } \\
\text { kernel. }\end{array}$ & $\begin{array}{l}\text { Proteid } \\
\text { nitrogen } \\
\text { in a ver- } \\
\text { age ker- } \\
\text { nel. }\end{array}$ \\
\hline $\begin{array}{l}1 \text { to } 1.5 \ldots \ldots \\
1.5 \text { to } 2 \ldots \ldots \ldots \\
2 \text { to } 2.5 \ldots \ldots \\
2.5 \text { to } 3 \ldots \ldots \\
3 \text { and over.... }\end{array}$ & \begin{tabular}{|l|}
1.34 \\
1.80 \\
2.18 \\
2.70 \\
3.56
\end{tabular} & $\begin{array}{l}3.08 \\
2.76 \\
3.08 \\
3.64 \\
4.81\end{array}$ & $\begin{array}{r}15 \\
55 \\
52 \\
5 \\
2\end{array}$ & $\begin{array}{l}333 \\
442.5 \\
380.1 \\
183.8 \\
270.5\end{array}$ & $\begin{array}{l}6.6228 \\
9.0243 \\
7.2520 \\
3.0696 \\
4.8196\end{array}$ & $\begin{array}{l}0.01939 \\
.02016 \\
.01935 \\
.01734 \\
.01801\end{array}$ & $\begin{array}{r}0.09198 \\
.16392 \\
.14641 \\
.08310 \\
.17771\end{array}$ & $\begin{array}{l}0.18748 \\
.23801 \\
.21535 \\
.11243 \\
.23325\end{array}$ & $\begin{array}{r}0.0002545 \\
.0003653 \\
.0004063 \\
.0004647 \\
.0006388\end{array}$ & $\begin{array}{r}0.0005843 \\
.0005528 \\
.00058 ; 2 \\
.0006370 \\
.0005657\end{array}$ \\
\hline
\end{tabular}

IMPROVEMENT IN THE QUALITY OF THE GLUTEN.

It is well known that large differences exist in the bread-making values of different varieties of wheats eren when they have approximately the same gluten content and are raised in the same locality. This fact is generally attributed to differences in the quality of the gluten.

W. Farrar ${ }^{a}$ points out the difference in the bread-making qualities of two wheats due to the quality of the gluten. He compares Saxon Fife wheat, which had a gluten content of 9.92 per cent, and which produced 309 pounds of bread from 200 pounds of flour, with Purple Straw Tuscan wheat, which had a gluten content of 9.94 per cent, and which produced only 278 pounds of bread from the same quantity of flour.

In this case it was not the amount but the quality of the gluten that determined the greater excellence of the Saxon Fife wheat.

It has further been stated by Girard, ${ }^{b}$ Snyder, ${ }^{c}$ and Guthrie ${ }^{d}$ that the ratio in which gliadin and glutenin exist in the gluten determines its value for bread making.

It was considered desirable to ascertain whether the proportions of these two constituents remain about the same in wheats of high and of low content. If the quality of the gluten remains constant as the quantity increases, the value of the wheat for bread making will improve in about the same ratio. If, on the other hand, there is a tendency for the quality to deteriorate as the quantity increases, there would be greater difficulty in effecting improvement.

In Table 23, analyses of the crop of 1903 are arranged in groups according to their content of gliadin plus glutenin. The first group comprises all plants having less than 1 per cent, and each succeeding group increases by 0.25 per cent. It is followed by Table 24 , which is a summary of Table 23.

a Agricultural Gazette of New South Wales, 9 (1898), pp. 241-250.

${ }^{b}$ Compt. Rend., 1897, p. 876.

c Minnesota Experiment Station Bulletins 54 and 63.

$d$ Agrieultural Gazette of New South Wales, 9 (1898), pp. 363-374. 
TABLE 23.-Ratio of gliadin to glutenin as the content of their sum increases.

GLIADIN-PLUS-GLUTENIN NITROGEN, BELOW 1 PER CENT.

\begin{tabular}{|c|c|c|c|c|c|c|c|}
\hline \multirow[b]{2}{*}{ Record number. } & \multicolumn{3}{|c|}{ Percentage of- } & \multicolumn{2}{|c|}{ Proportion of- } & \multicolumn{2}{|c|}{ Percentage of- } \\
\hline & $\begin{array}{l}\text { Gliadin- } \\
\text { plus- } \\
\text { glutenin } \\
\text { nitrogen. }\end{array}$ & $\begin{array}{l}\text { Gliadin } \\
\text { nitrogen. }\end{array}$ & $\begin{array}{l}\text { Glutenin } \\
\text { nitrogen. }\end{array}$ & Gliadin. & Glutenin. & $\begin{array}{l}\text { Proteid } \\
\text { nitrogen. }\end{array}$ & $\begin{array}{c}\text { Other } \\
\text { proteid } \\
\text { nitrogen }\end{array}$ \\
\hline $\begin{array}{l}21205 \ldots \ldots \ldots \\
21206 \ldots \ldots \ldots \\
21207 \ldots \ldots \ldots \\
21212 \ldots \ldots \\
21306 \ldots \ldots \\
21307 \ldots \ldots \ldots \\
21805 \ldots \ldots \ldots \\
27307 \ldots \ldots \ldots \\
48806 \ldots \ldots \ldots \\
55308 \ldots \ldots \ldots \\
81707 \ldots \ldots \ldots \\
\end{array}$ & $\begin{array}{l}0.216 \\
.218 \\
.170 \\
.192 \\
.975 \\
.461 \\
.230 \\
.821 \\
.748 \\
.655 \\
.636\end{array}$ & $\begin{array}{r}0.114 \\
.142 \\
.099 \\
.109 \\
.505 \\
.255 \\
.126 \\
.806 \\
.018 \\
.629 \\
.237\end{array}$ & $\begin{array}{l}0.102 \\
.076 \\
.071 \\
.083 \\
.470 \\
.206 \\
.104 \\
.015 \\
.730 \\
.026 \\
.399\end{array}$ & $\begin{array}{l}0.528 \\
.651 \\
.582 \\
.567 \\
.518 \\
.447 \\
.548 \\
.982 \\
.024 \\
.960 \\
.372\end{array}$ & $\begin{array}{l}0.472 \\
.349 \\
.418 \\
.433 \\
.482 \\
.553 \\
.452 \\
.018 \\
.976 \\
.040 \\
.628\end{array}$ & $\begin{array}{l}3.16 \\
5.23 \\
2.96 \\
2.16 \\
2.90 \\
3.04 \\
2.69 \\
2.53 \\
2.70 \\
2.54 \\
2.34\end{array}$ & $\begin{array}{l}2.944 \\
5.012 \\
2.790 \\
1.968 \\
1.925 \\
2.579 \\
2.460 \\
1.709 \\
1.952 \\
1.885 \\
1.704\end{array}$ \\
\hline Average... & .484 & .276 & .208 & .562 & .438 & 2.93 & 2.448 \\
\hline
\end{tabular}

GI.IADIN-PLUS-GLUTENIN NITROGEN, 1 TO 1.25 PER CENT.

\begin{tabular}{|c|c|c|c|c|c|c|c|}
\hline $\begin{array}{l}27509 \ldots \ldots \ldots \\
38005 \ldots \ldots \ldots \ldots \\
43405 \ldots \ldots \ldots \ldots\end{array}$ & $\begin{array}{l}1.087 \\
1.227 \\
1.184\end{array}$ & $\begin{array}{r}1.072 \\
.593 \\
1.078\end{array}$ & $\begin{array}{r}0.015 \\
.634 \\
.106\end{array}$ & $\begin{array}{r}0.986 \\
.483 \\
.910\end{array}$ & $\begin{array}{r}0.014 \\
.517 \\
.090\end{array}$ & $\begin{array}{l}2.90 \\
2.84 \\
2.92\end{array}$ & $\begin{array}{l}1.813 \\
1.613 \\
1.736\end{array}$ \\
\hline Average... & 1.166 & .914 & .252 & .793 & .207 & 2.89 & 1.721 \\
\hline
\end{tabular}

GLIADIN-PLUS-GLUTENIN NITROGEN, 1.25 TO 1.50 PER CENT.

\begin{tabular}{|c|c|c|c|c|c|c|c|}
\hline 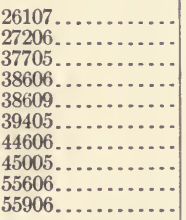 & $\begin{array}{l}1.352 \\
1.465 \\
1.265 \\
1.387 \\
1.336 \\
1.439 \\
1.287 \\
1.361 \\
1.493 \\
1.470\end{array}$ & $\begin{array}{r}0.108 \\
.815 \\
.715 \\
.725 \\
.586 \\
.818 \\
1.057 \\
1.240 \\
.899 \\
.443\end{array}$ & $\begin{array}{r}1.244 \\
.650 \\
.550 \\
.662 \\
.750 \\
.621 \\
.230 \\
.121 \\
.594 \\
1.027\end{array}$ & $\begin{array}{r}0.080 \\
.556 \\
.565 \\
.522 \\
.439 \\
.568 \\
.821 \\
.911 \\
.602 \\
.301\end{array}$ & $\begin{array}{r}0.920 \\
. \quad .444 \\
.435 \\
.478 \\
.561 \\
.432 \\
.179 \\
.089 \\
.398 \\
.699\end{array}$ & $\begin{array}{l}3.92 \\
2.36 \\
2.64 \\
2.63 \\
2.74 \\
2.88 \\
2.90 \\
3.58 \\
2.58 \\
2.81\end{array}$ & $\begin{array}{l}2.568 \\
.895 \\
1.375 \\
1.243 \\
1.404 \\
1.441 \\
1.613 \\
2.219 \\
1.087 \\
1.340\end{array}$ \\
\hline Average... & 1.385 & .741 & .645 & .536 & .463 & 2.90 & 1.518 \\
\hline
\end{tabular}

GLIADIN-PLUS-GLUTENIN NITROGEN, 1.50 TO 1.75 PER CENT.

\begin{tabular}{|c|c|c|c|c|c|c|c|}
\hline $18905 \ldots \ldots \ldots$ & 1.537 & 0.143 & 1.394 & 0.093 & 0.907 & 3.81 & 2.273 \\
\hline $22210 \ldots \ldots$ & 1.555 & .801 & .754 & .515 & .485 & 3.17 & 1.615 \\
\hline $22211 \ldots \ldots \ldots \ldots$ & 1.692 & 1.002 & .690 & .592 & .408 & 3.17 & 1.478 \\
\hline $27205 \ldots \ldots \ldots$ & 1.700 & 1.073 & .627 & .631 & .369 & 2.41 & .710 \\
\hline $27305 \ldots \ldots$ & 1.735 & 1.075 & .660 & .619 & .381 & 2.58 & .845 \\
\hline $27505 \ldots . . . . .$. & 1.651 & 1.032 & .619 & .625 & .375 & 2.12 & .469 \\
\hline $28805 \ldots . . . . . .$. & 1.555 & .958 & .597 & .616 & .384 & 2.91 & 1.355 \\
\hline $38608 \ldots . . \ldots \ldots . . .$. & 1. 731 & .962 & .769 & .556 & .444 & 2.82 & 1.089 \\
\hline $48409 \ldots \ldots \ldots \ldots$ & 1.504 & .690 & .814 & .459 & .541 & 2.02 & .516 \\
\hline $48705 \ldots \ldots \ldots$. & 1.563 & .057 & 1.506 & .036 & .964 & 3.13 & 1.567 \\
\hline $55008 \ldots \ldots \ldots . . .$. & 1.581 & .687 & .894 & .435 & .565 & 2.60 & 1.019 \\
\hline $55307 \ldots \ldots \ldots \ldots$ & 1.561 & .908 & .653 & .582 & .418 & 1.89 & .329 \\
\hline $55907 \ldots \ldots$ & 1. 608 & .632 & .976 & .393 & .607 & 2. 59 & .982 \\
\hline 55909 & 1.658 & .810 & .848 & .488 & .512 & 2.30 & .642 \\
\hline $57408 \ldots \ldots \ldots$ & 1.639 & 1.177 & .462 & .717 & .283 & 2.61 & .971 \\
\hline $57507 \ldots \ldots \ldots$ & 1.546 & 1. 141 & .405 & .738 & .262 & 2.85 & 1.304 \\
\hline $65306 \ldots \ldots$ & 1.683 & .965 & .718 & .573 & .427 & 2.41 & .727 \\
\hline $81708 \ldots . . . . .$. & 1. 641 & 1.221 & .420 & .744 & .256 & 2.41 & .769 \\
\hline Average... & 1.619 & .852 &. .767 & .523 & .477 & 2.65 & 1.037 \\
\hline
\end{tabular}


TABLE 23.-Ratio of gliadin to glutenin as the content of their sum increases-Continued.

GLIADIN-PLUS-GLUTENIN NITROGEN, 1.75 TO 2 PER CENT.

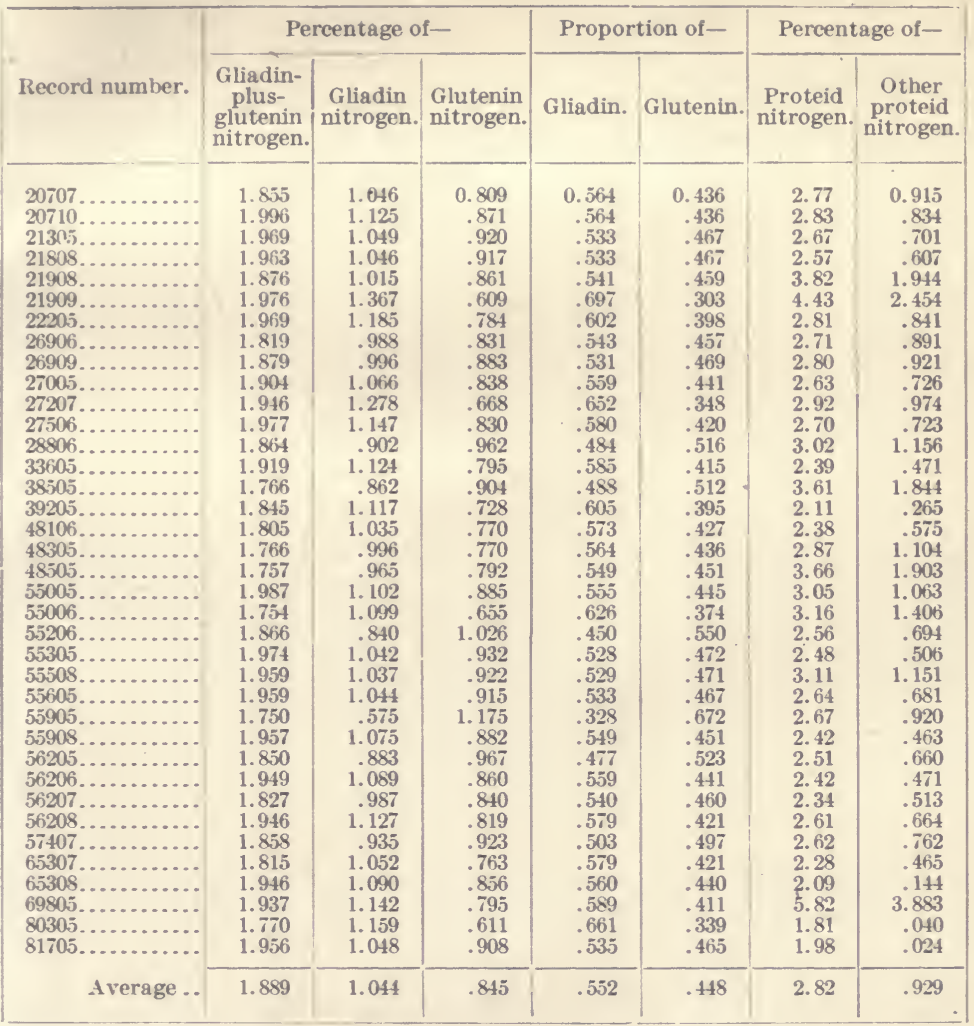

GLIADIN-PLC'S-GLUTENIN NITROGEN, 2 TO 2.25 PER CENT.

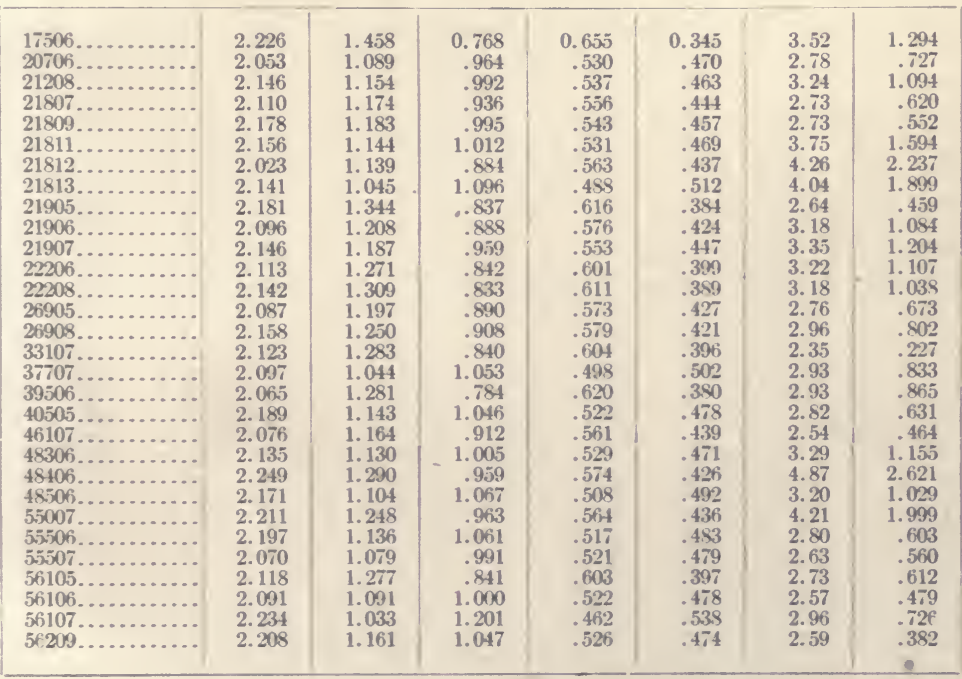


TARLE 23.-Ratio of gliadin to glutenin as the content of their sum increases-Continued.

GLIADIN-PLUS-GLUTENIN NITROGEN, 2 TO 2.25 PER CENT-Continued.

\begin{tabular}{|c|c|c|c|c|c|c|c|}
\hline \multirow[b]{2}{*}{ Record number. } & \multicolumn{3}{|c|}{ Percentage of- } & \multicolumn{2}{|c|}{ Proportion of- } & \multicolumn{2}{|c|}{ Percentage of - } \\
\hline & $\begin{array}{l}\text { Gliadin- } \\
\text { plus- } \\
\text { glutenin } \\
\text { nitrogen. }\end{array}$ & $\begin{array}{l}\text { Gliadin } \\
\text { nitrogen. }\end{array}$ & $\begin{array}{l}\text { Glutenin } \\
\text { nitrogen. }\end{array}$ & Gliadin. & Glutenin. & $\begin{array}{c}\text { Proteid } \\
\text { nitrogen. }\end{array}$ & $\begin{array}{c}\text { Other } \\
\text { proteid } \\
\text { nitrogen. }\end{array}$ \\
\hline $\begin{array}{l}57007 \ldots \ldots \ldots \\
57406 \ldots \ldots \ldots \\
57508 \ldots \ldots \\
58805 \ldots \ldots \ldots \\
63106 \ldots \ldots \ldots \\
66005 \ldots \ldots \ldots \\
74606 \ldots \ldots \ldots \\
76206 \ldots \ldots \ldots \\
81706 \ldots \ldots \ldots \\
\end{array}$ & $\begin{array}{l}2.093 \\
2.134 \\
2.053 \\
2.112 \\
2.199 \\
2.181 \\
2.046 \\
2.029 \\
2.034\end{array}$ & $\begin{array}{l}1.159 \\
1.080 \\
1.124 \\
1.060 \\
1.186 \\
1.142 \\
1.016 \\
1.223 \\
1.701\end{array}$ & $\begin{array}{r}0.934 \\
1.054 \\
.929 \\
1.052 \\
1.013 \\
1.039 \\
1.030 \\
.806 \\
.333\end{array}$ & $\begin{array}{r}0.553 \\
.506 \\
.547 \\
.501 \\
.539 \\
.528 \\
.496 \\
.602 \\
.816\end{array}$ & $\begin{array}{r}0.447 \\
.494 \\
.453 \\
.499 \\
.461 \\
.472 \\
.504 \\
.398 \\
.184\end{array}$ & $\begin{array}{l}2.65 \\
2.75 \\
2.21 \\
2.74 \\
2.79 \\
2.63 \\
2.30 \\
4.45 \\
2.71\end{array}$ & $\begin{array}{r}0.557 \\
.616 \\
.157 \\
.628 \\
.591 \\
.449 \\
.254 \\
2.421 \\
.676\end{array}$ \\
\hline Average.. & 2.130 & 1.187 & .943 & .557 & .443 & 3.05 & .921 \\
\hline
\end{tabular}

GLIADIN-PLUS-GLUTENIN NITROGEN, 2.25 TO 2.50 PER CENT.

\begin{tabular}{|c|c|c|c|c|c|c|c|}
\hline 20709. & 2.313 & 1.307 & 1.006 & 0.565 & 0.435 & 3.05 & 0.737 \\
\hline $20805 \ldots . . . . .$. & 2.259 & 1. 215 & 1.044 & .538 & .462 & 3.32 & 1.061 \\
\hline $20808 \ldots \ldots . . .$. & 2.281 & 1.377 & .904 & .604 & .396 & 3.09 & .809 \\
\hline $27508 \ldots \ldots \ldots$ & 2.324 & 1.247 & 1.077 & .537 & .463 & 2.64 & .316 \\
\hline $28206 \ldots \ldots \ldots$ & 2.424 & 1. 366 & 1.058 & .563 & .437 & 3.07 & .646 \\
\hline $33305 \ldots \ldots \ldots$ & 2.407 & 1. 182 & 1.225 & .491 & .509 & 3.41 & 1.003 \\
\hline $33607 \ldots \ldots$ & 2.446 & 1.391 & 1.055 & .569 & .431 & 3.22 & .774 \\
\hline $34405 \ldots \ldots \ldots$ & 2.443 & 1.230 & 1.213 & .503 & .497 & 4.33 & 1.887 \\
\hline $37305 \ldots \ldots \ldots$ & 2.293 & 1. 208 & 1.085 & .527 & .473 & 2.96 & .667 \\
\hline $57506 \ldots$ & 2.344 & 1.203 & 1.141 & .511 & .489 & 2.80 & .456 \\
\hline $58207 \ldots . . . . .$. & 2.492 & 1.313 & 1. 179 & .526 & .474 & 3.09 & .598 \\
\hline $58705 \ldots . . . \ldots$. & 2.467 & 1.195 & 1.272 & .484 & .516 & 3.01 & .543 \\
\hline Average... & 2.374 & 1.268 & 1.105 & .535 & .465 & 3.16 & .791 \\
\hline
\end{tabular}

GLIADIN-PLUS-GLUTENIN NITROGEN, 2.50 PER CENT AND OVER.

\begin{tabular}{|c|c|c|c|c|c|c|c|}
\hline $\begin{array}{l}40205 \ldots \ldots \ldots \ldots \\
42205 \ldots \ldots \ldots \\
57805 \ldots \ldots \ldots \\
57905 \ldots \ldots \ldots \\
72607 \ldots \ldots \ldots \\
81505 \ldots \ldots \ldots \\
92306 \ldots \ldots \ldots \\
. \ldots \ldots \ldots\end{array}$ & $\begin{array}{l}3.059 \\
2.728 \\
2.684 \\
2.918 \\
2.515 \\
2.652 \\
4.063\end{array}$ & $\begin{array}{l}1.850 \\
1.480 \\
1.303 \\
1.573 \\
1.459 \\
1.066 \\
2.388\end{array}$ & $\begin{array}{l}1.219 \\
1.248 \\
1.381 \\
1.345 \\
1.056 \\
1.586 \\
1.675\end{array}$ & $\begin{array}{r}0.603 \\
.542 \\
.485 \\
.539 \\
.579 \\
.401 \\
.587\end{array}$ & $\begin{array}{r}0.397 \\
.458 \\
.515 \\
.461 \\
.421 \\
.599 \\
.413\end{array}$ & $\begin{array}{l}4.69 \\
3.63 \\
2.87 \\
3.18 \\
5.59 \\
2.94 \\
4.93\end{array}$ & $\begin{array}{r}1.621 \\
.902 \\
.186 \\
.202 \\
3.075 \\
.288 \\
.867\end{array}$ \\
\hline Average... & 2.947 & 1.588 & 1.358 & .534 & .466 & 3.98 & 1.029 \\
\hline
\end{tabular}

TABLE 24.-Summary of analyses, showing the ratio of gliadin to glutenin as the content of their sum increases.

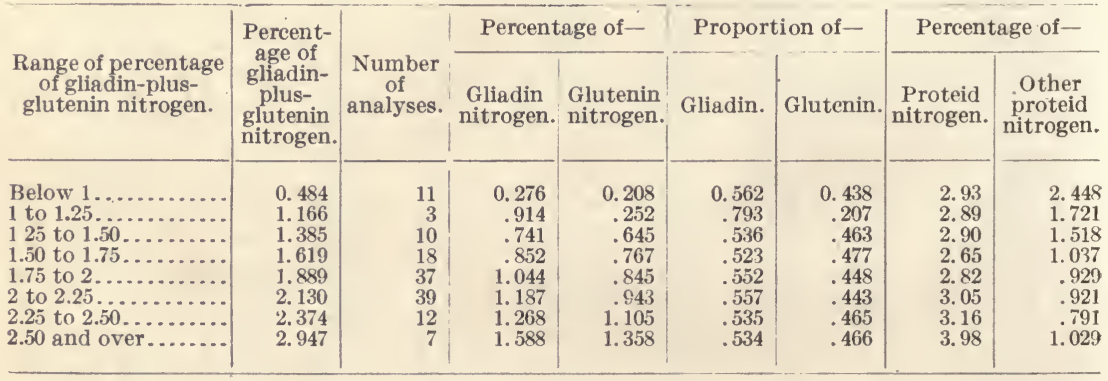

It will be seen from Table 24 that the ratio of gliadin to glutenin remains practically the same as the percentage of their sum increases. It would therefore be safe to assume that an in crease in the gluten 
content of a given variety of wheat raised in the same region would carry with it a corresponding improvement in its value for bread making, although there might be fluctuations from year to year in quality of gluten, as there is in the quantity.

If the quality of gluten is determined by the ratio of gliadin and glutenin of which it is composed, it is likely that there is some certain proportion that is most desirable. Unfortunately, the investigators who have taken up this subject do not by any means agree upon the proper ratio. Should this be ascertained there would be ample opportunity for the selection of individual plants in which the proportion of gliadin and glutenin would approximate the ideal. There would thus be possible a much more rapid improvement in the quality of wheat than can be accomplished by confining selection to an increase in the gluten.

An obstacle to the usefulness of these determinations in the whole wheat appears in the announcement by Nasmith, already cited, that while gliadin occurs in all portions of the endosperm, glutenin does not appear in the aleurone cells. That being the case, it is difficult to believe that any given ratio between these constituents in the whole wheat could be taken as the one most desirable. The ratio in the gluten alone may, however, have an important influence on its quality, and a certain definite proportion of each may produce an ideal gluten.

In the light of the present knowledge on the subject, a mechanical determination of gluten would seem to be most useful, if it can be made with such small quantities of wheat as are obtained from single plants, while determinations of gliadin and glutenin in the gluten would afford a means of judging of its quality.

\section{SOME RESULTS OF BREEDING TO INCREASE THE CONTENT OF PROTEID NITROGEN.}

Selected plants have been grown on a large scale for two years. From these results it is very apparent that a high percentage of nitrogen and the qualities that go with it are transmissible from one generation to another.

In Table 25 are analyses of the plants of the crop of 1902 , grouped according to their proteid nitrogen content into classes of from 1 to 2 per cent, 2 to 2.5 per cent, and increasing by 0.5 per cent up to 4.5 per cent and above. Opposite the plant number of each plant of the crop of 1902 are stated its percentage of proteid nitrogen and weight of proteid nitrogen in kernels. On the same line are the plant numbers for the entire progeny in 1903, and following these are the percentage of proteid nitrogen, weight of proteid nitrogen per average kernel, and average weight of kernel for all of these progeny.

The averages for each group are given in Table 26. 
TABLE 25.-Analyses showing transmission of nitrogen from one generation to another. $a$

1 TO 2 PER CENT PROTEID NITROGEN.

\begin{tabular}{|c|c|c|c|c|c|c|c|}
\hline \multicolumn{4}{|c|}{1902} & \multicolumn{4}{|c|}{1903} \\
\hline $\begin{array}{l}\text { Record num- } \\
\text { ber. }\end{array}$ & $\begin{array}{l}\text { Percent- } \\
\text { age of } \\
\text { proteid } \\
\text { nitrogen } \\
\text { in ker- } \\
\text { nels. }\end{array}$ & $\begin{array}{c}\text { Proteid } \\
\text { nitrogen } \\
\text { in average } \\
\text { kernel } \\
\text { (gram). }\end{array}$ & $\begin{array}{l}\text { Weight of } \\
\text { average } \\
\text { kernel } \\
\text { (gram). }\end{array}$ & $\begin{array}{c}\text { Record num- } \\
\text { ber. }\end{array}$ & $\begin{array}{c}\text { Percent- } \\
\text { age of } \\
\text { proteid } \\
\text { nitrogen } \\
\text { in ker- } \\
\text { nels. }\end{array}$ & $\begin{array}{c}\text { Proteid } \\
\text { nitrogen } \\
\text { in a verage } \\
\text { kernel } \\
\text { (gram). }\end{array}$ & $\begin{array}{l}\text { Weight of } \\
\text { average } \\
\text { kernel } \\
\text { (gram). }\end{array}$ \\
\hline $\begin{array}{l}32201 \ldots \ldots \\
32601 \ldots \ldots \\
63501 \ldots \ldots \\
69501 \ldots \ldots \\
69701 \ldots \ldots \\
73301 \ldots \ldots \\
91901 \ldots \ldots \\
92401 \ldots \ldots \\
92901 \ldots \ldots \\
94101 \ldots \ldots \ldots \\
94201 \ldots \ldots \\
94401 \ldots \ldots \\
94601 \ldots \ldots \\
94901 \ldots \ldots \\
95501 \ldots \ldots \\
\end{array}$ & $\begin{array}{l}1.51 \\
1.99 \\
1.98 \\
1.94 \\
1.97 \\
1.12 \\
1.83 \\
1.33 \\
1.67 \\
1.38 \\
1.63 \\
1.73 \\
1.89 \\
1.99 \\
1.92\end{array}$ & $\begin{array}{l}\cdots \\
\cdots \\
\cdots \\
\cdots \\
\cdots \\
\cdots \\
\cdots \\
\cdots \\
\cdots \\
\cdots \\
\cdots\end{array}$ & 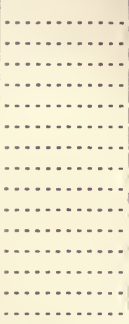 & 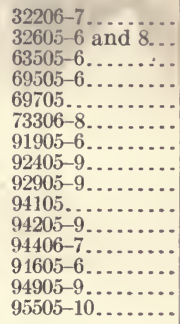 & $\begin{array}{l}2.64 \\
2.62 \\
2.17 \\
2.39 \\
2.50 \\
2.586 \\
3.09 \\
2.628 \\
2.814 \\
2.67 \\
2.576 \\
2.27 \\
1.87 \\
2.85 \\
2.498\end{array}$ & $\begin{array}{r}0.0010055 \\
.0015963 \\
.0007499 \\
.0009348 \\
.0003874 \\
.0016918 \\
.0010830 \\
.0024129 \\
.0024540 \\
.0006790 \\
.0022132 \\
.0008092 \\
.0016125 \\
.0025361 \\
.0026506\end{array}$ & $\begin{array}{l}0.03874 \\
.07560 \\
.03502 \\
.03894 \\
.01550 \\
.06582 \\
.03513 \\
.09109 \\
.08814 \\
.02543 \\
.08738 \\
.03538 \\
.08851 \\
.08899 \\
.10605\end{array}$ \\
\hline Average. . & 1.658 & & & Average . & 2.587 & .0004960 & .019907 \\
\hline
\end{tabular}

$a$ In this table the average percentage of proteid nitrogen for all plants raised in 1903, resulting from plants of 1 to 2 per cent, 2 to 2.5 per cent, etc., in 1902 is determined by adding together analyses of all plants in that group and dividing by the total number, irrespective of families.

2 TO 2.5 PER CENT PROTEID NITROGEN.

\begin{tabular}{|c|c|c|c|c|c|c|c|}
\hline $\begin{array}{l}17401 \ldots \ldots \ldots \ldots \\
34201 \ldots \ldots \ldots \ldots \\
57301 \ldots \ldots \ldots\end{array}$ & $\begin{array}{l}2.45 \\
2.28 \\
2.33\end{array}$ & 0.000601 & 0.02585 & $\begin{array}{l}1740[5-6][8-10] \\
34205-8 \ldots \ldots \ldots \\
57305-8 \ldots \ldots\end{array}$ & $\begin{array}{l}2.646 \\
2.857 \\
2.54\end{array}$ & $\begin{array}{r}0.0025803 \\
.0023077 \\
.0018351\end{array}$ & $\begin{array}{r}0.09807 \\
.08075 \\
.07010\end{array}$ \\
\hline Average. . & 2.353 & .000601 & .02585 & Average . & 2. 68 & .00051716 & .019146 \\
\hline
\end{tabular}

2.5 TO 3 PER CENT PROTEID NITROGEN.

\begin{tabular}{|c|c|c|c|c|c|c|c|}
\hline $\begin{array}{l}21701 \ldots \ldots \ldots \\
33401 \ldots \ldots\end{array}$ & $\begin{array}{l}2.50 \\
2.73\end{array}$ & & & $\begin{array}{l}21705-11 \ldots \ldots \ldots \\
33405-8 . \ldots \ldots\end{array}$ & $\begin{array}{l}2.78 \\
1.977\end{array}$ & $\begin{array}{r}0.0042343 \\
.0014277\end{array}$ & $\begin{array}{r}0.15101 \\
.07274\end{array}$ \\
\hline Average. . & 2.615 & & & Average & 2.487 & .0005147 & .02032 \\
\hline
\end{tabular}

3 TO 3.5 PER CENT PROTEID NITROGEN.

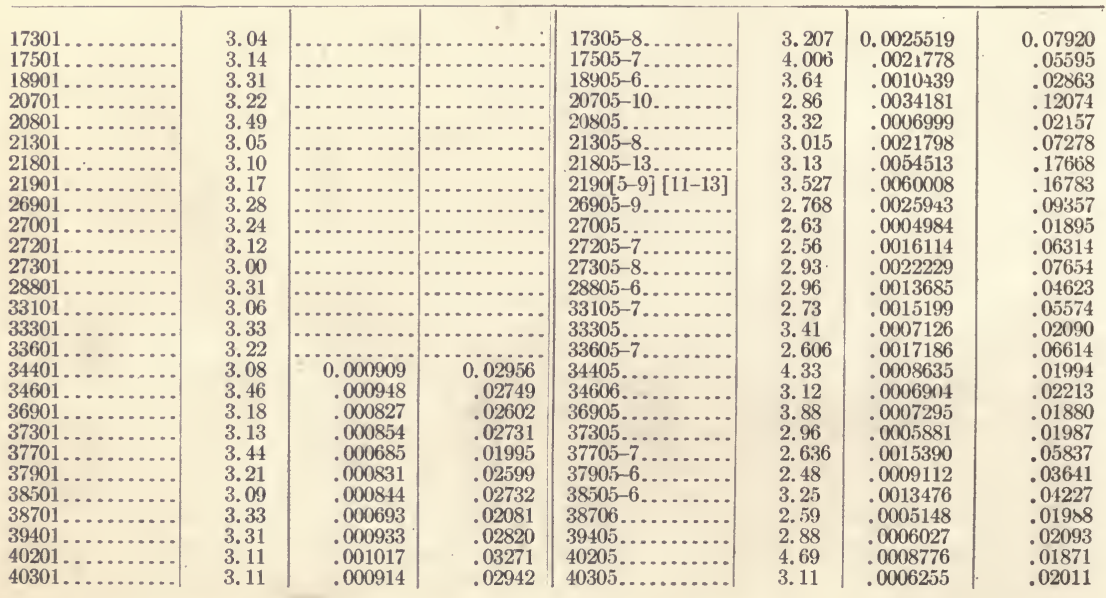


TABLE 25.-Analyses showing transmission of nitrogen from one generation to anotherContinued.

3 TO 3.5 PER CENT PROTEID NITROGEN-Continued.

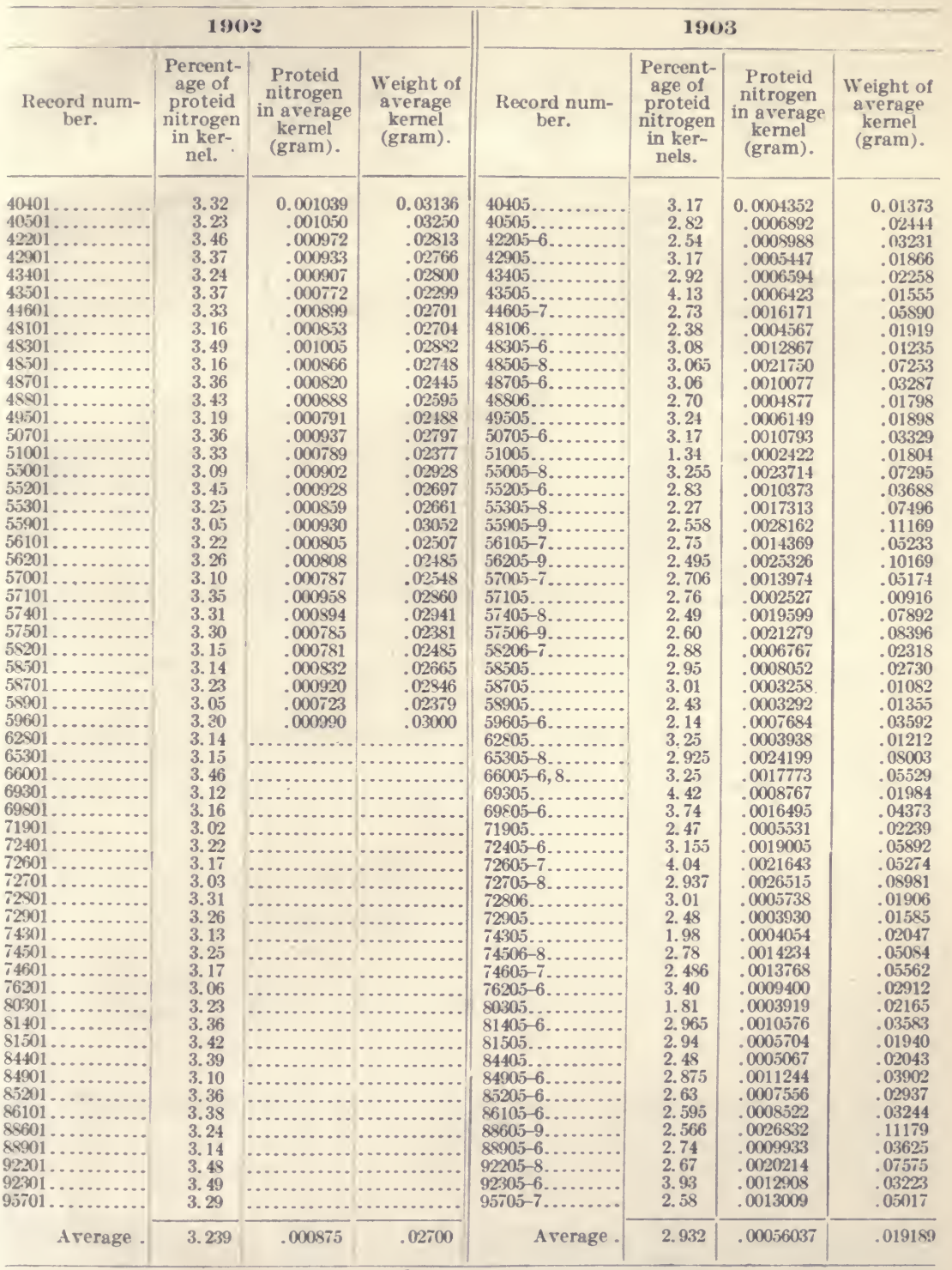

3.5 TO 4 PER CENT PROTEID NITROGEN.

\begin{tabular}{|c|c|c|c|c|c|c|c|}
\hline $18401 \ldots$ & 3.55 & & & 18805 & 2.02 & 0.0003164 & 0.01567 \\
\hline $22201 \ldots \ldots \ldots$ & 3.65 & & & $22205-11 \ldots \ldots$ & 3. 165 & .0037042 & .11711 \\
\hline $26101 \ldots \ldots$ & 3. 76 & & $\ldots \ldots$ & $26105-7 \ldots$ & 3.19 & .0015273 & .05113 \\
\hline $27501 \ldots \ldots$ & 3.58 & & $\ldots \ldots$ & $27505-9 \ldots \ldots$ & 2.688 & .0028791 & .10761 \\
\hline
\end{tabular}


TABLE 25.-Analyses showing transmission of nitrogen from one generation to anotherContinued.

3.5 TO 4 PER CENT PROTEID NITROGEN-Continued.

\begin{tabular}{|c|c|c|c|c|c|c|c|}
\hline \multicolumn{4}{|c|}{1902} & \multicolumn{4}{|c|}{1903} \\
\hline $\begin{array}{l}\text { Record num- } \\
\text { ber. }\end{array}$ & $\begin{array}{l}\text { Percent- } \\
\text { age of } \\
\text { proteid } \\
\text { nitrogen } \\
\text { in ker- } \\
\text { nels. }\end{array}$ & $\begin{array}{c}\text { Proteid } \\
\text { nitrogen } \\
\text { in average } \\
\text { kernel } \\
(\mathrm{gram}) .\end{array}$ & $\begin{array}{l}\text { Weight of } \\
\text { average } \\
\text { kernel } \\
\text { (gram). }\end{array}$ & $\begin{array}{l}\text { Record num- } \\
\text { ber. }\end{array}$ & $\begin{array}{l}\text { Percent- } \\
\text { age of } \\
\text { proteid } \\
\text { nitrogen } \\
\text { in ker- } \\
\text { nels. }\end{array}$ & $\begin{array}{c}\text { Proteid } \\
\text { nitrogen } \\
\text { in average } \\
\text { kernel } \\
\text { (gram). }\end{array}$ & $\begin{array}{l}\text { Weight of } \\
\text { average } \\
\text { kernel } \\
\text { (gram). }\end{array}$ \\
\hline 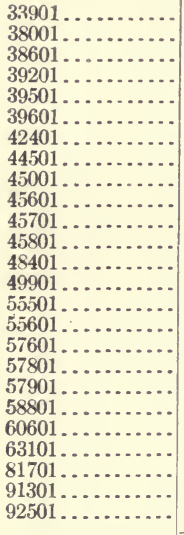 & $\begin{array}{l}3.59 \\
3.82 \\
3.79 \\
3.98 \\
3.65 \\
3.55 \\
3.63 \\
3.57 \\
3.79 \\
3.87 \\
3.55 \\
3.87 \\
3.53 \\
3.61 \\
3.55 \\
3.79 \\
3.76 \\
3.80 \\
3.64 \\
3.80 \\
3.53 \\
3.91 \\
3.78 \\
3.57 \\
3.56 \\
\end{array}$ & $\begin{array}{r}.000806 \\
.001046 \\
.001039 \\
.001048 \\
.000927 \\
.001327 \\
.000796 \\
.001020 \\
.001238 \\
.000865 \\
.001146 \\
.000993 \\
.001043 \\
.001020 \\
.001050 \\
.001030 \\
.000891 \\
.000852 \\
.000904 \\
.000759 \\
\ldots . . . . . \\
\ldots \ldots . . .\end{array}$ & \begin{tabular}{|c|}
$\ldots .02110$ \\
.02765 \\
.02616 \\
.02877 \\
.02619 \\
.02338 \\
.02531 \\
.02690 \\
.03205 \\
.02435 \\
.02963 \\
.02822 \\
.02898 \\
.02866 \\
.02775 \\
.02750 \\
.02353 \\
.02348 \\
.02384 \\
.02155 \\
$\ldots \ldots . .$.
\end{tabular} & 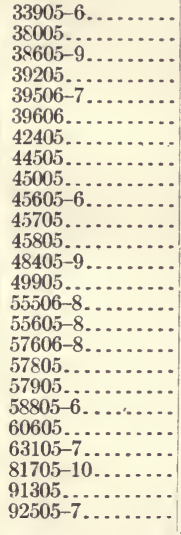 & $\begin{array}{l}2.21 \\
2.84 \\
3.718 \\
2.11 \\
2.975 \\
2.37 \\
3.07 \\
2.94 \\
3.58 \\
2.365 \\
4.18 \\
1.84 \\
2.90 \\
3.62 \\
2.846 \\
2.555 \\
2.37 \\
2.87 \\
3.18 \\
2.31 \\
1.87 \\
2.82 \\
2.27 \\
3.21 \\
3.32\end{array}$ & $\begin{array}{l}0.0008932 \\
.0005135 \\
.0036318 \\
.0004407 \\
.0013536 \\
.0003177 \\
.0006927 \\
.0005187 \\
.0004927 \\
.0006777 \\
.0007155 \\
.0002700 \\
.0020794 \\
.0010640 \\
.0016285 \\
.0022356 \\
.0015451 \\
.0005207 \\
.0003556 \\
.0009317 \\
.0003180 \\
.0016570 \\
.0031019 \\
.0007197 \\
.0017483 \\
\end{array}$ & $\begin{array}{r}0.04115 \\
.01808 \\
.09917 \\
.02089 \\
.04568 \\
.01341 \\
.02251 \\
.01764 \\
.01376 \\
.02995 \\
.01712 \\
.01234 \\
.07511 \\
.02939 \\
.05743 \\
.05822 \\
.06535 \\
.01814 \\
.01118 \\
.04048 \\
.01701 \\
.05951 \\
.13635 \\
.02242 \\
.05312 \\
\end{array}$ \\
\hline
\end{tabular}

4 TO 4.5 PER CENT PROTEID NITROGEN.

\begin{tabular}{|c|c|c|c|c|c|c|c|}
\hline $\begin{array}{l}26801 \ldots \ldots \ldots \ldots \\
28201 \ldots \ldots \ldots \ldots \\
46101 \ldots \ldots\end{array}$ & $\begin{array}{l}4.07 \\
4.30 \\
4.00\end{array}$ & $\begin{array}{r}\ldots \ldots \ldots \ldots \\
0.000988\end{array}$ & ......... & $\begin{array}{l}26805-8 \ldots \ldots \ldots \\
28206 . \ldots \ldots \ldots \\
46105-7 \ldots \ldots \ldots\end{array}$ & $\begin{array}{l}2.825 \\
3.07 \\
2.69\end{array}$ & $\begin{array}{r}0.0023073 \\
.0006126 \\
.0014772\end{array}$ & $\begin{array}{r}0.08179 \\
.01996 \\
.05495\end{array}$ \\
\hline Average. & 4. 123 & .000988 & .02472 & Average. & 2.806 & .0005496 & .019588 \\
\hline
\end{tabular}

MORE THAN 4.5 PER CENT PROTEID NITROGEN.

\begin{tabular}{|c|c|c|c|c|c|c|c|}
\hline $50901 \ldots \ldots \ldots$. & 4.95 & 0.001074 & 0.02171 & \multirow{2}{*}{$\begin{array}{r}50905-6 . . . . . . \\
\text {-Average }\end{array}$} & 3. 435 & 0.0008992 & 0.02001 \\
\hline Average. & $\ldots \ldots \ldots$ & ............ & $\ldots \ldots \ldots$ & & 3.435 & .0004496 & .010005 \\
\hline
\end{tabular}

TABLE 26.-Summary of analyses, showing transmission of nitrogen from one generation to another.

\begin{tabular}{|c|c|c|c|c|c|c|c|c|}
\hline \multirow[b]{2}{*}{$\begin{array}{l}\text { Range of percentage of } \\
\text { proteid nitrogen. }\end{array}$} & \multicolumn{4}{|c|}{1902} & \multicolumn{4}{|c|}{1903} \\
\hline & $\begin{array}{l}\text { Percent- } \\
\text { age of } \\
\text { proteid } \\
\text { nitrogen } \\
\text { in ker- } \\
\text { nels. }\end{array}$ & $\begin{array}{c}\text { Num- } \\
\text { ber of } \\
\text { analy- } \\
\text { ses. }\end{array}$ & $\begin{array}{c}\text { Proteid } \\
\text { nitrogen } \\
\text { in average } \\
\text { kernel } \\
\text { (gram). }\end{array}$ & $\begin{array}{l}\text { Weight } \\
\text { of aver- } \\
\text { age ker- } \\
\text { nel } \\
\text { (gram). }\end{array}$ & $\begin{array}{l}\text { Percent- } \\
\text { age of } \\
\text { proteid } \\
\text { nitrogen } \\
\text { in ker- } \\
\text { nels. }\end{array}$ & $\begin{array}{l}\text { Num- } \\
\text { ber of } \\
\text { analy- } \\
\text { ses. }\end{array}$ & $\begin{array}{c}\text { Proteid } \\
\text { nitrogen } \\
\text { in average } \\
\text { kernel } \\
\text { (gram). }\end{array}$ & $\begin{array}{c}\text { Weight } \\
\text { of aver- } \\
\text { age ker- } \\
\text { nel } \\
\text { (gram). }\end{array}$ \\
\hline 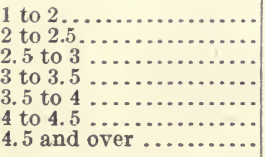 & $\begin{array}{l}1.66 \\
2.35 \\
2.61 \\
3.24 \\
3.68 \\
4.12 \\
4.95\end{array}$ & $\begin{array}{r}15 \\
3 \\
2 \\
84 \\
31 \\
3 \\
1\end{array}$ & $\begin{array}{c}0.000601 \\
0.0007 \overline{7} \\
.000990 \\
.000988 \\
.001074\end{array}$ & $\begin{array}{c}0.02585 \\
.02700 \\
.02650 \\
.02472 \\
.02171\end{array}$ & $\begin{array}{l}2.59 \\
2.68 \\
2.49 \\
2.93 \\
2.91 \\
2.81 \\
3.43\end{array}$ & $\begin{array}{r}46 \\
13 \\
11 \\
199 \\
79 \\
8 \\
2\end{array}$ & $\begin{array}{r}0.0004960 \\
.0005172 \\
.0005147 \\
.0005604 \\
.0005508 \\
.0005496 \\
.0004496\end{array}$ & $\begin{array}{r}0.01991 \\
.01915 \\
.02032 \\
.01919 \\
.01920 \\
.01959 \\
.01000\end{array}$ \\
\hline
\end{tabular}


In Table 26 the averages for each group are stated. This table is designed to show whether there has been a tendency for plants of a certain class to reproduce the qualities pertaining to that class, or whether these are lost in the offspring.

It is unfortunate that there are not a greater number of analyses of plants of medium and of low nitrogen content. The plants selected for reproduction in 1903 were largely those of high nitrogen content, and, consequently, comparatively few analyses of the low nitrogen and medium nitrogen plants of 1903 are at hand.

Table 25 shows that in the main there is a tendency for each class of plants to reproduce in the same relation to the other classes, but that there is less difference between the extreme classes in the offspring than in the parent plants. In other words, while all plants tend to reproduce their own qualities, those plants varying widely from the average produce, in general, offspring varying from the average less widely than did the parents. Although this is a rule, its application to the individual is not universal. Certain plants may be found whose tendency to variation extends through both generations. There is also wide variation between certain plants of the same parent. For instance, the plants numbered from 21205 to 21212 , all of which come from the same parent, vary from 2.16 to 5.23 per cent in proteid nitrogen content, while plants 69805 and 69806 vary from 5.82 to 1.66 per cent in this constituent. ${ }^{a}$

It would seem, therefore, entirely reasonable to believe that a very considerable increase in the proteid nitrogen content of wheat may be effected by careful and continuous reproduction from plants of high proteid nitrogen content.

Table 27 contains the analyses of plants raised in 1902 and their progeny raised in 1903, arranged according to the number of grams of proteid nitrogen contained in the average kernel of the former.

TABLE 27.-Analyses showing transmission of proteid nitrogen in average kernel.

\begin{tabular}{|c|c|c|c|c|c|c|c|c|}
\hline \multirow[b]{2}{*}{$\begin{array}{l}\text { Range of proteid nitrogen } \\
\text { in a v e } \mathrm{r} \text { a g e kernel } \\
\text { (gram). }\end{array}$} & \multicolumn{4}{|c|}{$190^{\circ}$} & \multicolumn{4}{|c|}{1903} \\
\hline & $\begin{array}{c}\text { Proteid } \\
\text { nitrogen } \\
\text { in arer- } \\
\text { age ker- } \\
\text { nel } \\
(\text { gram). }\end{array}$ & $\begin{array}{l}\text { Num- } \\
\text { ber of } \\
\text { anal- } \\
\text { yses. }\end{array}$ & $\begin{array}{c}\text { Percent- } \\
\text { age of } \\
\text { proteid } \\
\text { nitrogen } \\
\text { in ker- } \\
\text { nels. }\end{array}$ & $\begin{array}{l}\text { Weight } \\
\text { of aver- } \\
\text { age ker- } \\
\text { nel } \\
\text { (gram). }\end{array}$ & $\begin{array}{c}\text { Proteid } \\
\text { nitrogen } \\
\text { in aver- } \\
\text { age ker- } \\
\text { nel } \\
\text { (gram). }\end{array}$ & $\begin{array}{l}\text { Num- } \\
\text { ber of } \\
\text { anal- } \\
\text { yses. }\end{array}$ & $\begin{array}{c}\text { Percent- } \\
\text { age of } \\
\text { proteid } \\
\text { nitrogen } \\
\text { in ker- } \\
\text { nels. }\end{array}$ & $\begin{array}{l}\text { Weight } \\
\text { of aver- } \\
\text { age ker- } \\
\text { nel } \\
\text { (gram). }\end{array}$ \\
\hline $\begin{array}{l}0.000600 \text { to } 0.000700 \ldots \\
0.000700 \text { to } 0.000800 \ldots \\
0.000500 \text { to } 0.000900 \ldots \\
0.000900 \text { to } 0.001000 \ldots \\
0.001000 \text { and over } \ldots\end{array}$ & $\begin{array}{r}0.000659 \\
.000776 \\
.000850 \\
.000938 \\
.001077\end{array}$ & $\begin{array}{r}3 \\
9 \\
18 \\
18 \\
15\end{array}$ & $\begin{array}{l}3.03 \\
\text { 3. } 29 \\
3.33 \\
3.37 \\
3.71\end{array}$ & $\begin{array}{r}0.02220 \\
.02405 \\
.02576 \\
.02796 \\
.02880\end{array}$ & $\begin{array}{r}0.000496 \\
.000444 \\
.000544 \\
.000514 \\
.000593\end{array}$ & $\begin{array}{r}8 \\
15 \\
38 \\
35 \\
28\end{array}$ & $\begin{array}{l}2.59 \\
2.68 \\
2.91 \\
2.89 \\
3.06\end{array}$ & $\begin{array}{l}0.01895 \\
.01673 \\
.01875 \\
.01784 \\
.01905\end{array}$ \\
\hline
\end{tabular}

( Table 25 represents the properties of each plant grown in 1903 arranged according to immediate families. For instance, plants numbered $17305-17308$ are all the off spring of the same plant grown in 1902 . The parent bears the number 17301. This is the system of records devised by Prof. IV. M. Hays, formerly of the University of Minnesota. 
TABLE 28.-Analyses showing transmission of kernel weight.

\begin{tabular}{|c|c|c|c|c|c|c|c|c|}
\hline \multirow[b]{2}{*}{$\begin{array}{l}\text { Range of weight of aver- } \\
\text { age kernel (gram). }\end{array}$} & \multicolumn{4}{|c|}{1902} & \multicolumn{4}{|c|}{1903} \\
\hline & $\begin{array}{c}\text { Weight } \\
\text { of aver- } \\
\text { age ker- } \\
\text { nel } \\
\text { (gram). }\end{array}$ & $\begin{array}{l}\text { Num- } \\
\text { ber of } \\
\text { anal- } \\
\text { yses. }\end{array}$ & $\begin{array}{l}\text { Percent- } \\
\text { age of } \\
\text { proteid } \\
\text { nitrogen } \\
\text { in ker- } \\
\text { nels. }\end{array}$ & $\begin{array}{c}\text { Proteid } \\
\text { nitrogen } \\
\text { in aver- } \\
\text { age ker- } \\
\text { nel } \\
\text { (gram). }\end{array}$ & $\begin{array}{l}\text { Weight } \\
\text { of aver- } \\
\text { age ker- } \\
\text { nel } \\
\text { (gram). }\end{array}$ & $\begin{array}{l}\text { Num- } \\
\text { ber of } \\
\text { anal- } \\
\text { yses. }\end{array}$ & $\begin{array}{l}\text { Percent- } \\
\text { age of } \\
\text { proteid } \\
\text { nitrogen } \\
\text { in ker- } \\
\text { nels. }\end{array}$ & $\begin{array}{l}\text { Proteid } \\
\text { nitrogen } \\
\text { in aver- } \\
\text { age ker- } \\
\text { nel } \\
\text { (gram). }\end{array}$ \\
\hline 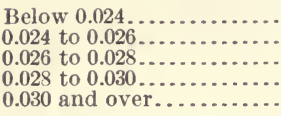 & $\begin{array}{l}0.02253 \\
.02515 \\
.02709 \\
.02878 \\
.03152\end{array}$ & $\begin{array}{r}12 \\
12 \\
18 \\
16 \\
6\end{array}$ & $\begin{array}{l}\text { 3. } 61 \\
3.28 \\
3.43 \\
3.41 \\
3.31\end{array}$ & $\begin{array}{l}0.000811 \\
.000813 \\
.000927 \\
.000993 \\
.001044\end{array}$ & $\begin{array}{r}0.01684 \\
.01740 \\
.01947 \\
.01875 \\
.01869\end{array}$ & $\begin{array}{l}19 \\
28 \\
38 \\
31 \\
12\end{array}$ & $\begin{array}{l}2.69 \\
2.88 \\
2.91 \\
2.98 \\
2.96\end{array}$ & $\begin{array}{r}0.000450 \\
.000503 \\
.000562 \\
.000573 \\
.000548\end{array}$ \\
\hline
\end{tabular}

Table 28 shows the analyses of plants raised in 1902 and their progeny raised in 1903, arranged according to weight of average kernel. There is more variation in this table than in the preceding one, but the tendency toward transmission of proteid nitrogen in the average kernel may be noted. The averages for 1902 are much higher than for 1903 , owing partly to the higher percentage and partly to greater kernel weight.

The weight of the average kernel shows some tendency toward transmission, although there are some variations. It will be noticed that the kernels average much heavier in 1902 than in 1903, and that in spite of this the percentage of proteid nitrogen is higher in 1902 . The relation of light kernel and high percentage of nitrogen does not therefore appear to hold as between crops of different years.

All of the qualities of which determinations have been made in both years appear to be transmitted. It may be safely assumed that certain plants will have greater power to transmit these qualities than will the average plant. Such plants will assert themselves in the course of three or four generations. From these plants individuals may be selected that have a combination of the desired qualities.

\section{YIELD OF GRAIN AS AFFECTED BY SUSCEPTIBILITY TO COLD.}

As has already been stated, a large number of plants on the breeding plots were killed during the winter of 1902-3. This afforded an opportunity to ascertain the effect of the severe weather upon the surviving plants. The question arose whether the surviving plants of a family of which a large percentage of members were killed yielded less per plant than the plants of a family of which but a small percentage had succumbed. As each spike of the crop of 1902 was represented by a number of plants, and as records of each plant were available, there were very extensive data at hand from which to secure information on the subject.

In Table 29 the surviving plants of each immediate family, or, in other words, the surviving plants descended from the same plant of the previous year, are classified according to the percentage of plants that survived the winter. Thus all plants of which only from 10 to 20 
per cent survived are grouped together. In the next class are all plants of which from 20 to 30 per cent survived. The other classes increase by 10 per cent surviving plants until 70 per cent is reached. All plants of which more than 70 per cent survived form the last class.

Table 30 gives å summary of Table 29, the averages for each class being shown. From this table it will be seen that with an increase in the proportion of surviving plants there is an increase in the weight of grain per plant and in the number of kernels per plant. It is therefore to be concluded that decrease in yield from winterkilling is due not only to the loss of plants that are destroyed, but also to a decreased yield from most of the surviving plants.

Table 30 also shows that the weight of the average kernel is not affected by the freezing of a large proportion of the family, the decreased yield being due, it may be assumed, to the decreased number of kernels, owing to a decreased ability to tiller.

With an increase in the proportion of surviving plants there is, perhaps, a slight decrease in the percentage of proteid nitrogen in the kernels and in the number of grams of proteid nitrogen in the average kernel, although this is so slight and so irregular that it would not be safe to draw any conclusions from it. The total production of proteid nitrogen per plant naturally increases.

TABLE 29.-Yields of plants, arranged according to percentage killed in each family.

10 TO 20 PER CENT.

\begin{tabular}{|c|c|c|c|c|c|c|c|}
\hline $\begin{array}{l}\text { Record number } \\
\text { for } 1902 .\end{array}$ & $\begin{array}{l}\text { Percent- } \\
\text { age of } \\
\text { plants } \\
\text { in } 1903 \\
\text { surviv- } \\
\text { ing from } \\
1902 .\end{array}$ & $\begin{array}{l}\text { Weight } \\
\text { of ker- } \\
\text { nels on } \\
\text { plant } \\
\text { (gram). }\end{array}$ & $\begin{array}{c}\text { Num- } \\
\text { ber of } \\
\text { kernels. }\end{array}$ & $\begin{array}{l}\text { Weight } \\
\text { of aver- } \\
\text { age } \\
\text { kernel } \\
\text { (gram). }\end{array}$ & $\begin{array}{l}\text { Percent- } \\
\text { age of } \\
\text { proteid } \\
\text { nitrogen } \\
\text { in ker- } \\
\text { nels. }\end{array}$ & $\begin{array}{l}\text { Proteid } \\
\text { nitrogen } \\
\text { in ker- } \\
\text { nels } \\
\text { (gram). }\end{array}$ & $\begin{array}{c}\text { Proteid } \\
\text { nitrogen } \\
\text { in average } \\
\text { kernel } \\
\text { (gram). }\end{array}$ \\
\hline $18801 \ldots \ldots \ldots \ldots$ & 11.1 & 2. 1462 & 137 & 0.01567 & 2.02 & 0.04335 & 0.0003164 \\
\hline $20801 \ldots \ldots \ldots$ & 10.0 & 14.6942 & 697 & .02157 & 3.32 & .48781 & .0006999 \\
\hline $25201 \ldots \ldots$ & 18.2 & 7.7295 & 363 & .02173 & 2.73 & .20732 & .0005947 \\
\hline $33301 \ldots . . .$. & 16.7 & 2.9905 & 156 & .01858 & 2.73 & .07566 & .0005066 \\
\hline $37301 \ldots \ldots$ & $16 . .7$ & 6. 1394 & 309 & .01987 & 2.96 & .18173 & .0000881 \\
\hline $38001 \ldots \ldots$ & 14.3 & 2.5134 & 139 & .01808 & 2.81 & .07138 & .0005135 \\
\hline $39201 \ldots \ldots$ & 16.7 & 21.5399 & 1,031 & .02089 & 2.11 & .45435 & .0004407 \\
\hline 39401 . & 16.7 & 9.3541 & 447 & .02093 & 2.88 & .21399 & .0006027 \\
\hline $40201 \ldots$ & 14.3 & 3.6302 & 194 & .01871 & 4. 69 & .17026 & .0008776 \\
\hline $40401 \ldots$ & 16.7 & .6316 & 46 & .01373 & 3.17 & .02002 & .0004352 \\
\hline $42901 \ldots$ & 16.7 & 1.2499 & 67 & .01866 & 3.17 & .03650 & .0005447 \\
\hline 43401 & 16.7 & 2.8000 & 124 & .02258 & 2.92 & .08176 & .0006594 \\
\hline 44501 . & 16.7 & 5.9990 & 340 & .01764 & 2.94 & .17637 & .0005187 \\
\hline $45001 \ldots$ & 16.7 & 3. 2340 & 235 & .01376 & 3.58 & .11575 & .0004927 \\
\hline 4570 & 14.3 & 32 & 44 & .01712 & 4. 18 & .03148 & .0007155 \\
\hline 4580 & 16.7 & 1. 5298 & 124 & .01234 & 1.84 & .02815 & .0002700 \\
\hline $49501 \ldots \ldots \ldots$ & 14.3 & 1.2716 & 67 & .01898 & 3.24 & .04120 & .0006149 \\
\hline $49901 \ldots \ldots \ldots$ & 14.3 & .6760 & 23 & .02939 & 3.62 & .02436 & .0010640 \\
\hline 51001 . & 16.7 & 15.5835 & 862 & .018 & 1.34 & .20881 & .0002422 \\
\hline 57101. & 16.7 & 3. 7263 & 407 & .00916 & 2.76 & .10285 & .0002527 \\
\hline 58501 .. & 16.7 & 7.4516 & 273 & .02730 & 2.95 & .21982 & .0008052 \\
\hline $58701 \ldots$ & 16.7 & 2.5436 & 235 & .01082 & 3.01 & .07656 & .0003258 \\
\hline $58901 \ldots \ldots \ldots$. & 16.7 & 2. 3031 & 170 & .01355 & 2.43 & .05596 & .0003292 \\
\hline $60601 \ldots$ & 16.7 & .5952 & 35 & .017 & 1.87 & .01113 & .0003180 \\
\hline 62 & 16.7 & 1.3451 & 111 & .01212 & 3.25 & .04272 & .0003938 \\
\hline 69 & 16.7 & 2.0430 & 103 & .01984 & 4.42 & .09030 & .0008767 \\
\hline $1 .$. & 16.7 & 4. 4222 & 216 & .02047 & 1.98 & .08756 & .0004054 \\
\hline 84 & 16.7 & 8.7448 & 428 & .020 & 2.48 & .21687 & .0005067 \\
\hline 91 & $14 . ?$ & 3.0940 & 138 & .022 & 3.21 & .09932 & .0007197 \\
\hline 9410 & 14. & .5595 & 22 & .02543 & 2.67 & .01494 & .0006790 \\
\hline Averag & 13 & 4. 7098 & 9514 & .01856 & 2.91 & .12294 & .000513666 \\
\hline
\end{tabular}


TABLE 29.- - Yields of plants, arranged according to percentage killed in each family-Cont'd.

20 TO 30 PER CENT.

\begin{tabular}{|c|c|c|c|c|c|c|c|}
\hline $\begin{array}{l}\text { Record number } \\
\text { for } 1902 .\end{array}$ & $\begin{array}{c}\text { Percent- } \\
\text { age of } \\
\text { plants } \\
\text { in } 1903 \\
\text { surviv- } \\
\text { ing from } \\
1902 .\end{array}$ & $\begin{array}{l}\text { Weight } \\
\text { of ker- } \\
\text { nels on } \\
\text { plant } \\
\text { (gram). }\end{array}$ & $\begin{array}{c}\text { Num- } \\
\text { ber of } \\
\text { kernels. }\end{array}$ & $\begin{array}{c}\text { Weight } \\
\text { of aver- } \\
\text { age } \\
\text { kernel } \\
\text { (gram). }\end{array}$ & $\begin{array}{c}\text { Percent- } \\
\text { age of } \\
\text { proteid } \\
\text { nitrogen } \\
\text { in ker- } \\
\text { nels. }\end{array}$ & $\begin{array}{c}\text { Proteid } \\
\text { nitrogen } \\
\text { in ker- } \\
\text { nels } \\
\text { (gram). }\end{array}$ & $\begin{array}{c}\text { Proteid } \\
\text { nitrogen } \\
\text { in average } \\
\text { kernel } \\
\text { (gram). }\end{array}$ \\
\hline 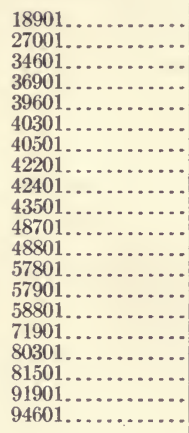 & $\begin{array}{l}20.0 \\
20.0 \\
28.6 \\
20.0 \\
28.6 \\
25.0 \\
20.0 \\
25.0 \\
20.0 \\
25.0 \\
28.6 \\
25.0 \\
20.0 \\
25.0 \\
28.6 \\
20.0 \\
20.0 \\
20.0 \\
22.2 \\
28.6\end{array}$ & $\begin{array}{r}1.2046 \\
16.4120 \\
6.1962 \\
5.0200 \\
4.6383 \\
3.6003 \\
4.1546 \\
1.0827 \\
1.4892 \\
1.4464 \\
5.2800 \\
9.8346 \\
4.8988 \\
2.4731 \\
12.5470 \\
28.2136 \\
15.7835 \\
2.8327 \\
3.4961 \\
6.2877\end{array}$ & $\begin{array}{r}84 \\
866 \\
280 \\
267 \\
346 \\
179 \\
170 \\
59 \\
66 \\
93 \\
321 \\
547 \\
270 \\
221 \\
626 \\
1,260 \\
729 \\
146 \\
199 \\
106\end{array}$ & $\begin{array}{l}0.01431 \\
.01895 \\
.02213 \\
.01880 \\
.01341 \\
.02011 \\
.02444 \\
.01615 \\
.02251 \\
.01555 \\
.01643 \\
.01798 \\
.01814 \\
.01118 \\
.02024 \\
.02239 \\
.02165 \\
.01940 \\
.01756 \\
.04425\end{array}$ & $\begin{array}{l}3.64 \\
2.63 \\
3.12 \\
3.88 \\
2.37 \\
3.11 \\
2.82 \\
2.54 \\
3.07 \\
4.13 \\
3.06 \\
2.70 \\
2.87 \\
3.18 \\
2.31 \\
2.47 \\
1.81 \\
2.94 \\
3.09 \\
1.87\end{array}$ & $\begin{array}{l}0.04437 \\
.43164 \\
.19332 \\
.19478 \\
.10967 \\
.11197 \\
.11716 \\
.03587 \\
.04572 \\
.05974 \\
.16124 \\
.26553 \\
.14060 \\
.07859 \\
.33541 \\
.69688 \\
.28569 \\
.08328 \\
.10771 \\
.11373\end{array}$ & $\begin{array}{r}0.0005219 \\
.0004981 \\
.0006904 \\
.0007295 \\
.0003177 \\
.0006255 \\
.0006892 \\
.0004194 \\
.0006927 \\
.0006423 \\
.0005038 \\
.0004877 \\
.0005207 \\
.0003556 \\
.0004658 \\
.0005531 \\
.0003919 \\
.0005704 \\
.0005415 \\
.0008062\end{array}$ \\
\hline Average.. & 23.5 & 6.84457 & 341.75 & .019779 & 2.88 & .18065 & .0005527 \\
\hline
\end{tabular}

30 TO 40 PER CENT.

\begin{tabular}{|c|c|c|c|c|c|c|c|}
\hline $26101 \ldots \ldots \ldots \ldots$ & $\quad 33.3$ & 1.9790 & 122 & 0.01704 & 3.19 & 0.06318 & 0.0005091 \\
\hline $28201 \ldots \ldots \ldots \ldots$ & 33.3 & 4.3698 & 219 & .01996 & 3.07 & .13415 & .0006126 \\
\hline $28801 \ldots \ldots \ldots$ & 33.3 & 8.3240 & 386 & .02311 & 2.96 & .25019 & .0006842 \\
\hline $33901 \ldots \ldots \ldots$ & 33.3 & 6.7169 & 313 & .02057 & 2.21 & .12186 & .0004466 \\
\hline $37901 \ldots \ldots \ldots$ & 33.3 & .5757 & 28 & .01820 & 2.48 & .01447 & .0004556 \\
\hline $38501 \ldots \ldots \ldots$ & 37.5 & 5.03306 & 252 & .01814 & 3.25 & .24284 & .1006738 \\
\hline $38701 \ldots \ldots \ldots$ & 33.3 & 7.2545 & 365 & .01988 & 2.59 & .18789 & .0005148 \\
\hline $48301 . \ldots \ldots \ldots$ & 33.3 & 7.3424 & 315 & .02117 & 3.08 & .21633 & .0006433 \\
\hline $50901 \ldots \ldots \ldots$ & 33.3 & 2.0631 & 167 & .01000 & 3.43 & .07041 & .0004496 \\
\hline $59601 \ldots \ldots \ldots$ & 33.3 & 8.4456 & 474 & .01796 & 2.14 & .18039 & .0003842 \\
\hline $69701 \ldots \ldots \ldots$ & 33.3 & 3.7810 & 244 & .01550 & 2.50 & .09453 & .0003874 \\
\hline $88901 \ldots . . . .$. & 33.3 & 7.6051 & 419 & .01812 & 2.74 & .20632 & .0004966 \\
\hline $92301 \ldots \ldots \ldots \ldots$ & 33.3 & 4.1975 & 253 & .01611 & 3.93 & .18308 & .0006454 \\
\hline Average... & 33.6 & 5.2065 & 273.6 & .01813 & 2.89 & .15125 & .0005310 \\
\hline
\end{tabular}

40 TO 50 PER CENT.

\begin{tabular}{|c|c|c|c|c|c|c|c|}
\hline $17501 \ldots \ldots \ldots \ldots$ & 42.9 & 1.1495 & 55 & 0.01865 & 4.01 & 0.04268 & 0.0007259 \\
\hline $21301 \ldots \ldots \ldots$ & 44.4 & 4.6950 & 259 & .01819 & 3.01 & .14144 & .0000449 \\
\hline $33101 \ldots \ldots \ldots$ & 42.9 & 2.9905 & 156 & .01858 & 2.73 & .07566 & .0005066 \\
\hline $44601 \ldots \ldots \ldots$ & 42.9 & 1.8251 & 93 & .01963 & 2.73 & .04998 & .0005390 \\
\hline $50701 \ldots \ldots \ldots$ & 40.0 & .5329 & 32 & .01664 & 3.17 & .01712 & $.00 \cup 5396$ \\
\hline $72401 \ldots \ldots \ldots \ldots$ & 40.0 & 8.3672 & 321 & .02946 & 3.15 & .26913 & .0009502 \\
\hline $72801 \ldots \ldots \ldots \ldots$ & 40.0 & 2.0970 & 110 & .01906 & 3.01 & .06312 & .0005738 \\
\hline $72901 \ldots \ldots$ & 40.0 & 2.6462 & 167 & .01585 & 2.48 & .06563 & .0003930 \\
\hline $76201 \ldots \ldots \ldots$ & 40.0 & 6.9409 & 472 & .01456 & 3.40 & .22024 & .0004700 \\
\hline $81401 \ldots \ldots \ldots$ & 40.0 & 2.9064 & 156 & .01791 & $\begin{array}{l}2.70 \\
2.96\end{array}$ & .07905 & .0005288 \\
\hline $86101 \ldots \ldots . . .6$ & 40.0 & 5. 3261 & 314 & .01622 & 2.59 & .14008 & .0004261 \\
\hline $92201 \ldots \ldots$ & 44.4 & 4. 1705 & 238 & .01894 & 2.67 & .11199 & .0005053 \\
\hline $92501 \ldots \ldots \ldots$ & 42.9 & 5. 4034 & 297 & .01771 & 3.32 & .16649 & .0005828 . \\
\hline $94401 \ldots \ldots$ & 42.9 & 8.6610 & 484 & .01769 & 2.27 & .20040 & .0004046 \\
\hline Average.... & 41.7 & 4.1223 & 225.3 & .01843 & 2.96 & .11736 & .0005493 \\
\hline
\end{tabular}


TABLE 29.-Y ields of plants, arranged according to percentage killed in each family-Cont'd.

50 TO 60 PER CENT.

\begin{tabular}{|c|c|c|c|c|c|c|c|}
\hline $\begin{array}{l}\text { Record number } \\
\text { for } 190^{\circ} \text {. }\end{array}$ & $\begin{array}{c}\text { Percent- } \\
\text { age of } \\
\text { plants } \\
\text { in } 1903 \\
\text { surviv- } \\
\text { ing from } \\
1902 .\end{array}$ & $\begin{array}{l}\text { Weight } \\
\text { of ker- } \\
\text { nels on } \\
\text { plant } \\
\text { (gram). }\end{array}$ & $\begin{array}{c}\text { Num- } \\
\text { ber of } \\
\text { kernels. }\end{array}$ & $\begin{array}{l}\text { Weight } \\
\text { of aver- } \\
\text { age } \\
\text { kernel } \\
\text { (gram). }\end{array}$ & $\begin{array}{c}\text { Percent- } \\
\text { age of } \\
\text { proteid } \\
\text { nitrogen } \\
\text { in ker- } \\
\text { nels. }\end{array}$ & $\begin{array}{c}\text { Proteid } \\
\text { nitrogen } \\
\text { in ker- } \\
\text { nels } \\
\text { (gram). }\end{array}$ & $\begin{array}{c}\text { Proteid } \\
\text { nitrogen } \\
\text { in a verage } \\
\text { kernel } \\
(\text { gram). }\end{array}$ \\
\hline $17301 \ldots \ldots \ldots \ldots$ & 50.0 & 3.0000 & 156 & 0.01980 & 3.21 & 0.09556 & 0.0006380 \\
\hline $17401 \ldots \ldots \ldots \ldots$ & 54.5 & 11.7777 & 581 & .01961 & 2.65 & .30061 & .0005161 \\
\hline $20701 \ldots \ldots$ & 50.0 & 6. 6626 & 327 & .02012 & 2.85 & .18906 & $.0005 € .97$ \\
\hline $27201 \ldots \ldots$ & 50.0 & 12.9727 & 611 & .02105 & 2.56 & .31509 & .0005371 \\
\hline $33401 \ldots \ldots \ldots$. & 50.0 & 5.2333 & $27 \mathrm{r}$ & .01818 & 1.98 & .10621 & 0003569 \\
\hline $33601 \ldots . . . .$. & 50.0 & 6.0463 & 273 & .02205 & 2.61 & .14759 & .0005729 \\
\hline $34201 \ldots \ldots \ldots$ & 57.1 & 6.8220 & 328 & .02019 & 2.86 & .18949 & .0005769 \\
\hline $37701 \ldots \ldots \ldots$ & 50.0 & 4.1993 & 237 & .01946 & 2.64 & .12164 & .0005130 \\
\hline $39501 \ldots \ldots \ldots \ldots$ & 50.0 & 1.9040 & 89 & .02284 & 2.97 & .05663 & .0006768 \\
\hline $45601 \ldots \ldots$ & 50.0 & 2.3719 & 140 & .01497 & 2.36 & .04852 & .0003388 \\
\hline $46101 \ldots \ldots$ & 50.0 & 4.8728 & 273 & .01832 & 2.69 & .13084 & .0004924 \\
\hline $55201 \ldots \ldots \ldots \ldots$ & 50.0 & 6.0242 & 309 & .01844 & 2.83 & .15608 & .0005186 \\
\hline $57 \mathrm{f} 01 \ldots \ldots \ldots$ & 57.1 & 9.3804 & 435 & .02178 & 2.37 & .18680 & .0005150 \\
\hline $63101 \ldots \ldots \ldots . . .$. & 50.0 & 4.7193 & 224 & .01984 & 2.82 & .12281 & .0005523 \\
\hline $69801 \ldots \ldots . .$. & 50.0 & 7.2278 & 334 & .02186 & 3.74 & .17078 & .0008247 \\
\hline $85201 \ldots \ldots \ldots$. & 50.0 & 4. 2040 & 295 & .01468 & 2.63 & .11078 & .0003778 \\
\hline $88 c 01 \ldots . . . . . .$. & 57.1 & 5.6295 & 266 & .02236 & 2.57 & .14178 & .0005366 \\
\hline Average.... & 51.5 & 6.0616 & 302.9 & .01974 & 2.73 & .15237 & .0005361 \\
\hline
\end{tabular}

60 TO 70 PER CENT.

\begin{tabular}{|c|c|c|c|c|c|c|c|}
\hline $21201 \ldots \ldots \ldots \ldots$ & 66.7 & 2.5064 & 137 & 0.01956 & 3.57 & 0.09431 & 0.0006846 \\
\hline $32201 \ldots \ldots \ldots$ & 60.0 & 5. 8304 & 288 & .01937 & 2.64 & .11603 & .0005027 \\
\hline $32601 \ldots . . . . .$. & 66.7 & 2.9653 & 166 & .01890 & 2.62 & .05309 & .0006177 \\
\hline $48101 \ldots \ldots$ & 66.7 & 11.6655 & 608 & .01919 & 2.38 & 27765 & .0004567 \\
\hline 48501 & 66.7 & 6.0446 & 341 & .01813 & 3.06 & 18124 & .0005437 \\
\hline $55001 \ldots \ldots \ldots$ & 66.7 & 8.6833 & 476 & .01824 & 3.25 & .25347 & .0005928 \\
\hline $55301 \ldots$ & 66.7 & 5.4606 & 280 & .01874 & 2.27 & 12536 & 0004328 \\
\hline $55 j 01 \ldots$ & 66.7 & 10.4714 & 529 & .01914 & 2.85 & 29155 & .0005428 \\
\hline $57001 \ldots \ldots \ldots$ & 60.0 & 5.0125 & 319 & .01725 & 2.71 & .13688 & .0004658 \\
\hline $57301 \ldots \ldots \ldots$ & 66.7 & 7.7761 & 443 & .01752 & 2.54 & .20018 & .0004588 \\
\hline $57401 \ldots \ldots \ldots$ & 66.7 & 7.6312 & 383 & .01973 & 2.49 & .19910 & .0004900 \\
\hline $57501 \ldots \ldots \ldots$ & 66.7 & 8.1116 & 382 & .02099 & 2.60 & .20327 & .0005320 \\
\hline $63501 \ldots \ldots \ldots$ & 60.0 & 4. 1723 & 229 & .01791 & 2.17 & .06748 & .0003749 \\
\hline $66001 \ldots \ldots \ldots$ & 66.7 & 5.9586 & 309 & .01919 & 3.25 & .17590 & .0005924 \\
\hline $72601 \ldots \ldots \ldots$ & 60.0 & 4. 6412 & 265 & .01758 & 4.04 & .14328 & .0007214 \\
\hline $72701 \ldots$ & 66.7 & 9.3629 & 396 & .02245 & 2.94 & .28276 & .0006629 \\
\hline $73301 \ldots \ldots \ldots \ldots$ & 66.7 & 7.7977 & 354 & .02194 & 2.59 & .21334 & .0005639 \\
\hline $74601 \ldots \ldots \ldots \ldots$ & 60.0 & 8.3679 & 451 & .01854 & 2.49 & .20681 & .0004589 \\
\hline $84901 \ldots . . \ldots \ldots$ & 66.7 & 4. 1284 & 209 & .01951 & 2.87 & .13763 & .0005622 \\
\hline $92901 \ldots . . . . .$. & 62.5 & 4.6818 & 258 & .01763 & 2.81 & .12877 & .0004908 \\
\hline $95701 \ldots \ldots \ldots$ & 60.0 & 5.4211 & 318 & .01672 & 2.58 & .14079 & .0004336 \\
\hline Average.... & 64.6 & 6.5092 & 340 & .01896 & 2.80 & .17280 & .0005324 \\
\hline
\end{tabular}

70 PER CENT AND OVER.

\begin{tabular}{|c|c|c|c|c|c|c|c|}
\hline $21701 \ldots \ldots \ldots$ & 87.5 & 9.75524 & 447 & 0.02157 & 2.78 & 0.30200 & $0.000<049$ \\
\hline $21801 \ldots \ldots \ldots$ & 80.0 & 11.5721 & 622 & .01963 & 3. 13 & .35575 & .0006057 \\
\hline $21901 \ldots \ldots$ & 88.9 & 8.3406 & 398 & .02114 & 3.53 & .30995 & .0007501 \\
\hline $22201 \ldots \ldots \ldots$ & 87.5 & 4.0677 & 229 & .01674 & 3.16 & $.12 f .04$ & .0005292 \\
\hline $26801 \ldots . . . . .$. & 80.0 & 7.1981 & 329 & .02045 & 2.82 & .20306 & .0005768 \\
\hline $26901 \ldots \ldots$ & 71.4 & 3.8910 & 206 & .01871 & 2.77 & .10870 & .0005189 \\
\hline $27301 \ldots \ldots \ldots \ldots$ & 80.0 & 6.6162 & 343 & .01913 & 2.93 & .18438 & .0005447 \\
\hline $27501 \ldots \ldots$ & 71.4 & 6.8618 & 310 & .02152 & 2.69 & .17267 & .0005758 \\
\hline $38601 \ldots \ldots \ldots \ldots$ & 71.4 & 3.9532 & 186 & .01983 & 3.72 & .11558 & .0007264 \\
\hline $48401 \ldots \ldots \ldots$. & 83.3 & 4. 4668 & 277 & .01502 & 2.90 & .10033 & .0004159 \\
\hline $55601 \ldots \ldots$. & 83.3 & 10.2785 & 435 & .02211 & 2.55 & .29008 & .0005589 \\
\hline $55901 \ldots$ & 83.3 & 10.9242 & 489 & .02234 & 2.56 & .27788 & .0005632 \\
\hline $56101 \ldots$ & 75.0 & 10.7383 & 617 & .01744 & 2.75 & .29783 & .0004790 \\
\hline $56201 \ldots$ & 83.3 & 11.2241 & 563 & .02034 & 2.49 & .27997 & .0005065 \\
\hline $58201 \ldots \ldots$. & 75.0 & 2.8084 & 227 & .01159 & 2.88 & .08385 & 0003383 \\
\hline $65301 \ldots \ldots \ldots \ldots$ & 83.3 & 7.5858 & 394 & .02001 & 2.92 & .18248 & $.000 t 050$ \\
\hline $74501 \ldots \ldots \ldots$ & 100.0 & 3.4799 & 191 & .01695 & 2.78 & .10355 & .0004745 \\
\hline $81701 \ldots \ldots \ldots$ & 100.0 & 12.7593 & 569 & .02272 & 2.27 & .29500 & .0005170 \\
\hline $92401 \ldots \ldots \ldots \ldots$ & 71.4 & 4. 4131 & 234 & .01822 & 2. 63 & .12426 & .0004826 \\
\hline $94201 \ldots \ldots \ldots$. & 83.3 & 5.9603 & 339 & .01748 & 2.58 & .16548 & .0004426 \\
\hline $94901 \ldots \ldots$. & 75.0 & 7.0172 & 388 & .01780 & 2.85 & .21294 & .0005072 \\
\hline $95501 \ldots \ldots \ldots \ldots$ & 100.0 & 7.2956 & 374 & .01767 & 2.50 & .18689 & .0004118 \\
\hline A yerage. & 82.4 & 7.3275 & 371.2 & .01902 & 2.83 & .20357 & .0005348 \\
\hline
\end{tabular}


TABLE 30.-Summary of yields of plants, arranged according to percentage killed in each family.

\begin{tabular}{|c|c|c|c|c|c|c|c|c|}
\hline \multirow{2}{*}{$\begin{array}{l}\text { Percentage of plants } \\
\text { grouped according } \\
\text { to survivors of } 1903 \\
\text { from } 1902 .\end{array}$} & \multirow{2}{*}{$\begin{array}{l}\text { Num- } \\
\text { ber of } \\
\text { analy- } \\
\text { ses. }\end{array}$} & \multirow{2}{*}{$\begin{array}{l}\text { Percent- } \\
\text { age of } \\
\text { plants in } \\
1903 \text { sur- } \\
\text { viving } \\
\text { from } 1902 .\end{array}$} & \multirow{2}{*}{$\begin{array}{l}\text { Weight } \\
\text { of ker- } \\
\text { nels on } \\
\text { plant } \\
\text { (grams). }\end{array}$} & \multirow{2}{*}{$\begin{array}{c}\text { Num- } \\
\text { ber of } \\
\text { kernels } \\
\text { per } \\
\text { plant. }\end{array}$} & \multirow{2}{*}{$\begin{array}{l}\text { Weight } \\
\text { of aver- } \\
\text { age ker- } \\
\text { nel } \\
\text { (gram). }\end{array}$} & \multirow{2}{*}{$\begin{array}{l}\text { Percent- } \\
\text { age of } \\
\text { proteid } \\
\text { nitrogen } \\
\text { in ker- } \\
\text { nels. }\end{array}$} & \multicolumn{2}{|c|}{$\begin{array}{l}\text { Proteid nitrogen } \\
\quad(\text { gram) in- }\end{array}$} \\
\hline & & & & & & & Kernels. & $\begin{array}{l}\text { Average } \\
\text { kernel. }\end{array}$ \\
\hline $\begin{array}{l}10 \text { to } 20 \ldots \ldots \\
20 \text { to } 30 \ldots \ldots \\
30 \text { to } 40 \ldots \ldots \\
40 \text { to } 50 \ldots \ldots \\
50 \text { to } 60 \ldots \ldots \\
60 \text { to } 70 \ldots \ldots \\
70 \text { and over } \ldots\end{array}$ & $\begin{array}{l}30 \\
20 \\
13 \\
14 \\
17 \\
21 \\
22\end{array}$ & $\begin{array}{l}15.8 \\
23.5 \\
33.6 \\
41.7 \\
51.5 \\
64.6 \\
82.4\end{array}$ & $\begin{array}{l}4.7098 \\
6.8446 \\
5.2065 \\
4.1223 \\
6.0616 \\
6.5092 \\
7.3275\end{array}$ & $\begin{array}{l}251 \\
342 \\
274 \\
225 \\
303 \\
340 \\
371\end{array}$ & $\begin{array}{r}0.01856 \\
.01978 \\
.01813 \\
.01843 \\
.01974 \\
.01896 \\
.01902\end{array}$ & $\begin{array}{l}2.91 \\
2.88 \\
2.89 \\
2.96 \\
2.73 \\
2.80 \\
2.83\end{array}$ & $\begin{array}{r}0.12294 \\
.18065 \\
.15125 \\
.11736 \\
.15237 \\
.17280 \\
.20357\end{array}$ & $\begin{array}{r}0.0005437 \\
.0005527 \\
.0005310 \\
.0005493 \\
.0005361 \\
.0005324 \\
.0005348\end{array}$ \\
\hline
\end{tabular}

\section{YIELD AND NITROGEN CONTENT OF GRAIN AS AFFECTED BY LENGTH OF GROWING PERIOD.}

Early-maturing varieties of wheat are, in general, better yielding sorts in Nebraska than are later maturing ones. There are some exceptions to this rule, however, Turkish Red yielding better than any earlier maturing variety. The advantages from early maturity have usually been ascribed to the cooler weather and greater supply of moisture that obtain in the early summer. The hot, dry weather common in July is thought to prevent the filling out of the kernel and to cause light yield and light volume weight.

Each wheat plant on the breeding plots was harvested separately in 1903, and a record was kept of the date of harvesting of each of these plants. These data have been tabulated for the purpose of showing the relation between the length of the growing season and the yield of grain from individual plants of the same variety.

Table 31 contains these data, tabulated according to the date of ripening. Plants ripening between the 7th and 11th of July, 1903, form the first class, those ripening between July 11 and 15 the second class, and the succeeding classes increase by four days until July 27 , all ripening after that date constituting the last class. The dates of ripening thus extend over a period of three weeks.

The season of 1903 was a very wet and cool one. The effect of this upon the wheat crop is shown by the fact that the crop in the field was not ready to harvest until July 10, while usually it is harvested between the 20th and 30th of June. Even at the close of the ripening period the weather did not become dry or hot as compared with the normal season. It will therefore be seen that the ordinary advantages from early maturity did not obtain, or at least not in the customary way. It may also be said that some of the later maturing wheats yielded as well in 1904 as did the Turkish Red.

Table 32 is a summary of Table 31 , with a statement of the average for each class.

Table 33 is a summary of the same plants, tabulated according to the yield of grain per plant. 
Table 34 is a summary of the same plants, tabulated according to the percentage of proteid nitrogen.

It is very evident from these tables that the early-maturing plants are the most prolific. The weight of the average kernel remains very uniform, so that the later maturing plants do not appear to have produced shrunken kernels. Evidently the plants ripening during the first four days produced the largest amounts of grain, and their kernels were as heavy as those produced later. The smaller productiveness of the later maturing plants in the season of 1903 does not appear to have been due to a shrunken or light kernel.

The percentage of proteid nitrogen appears to be somewhat less in the grain of the early-maturing plants. The number of grams of proteid nitrogen in the average kernel is likewise less in these earlymaturing plants.

The relation of length of growing season to both yield and composition of grain is contrary to what might have been supposed. A long growing period without excessively hot or dry weather might naturally be thought to increase the yield and increase the percentage of carbohydrates in the grain.

The season of 1904 was very similar to that of 1903 up to time of wheat harvest. The data for 1904, when tabulated, will serve as a check on the results obtained in 1903.

TABLE 31.- Yield and nitrogen content of grain, tabulated according to length of growing period.

DATES RIPE: JULY 7 TO 11, 1903.

\begin{tabular}{|c|c|c|c|c|c|c|}
\hline \multirow{2}{*}{ Record number. } & \multirow{2}{*}{$\begin{array}{l}\text { Date } \\
\text { ripe. }\end{array}$} & \multirow{2}{*}{$\begin{array}{c}\text { Yield } \\
\text { (grams). }\end{array}$} & \multirow{2}{*}{$\begin{array}{l}\text { Percent- } \\
\text { age of } \\
\text { proteid } \\
\text { nitrogen. }\end{array}$} & \multirow{2}{*}{$\begin{array}{c}\text { Weight } \\
\text { of aver- } \\
\text { age ker- } \\
\text { nel } \\
\text { (gram). }\end{array}$} & \multicolumn{2}{|c|}{$\begin{array}{l}\text { Proteid nitrogen } \\
\quad(\text { gram) in- }\end{array}$} \\
\hline & & & & & Kernels. & $\begin{array}{l}\text { A verage } \\
\text { kernel. }\end{array}$ \\
\hline 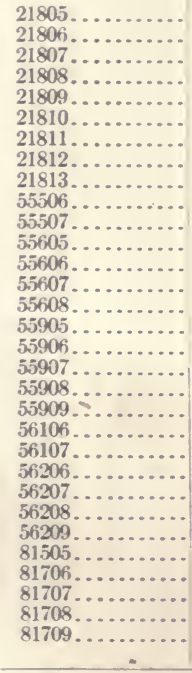 & 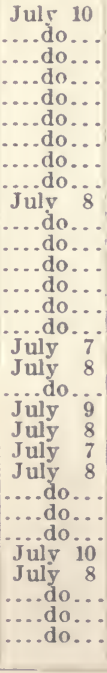 & $\begin{array}{r}20.9290 \\
14.2450 \\
9.4172 \\
19.7446 \\
8.0214 \\
1.0304 \\
11.9114 \\
14.8139 \\
4.0258 \\
17.8506 \\
9.8228 \\
10.9180 \\
11.0930 \\
2.3931 \\
22.5848 \\
5.7948 \\
7.9968 \\
19.3966 \\
12.1221 \\
9.2120 \\
12.0161 \\
14.4556 \\
9.3093 \\
10.9073 \\
13.5720 \\
15.8086 \\
2.8327 \\
15.3928 \\
18.3614 \\
7.3993 \\
16.4692\end{array}$ & $\begin{array}{l}2.69 \\
2.71 \\
2.73 \\
2.57 \\
2.73 \\
2.69 \\
3.75 \\
4.26 \\
4.04 \\
2.80 \\
2.63 \\
2.64 \\
2.58 \\
2.69 \\
2.31 \\
2.67 \\
2.81 \\
2.59 \\
2.42 \\
2.30 \\
2.57 \\
2.96 \\
2.42 \\
2.34 \\
2.61 \\
2.59 \\
2.94 \\
2.71 \\
2.34 \\
2.41 \\
2.28\end{array}$ & $\begin{array}{l}0.01699 \\
.02378 \\
.02498 \\
.01708 \\
.01919 \\
.019816 \\
.021007 \\
.01507 \\
.01877 \\
.02062 \\
.01949 \\
.02184 \\
.02205 \\
.01734 \\
.02699 \\
.01751 \\
.01603 \\
.02590 \\
.02175 \\
.03050 \\
.01866 \\
.01658 \\
.01829 \\
.02361 \\
.02356 \\
.01664 \\
.01940 \\
.02132 \\
.02336 \\
.02578 \\
.02175\end{array}$ & $\begin{array}{r}0.56299 \\
.38604 \\
.25709 \\
.50744 \\
.21898 \\
.02772 \\
.44666 \\
.63107 \\
.16377 \\
.49995 \\
.25834 \\
.28823 \\
.28580 \\
.06437 \\
.52194 \\
.15470 \\
.22471 \\
.50238 \\
.29575 \\
.21187 \\
.30881 \\
.42790 \\
.22529 \\
.25522 \\
.34616 \\
.40945 \\
.08328 \\
.41715 \\
.42965 \\
.17833 \\
.37548\end{array}$ & $\begin{array}{r}0.0004569 \\
.0006444 \\
.0006664 \\
.0004389 \\
.0005238 \\
.0005330 \\
.0007877 \\
.0006420 \\
.0007582 \\
.0005773 \\
.0005126 \\
.0005765 \\
.0005690 \\
.0004665 \\
.0006236 \\
.0004674 \\
.0004503 \\
.0006707 \\
.0005262 \\
.0007016 \\
.0004795 \\
.0004907 \\
.0004426 \\
.0005524 \\
.0006149 \\
.0004310 \\
.0005704 \\
.0005778 \\
.0005466 \\
.0006213 \\
.0004960 \\
\end{array}$ \\
\hline
\end{tabular}


TABLE 31.-Yield and nitrogen content of grain, tabulated according to length of growing period-Continued.

DATES RIPE: JULY 7 TO 11, 1903-Continued.

\begin{tabular}{|c|c|c|c|c|c|c|}
\hline \multirow{2}{*}{ Record number. } & \multirow{2}{*}{$\begin{array}{l}\text { Date } \\
\text { ripe. }\end{array}$} & \multirow{2}{*}{$\begin{array}{c}\text { Yield } \\
\text { (grams). }\end{array}$} & \multirow{2}{*}{$\begin{array}{l}\text { Percent- } \\
\text { age of } \\
\text { proteid } \\
\text { nitrogen. }\end{array}$} & \multirow{2}{*}{$\begin{array}{l}\text { Weight } \\
\text { of aver- } \\
\text { age ker- } \\
\text { nel } \\
\text { (gram). }\end{array}$} & \multicolumn{2}{|c|}{$\begin{array}{l}\text { Proteid nitrogen } \\
\text { (gram) in- }\end{array}$} \\
\hline & & & & & Kernels. & $\begin{array}{l}\text { Average } \\
\text { kernel. }\end{array}$ \\
\hline 81710 & July 8 & 9.1411 & 1.92 & 0.02308 & 0.17550 & 0.0004432 \\
\hline $88605 \ldots \ldots$ & July 10 & 1.6362 & 2.80 & .02731 & .04581 & .0007640 \\
\hline $88606 \ldots \ldots$ & ....do... & 9.9456 & 2.53 & .02068 & .25162 & .0005231 \\
\hline $88607 \ldots .$. & ... do... & 5.1584 & 2.61 & .02205 & .13463 & .0005754 \\
\hline $88608 \ldots .$. & ....do... & 1.5355 & 2.47 & .02075 & .03793 & .0005125 \\
\hline $88609 \ldots .$. & ...do... & 9.8719 & 2.42 & .02100 & .23890 & .0005082 \\
\hline $94907 \ldots$ & ...do... & 12.1918 & 2.94 & .01948 & .35844 & .0005726 \\
\hline $94908 \ldots \ldots \ldots \ldots$ & ...do... & 2.3678 & 1.96 & .01894 & .04641 & .0003713 \\
\hline $94909 \ldots$ & July 9 & 3. 6977 & 3.60 & .01696 & .13312 & .0006106 \\
\hline $95505 \ldots$ & ....do... & .3146 & 2.81 & .00850 & .00884 & .0002389 \\
\hline $95506 \ldots$ & .... do... & 11.0548 & 2.74 & .01852 & .30291 & .0005074 \\
\hline $95507 \ldots$ & ....do... & 12.1592 & 2.59 & .02029 & .31492 & .0005515 \\
\hline $95508 \ldots$ & ....do... & 14.4617 & 2.56 & .01954 & .37023 & .0005003 \\
\hline $95509 \ldots$ & .... do ... & 2:9475 & 2. 48 & .02136 & .07310 & .0005297 \\
\hline $95510 \ldots$ & .... do ... & 2. 8356 & 1.81 & .01783 & .05132 & .0003228 \\
\hline $95705 \ldots$ & July 10 & 10.3426 & 2.54 & .01626 & .26270 & .0004131 \\
\hline $95706 \ldots$ & .... do ... & 5. 1629 & 2.73 & .01934 & 14095 & .0005279 \\
\hline $95707 \ldots \ldots$ & .... do ... & .7577 & 2.47 & .01457 & .01872 & .0003599 \\
\hline Average ... & July 8.9 & 9.9067 & 2.69 & .02024 & .26475 & .0005356 \\
\hline
\end{tabular}

DATES RIPE: JULY 11 TO 15, 1903.

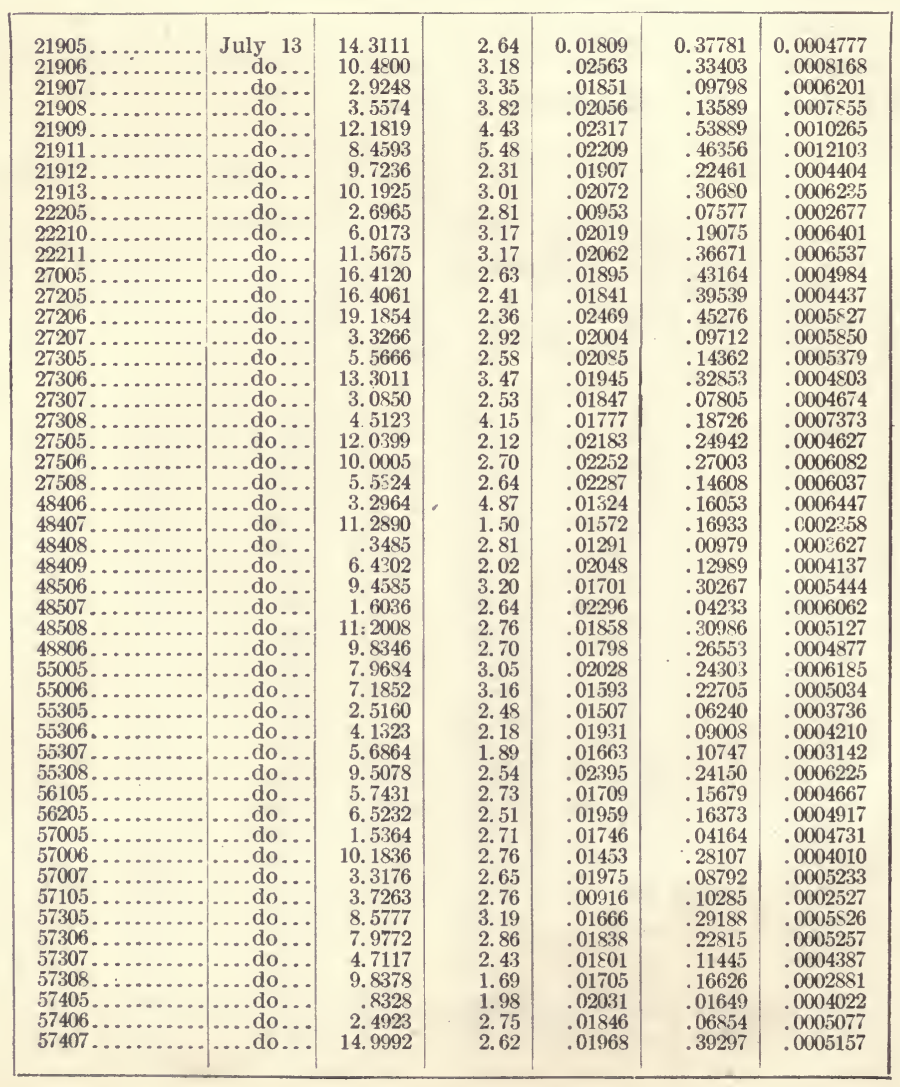


TABLE 31.-Yield aind nitrogen content of grain, tabulated according to length of growing period-Continued.

DATES RIPE: JULY 11 TO 15, 1903-Continued.

\begin{tabular}{|c|c|c|c|c|c|c|}
\hline \multirow{2}{*}{ Record number. } & \multirow{2}{*}{$\begin{array}{l}\text { Date } \\
\text { ripe. }\end{array}$} & \multirow{2}{*}{$\begin{array}{c}\text { Yield } \\
\text { (grams). }\end{array}$} & \multirow{2}{*}{$\begin{array}{c}\text { Percent- } \\
\text { age of } \\
\text { proteid } \\
\text { nitrogen. }\end{array}$} & \multirow{2}{*}{$\begin{array}{c}\text { Weight } \\
\text { of aver- } \\
\text { age ker- } \\
\text { nel } \\
\text { (gram). }\end{array}$} & \multicolumn{2}{|c|}{$\begin{array}{l}\text { Proteid nitrogen } \\
\text { (gram) in- }\end{array}$} \\
\hline & & & & & Kernels. & $\begin{array}{l}\text { Average } \\
\text { kernel. }\end{array}$ \\
\hline 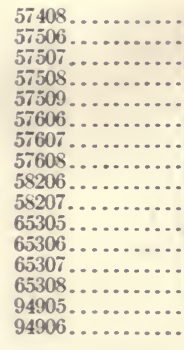 & 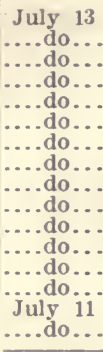 & $\begin{array}{r}12.2004 \\
2.7616 \\
6.9861 \\
12.0728 \\
10.6261 \\
3.0790 \\
16.4433 \\
8.6189 \\
1.3961 \\
4.2207 \\
1.8018 \\
9.8298 \\
7.0051 \\
11.7006 \\
4.4423 \\
12.3862\end{array}$ & $\begin{array}{l}2.61 \\
2.80 \\
2.85 \\
2.21 \\
2.54 \\
2.74 \\
1.73 \\
2.64 \\
2.67 \\
3.09 \\
4.92 \\
2.41 \\
2.28 \\
2.09 \\
2.35 \\
3.41\end{array}$ & $\begin{array}{l}0.02047 \\
.01534 \\
.01946 \\
.03177 \\
.01739 \\
.02333 \\
.02234 \\
.01968 \\
.00943 \\
.01375 \\
.02310 \\
.01807 \\
.01878 \\
.02008 \\
.01553 \\
.01808\end{array}$ & $\begin{array}{l}0.31842 \\
.07733 \\
.19905 \\
.26680 \\
.26990 \\
.08436 \\
.24847 \\
.22756 \\
.03728 \\
.13042 \\
.08865 \\
.23690 \\
.15971 \\
.24468 \\
.10439 \\
.42236\end{array}$ & $\begin{array}{l}0.0005343 \\
.0004296 \\
.0005545 \\
.0007021 \\
.0004417 \\
.0006391 \\
.0003865 \\
.0005195 \\
.0002519 \\
.0004248 \\
.0011365 \\
.0004355 \\
.0004282 \\
.0004197 \\
.0003650 \\
.0006166\end{array}$ \\
\hline A verage.. & July 13 & 7.6611 & 2.81 & .01887 & .20820 & .0005290 \\
\hline
\end{tabular}

DATES RIPE: JULY 15 TO 19, 1903.

\begin{tabular}{|c|c|c|c|c|c|c|}
\hline $18906 \ldots \ldots \ldots$. & July 15 & 0.9229 & 3.48 & 0.01420 & 0.03212 & 0.0004941 \\
\hline $21706 \ldots \ldots \ldots$ & ....do... & 19.3318 & 4.71 & .02390 & .91052 & .0011283 \\
\hline $21707 \ldots \ldots \ldots$ & ....do... & 12.3685 & 2.19 & .02125 & .27086 & .0004654 \\
\hline $26105 \ldots \ldots \ldots$ & ....do... & 1.8242 & 3.02 & .01393 & .05508 & .0003662 \\
\hline $33406 \ldots \ldots \ldots$. & July 18 & 4.6045 & 2.87 & .01627 & .13215 & .0004670 \\
\hline $34206 \ldots \ldots \ldots \ldots$ & ....do... & 1.5940 & 3.73 & .01968 & .05946 & .0007340 \\
\hline $34208 \ldots \ldots \ldots \ldots$ & ....do ... & 2.9886 & 2.13 & .01916 & .06366 & .0004081 \\
\hline $37906 \ldots \ldots \ldots \ldots$ & July 15 & .2062 & 2.44 & .01086 & .00503 & .0002649 \\
\hline $45005 \ldots \ldots \ldots \ldots$ & .... do... & 3.2310 & 3.58 & .01376 & .11575 & .0004927 \\
\hline $45605 \ldots . . . . .$. & ....do... & .7081 & 2.82 & .01161 & .01997 & .0003273 \\
\hline $48405 \ldots \ldots \ldots$. & ....do... & .9701 & 3.31 & .01276 & .03211 & .0004225 \\
\hline $48505 \ldots \ldots \ldots$ & ...do... & 1.9154 & 3.66 & .01398 & .07010 & .0005117 \\
\hline $51005 \ldots \ldots \ldots \ldots$ & $\ldots$... do ... & 15.5835 & 1.34 & .01804 & .20881 & .0002422 \\
\hline $63105 \ldots \ldots \ldots$ & July 18 & 1.5452 & 3.24 & .01717 & .05007 & .0005563 \\
\hline $63106 \ldots \ldots \ldots$ & .... do ... & 3.3006 & 2.79 & .02001 & .09208 & .0005581 \\
\hline $66006 \ldots \ldots \ldots$ & $\ldots$ do.... & 6.0090 & 3.54 & .01642 & .21272 & .0005812 \\
\hline $72605 \ldots \ldots \ldots$ & ....do... & 1.1166 & 4.65 & .01718 & .05192 & .0007988 \\
\hline $72806 \ldots \ldots \ldots$ & ....do... & 2.0970 & 3.01 & .01906 & .06312 & .0005738 \\
\hline $74605 \ldots \ldots \ldots$ & ....do... & 7.1181 & 2.60 & .01784 & .18507 & .0004638 \\
\hline $81705 \ldots \ldots \ldots$ & $\ldots$ do... & 9.7922 & 1.98 & .02106 & .19388 & .0004170 \\
\hline $85905 \ldots . . . . .$. & July 16 & 5. 3069 & 2.83 & .01811 & .15019 & .0005126 \\
\hline $89906 \ldots \ldots \ldots$ & .....do... & 9.9034 & 2.65 & .01814 & .26245 & .0004807 \\
\hline $91905 \ldots \ldots \ldots$ & ....do... & 3.4436 & 3.36 & .01739 & .11570 & .0005844 \\
\hline $91906 \ldots . . . . .$. & ....do.... & 3.5486 & 2.81 & .01774 & .09972 & .0004986 \\
\hline $92205 \ldots \ldots . . .$. & do... & 5.2616 & 2. 74 & .01525 & .14417 & .0004179 \\
\hline $92206 \ldots \ldots \ldots$ & do... & 1.1074 & 2.67 & .02407 & .02957 & .0006428 \\
\hline $92207 \ldots \ldots . . .$. & ....do... & 3.6926 & 2.55 & .01767 & .09416 & .0004505 \\
\hline $92208 \ldots . . . . .$. & $\ldots$ do... & 6.6206 & 2. 72 & .01876 & .18008 & .0005102 \\
\hline $92305 \ldots \ldots \ldots$ & do. & 2.3859 & 2.93 & .01491 & .06991 & .0004369 \\
\hline $92306 \ldots \ldots \ldots$ & do. & 6.0091 & 4.93 & .01732 & .29625 & .0008539 \\
\hline $92406 \ldots \ldots \ldots$ & do. & 8.2366 & 3.11 & .02168 & .25616 & $.00067+1$ \\
\hline $92407 \ldots \ldots . .$. & do. & .8983 & 1.66 & .01695 & 01491 & .0002814 \\
\hline $92408 \ldots \ldots \ldots . .$. & do. & 3.7820 & 2.97 & .01827 & .11233 & .0005426 \\
\hline $92409 \ldots \ldots \ldots$ & do. & 5.7131 & 2.30 & .01814 & .13140 & .0004171 \\
\hline $92506 \ldots \ldots \ldots \ldots$ & do... & 3.8709 & 4. 39 & .01690 & .16993 & .0007421 \\
\hline $92: 07 \ldots \ldots \ldots$ & do... & 9.6779 & 2.58 & .01916 & .24969 & .0004944 \\
\hline $92905 \ldots \ldots \ldots$. & do... & 2.7000 & 3.50 & .01534 & .09450 & .0005369 \\
\hline $92906 \ldots . . . .$. & ....do... & 2.8816 & 2.99 & .01592 & .08616 & .0004760 \\
\hline $92907 \ldots \ldots \ldots \ldots$ & .... do... & 4.4673 & 2.56 & .02040 & .11436 & .0005220 \\
\hline $92908 \ldots \ldots \ldots$ & ....do... & 3.2388 & 2.32 & .01732 & .07514 & .0004018 \\
\hline $92909 \ldots \ldots \ldots$ & ...do... & 10.1363 & 2.70 & .01916 & .27367 & .0005173 \\
\hline $94105 \ldots \ldots \ldots \ldots$ & July 15 & .5595 & 2.67 & .02543 & .01494 & .0006790 \\
\hline $94205 \ldots \ldots \ldots$. & July 16 & 1.2117 & 1.6 .5 & .01893 & .01999 & .0003124 \\
\hline $94206 \ldots . . . . .$. & ....do... & 7.5006 & 2. 78 & .01866 & .208 .51 & .0005187 \\
\hline $94207 \ldots \ldots \ldots \ldots$ & ....do... & 13. 7057 & 2.86 & .01909 & .39199 & .0005460 \\
\hline $94208 \ldots$. & ....do... & 3. 7829 & 3.10 & .01175 & .11727 & .0003642 \\
\hline $91406 \ldots$ & ....do... & 10.5556 & 2.47 & .01923 & .26073 & .0004749 \\
\hline $94407 \ldots$ & ....do... & 6.7664 & 2.07 & .01615 & .14007 & .0003343 \\
\hline $94605 \ldots$ & ....do... & .7319 & 1.95 & .01307 & .01427 & .0002549 \\
\hline $94606 \ldots$ & ....do... & 11.8435 & 1.80 & .07544 & .21319 & .0013576 \\
\hline Average. . & July 16.2 & 5.1354 & 2.87 & .01869 & . 14452 & .0005222 \\
\hline
\end{tabular}


TABLE 31.-Yield and nitrogen content of grain, tabulated according to length of growing period-Continued.

DATES RIPE: JULY 19 TO 23, 1903.

\begin{tabular}{|c|c|c|c|c|c|c|}
\hline \multirow{2}{*}{ Record number. } & \multirow{2}{*}{$\begin{array}{l}\text { Date } \\
\text { ripe. }\end{array}$} & \multirow{2}{*}{$\begin{array}{c}\text { Yield } \\
\text { (grams). }\end{array}$} & \multirow{2}{*}{$\begin{array}{c}\text { Percent- } \\
\text { age of } \\
\text { proteid } \\
\text { nitrogen. }\end{array}$} & \multirow{2}{*}{$\begin{array}{l}\text { Weight } \\
\text { of aver- } \\
\text { age ker- } \\
\text { nel } \\
\text { (gram). }\end{array}$} & \multicolumn{2}{|c|}{$\begin{array}{l}\text { Proteid nitrogen } \\
\quad \text { (gram) in- }\end{array}$} \\
\hline & & & & & Kernels. & $\begin{array}{l}\text { Average } \\
\text { kernel. }\end{array}$ \\
\hline 17409. & July 21 & 14.8957 & 2.75 & 0.01857 & 0.40964 & 0.0005108 \\
\hline 17505 & July 20 & .3885 & 4.70 & .01340 & $\begin{array}{r}.01826 \\
.018\end{array}$ & .0006296 \\
\hline $18805 \ldots \ldots$ & July 21 & 2.1462 & 2.02 & .01567 & .04335 & .0003164 \\
\hline 20707 . & ...do... & 9.9070 & 2.77 & .02282 & .27443 & .0006181 \\
\hline 20708 & July 20 & 2.4690 & 2.58 & .02024 & .06399 & .0005221 \\
\hline 21211 & July 21 & .2806 & 3.15 & .02806 & .00884 & .0008839 \\
\hline 21306 & July 20 & 4. 1516 & 2.90 & .01837 & .12039 & .0005327 \\
\hline 21308 & .... do... & 5.8080 & 3.45 & .01641 & .20038 & .0005660 \\
\hline 21710 & July 21 & .8178 & 2.59 & .01437 & .02196 & .0003722 \\
\hline 21711 . & ....do... & 17.1820 & 2.71 & .01968 & .46563 & .0005334 \\
\hline 22209 & ... do... & .4336 & 3.84 & .01399 & 01665 & .0005371 \\
\hline $26806 \ldots$ & July 20 & 2.7255 & 2.60 & .01793 & .07086 & .0004662 \\
\hline $26807 \ldots \ldots$ & ....do... & 17. 2324 & 2.80 & .02390 & .48250 & .0006692 \\
\hline $26808 \ldots \ldots \ldots$ & ... do... & 3.8811 & 3.09 & .01748 & .11992 & .0005402 \\
\hline $26906 \ldots . .$. & July 22 & 4. 2376 & 2.71 & .01859 & .11484 & .0005037 \\
\hline $26907 \ldots \ldots$ & July 20 & 1.8276 & 2.61 & .01792 & .04995 & .0004677 \\
\hline $26909 \ldots \ldots$ & .... do... & 2.9999 & 2.80 & .01667 & .08400 & .0004667 \\
\hline $32606 \ldots \ldots$ & July 22 & 2.0162 & 2.88 & .02145 & .05807 & .0006177 \\
\hline $33105 \ldots \ldots$ & July 21 & 2.5601 & 2.91 & .01939 & .07450 & .0005644 \\
\hline $33905 \ldots \ldots \ldots$ & .... do... & 11.1476 & 1.61 & .02194 & .17948 & .0003533 \\
\hline $33906 \ldots \ldots \ldots$ & .... do... & 2.2862 & 2.81 & .01921 & .06424 & .0005399 \\
\hline $38606 \ldots \ldots$ & ....do... & 8.4605 & 2.63 & .02110 & .22251 & .0005549 \\
\hline $38607 \ldots$ & ....do... & .3037 & 4.55 & .01598 & .01382 & .0007273 \\
\hline $38608 \ldots . .$. & ....do... & 3.0228 & 2.82 & .01913 & .08522 & .0005394 \\
\hline $38609 \ldots \ldots$ & .... do .... & 6. 7665 & 2.74 & .02319 & .18540 & .0006475 \\
\hline $38706 \ldots$ & July 20 & 7.2545 & 2.59 & .01988 & .18789 & .0005148 \\
\hline $40405 \ldots \ldots$ & July 21 & .6316 & 3.17 & .01373 & .02002 & .0004352 \\
\hline $42206 \ldots \ldots \ldots$ & ....do... & .3161 & 1.46 & .01264 & .00462 & .0001846 \\
\hline $44607 \ldots \ldots$ & July 20 & 1.8246 & 2.44 & .01806 & .04452 & .0004408 \\
\hline $48106 \ldots .$. & July 21 & 11.6655 & 2.38 & .01919 & .27765 & .0004567 \\
\hline $48305 \ldots \ldots$ & July 20 & 12.0278 & 2.87 & .02543 & .34524 & .0007299 \\
\hline $48306 \ldots \ldots$ & ....do... & 2. 6571 & 3.29 & .01692 & .08742 & .0005568 \\
\hline $48706 \ldots$ & ...do... & 6.1989 & 3.00 & .01635 & .18596 & .0004906 \\
\hline 55007 . & ....do... & 2.1571 & 4.21 & .01828 & .09082 & .0007696 \\
\hline $55008 \ldots$ & July 21. & 17.4226 & 2.60 & .01846 & .45299 & .0004799 \\
\hline $55206 \ldots \ldots \ldots$ & ....do... & 11. 3592 & 2.56 & .01965 & .29079 & .0005031 \\
\hline $58805 \ldots \ldots \ldots$ & July 20 & 23.1471 & 2.74 & .01999 & .63422 & .0005464 \\
\hline $59606 \ldots \ldots$ & ...do... & 9.7084 & 2.16 & .01712 & .20970 & .0003698 \\
\hline $63107 \ldots \ldots$ & ....do.... & 9.3120 & 2.43 & .02233 & .22628 & .0005426 \\
\hline $63505 \ldots \ldots \ldots$ & July 21 & 4.0230 & 1.90 & .01934 & $: 07644$ & .0003674 \\
\hline $66008 \ldots$ & July 20 & 3.1555 & 3.59 & .01814 & .11328 & .0006510 \\
\hline $69305 \ldots$ & ....do... & 2.0430 & 4.42 & .01984 & .09030 & .0008767 \\
\hline $71905 \ldots$ & ....do... & 28.2136 & 2.47 & .02239 & .69688 & .0005531 \\
\hline $72606 \ldots$ & ....do... & 9.3629 & 1.89 & .01724 & .18538 & .0003414 \\
\hline 72607 & $\ldots$ do... & 3.4442 & 5.59 & .01832 & .19253 & .0010241 \\
\hline 72705 & ...do... & 9.1522 & 2.13 & .02191 & .19936 & .0004668 \\
\hline 72706 . & ...do... & 14.6802 & 3.86 & .02484 & .56666 & .0009588 \\
\hline 72707 . & July 21 & 4.5806 & 3.49 & .02036 & .15986 & .0007105 \\
\hline 72708 . & July 20 & 9. 0386 & 2.27 & .02270 & .20518 & .0005154 \\
\hline 74507 . & July 21 & 9.2130 & 3.02 & .01869 & .27823 & .0005644 \\
\hline 76206 & July 20 & 5.4411 & 4.45 & .01217 & .24213 & .0005417 \\
\hline 84905 . & .... do... & .7130 & 2.32 & .01927 & .01654 & .0004471 \\
\hline 84906 . & ...do... & 7.5438 & 3.43 & .01975 & .25873 & .0006773 \\
\hline 85206 . & July 21 & 4.9315 & 2. 66 & .01312 & .13118 & .0003332 \\
\hline 92405 . & ....do... & 3.4356 & 3.10 & .01605 & .10650 & .0004977 \\
\hline 94209. & do & 3.6006 & 2.49 & .01895 & .08965 & .0004719 \\
\hline Average. - & July 20.1 & 6.5399 & 2.93 & .01886 & .18064 & .0005482 \\
\hline
\end{tabular}

DATES RIPE: JULY 23 TO 27, 1903.

\begin{tabular}{|c|c|c|c|c|c|c|}
\hline 17305. & July 23 & 3. 6302 & 3.03 & 0.01984 & 0.10999 & 0.0006010 \\
\hline $17306 \ldots . . . .$. & ....do... & 3.9968 & 3.09 & .01645 & $\because 12350$ & .0005082 \\
\hline $17308 \ldots \ldots \ldots$ & ....do... & 1.2275 & 3.25 & .02012 & .03994 & .0006540 \\
\hline $17406 \ldots$. & ....do... & 2.0907 & 3.29 & .01686 & .06878 & .0005547 \\
\hline $17408 \ldots \ldots$ & ....do... & 9.2038 & 2.18 & .01852 & .20065 & .0004037 \\
\hline $17410 \ldots$ & ....do... & 16.9987 & 2.88 & .02285 & .48957 & .0006580 \\
\hline $20705 \ldots$ & ....do.... & 1.8517 & 3.09 & .01698 & .05722 & .0005249 \\
\hline 20706 & ....do... & 3.3138 & 2.78 & .02033 & .09212 & .0005652 \\
\hline 20710 . & ....do... & 17.1115 & 2.83 & .01974 & .48428 & .0005586 \\
\hline 20805 . & ....do... & 14.6942 & 3.32 & .02157 & .48784 & .0006999 \\
\hline
\end{tabular}


YIELD, ETC., AS AFFECTED BY GROWING PERIOD.

TABLE 31.-Yield and nitrogen content of grain, tabulated according to length of growing period-Continued.

DATES RIPE: JULY 23 TO 27, 1903-Continued.

\begin{tabular}{|c|c|c|c|c|c|c|}
\hline \multirow{2}{*}{ Record number. } & \multirow{2}{*}{$\begin{array}{l}\text { Date } \\
\text { ripe. }\end{array}$} & \multirow{2}{*}{$\begin{array}{c}\text { Yield } \\
\text { (grams). }\end{array}$} & \multirow{2}{*}{$\begin{array}{l}\text { Percent- } \\
\text { age of } \\
\text { protein } \\
\text { nitrogen. }\end{array}$} & \multirow{2}{*}{$\begin{array}{l}\text { Weight } \\
\text { of aver- } \\
\text { age ker- } \\
\text { nel } \\
\text { (gram). }\end{array}$} & \multicolumn{2}{|c|}{$\begin{array}{l}\text { Protein nitrogen } \\
\text { (gram) in- }\end{array}$} \\
\hline & & & & & Kernels. & $\begin{array}{l}\text { Average } \\
\text { kernel. }\end{array}$ \\
\hline $2130 \pi \ldots \ldots \ldots$ & July 24 & 2.5691 & 3.04 & $0.01790^{\circ}$ & 0.07810 & 0.0005461 \\
\hline $21705 \ldots$ & July 23 & 1.5420 & 2.45 & $.02 i 59$ & .03778 & .0006514 \\
\hline $21708 \ldots \ldots$ & ....do... & 9.2850 & 2. 33 & .02381 & .21634 & .0005547 \\
\hline $21709 \ldots$ & ....do... & 7.7296 & 2.47 & $.021+1$ & .19092 & .0005289 \\
\hline $22206 \ldots \ldots$ & ... do... & 2.5712 & 3.22 & .01720 & .08086 & .0005538 \\
\hline 22208 & ....do... & 1.9090 & 3.18 & .01619 & .06071 & .0005144 \\
\hline $26905 \ldots$ & July 24 & 6.4102 & 2.76 & .01966 & .17692 & .0005427 \\
\hline 26908 .. & ....do do... & 3.9797 & 2.96 & .02073 & $\because 11780$ & .0006135 \\
\hline $27507 \ldots$ & July 23 & 1.3746 & 3.08 & .01833 & .04234 & .0005646 \\
\hline $27509 \ldots$ & ... do... & 5.3615 & 2.90 & .02206 & .15549 & .0006399 \\
\hline $28805 \ldots$ & ....do... & 2.1851 & 2.91 & .02512 & .04359 & .0007309 \\
\hline 28806 . & do... & 14.4630 & 3.02 & .02111 & .43679 & .0006376 \\
\hline 33106 . & do... & .3089 & 2.94 & .01716 & .00908 & .0005045 \\
\hline 33107 . & do... & 6.1026 & 2.35 & .01919 & .14341 & .0004510 \\
\hline 33405 . & do... & 8.1268 & 2.03 & .01930 & .16498 & .0003919 \\
\hline 34205 . & do... & 9.1498 & 2.73 & .01972 & .24979 & .0005383 \\
\hline $34207 \ldots \ldots$ & ....do... & 13.5556 & 2.84 & .02219 & .38505 & .0006273 \\
\hline $38506 \ldots \ldots$ & July 24 & 1. 6799 & 2.89 & .01975 & .04855 & .0005712 \\
\hline $38605 \ldots \ldots$ & July 23 & 1. 2124 & 5.85 & .01987 & .07093 & .0011627 \\
\hline $40205 \ldots \ldots$ & ....do... & 3.6302 & 4.69 & .01871 & .17026 & .0008776 \\
\hline $40305 \ldots \ldots \ldots$ & ....do.... & 3.6003 & 3.11 & .02011 & .11197 & .0006255 \\
\hline $42905 \ldots . .$. & ....do... & 1.2499 & 3.17 & .01866 & .03650 & .0005447 \\
\hline $44505 \ldots \ldots$ & ....do... & 5.9990 & 2.94 & .01764 & .17637 & .0005187 \\
\hline $44606 \ldots$ & $\ldots$ do .... & 2.5235 & 2.90 & .02035 & .07318 & .0005902 \\
\hline 45606 & $\ldots$ do.... & 4.0358 & 1.91 & .01834 & .07708 & .0003504 \\
\hline 45705. & ....do... & .7532 & 4. 18 & .01712 & .03148 & .0007155 \\
\hline $45805 \ldots$ & ....do... & 1.5298 & 1.84 & .01234 & .02815 & .0002700 \\
\hline $46107 \ldots$ & ...do... & 8.3935 & 2.54 & .01756 & .21319 & .0004460 \\
\hline $50705 \ldots$ & ...do... & .5958 & 3.54 & .01986 & .02109 & .0007032 \\
\hline $50706 \ldots$ & ... do... & .4701 & 2.80 & .01343 & .01316 & .0003761 \\
\hline $50905 \ldots$ & ...do... & 2.3982 & 3. 30 & .01085 & .07914 & .0003581 \\
\hline $55205 \ldots \ldots \ldots$ & July 24 & .6893 & 3. 10 & .01723 & .02137 & .0005342 \\
\hline $57805 \ldots . . .$. & .... do... & 4. 8988 & 2.87 & .01814 & .14060 & .0005207 \\
\hline $57905 \ldots \ldots \ldots$ & $\ldots$ do .... & 2.4731 & 3.18 & .01118 & .07859 & .0003556 \\
\hline $58505 \ldots . .$. & July 23 & 7.4516 & 2.95 & .02730 & .21982 & .0008052 \\
\hline $58705 \ldots \ldots$ & ....do... & $2.5+36$ & 3.01 & .01082 & $.0765 b^{\circ}$ & .0003258 \\
\hline $60605 \ldots$ & ....do... & .5952 & 1.87 & .01701 & .01113 & .0003180 \\
\hline 62805 & ....do... & 1.3451 & 3.25 & .01212 & .04272 & .0003938 \\
\hline 74606. & .... do .... & 9.6451 & 2.30 & .02079 & .22184 & .0004781 \\
\hline $74607 \ldots$ & $\ldots$...do... & 8.3406 & 2.56 & .01699 & .21352 & .0004349 \\
\hline $91305 \ldots$ & July 24 & 3.0940 & 3.21 & .02242 & .09932 & .0007197 \\
\hline $92505 \ldots$ & $\ldots$ do... & 2.6615 & 3.00 & .01706 & .07985 & .0005118 \\
\hline Average. . & July 23.2 & 4.9015 & 2.93 & .01878 & .13654 & .0005544 \\
\hline
\end{tabular}

DATES RIPE: JULY 27, 1903, OR LATER.

\begin{tabular}{|c|c|c|c|c|c|c|}
\hline $17307 \ldots \ldots \ldots$ & July 27 & 3. 1454 & 3. 46 & 0.02279 & 0. 10883 & 0.0007886 \\
\hline $17405 \ldots \ldots \ldots$ & ....do... & 15. 6996 & 2. 13 & .02127 & .33441 & .0004531 \\
\hline $17506 \ldots \ldots \ldots$ & ....do... & 2. 2881 & 3. 52 & .02460 & .05044 & .0008660 \\
\hline $17507 \ldots \ldots \ldots$ & ....do... & .7720 & 3. 80 & .01795 & .02934 & .0006822 \\
\hline $18905 . . . \ldots \ldots$. & .... do... & 1. 4864 & 3.81 & .01443 & .05663 & .0005498 \\
\hline $20709 \ldots \ldots \ldots$ & ....do... & 5. 3229 & 3.05 & .02063 & .16235 & .0006292 \\
\hline $21205 \ldots \ldots \ldots$ & ....do... & 2. 3642 & 3.16 & .01922 & .07471 & .0006074 \\
\hline $21206 \ldots \ldots \ldots$ & ....do... & 2.8564 & 5.23 & .01917 & .14939 & .0010026 \\
\hline $21207 \ldots \ldots \ldots$ & .... do... & 2. 3066 & 2.96 & .01955 & .06804 & .0005766 \\
\hline $21208 \ldots$ & ... do... & 5.1594 & 3.24 & .01798 & .16712 & .0005824 \\
\hline $21209 \ldots \ldots \ldots$ & do... & 1. 4484 & 3.61 & .01627 & .05228 & .0005875 \\
\hline $21210 \ldots \ldots \ldots \ldots$ & .... do... & 3.9143 & 5.0 .3 & .01577 & .19689 & .0007934 \\
\hline $21212 \ldots \ldots \ldots$ & ....do... & 1. 7216 & 2. 16 & .02049 & .03718 & .0004427 \\
\hline $21305 \ldots$ & ...do... & 6.2514 & 2.67 & .020037 & .16691 & .0005350 \\
\hline $22207 \ldots$ & ....do... & 3.2787 & 2.77 & .01940 & $.090 \times 2$ & .0005374 \\
\hline $25205 \ldots$ & .... do... & 10. 7836 & 2. $\pi 1$ & .02066 & .28560 & .0005599 \\
\hline $25206 \ldots$ & ....do... & 4. 6754 & 2. 76 & .02251 & .12904 & .0006295 \\
\hline $26106 \ldots$ & ... do... & 2.0737 & 2.63 & .02304 & .05454 & .0006060 \\
\hline $26107 \ldots$ & ....do... & 2. 0390 & 3.92 & .01416 & .07993 & .0005551 \\
\hline $26805 \ldots$ & .... do ... & 4. 9456 & 2. 81 & .02248 & .13897 & .0006317 \\
\hline $28206 \ldots$ & .... do... & 4. 3698 & 3.07 & .01996 & .13415 & .0006126 \\
\hline $32206 \ldots$ & do... & 10. 4036 & 1.81 & .02052 & $.18 \times 31$ & .0003714 \\
\hline $32207 \ldots$. & do... & 1. 2573 & 3.48 & .01822 & .04375 & .0006341 \\
\hline $32605 \ldots$. & do.. & 5. 2268 & 1.20 & .02323 & .06272 & .0002788 \\
\hline
\end{tabular}


TABLE 31. - Yield and nitrogen content of grain, tabulated according to length of growing period-Continued.

DATES RIPE: JULY 27, 1903, OR LATER-Continued.

\begin{tabular}{|c|c|c|c|c|c|c|}
\hline \multirow{2}{*}{ Record number. } & \multirow{2}{*}{$\begin{array}{l}\text { Date } \\
\text { ripe. }\end{array}$} & \multirow{2}{*}{$\begin{array}{c}\text { Yield } \\
\text { (grams). }\end{array}$} & \multirow{2}{*}{$\begin{array}{c}\text { Percent- } \\
\text { age of } \\
\text { proteid } \\
\text { nitrogen. }\end{array}$} & \multirow{2}{*}{$\begin{array}{l}\text { Weight } \\
\text { of aver- } \\
\text { age ker- } \\
\text { nel } \\
\text { (gram). }\end{array}$} & \multicolumn{2}{|c|}{$\begin{array}{l}\text { Proteid nitrogen } \\
\quad(\text { gram }) \text { in- }\end{array}$} \\
\hline & & & & & Kernels. & $\begin{array}{l}\text { Average } \\
\text { kernel. }\end{array}$ \\
\hline 32608 .. & July 27 & 1. 0183 & 3.78 & 0.01851 & 0.03849 & 0.0006998 \\
\hline 33305 .. & ....do... & 3. 1346 & 3.41 & .02090 & .10689 & .0007126 \\
\hline 33407 & ....do... & 7. 0889 & 1.62 & .02271 & .11223 & .0003679 \\
\hline $33408 \ldots .$. & .... do ... & 1. 1132 & 1. 39 & .01446 & .01547 & .0002009 \\
\hline $33605 \ldots \ldots$ & ... do ... & 7.0596 & 2. 39 & .02345 & .16872 & .0005605 \\
\hline $33606 \ldots \ldots$ & ... do... & 8. 1890 & 2. 21 & .02144 & .18098 & .0004738 \\
\hline $33607 \ldots$ & ....do... & 2.8903 & 3.22 & .02125 & .09307 & .0006843 \\
\hline $34405 \ldots .$. & .... do... & 4. 1281 & 4. 33 & .01994 & .17875 & .0008635 \\
\hline $34606 \ldots \ldots$ & .... do... & 6. 1962 & 3.12 & .02213 & .19332 & .0006904 \\
\hline $36905 \ldots$ & ....do... & 5.0200 & 3.88 & .01880 & .19478 & .0007295 \\
\hline $37305 \ldots \ldots$ & .... do... & 6. 1394 & 2.96 & .01987 & .18173 & .0005881 \\
\hline $37705 \ldots \ldots \ldots$ & .... do... & 8.0905 & 2.64 & .01972 & .23998 & .0005327 \\
\hline $37706 \ldots \ldots \ldots$ & .... do ... & 1. 2069 & 2. 34 & .02155 & .02824 & .0005053 \\
\hline $37707 \ldots \ldots$ & .... do... & 3.3004 & 2.93 & .01710 & .09670 & .0005010 \\
\hline $37905 \ldots \ldots$. & Aug. 4 & .9452 & 2.53 & .02555 & .02391 & .0006433 \\
\hline $38005 \ldots .$. & July 27 & 2.5134 & 2.84 & $.01 \varepsilon 08$ & .07138 & .0005135 \\
\hline $38505 \ldots \ldots$ & $\ldots$ do ... & 12. 1088 & 3,61 & .02252 & .43713 & .0007764 \\
\hline $39205 \ldots$. & ... do ... & 21. 5399 & 2.11 & .02089 & .45435 & .0004407 \\
\hline $39405 \ldots \ldots$ & $\ldots$ do ... & 9.3541 & 2.88 & .02093 & .21399 & .0006027 \\
\hline $39506 \ldots . .$. & Aug. 4 & 1.9218 & 2. 93 & .02869 & 05631 & .0008404 \\
\hline $39507 \ldots \ldots$ & July 27 & 1. 8862 & 3.02 & .01699 & .05696 & .0005132 \\
\hline $39606 \ldots$ & .... do ... & 4. 6383 & 2.37 & .01341 & .10967 & .0003177 \\
\hline $40505 \ldots$ & ....do... & 4. 1546 & 2. 82 & .02444 & .11716 & .0006892 \\
\hline $42205 \ldots \ldots$ & .... do. & 1. 8494 & 3.63 & .01967 & .06713 & .0007142 \\
\hline $42405 \ldots$. & ....do. & 1. 4892 & 3.07 & .02251 & .04572 & .0006927 \\
\hline $43405 \ldots$. & .... do... & 2.8000 & 2.92 & .02258 & 08176 & .0006594 \\
\hline $43505 \ldots \ldots$ & Aug. 4 & 1. 4464 & 4. 13 & .01555 & .05974 & .0006423 \\
\hline $44605 \ldots . . . .$. & July 27 & 1. 1271 & 2.86 & .02049 & .03223 & .0005861 \\
\hline $46105 \ldots . . . .$. & ....do... & 4. 6146 & 3.00 & .01775 & .13843 & .0005324 \\
\hline $46106 \ldots . . .6$ & ... do ... & 1.6103 & 2.54 & .01964 & .04090 & .0004988 \\
\hline $48705 \ldots . . . .$. & ... do ... & 4. 3615 & 3. 13 & .01652 & 13652 & .0005171 \\
\hline $49505 \ldots$ & ....do... & 1. 2716 & 3.24 & .01898 & .04120 & .0006149 \\
\hline $49805 \ldots$ & ... do ... & .6760 & 3. 62 & .02939 & .02436 & .0010640 \\
\hline $50906 \ldots$ & ... do ... & 1. 7280 & 3.57 & .01516 & .06169 & .0005411 \\
\hline $55508 \ldots \ldots$ & .... do... & 3.7407 & 3. 11 & .01732 & .11636 & .0005386 \\
\hline $58806 \ldots$ & ... do... & 1.9469 & 1.88 & .02049 & .03660 & .0003853 \\
\hline $58905 \ldots$ & ....do... & 2. 3031 & 2. 43 & .01355 & .05596 & .0003292 \\
\hline 59605. & ....do... & 7. 1828 & 2. 12 & .01880 & .15228 & .0003986 \\
\hline 63506 . & ....do... & 2.3986 & 2. 44 & .01568 & .05853 & .0003825 \\
\hline 66005. & ....do... & 7. 6690 & 2.63 & .02073 & .20170 & .0005451 \\
\hline 69506 . & .... do.. & 13. 5696 & 2.50 & .02047 & .33923 & .0005117 \\
\hline 69805 . & ....do... & 2.4420 & 5. 82 & .02220 & .14213 & .0012921 \\
\hline 69806 . & ....do... & 12.0136 & 1.66 & .02153 & .19943 & .0003574 \\
\hline 72405. & .... do $\ldots$ & 8. 4415 & 3.36 & .03963 & .28363 & .0013316 \\
\hline 72406 . & ...do do ... & 8. 2929 & 2.95 & 01929 & .24464 & .0005689 \\
\hline 72905 . & ....do... & 2. 6462 & 2. 48 & .01585 & .06563 & .0003930 \\
\hline 73307. & .... do ... & .5572 & 2.39 & .02229 & .01332 & .0005327 \\
\hline 73308 . & ....do... & 14. 2986 & 2.92 & .02291 & .41752 & .0006539 \\
\hline 74305 . & ...do... & 4. 4222 & 1.98 & .02047 & .08756 & .0004054 \\
\hline 74506 . & ... do... & .4096 & 2. 73 & .01781 & .01118 & .0004862 \\
\hline $74508 \ldots$. & .... do... & .8172 & 2. 60 & .01434 & .02125 & .0003728 \\
\hline 76205 . & ...do... & 8.4407 & 2.35 & .01695 & .19836 & .0003982 \\
\hline 80305 & ....do... & 15. 7835 & 1.81 & .02165 & 28569 & .0003919 \\
\hline 81405. & .... do... & 4. 5737 & 2.62 & .01862 & .11710 & .0004879 \\
\hline 81406 . & ...do... & 1. 2391 & 3.31 & .01721 & .04101 & .0005697 \\
\hline 84405 . & .... do... & 8.7448 & 2. 48 & .02043 & .21687 & .0005067 \\
\hline 85205 . & ....do... & 3.4766 & 2.60 & .01625 & .09039 & .0004224 \\
\hline 86105. & .... do... & 3.0282 & 2.56 & .01495 & .07964 & .0003923 \\
\hline 86106. & $\ldots$ do... & 7.6241 & 2.63 & .01749 & .20052 & .0004599 \\
\hline Average.. & July 27.2 & $4.66: 6$ & 2.94 & .01992 & .12854 & .0005800 \\
\hline
\end{tabular}


TABLE 32.-Summary of yield and nitrogen content of grain, tabulated according to length of growing period.

\begin{tabular}{|c|c|c|c|c|c|c|c|}
\hline \multirow{2}{*}{$\begin{array}{l}\text { Plants grouped according to } \\
\text { date ripe. }\end{array}$} & \multirow{2}{*}{$\begin{array}{l}\text { Num- } \\
\text { ber of } \\
\text { anal- } \\
\text { yses. }\end{array}$} & \multirow{2}{*}{$\begin{array}{l}\text { Average } \\
\text { date ripe. }\end{array}$} & \multirow{2}{*}{$\begin{array}{c}\text { Yield } \\
\text { (grams). }\end{array}$} & \multirow{2}{*}{$\begin{array}{l}\text { Percent- } \\
\text { age of } \\
\text { proteid } \\
\text { nitrogen. }\end{array}$} & \multirow{2}{*}{$\begin{array}{c}\text { Weight } \\
\text { of aver- } \\
\text { age } \\
\text { kernel } \\
\text { (gram). }\end{array}$} & \multicolumn{2}{|c|}{$\begin{array}{l}\text { Proteid nitrogen } \\
\text { (gram) in- }\end{array}$} \\
\hline & & & & & & Kernels. & $\begin{array}{l}\text { Average } \\
\text { kernel. }\end{array}$ \\
\hline 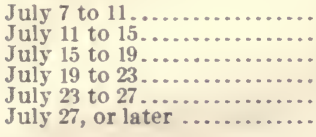 & $\begin{array}{l}49 \\
65 \\
50 \\
56 \\
52 \\
83\end{array}$ & $\begin{array}{l}\text { July } 8.9 \ldots \\
\text { July } 13 \ldots \\
\text { July } 16.2 . \\
\text { July } 20.1 \ldots \\
\text { July } 23.2 . \\
\text { July } 27.2 . .\end{array}$ & $\begin{array}{l}9.9067 \\
7.6611 \\
5.1354 \\
6.5399 \\
4.9015 \\
4.6636\end{array}$ & $\begin{array}{l}2.69 \\
2.81 \\
2.87 \\
2.93 \\
2.93 \\
2.94\end{array}$ & $\begin{array}{r}0.02024 \\
.01887 \\
.01869 \\
.01886 \\
.01878 \\
.01992\end{array}$ & $\begin{array}{r}0.26475 \\
.20820 \\
.14452 \\
.18064 \\
.13654 \\
.12854\end{array}$ & $\begin{array}{r}0.0005356 \\
.0005290 \\
.0005222 \\
.0005482 \\
.0005544 \\
.0005800\end{array}$ \\
\hline
\end{tabular}

TABLE 33.-Summary of nitrogen content, etc., tabulated according to yield per plant.

\begin{tabular}{|c|c|c|c|c|c|c|c|}
\hline \multirow{2}{*}{$\begin{array}{l}\text { Plants grouped according to } \\
\text { yield (in grams). }\end{array}$} & \multirow{2}{*}{$\begin{array}{l}\text { Num- } \\
\text { ber of } \\
\text { anal- } \\
\text { yses. }\end{array}$} & \multirow{2}{*}{$\begin{array}{l}\text { Average } \\
\text { date ripe. }\end{array}$} & \multirow{2}{*}{$\begin{array}{l}\text { Yield } \\
\text { (grams). }\end{array}$} & \multirow{2}{*}{$\begin{array}{l}\text { Percent- } \\
\text { age of } \\
\text { proteid } \\
\text { nitrogen. }\end{array}$} & \multirow{2}{*}{$\begin{array}{c}\text { Weight } \\
\text { of a ver- } \\
\text { age } \\
\text { kernel } \\
\text { (gram). }\end{array}$} & \multicolumn{2}{|c|}{$\begin{array}{l}\text { Proteid nitrogen } \\
\text { (gram) in- }\end{array}$} \\
\hline & & & & & & Kernels. & $\begin{array}{l}\text { Average } \\
\text { kernel. }\end{array}$ \\
\hline 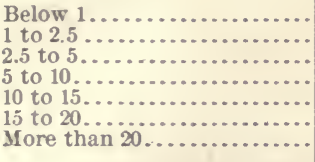 & $\begin{array}{r}31 \\
67 \\
88 \\
94 \\
52 \\
20 \\
4\end{array}$ & $\begin{array}{l}\text { July } 20.2 . \\
\text { July } 21.9 . \\
\text { July } 20 \ldots \\
\text { July } 18.3 . \\
\text { July } 15.1 . \\
\text { July } 15.1 . \\
\text { July } 14.5 .\end{array}$ & $\begin{array}{r}0.6049 \\
1.7673 \\
3.5683 \\
7.6706 \\
12.2573 \\
17.1908 \\
23.7186\end{array}$ & $\begin{array}{l}2.91 \\
3.09 \\
3.03 \\
2.68 \\
2.71 \\
2.54 \\
2.55\end{array}$ & $\begin{array}{r}0.01683 \\
.01852 \\
.01796 \\
.01997 \\
.02168 \\
.02103 \\
.02159\end{array}$ & $\begin{array}{r}0.01731 \\
.05456 \\
.10794 \\
.20270 \\
.33433 \\
.43921 \\
.60401\end{array}$ & $\begin{array}{r}0.0004916 \\
.0005730 \\
.0005445 \\
.0005351 \\
.0005774 \\
.0005382 \\
.0005450\end{array}$ \\
\hline
\end{tabular}

TABLE 34.-Summary of yield, etc., tabulated according to nitrogen content.

\begin{tabular}{|c|c|c|c|c|c|c|c|}
\hline \multirow{2}{*}{$\begin{array}{l}\text { Plants grouped according to } \\
\text { percentage of nitrogen. }\end{array}$} & \multirow{2}{*}{$\begin{array}{l}\text { Num- } \\
\text { ber of } \\
\text { anal- } \\
\text { yses. }\end{array}$} & \multirow{2}{*}{$\begin{array}{l}\text { Average } \\
\text { date ripe. }\end{array}$} & \multirow{2}{*}{$\begin{array}{c}\text { Yield } \\
\text { (grams). }\end{array}$} & \multirow{2}{*}{$\begin{array}{l}\text { Percent- } \\
\text { age of } \\
\text { proteid } \\
\text { nitrogen. }\end{array}$} & \multirow{2}{*}{$\begin{array}{c}\text { Weight } \\
\text { of aver- } \\
\text { age } \\
\text { kernel } \\
\text { (gram). }\end{array}$} & \multicolumn{2}{|c|}{$\begin{array}{l}\text { Proteid nitrogen } \\
\text { (gram) in- }\end{array}$} \\
\hline & & & & & & Kernels. & $\begin{array}{l}\text { Average } \\
\text { kernel. }\end{array}$ \\
\hline 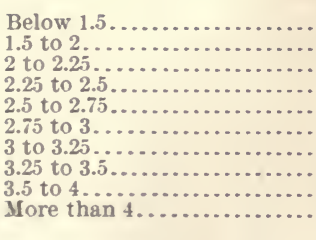 & $\begin{array}{r}4 \\
25 \\
18 \\
47 \\
82 \\
67 \\
47 \\
20 \\
23 \\
25\end{array}$ & $\begin{array}{l}\text { July } 22.5 . \\
\text { July } 18.5 . \\
\text { July } 19.8 . \\
\text { July } 17.3 . \\
\text { July 16.3.. } \\
\text { July 19.6.. } \\
\text { July } 21.2 . \\
\text { July } 20.7 . \\
\text { July 21.5. } \\
\text { July 19.5.. }\end{array}$ & $\begin{array}{l}5.8099 \\
2.7423 \\
8.9542 \\
7.3389 \\
8.0817 \\
5.9093 \\
4.4497 \\
4.6756 \\
3.6486 \\
4.5431\end{array}$ & $\begin{array}{l}1.35 \\
1.80 \\
2.12 \\
2.39 \\
2.63 \\
2.85 \\
3.11 \\
3.37 \\
3.68 \\
4.72\end{array}$ & $\begin{array}{r}0.01709 \\
.02124 \\
.02030 \\
.02000 \\
.01938 \\
.01910 \\
.01824 \\
.01870 \\
.01852 \\
.01819\end{array}$ & $\begin{array}{r}0.07290 \\
.11620 \\
.19070 \\
.18478 \\
.21280 \\
.16609 \\
.13847 \\
.15189 \\
.13513 \\
.21239\end{array}$ & $\begin{array}{r}0.0002266 \\
.0003867 \\
.0004325 \\
.0004773 \\
.0005102 \\
.0005454 \\
.0005667 \\
.0006213 \\
.0006807 \\
.0008639\end{array}$ \\
\hline
\end{tabular}

\section{RELATION OF SIZE OF HEAD TO YIELD, HEIGHT, AND TILLERING OF PLANT.}

The size of the head has always been considered to be closely connected with the productiveness of wheat. The well-known work of Hallet in increasing the yielding qualities of wheat is perhaps the best example of wheat improvement by the selection of plants having large heads. Whether large heads or a large number of mediumsized heads on a plant are more desirable is still a question.

Table 35 gives the yields, etc., of between 300 and 400 plants, tabulated according to the number of kernels on the head. Table 36 is a summary of these, while Tables 37 and 38 consist of the same data tabulated according to the yield per plant and yield per head, respectively. 
It will be seen from Table 36 that the heads of slightly more than medium size produced the largest yields of grain; that the weight of the average kernel did not increase with the size of the head, nor did it decrease except on the very largest heads; that the plants with somewhat more than average-sized heads were the tallest, and that the plants with medium-sized heads or slightly less tillered most largely.

Table 37 shows that with an increased yield per plant there is a constant increase in the height and tillering of the plant.

Table 38 indicates that the yield per head and yield per plant do not increase together, but that the largest yielding plants are those of medium yield per head. The same would seem to be true of the height and tillering of the plant. The weight of the average kernel increases quite uniformly with the yield per head.

In considering these results it must be borne in mind that these plants were grown 6 inches apart each way, and were therefore not under the conditions that would obtain in a thickly drilled or broadcasted field, where lack of ability to tiller would be compensated for by the larger number of plants. However, the variety of wheat yielding best in Nebraska is one having only a medium-sized or even small head, as compared with most wheats, but it is a strongtillering variety.

TABLE 35.-Relation of size of head to yield, height, and tillering of plant.

SIZE OF HEAD, BELOW 16 KERNELS.

\begin{tabular}{|c|c|c|c|c|c|c|}
\hline $\begin{array}{c}\text { Record num- } \\
\text { ber. }\end{array}$ & $\begin{array}{l}\text { Size of } \\
\text { head. }\end{array}$ & $\begin{array}{l}\text { Yield per } \\
\text { plant } \\
\text { (grams). }\end{array}$ & $\begin{array}{l}\text { Yield per } \\
\text { head } \\
\text { (grams). }\end{array}$ & $\begin{array}{l}\text { Weight of } \\
\text { average } \\
\text { kernel } \\
\text { (grams). }\end{array}$ & $\begin{array}{l}\text { Height } \\
\text { (cm.). }\end{array}$ & Tillering. \\
\hline 17308. & 15.2 & 1.2275 & 0.3069 & 0.02012 & 59 & 5 \\
\hline $17406 .$. & 15.5 & 2.0907 & .2613 & .01686 & 65 & 11 \\
\hline $18805 \ldots$ & 15.2 & 2.1462 & .2385 & .01567 & 65 & 18 \\
\hline 20708 . & 13.6 & 2.4690 & .2743 & .02024 & 60 & 11 \\
\hline 21211 . & 10.0 & .2806 & .2806 & .02806 & 45 & 2 \\
\hline 22209 . . & 15.5 & .4336 & .2168 & .01399 & 70 & 6 \\
\hline 26805 & 15.7 & 4.9456 & .3533 & .02248 & 68 & 26 \\
\hline 32207 . & 13.8 & 1.2573 & .2515 & .01822 & 47 & 5 \\
\hline $37905 \ldots$ & 12.3 & .9452 & .3151 & .02555 & 52 & 3 \\
\hline $39506 \ldots$ & 11.2 & 1.9218 & .3203 & .02869 & 48 & 6 \\
\hline 42206 .. & 12.5 & .3161 & .1580 & .01264 & 63 & 5 \\
\hline 44607. & 12.6 & 2.5235 & .2281 & .02035 & 52 & 12 \\
\hline 48408 . & 13.5 & .3485 & .1742 & .01291 & 45 & 3 \\
\hline 49905 . & 11.5 & .6760 & .3380 & .02939 & 49 & 2 \\
\hline 50705. & 15.0 & .5958 & .2979 & .01986 & 40 & 3 \\
\hline 73307. & 12.5 & .5572 & .2786 & .02229 & 46 & 4 \\
\hline 74506 . & 12.5 & .4096 & .2048 & .01781 & 68 & 2 \\
\hline 94105 . & 11.0 & .5595 & .2797 & .02543 & 51 & 1 \\
\hline A verage .. & 13.3 & 1.3169 & .2654 & .02059 & 55.2 & 6.9 \\
\hline
\end{tabular}

SIZE OF HEAD, 16 TO 20 KERNELS.

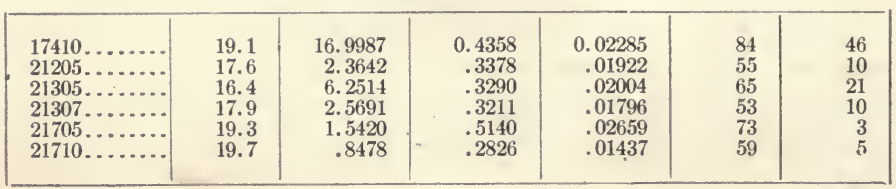


TABLE 35.-Relation of size of head to yield, height, and tillering of plant-Continued.

SIZE OF HEAD, 16 TO 20 KERNELS-Continued.

\begin{tabular}{|c|c|c|c|c|c|c|}
\hline $\begin{array}{l}\text { Record num- } \\
\text { ler. }\end{array}$ & $\begin{array}{l}\text { Size of } \\
\text { head. }\end{array}$ & $\begin{array}{c}\text { Yield per } \\
\text { plant } \\
\text { (grams). }\end{array}$ & $\begin{array}{l}\text { Yield per } \\
\text { head } \\
\text { (grams). }\end{array}$ & $\begin{array}{l}\text { Weight of } \\
\text { average } \\
\text { kernel } \\
\text { (grams). }\end{array}$ & $\begin{array}{l}\text { Height } \\
\text { (cm.). }\end{array}$ & Tillering. \\
\hline $21807 \ldots \ldots$ & 18.8 & 9.4172 & 0.4709 & 0.02498 & 77 & 25 \\
\hline $22207 \ldots \ldots$ & 18.8 & 3.2787 & .3643 & .01940 & 65 & 16 \\
\hline $22208 \ldots$ & 16.8 & 1.9090 & .2727 & .01619 & 57 & 8 \\
\hline $26906 \ldots \ldots$ & 19.0 & 4. 2376 & .3531 & .01859 & 70 & 16 \\
\hline $26909 \ldots \ldots$ & 18.0 & 2.9999 & .3000 & .01667 & 50 & 10 \\
\hline $28206 \ldots \ldots$ & 19.9 & 4. 3698 & .3972 & .01996 & 80 & 26 \\
\hline $33106 \ldots \ldots$ & 18.0 & .3089 & .3089 & .01716 & 43 & 2 \\
\hline $37706 \ldots \ldots$ & 18.7 & 1. 2069 & .4023 & .02155 & 42 & 4 \\
\hline $37906 \ldots \ldots \ldots$ & 19.0 & .2063 & .2063 & .01086 & 50 & 2 \\
\hline $38005 \ldots \ldots$ & 19.8 & 2.5134 & .3591 & .01808 & 53 & 7 \\
\hline $38607 \ldots \ldots$ & 19.0 & .3037 & .3037 & .01598 & 56 & 2 \\
\hline $38608 \ldots \ldots \ldots$ & 17.6 & 3.0228 & .3359 & .01913 & 60 & 11 \\
\hline $38609 \ldots \ldots$ & 19.5 & 6.7665 & .4511 & .02309 & 65 & 6 \\
\hline $42205 \ldots \ldots$. & 18.8 & 1.8194 & .1699 & .01967 & 68 & 6 \\
\hline $44605 \ldots \ldots$ & 18.3 & 1.1271 & .3757 & .02049 & 53 & 3 \\
\hline $44606 \ldots \ldots$ & 17.7 & 2.5235 & .3605 & .02035 & 52 & 8 \\
\hline $48105 \ldots \ldots$ & 19.0 & .9701 & .2425 & .01276 & 55 & 5 \\
\hline $50706 \ldots$ & 17.5 & .4701 & .2350 & .01343 & 38 & 2 \\
\hline $55905 \ldots \ldots$ & 18.4 & 5. 7948 & .3219 & .01751 & 75 & 34 \\
\hline $55906 \ldots \ldots$ & 19.2 & 7.9968 & .3076 & .01603 & 85 & 40 \\
\hline $56105 \ldots \ldots$ & 17.7 & 5. 7431 & .3023 & .01709 & 70 & 35 \\
\hline $56207 \ldots \ldots$ & 17.7 & 10.9073 & .4195 & .02361 & 84 & 42 \\
\hline $57307 \ldots \ldots$ & 16.3 & 4.7117 & .2945 & 01801 & 67 & 17 \\
\hline $69705 \ldots$ & 17.4 & 3.7810 & .2701 & .01550 & 88 & 28 \\
\hline $74508 \ldots \ldots$ & 19.0 & .8172 & .2724 & .01434 & 50 & 4 \\
\hline $81708 \ldots$ & 19.1 & 7.3993 & .4933 & .02578 & 86 & 20 \\
\hline $88608 \ldots \ldots$ & 18.5 & 1.5355 & .3839 & .02075 & 69 & 4 \\
\hline $92207 \ldots \ldots$ & 19.0 & 3.6926 & .3357 & .01767 & 73 & 15 \\
\hline $92505 \ldots \ldots$ & 17.3 & 2. 6615 & .2957 & .01706 & 68 & 12 \\
\hline $95510 \ldots \ldots$ & 19.9 & 2.8356 & .3544 & .01783 & 70 & 8 \\
\hline A verage .. & 18.4 & 3.7758 & .3383 & .01862 & 64.1 & 13.7 \\
\hline
\end{tabular}

SIZE OF IIEAD, 20 TO 24 KERNELS.

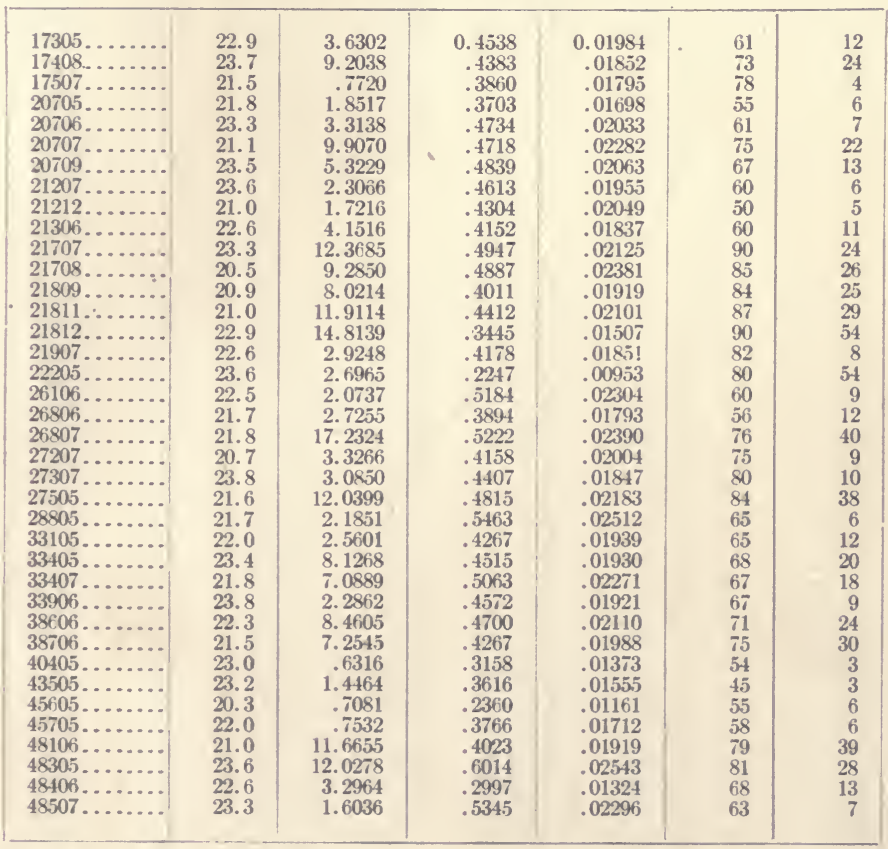


TABLE 35.-Relation of size of head to yield, height, and tillering of plant-Continued.

SIZE OF HEAD, 20 TO 24 KERNELS-Continued.

\begin{tabular}{|c|c|c|c|c|c|c|}
\hline $\begin{array}{l}\text { Record num- } \\
\text { ber. }\end{array}$ & $\begin{array}{l}\text { Size of } \\
\text { head. }\end{array}$ & $\begin{array}{l}\text { Yield per } \\
\text { plant } \\
\text { (grams). }\end{array}$ & $\begin{array}{l}\text { Yield per } \\
\text { head } \\
\text { (grams). }\end{array}$ & $\begin{array}{c}\text { Weight of } \\
\text { average } \\
\text { kernel } \\
\text { (grams). }\end{array}$ & $\begin{array}{l}\text { Height } \\
\text { (cm.). }\end{array}$ & Tillering. \\
\hline $48806 \ldots \ldots \ldots$ & 21.0 & 9.8346 & 0.3782 & 0.01798 & 78 & 12 \\
\hline $55205 \ldots \ldots$ & 20.0 & .6893 & .3446 & .01723 & 56 & 6 \\
\hline $55606 \ldots . .$. & 22.9 & 11.0930 & .5042 & .02205 & 92 & 24 \\
\hline $55907 \ldots \ldots$ & 21.4 & 19.3966 & .5542 & .02590 & 95 & 42 \\
\hline $55908 \ldots \ldots$ & 23.4 & 12.2210 & .5092 & .02175 & 95 & 40 \\
\hline $55909 \ldots \ldots$ & 21.5 & 9.2120 & .6580 & .03050 & 85 & 31 \\
\hline $56205 \ldots \ldots$ & 23.8 & 6.5232 & .4659 & .01959 & 82 & 29 \\
\hline $56206 \ldots \ldots$ & 20.4 & 9.3093 & .3724 & .01829 & 86 & 42 \\
\hline $56208 \ldots$ & 22.5 & 13.5720 & .5429 & .02356 & 88 & 51 \\
\hline $56209 \ldots \ldots$ & 21.1 & 15.8086 & .3513 & .01664 & 90 & 67 \\
\hline $57005 \ldots \ldots$ & 22.0 & 1.5364 & .3841 & .01746 & 73 & 7 \\
\hline $57105 \ldots \ldots$ & 23.9 & 3.7263 & .2192 & .00916 & 85 & 40 \\
\hline $57305 \ldots \ldots$ & 22.8 & 8.5777 & .3899 & .01666 & 78 & 30 \\
\hline $57306 \ldots \ldots$ & 21.7 & 7.9772 & .3989 & .01838 & 80 & 23 \\
\hline $57308 \ldots \ldots$ & 21.4 & 9.8378 & .3644 & .01705 & 80 & 40 \\
\hline $57506 \ldots$ & 22.5 & 2.7616 & .3452 & .01534 & 72 & 18 \\
\hline $57507 \ldots$ & 23.9 & 6.9861 & .4657 & .01946 & 78 & 26 \\
\hline $57508 \ldots \ldots$ & 22.3 & 12.0728 & .7102 & .03177 & 85 & 22 \\
\hline $63105 \ldots \ldots$ & 22.5 & 1.5452 & .3883 & .01717 & 68 & 8 \\
\hline $63106 \ldots$ & 23.6 & 3.3006 & .4715 & .02001 & 77 & 9 \\
\hline $63107 \ldots \ldots \ldots$ & $\begin{array}{l}20.0 \\
21.9\end{array}$ & 9.3120 & .4901 & .02233 & 80 & 25 \\
\hline $72605 \ldots \ldots$ & 21.7 & 1.1166 & .3722 & .01718 & 52 & 3 \\
\hline $72705 \ldots \ldots$ & 21.9 & 9.1522 & .5384 & .02191 & 68 & 20 \\
\hline $74305 \ldots \ldots$ & 21.6 & 4.4222 & .4422 & .02047 & 60 & 11 \\
\hline $74507 \ldots \ldots$ & 20.5 & 9.2130 & .3839 & .01869 & 70 & 27 \\
\hline $74605 \ldots \ldots$ & 21.0 & 7.1181 & .3746 & .01784 & 69 & 27 \\
\hline $74606 \ldots \ldots$ & 23.2 & 9.6451 & .4822 & .02079 & 75 & 24 \\
\hline $76205 \ldots \ldots$ & 21.7 & 8.4407 & .3670 & .01695 & 70 & 26 \\
\hline $81405 \ldots \ldots$ & 21.8 & 4.5737 & .4158 & .01862 & 70 & 11 \\
\hline $81705 \ldots \ldots$ & 21.1 & 9.7922 & .4451 & .02106 & 82 & 27 \\
\hline $81706 \ldots \ldots$ & 21.2 & 15.3928 & .4527 & .02132 & 90 & 40 \\
\hline $81707 \ldots .$. & 23.8 & 18.3614 & .5564 & .02336 & 96 & 53 \\
\hline $81709 \ldots \ldots$ & 20.5 & 16.4692 & .4451 & .02175 & 90 & 45 \\
\hline $84405 \ldots . .$. & 23.8 & 8.7448 & .4858 & .02043 & 75 & 19 \\
\hline $88607 \ldots$ & 23.4 & 5. 1584 & .5158 & .02205 & 73 & 15 \\
\hline $91905 \ldots . .$. & 22.0 & 3.4436 & .3826 & .01739 & 72 & 12 \\
\hline $91906 \ldots \ldots$ & 22.2 & 3.5486 & .3943 & .01774 & 74 & 11 \\
\hline $92206 \ldots \ldots$ & 23.0 & 1.1074 & .5537 & .02407 & 66 & 3 \\
\hline $92305 \ldots \ldots \ldots$ & 22.9 & 2.3859 & .3408 & .01491 & 65 & . 11 \\
\hline $92306 \ldots \ldots$ & 23.1 & 6.0091 & .4006 & .01732 & 75 & 19 \\
\hline $92506 \ldots \ldots$ & 22.9 & 3.8709 & 3871 & .01690 & 77 & -16 \\
\hline \multirow{2}{*}{ Average. } & 22.0 & 9.6779 & .4208 & .01916 & 82 & 29 \\
\hline & 22.2 & 6.8466 & .4355 & .01953 & 73.8 & 21.4 \\
\hline
\end{tabular}

SIZE OF HEAD, 24 TO 28 KERNELS.

\begin{tabular}{|c|c|c|c|c|c|c|}
\hline $17306 \ldots \ldots \ldots$ & 24.3 & 3.9968 & 0.3997 & 0.01645 & 66 & 12 \\
\hline $17405 \ldots \ldots$ & 25.1 & 15. 6996 & .5414 & .02127 & 72 & 34 \\
\hline $17409 \ldots$ & 24.3 & 14.8957 & .4514 & .01857 & 85 & 39 \\
\hline $20710 \ldots \ldots$ & 25.5 & 17.1115 & .5032 & .01974 & 77 & 39 \\
\hline $21206 \ldots \ldots$ & 24.8 & 2.8564 & .4761 & .01917 & 62 & 6 \\
\hline $21308 \ldots \ldots$ & 25.3 & 5.8080 & .4149 & .01641 & 54 & 14 \\
\hline $21706 \ldots \ldots$ & 26.9 & 19.3318 & .6444 & .02390 & 88 & 38 \\
\hline $21709 \ldots \ldots$ & 25.8 & 7.7296 & .5521 & .02141 & 85 & 23 \\
\hline $21711 \ldots \ldots$ & 24.2 & 17.1820 & .4773 & .01968 & 85 & 51 \\
\hline $21805 \ldots \ldots \ldots$ & 24.9 & 14.2450 & .5935 & .02378 & 91 & 32 \\
\hline $21808 \ldots \ldots$ & 25.7 & 19.7446 & .4388 & .01708 & 96 & 57 \\
\hline $21810 \ldots \ldots$ & 26.0 & 1.0304 & .5152 & .01982 & 55 & 4 \\
\hline $21913 \ldots \ldots$ & 27.3 & 10.1925 & .5662 & .02072 & 84 & 27 \\
\hline $22210 \ldots \ldots \ldots$ & 27.1 & 6.0173 & .5470 & .02019 & 78 & 31 \\
\hline $26808 \ldots \ldots \ldots$ & 24.7 & 3.8811 & .4312 & .01748 & 64 & 11 \\
\hline $26905 \ldots . .$. & 25.1 & 6.4102 & .4931 & .01966 & 66 & 15 \\
\hline $26908 \ldots \ldots$ & 24.0 & 3.9797 & .4974 & .02073 & 62 & 9 \\
\hline $27205 \ldots . .$. & 26.2 & 16.4061 & .4825 & .01841 & 87 & 57 \\
\hline $27305 \ldots \ldots$ & 24.3 & 5.5666 & .5061 & .02085 & 80 & 22 \\
\hline $27506 \ldots \ldots$ & 24.7 & 10.0005 & .5556 & .02252 & 85 & 23 \\
\hline $27507 \ldots \ldots$ & 25.0 & 1.3746 & .4582 & .01833 & 50 & 4 \\
\hline $27508 \ldots \ldots$ & 27.9 & 5.5324 & .6137 & .02287 & 78 & 19 \\
\hline $32608 \ldots \ldots$ & 27.5 & 1.0183 & .5091 & .01851 & 50 & 2 \\
\hline $33107 \ldots \ldots \ldots$ & 24.5 & 6. 1026 & .4694 & .01919 & 73 & 29 \\
\hline $33305 \ldots$ & 25.0 & 3.1346 & .5224 & .02090 & 53 & 7 \\
\hline $33406 \ldots$ & 25.7 & 4.6045 & .4186 & .01627 & 72 & 16 \\
\hline $33408 \ldots \ldots$ & 25.7 & 1.1132 & .3711 & .01446 & 56 & 4 \\
\hline
\end{tabular}


TABLE 35.-Relation of size of head to yield, height, and tillering of plant-Continued.

SIZE OF IIEAD, 24 TO 28 KERNELS-Continued.

\begin{tabular}{|c|c|c|c|c|c|c|}
\hline $\begin{array}{c}\text { Record num- } \\
\text { ber. }\end{array}$ & $\begin{array}{l}\text { Size of } \\
\text { head. }\end{array}$ & $\begin{array}{c}\text { Yield per } \\
\text { plant } \\
\text { (grams). }\end{array}$ & $\begin{array}{l}\text { Yield per } \\
\text { head } \\
\text { (grams). }\end{array}$ & $\begin{array}{c}\text { Weight of } \\
\text { average } \\
\text { kernel } \\
\text { (grams). }\end{array}$ & $\begin{array}{l}\text { Height } \\
\text { (cm.). }\end{array}$ & Tillering \\
\hline 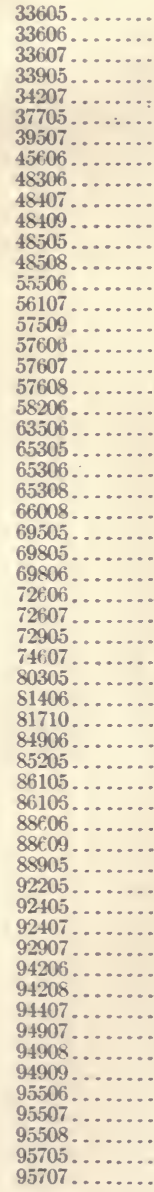 & $\begin{array}{l}27.4 \\
27.3 \\
27.2 \\
26.7 \\
26.6 \\
25.6 \\
27.8 \\
24.4 \\
26.2 \\
26.6 \\
26.2 \\
27.4 \\
27.4 \\
27.1 \\
24.9 \\
27.8 \\
26.4 \\
27.3 \\
24.3 \\
24.7 \\
25.5 \\
26.0 \\
25.9 \\
26.5 \\
24.9 \\
25.5 \\
27.5 \\
27.9 \\
27.1 \\
26.9 \\
27.8 \\
25.8 \\
25.1 \\
24.0 \\
24.7 \\
25.5 \\
26.7 \\
25.4 \\
27.2 \\
25.3 \\
24.7 \\
26.6 \\
26.5 \\
26.7 \\
26.5 \\
24.3 \\
25.1 \\
24.8 \\
26.2 \\
27.2 \\
25.0 \\
24.2 \\
25.9 \\
26.0 \\
25.5 \\
26.5 \\
26.0 \\
\\
\end{array}$ & $\begin{array}{r}7.0596 \\
8.1890 \\
2.8903 \\
11.1476 \\
13.5556 \\
8.0905 \\
1.8862 \\
4.0358 \\
2.6571 \\
11.2890 \\
6.4302 \\
1.9154 \\
11.2008 \\
17.8506 \\
14.4556 \\
10.6261 \\
3.0790 \\
16.4433 \\
8.6189 \\
1.3961 \\
2.3986 \\
1.8018 \\
9.8298 \\
11.7066 \\
3.1555 \\
4.7116 \\
2.4420 \\
12.0136 \\
9.3629 \\
3.4442 \\
2.6462 \\
8.3406 \\
15.7835 \\
1.2391 \\
9.1411 \\
7.5438 \\
3.4766 \\
3.0282 \\
7.6241 \\
9.9456 \\
9.8719 \\
5.3069 \\
5.2616 \\
3.4356 \\
.8983 \\
4.4673 \\
7.5006 \\
3.7828 \\
6.7664 \\
12.1918 \\
2.3678 \\
3.6977 \\
11.0548 \\
12.1592 \\
14.4617 \\
10.3426 \\
.7577\end{array}$ & $\begin{array}{l}0.6418 \\
.5489 \\
.5781 \\
.5867 \\
.5894 \\
.4495 \\
.4715 \\
.4484 \\
.4428 \\
.4181 \\
.5358 \\
.3831 \\
.5091 \\
.5578 \\
.3023 \\
.4830 \\
.6158 \\
.6090 \\
.4788 \\
.2327 \\
.3998 \\
.6006 \\
.4681 \\
.5321 \\
.4505 \\
.4712 \\
.6105 \\
.6007 \\
.4681 \\
.4920 \\
.4410 \\
.4390 \\
.5442 \\
.4130 \\
. .5713 \\
.5029 \\
.4386 \\
.3785 \\
.4765 \\
.5234 \\
.5196 \\
.4824 \\
.4047 \\
.4294 \\
.4491 \\
.4964 \\
.4688 \\
.2909 \\
.4229 \\
.5301 \\
.4736 \\
.2631 \\
.4806 \\
.5527 \\
.4987 \\
.4309 \\
.3788\end{array}$ & $\begin{array}{r}0.02345 \\
.02144 \\
.02125 \\
.02194 \\
.02219 \\
.01972 \\
.01699 \\
.01834 \\
.01692 \\
.01572 \\
.02048 \\
.01398 \\
.01858 \\
.02062 \\
.01658 \\
.01739 \\
.02333 \\
.02234 \\
.01968 \\
.00943 \\
.01568 \\
.02310 \\
.01807 \\
.02008 \\
.01814 \\
.01847 \\
.02220 \\
.02153 \\
.01724 \\
.01832 \\
.01585 \\
.01699 \\
.02165 \\
.01721 \\
.02308 \\
.01975 \\
.01625 \\
.01495 \\
.01749 \\
.02068 \\
.02100 \\
.01811 \\
.01525 \\
.01605 \\
.01695 \\
.02040 \\
.01866 \\
.01175 \\
.01615 \\
.01948 \\
.01894 \\
.01696 \\
.01852 \\
.02029 \\
.01954 \\
.01626 \\
.01457\end{array}$ & $\begin{array}{l}65 \\
72 \\
58 \\
77 \\
77 \\
60 \\
59 \\
59 \\
58 \\
82 \\
74 \\
70 \\
80 \\
95 \\
90 \\
84 \\
78 \\
87 \\
83 \\
75 \\
64 \\
65 \\
75 \\
77 \\
76 \\
66 \\
62 \\
75 \\
82 \\
74 \\
59 \\
76 \\
70 \\
55 \\
90 \\
65 \\
65 \\
68 \\
76 \\
85 \\
74 \\
82 \\
72 \\
78 \\
68 \\
84 \\
76 \\
71 \\
82 \\
85 \\
73 \\
72 \\
86 \\
90 \\
97 \\
80 \\
67\end{array}$ & $\begin{array}{r}14 \\
17 \\
6 \\
23 \\
22 \\
22 \\
4 \\
13 \\
7 \\
53 \\
19 \\
7 \\
36 \\
58 \\
49 \\
37 \\
8 \\
48 \\
38 \\
29 \\
7 \\
10 \\
28 \\
35 \\
8 \\
13 \\
7 \\
28 \\
26 \\
8 \\
5 \\
31 \\
33 \\
3 \\
24 \\
16 \\
11 \\
4 \\
25 \\
23 \\
26 \\
17 \\
18 \\
10 \\
2 \\
10 \\
19 \\
19 \\
23 \\
23 \\
9 \\
9 \\
25 \\
22 \\
31 \\
31 \\
4\end{array}$ \\
\hline Averagè & 25.9 & T.5207 & .4848 & .01874 & 73.8 & 21.2 \\
\hline
\end{tabular}

SIZE OF HEAD, 28 TO 32 KERNELS.

\begin{tabular}{|r|r|r|r|r|r|r|}
\hline $17505 \ldots \ldots \ldots$ & 29.0 & 0.3885 & 0.3885 & 0.01340 & 46 & 7 \\
$17506 \ldots \ldots \ldots$ & 31.0 & 2.2881 & .7627 & .02460 & 55 & 6 \\
$20805 \ldots \ldots \ldots$ & 31.7 & 14.6942 & .6679 & .02157 & 85 & 30 \\
$21208 \ldots \ldots \ldots$ & 28.7 & 5.1594 & .5159 & .01798 & 63 & 11 \\
$21209 \ldots \ldots \ldots$. & 29.7 & 1.4484 & .4828 & .01627 & 51 & 6 \\
$21210 \ldots \ldots \ldots$ & 29.6 & 3.9143 & .4893 & .01577 & 59 & 8 \\
$21805 \ldots \ldots \ldots$. & 29.3 & 20.9290 & .4983 & .01699 & 91 & 48 \\
$21905 \ldots \ldots \ldots$ & 28.2 & 14.3111 & .5111 & .01809 & 92 & 62 \\
$21906 \ldots \ldots \ldots$ & 31.4 & 10.4800 & .8062 & .02563 & 88 & 27 \\
$21908 \ldots \ldots \ldots$ & 28.8 & 3.5574 & .5929 & .02056 & 92 & 9 \\
$21909 \ldots \ldots \ldots$ & 30.9 & 12.1819 & .7166 & .02317 & 86 & 29 \\
$21911 \ldots \ldots \ldots$. & 29.5 & 8.4593 & .6597 & .02209 & 90 & 23 \\
\hline
\end{tabular}


TABLE 35.-Relation of size of head to yield, height, and tillering of plant-Continued.

SIZE OF HEAD, 28 TO 32 KERNELS-Continued.

\begin{tabular}{|c|c|c|c|c|c|c|}
\hline $\begin{array}{c}\text { Record num- } \\
\text { ber. }\end{array}$ & $\begin{array}{l}\text { Size of } \\
\text { head. }\end{array}$ & $\begin{array}{l}\text { Yield per } \\
\text { plant } \\
\text { (grams). }\end{array}$ & $\begin{array}{l}\text { Yield per } \\
\text { head } \\
\text { (grams). }\end{array}$ & $\begin{array}{l}\text { Weight of } \\
\text { average } \\
\text { kernel } \\
\text { (grams). }\end{array}$ & $\begin{array}{l}\text { Height } \\
\text { (cm.). }\end{array}$ & Tillering. \\
\hline $22206 \ldots \ldots \ldots$ & 29.2 & 2.5712 & 0.5142 & 0.01720 & 70 & 9 \\
\hline $22211 \ldots \ldots \ldots$ & 28.0 & 11.5675 & .5784 & .02062 & 88 & 59 \\
\hline $26107 \ldots \ldots \ldots$ & 28.8 & 2.0390 & .4078 & .01416 & 67 & 6 \\
\hline $27005 \ldots \ldots \ldots$ & 28.9 & 16.4120 & .5471 & .01895 & 77 & 40 \\
\hline $27206 \ldots \ldots \ldots$ & 28.8 & 19.1854 & .7106 & .02469 & 90 & 49 \\
\hline $27306 \ldots \ldots$. & 28.5 & 13.3011 & .5542 & .01945 & 88 & 48 \\
\hline $27308 \ldots \ldots$. & 31.7 & 4.5123 & .5640 & .01777 & 88 & 11 \\
\hline $27509 \ldots \ldots$ & 30.4 & 5. 3615 & .6702 & .02206 & 73 & 9 \\
\hline $32206 \ldots \ldots \ldots$ & 28.2 & 10.4036 & .5779 & .02052 & 71 & 26 \\
\hline $32605 \ldots \ldots \ldots$ & 28.1 & 5.2268 & .6533 & .02323 & 71 & 9 \\
\hline $32606 \ldots \ldots \ldots$ & 31.3 & 2.0162 & .6721 & .02145 & 69 & 3 \\
\hline $34205 \ldots \ldots \ldots$ & 30.9 & 9.1498 & .6100 & .01972 & 78 & 19 \\
\hline $34208 \ldots \ldots \ldots$ & 31.2 & 2.9886 & .5977 & .01916 & 66 & 5 \\
\hline $37305 \ldots \ldots .$. & 30.9 & 6. 1394 & .6139 & .01987 & 58 & 12 \\
\hline $38505 \ldots \ldots \ldots$ & 29.6 & 12. 1088 & .6373 & .02252 & 70 & 21 \\
\hline $38506 \ldots \ldots \ldots$ & 28.3 & 1.6799 & .5600 & .01975 & 54 & 3 \\
\hline $38605 \ldots \ldots \ldots$ & 30.5 & 1. 2124 & .6062 & .01987 & 55 & 2 \\
\hline $39405 \ldots \ldots \ldots$ & 31.9 & 9.3541 & .6681 & .02093 & 74 & 18 \\
\hline $39606 \ldots \ldots \ldots$ & 31.4 & 4. 6383 & .4217 & .01341 & 64 & 18 \\
\hline $40305 \ldots \ldots \ldots$ & 29.8 & 3.6003 & .6000 & .02011 & 62 & 6 \\
\hline $44505 \ldots \ldots \ldots$ & 30.9 & 5.9990 & .5453 & .01764 & 69 & 25 \\
\hline $45005 \ldots . .$. & 29.4 & 3.2340 & .4042 & .01376 & 66 & 9 \\
\hline $45805 \ldots \ldots \ldots$ & 31.0 & 1.5298 & .3824 & .01234 & 48 & 4 \\
\hline $46107 \ldots \ldots \ldots$ & 31.9 & 8.3935 & .5595 & .01756 & 79 & 27 \\
\hline $50905 \ldots \ldots$. & 31.6 & 2. 3982 & .3426 & .01085 & 68 & 10 \\
\hline $50906 \ldots \ldots$. & 28.5 & 1. 7280 & .4320 & .01516 & 58 & 5 \\
\hline $55005 \ldots \ldots \ldots$ & 30.2 & 7.9684 & .6129 & .02028 & 75 & 19 \\
\hline $55006 \ldots \ldots$. & 30.1 & 7.1852 & .4790 & .01593 & 80 & 19 \\
\hline $55007 \ldots \ldots \ldots$ & 29.5 & 2.1571 & .5393 & .01828 & 65 & 7 \\
\hline $55206 \ldots \ldots \ldots$ & 30.4 & 11.3592 & .5978 & .01965 & 82 & 27 \\
\hline $55306 \ldots \ldots \ldots$ & 30.6 & 4. 1323 & .5903 & .01931 & 77 & 17 \\
\hline $55307 \ldots \ldots \ldots$ & 31.1 & 5. 6864 & .5169 & .01663 & 80 & 19 \\
\hline $55507 \ldots \ldots \ldots$ & 31.5 & 9.8228 & .6139 & .01949 & 95 & 28 \\
\hline $56106 \ldots \ldots \ldots$ & 28.0 & 12.0161 & .5224 & .01866 & 90 & 33 \\
\hline $57006 \ldots \ldots$ & 30.5 & - 10.1836 & .4427 & .01453 & 88 & 41 \\
\hline $57407 \ldots \ldots \ldots$ & 31.8 & 14.9992 & .6250 & .01968 & 92 & 41 \\
\hline $58207 \ldots \ldots \ldots$ & 30.7 & 4. 2207 & .4221 & .01375 & 75 & 18 \\
\hline $58505 \ldots \ldots \ldots$ & 31.1 & 7.4516 & .6210 & .02730 & 80 & 18 \\
\hline $58806 \ldots \ldots \ldots$ & 31.7 & 1.9469 & .6489 & .02049 & 65 & 7 \\
\hline $59606 \ldots \ldots$ & 29.8 & 9.7084 & .5109 & .01712 & 80 & 37 \\
\hline $63505 \ldots \ldots$ & 29.7 & 4.0230 & $: 5747$ & .01934 & 66 & 8 \\
\hline $65307 \ldots \ldots$. & 31.1 & 7.0051 & .5838 & .01878 & 74 & 17 \\
\hline $66005 \ldots \ldots \ldots$ & 30.8 & 7.6690 & .6391 & .02073 & 75 & 22 \\
\hline $69506 \ldots \ldots$ & 30.1 & 13.5696 & .6168 & .02047 & 73 & 24 \\
\hline $71905 \ldots \ldots$ & 29.3 & 28.2136 & .6561 & .02239 & 80 & 46 \\
\hline $72406 \ldots \ldots$ & 30.7 & 8.2929 & .5923 & .01929 & 70 & 15 \\
\hline $72706 \ldots \ldots$ & 29.5 & 14. 6802 & .7340 & .02481 & 80 & 27 \\
\hline $72707 \ldots \ldots$ & 28.1 & 4. 5806 & .5726 & .02036 & 72 & 8 \\
\hline $76206.2 \ldots \ldots$ & 29.8 & 5.4411 & .3627 & .01217 & $7 \overline{3}$ & 30 \\
\hline $88906 \ldots . . .$. & 30.3 & 9.9034 & .5502 & .01814 & 80 & 21 \\
\hline $92408 \ldots \ldots \ldots$ & 29.6 & 3. 7820 & .5403 & .01827 & 81 & 7 \\
\hline $92908 \ldots . . . .$. & 31.2 & 3.2388 & .5398 & .01732 & 76 & 7 \\
\hline $94205 \ldots . .$. & 31.3 & 1.2117 & .4039 & .01893 & 55 & 6 \\
\hline $94207 \ldots \ldots$. & 29.9 & 13.7057 & .5711 & .01909 & 83 & 31 \\
\hline $94209 \ldots \ldots \ldots$ & 31.7 & 3. 6006 & .6001 & .01895 & 75 & 7 \\
\hline $94406 \ldots \ldots$. & 28.9 & 10.5556 & .5556 & .01923 & 82 & 22 \\
\hline $94605 \ldots \ldots \ldots$ & 28.0 & .7319 & .3659 & .01307 & 68 & 7 \\
\hline $94606 \ldots \ldots \ldots$ & 29.9 & 11.8435 & .5383 & .07544 & 84 & 23 \\
\hline $94905 \ldots \ldots \ldots$ & 31.8 & 4. 4423 & .4936 & .01553 & 75 & 11 \\
\hline $94906 \ldots \ldots \ldots$ & 29.8 & 12.3862 & .5385 & .01808 & 91 & 24 \\
\hline $95706 \ldots \ldots \ldots$ & 29.7 & 5.1629 & .5736 & .01934 & 82 & 9 \\
\hline Average. & 30.1 & 7.4992 & .5598 & .01958 & 74.5 & 19.4 \\
\hline
\end{tabular}


RELATION OF SIZE OF HEAD TO YIELD, ETC.

TABLE 35.-Relation of size of head to yield, height, and tillering of plant-Continued.

SIZE OF HEAD, 32 TO 36 KERNELS.

\begin{tabular}{|c|c|c|c|c|c|c|}
\hline $\begin{array}{c}\text { Record num- } \\
\text { ber. }\end{array}$ & $\begin{array}{l}\text { Size of } \\
\text { head. }\end{array}$ & $\begin{array}{l}\text { Yield per } \\
\text { plant } \\
\text { (grams). }\end{array}$ & $\begin{array}{l}\text { Yield per } \\
\text { head } \\
\text { (grams). }\end{array}$ & $\begin{array}{l}\text { Weight of } \\
\text { average } \\
\text { kernel } \\
\text { (grams). }\end{array}$ & $\begin{array}{l}\text { Height } \\
\text { (em.). }\end{array}$ & Tillering. \\
\hline $17307 \ldots \ldots$ & 34.5 & 3.1454 & 0.7863 & 0.02279 & 70 & 8 \\
\hline $18905 \ldots . . .$. & 34.3 & 1.4864 & .4955 & .01443 & 50 & 4 \\
\hline $26105 \ldots \ldots \ldots$ & 32.7 & 1.8242 & $.45 t: 0$ & .01393 & 69 & 13 \\
\hline $269617 \ldots \ldots$ & 34.0 & 1.8276 & .6092 & .01792 & 55 & 8 \\
\hline $28 \$ 06 \ldots . . .$. & 34.2 & 14.4630 & .7232 & .02111 & 75 & 30 \\
\hline $34405 \ldots \ldots \ldots$ & 34.5 & 4. 1281 & .6881 & .01994 & 62 & 8 \\
\hline $34606 \ldots \ldots$. & 35.0 & 6. 1962 & .7745 & .02213 & 61 & 13 \\
\hline $36905 \ldots . . .$. & 33.4 & 5.0200 & .6275 & .01880 & 58 & 7 \\
\hline $39205 \ldots \ldots \ldots$ & 32.2 & 21.5399 & .6731 & .02089 & 82 & 40 \\
\hline $42405 \ldots . .$. & 33.0 & 1.4892 & .7446 & .02251 & 60 & 2 \\
\hline $42905 \ldots . . .$. & 33.5 & 1.2499 & .6249 & $.018 t 66$ & 68 & 4 \\
\hline $48506 \ldots . .$. & 32.7 & 9.4585 & $.556,4$ & .01701 & 82 & 30 \\
\hline $49505 \ldots \ldots \ldots$ & 33.5 & 1.2716 & .6358 & .01898 & 60 & 3 \\
\hline $51005 \ldots . .$. & 34.5 & 15.5835 & .6233 & -.01804 & 75 & 32 \\
\hline $55008 \ldots \ldots \ldots$ & 33.7 & 17.4226 & .6222 & .01846 & 82 & 30 \\
\hline $55305 \ldots \ldots \ldots$ & 33.4 & 2.5160 & .5032 & .01507 & 75 & 12 \\
\hline $55308 \ldots \ldots$. & 33.1 & 9.5078 & .7923 & .02395 & 79 & 28 \\
\hline $55605 \ldots \ldots$ & 33.3 & 10.9180 & .7279 & .02184 & 89 & 23 \\
\hline $55607 \ldots \ldots$ & 34.5 & 2. 3931 & .5983 & .01734 & 77 & 7 \\
\hline $55608 \ldots . .$. & 33.5 & 22.5848 & .9034 & .02699 & 95 & 31 \\
\hline $57007 \ldots \ldots$ & 33.6 & 3. 3176 & .6635 & .01975 & 90 & 9 \\
\hline $57406 \ldots \ldots$ & 33.7 & 2. 4923 & .6231 & .01846 & 92 & 14 \\
\hline $57408 \ldots \ldots$ & 35.0 & 12.2004 & .7177 & .02047 & 90 & 26 \\
\hline $58805 \ldots \ldots$ & 35.1 & 23.1471 & .7014 & .01999 & 78 & 51 \\
\hline $60605 \ldots \ldots$ & 35.0 & .5952 & .5952 & .01701 & 57 & 4 \\
\hline $69305 \ldots \ldots$. & 34.3 & 2.0430 & .6810 & .01984 & 70 & 7 \\
\hline $72405 \ldots \ldots$ & 35.5 & 8.4415 & 1.4069 & .03963 & 67 & 6 \\
\hline $72708 \ldots \ldots$ & 33.2 & 9.0386 & .7532 & .02270 & 78 & 12 \\
\hline $73308 \ldots \ldots$. & $34 . \overline{7}$ & 14.2986 & .7944 & .02291 & 74 & 23 \\
\hline $85206 \ldots \ldots$ & 34.2 & 4.9315 & .4483 & .01312 & 69 & 13 \\
\hline $88605 \ldots . .$. & 34.5 & 1.6362 & .8181 & .02731 & 70 & 3 \\
\hline $91305 \ldots \ldots \ldots$ & 34.5 & 3.0940 & .7735 & .02242 & 76 & 6 \\
\hline $92208 \ldots . . .$. & 35.3 & 6.6206 & .6621 & .01876 & 78 & 17 \\
\hline $92406 \ldots \ldots \ldots$ & 34.5 & 8.2366 & .7488 & .02168 & 81 & 17 \\
\hline $92409 \ldots \ldots$ & 35.0 & 5.7131 & .6348 & .01814 & 81 & 13 \\
\hline $92905 \ldots \ldots \ldots$ & 35.2 & 2.7000 & .5400 & .01534 & 75 & 6 \\
\hline $92909 \ldots$ & 33.1 & 10. 1363 & .6335 & .01916 & 86 & 21 \\
\hline $95509 \ldots \ldots$. & 34.5 & 2.9475 & .7369 & .02136 & 74 & 4 \\
\hline Average. & 34.1 & 7.2530 & .6868 & .02023 & 73.9 & 15.4 \\
\hline
\end{tabular}

SIZE OF HEAD, 36 KERNELS AND OVER.

\begin{tabular}{|c|c|c|c|c|c|c|}
\hline $18906 \ldots$ & 65.0 & 0.9229 & 0.9229 & 0.01420 & 67 & 5 \\
\hline $21813 \ldots \ldots$ & 43.2 & 4.0258 & .8051 & .01877 & 80 & 21 \\
\hline $34206 \ldots \ldots \ldots$ & 40.5 & 1.5940 & .7970 & .01968 & 74 & 5 \\
\hline $37707 \ldots \ldots$. & 38.6 & 3.3004 & .6601 & .01710 & 64 & 5 \\
\hline $40205 \ldots \ldots \ldots$ & 38.8 & 3. 6302 & .7260 & .01871 & 65 & 11 \\
\hline $40505 \ldots . .$. & 42.5 & 4. 1546 & 1.0386 & .02444 & 60 & 4 \\
\hline $43405 \ldots . .$. & 41.3 & 2.8000 & .9333 & .02258 & 64 & 3 \\
\hline $46105 \ldots \ldots$. & 37.1 & 4. 6146 & .6592 & .01775 & 73 & 8 \\
\hline $48705 \ldots \ldots$. & 44.0 & 4.3615 & .7269 & .01652 & 80 & 7 \\
\hline $48706 \ldots . .$. & 47.4 & 6. 1986 & .7748 & .01635 & 78 & 12 \\
\hline $55508 \ldots \ldots \ldots$ & 36.0 & 3.7407 & .6222 & .01732 & 73 & 12 \\
\hline $57405 \ldots \ldots .$. & 41.0 & .8328 & .8328 & .02031 & 73 & 1 \\
\hline $57805 \ldots \ldots$. & 38.6 & 4.8988 & .6998 & .01814 & 76 & 17 \\
\hline $57905 \ldots \ldots$ & 36.8 & 2.4731 & .4122 & .01118 & 74 & 17 \\
\hline $58705 \ldots \ldots$ & 58.7 & 2.5436 & .6359 & .01082 & 68 & 11 \\
\hline $58905 \ldots \ldots$ & 42.5 & 2. 3031 & .5758 & .01355 & 66 & 13 \\
\hline $59605 \ldots \ldots$ & 38.2 & 7. 1828 & .7183 & .01880 & 77 & 30 \\
\hline $62805 \ldots \ldots$ & 37.0 & 1.3451 & .4484 & .01212 & 70 & 14 \\
\hline $6 f 006 \ldots . .$. & 52.3 & 6.0090 & .8584 & .01642 & 73 & 12 \\
\hline $72806 \ldots \ldots$ & 36.7 & 2.0970 & .6990 & .01906 & 62 & 5 \\
\hline $73306 \ldots \ldots$ & 37.6 & 8.5373 & .7761 & .02062 & 78 & 20 \\
\hline $81505 \ldots \ldots \ldots$ & 48.7 & $2.832 \pi$ & .9442 & .01940 & 78 & 7 \\
\hline $84905 \ldots \ldots$ & 37.0 & .7130 & .7130 & .01927 & 47 & 4 \\
\hline $92 \times 16 \ldots . .$. & 36.2 & 2.8816 & .5763 & .01592 & 75 & 7 \\
\hline $95505 \ldots \ldots$ & 37.0 & .3146 & .3146 & .00850 & 79 & 3 \\
\hline Average. & . 42.1 & 3.3723 & .7148 & .01710 & 71.0 & 10.2 \\
\hline
\end{tabular}


TABLE 35.-Summary of relation of size of head to yield, height, and tillering of plant.

\begin{tabular}{|c|c|c|c|c|c|c|c|}
\hline $\begin{array}{l}\text { Classification according to } \\
\text { number of kernels on } \\
\text { head. }\end{array}$ & $\begin{array}{l}\text { Number } \\
\text { of plants. }\end{array}$ & $\begin{array}{l}\text { Average } \\
\text { number } \\
\text { of kernels } \\
\text { on spike. }\end{array}$ & $\begin{array}{l}\text { Yield per } \\
\text { plant } \\
\text { (grams). }\end{array}$ & $\begin{array}{l}\text { Yield per } \\
\text { head } \\
\text { (gram). }\end{array}$ & $\begin{array}{l}\text { Weight of } \\
\text { average } \\
\text { kernel } \\
\text { (gram) }\end{array}$ & $\begin{array}{l}\text { Height } \\
(\mathrm{cm} .) \text {. }\end{array}$ & Tillering. \\
\hline $\begin{array}{l}\text { Below } 16 \ldots \ldots \\
16 \text { to } 20 \ldots \ldots \\
20 \text { to } 24 \ldots \ldots \\
24 \text { to } 28 \ldots \ldots \\
28 \text { to } 32 \ldots \ldots \ldots \\
32 \text { to } 36 \ldots \ldots \\
\text { More } \operatorname{man} 36 \ldots \ldots\end{array}$ & $\begin{array}{l}18 \\
36 \\
80 \\
84 \\
73 \\
38 \\
25\end{array}$ & \begin{tabular}{l|}
13.3 \\
18.4 \\
22.2 \\
25.9 \\
30.1 \\
34.1 \\
42.1
\end{tabular} & $\begin{array}{l}1.3169 \\
3.7758 \\
6.8466 \\
7.5207 \\
7.4992 \\
7.2530 \\
3.3723\end{array}$ & $\begin{array}{r}0.2654 \\
.3383 \\
.4355 \\
.4848 \\
.5598 \\
.6868 \\
.7148\end{array}$ & $\begin{array}{r}0.02059 \\
.01862 \\
.01953 \\
.01874 \\
.01958 \\
.02023 \\
.01710\end{array}$ & $\begin{array}{l}55.2 \\
64.1 \\
73.8 \\
73.8 \\
74.5 \\
73.9 \\
71.0\end{array}$ & $\begin{array}{r}6.9 \\
13.7 \\
21.4 \\
21.2 \\
19.4 \\
15.4 \\
10.2\end{array}$ \\
\hline
\end{tabular}

TABLE 37.-Relation of yield of plant to height and tillering, and to the yield per head.

\begin{tabular}{|c|c|c|c|c|c|}
\hline $\begin{array}{c}\text { Classification according to yield per plant, in } \\
\text { grams. }\end{array}$ & $\begin{array}{l}\text { Number } \\
\text { of plants. }\end{array}$ & $\begin{array}{c}\text { Yield per } \\
\text { plant } \\
\text { (grams). }\end{array}$ & $\begin{array}{l}\text { Height } \\
\text { (cm.). }\end{array}$ & Tillering. & $\begin{array}{l}\text { Yield per } \\
\text { head } \\
\text { (gram). }\end{array}$ \\
\hline 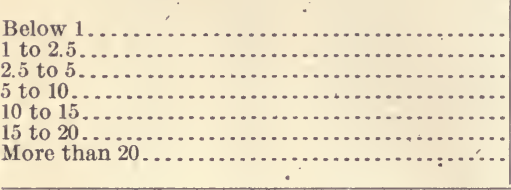 & $\begin{array}{r}31 \\
67 \\
87 \\
93 \\
51 \\
20 \\
5\end{array}$ & $\begin{array}{r}0.6050 \\
1.7673 \\
3.5526 \\
7.6485 \\
12.2862 \\
17.1908 \\
23.2829\end{array}$ & $\begin{array}{l}56.5 \\
62.2 \\
69.1 \\
75.4 \\
84.4 \\
84.6 \\
85.2\end{array}$ & \begin{tabular}{r|}
3.7 \\
7.0 \\
11.6 \\
22.1 \\
32.3 \\
42.9 \\
43.2
\end{tabular} & $\begin{array}{r}0.3553 \\
.4740 \\
.4917 \\
.5320 \\
.5592 \\
.5310 \\
.6865\end{array}$ \\
\hline
\end{tabular}

TABLE 38---Relation of yield per head to yield, height, and tillering of plant, and to weight of average kernel.

\begin{tabular}{|c|c|c|c|c|c|c|}
\hline $\begin{array}{l}\text { Classification according to yield } \\
\text { per head, in grams. }\end{array}$ & $\begin{array}{l}\text { Number } \\
\text { of plants: }\end{array}$ & $\begin{array}{l}\text { Yield per } \\
\text { head } \\
\text { (gram). }\end{array}$ & $\begin{array}{l}\text { Yield per } \\
\text { plant } \\
\text { (grams). }\end{array}$ & $\begin{array}{l}\text { Height } \\
\text { (cm.). }\end{array}$ & Tillering. & $\begin{array}{l}\text { Weight of } \\
\text { average } \\
\text { kernel } \\
\text { (gram). }\end{array}$ \\
\hline $\begin{array}{l}\text { Below } 0.300 \ldots \ldots \\
0.300 \text { to } 0.400 \ldots \ldots \\
0.400 \text { to } 0.500 \ldots \ldots \\
0.500 \text { to } 0.600 \ldots \ldots \\
0.600 \text { to } 0.700 \ldots \ldots \\
0.700 \text { to } 0.800 \ldots \ldots \\
\text { More than } 0.800 \ldots \ldots\end{array}$ & $\begin{array}{l}30 \\
62 \\
98 \\
78 \\
50 \\
25 \\
12\end{array}$ & $\begin{array}{r}0.2484 \\
.3567 \\
.4524 \\
.5477 \\
.6372 \\
.7456 \\
.9229\end{array}$ & $\begin{array}{l}1.6939 \\
3.7365 \\
6.7326 \\
9.5646 \\
7.6214 \\
4.4523 \\
5.7687\end{array}$ & $\begin{array}{l}60.8 \\
65.6 \\
72.8 \\
76.6 \\
74.3 \\
75.2 \\
73.7\end{array}$ & $\begin{array}{r}11.4 \\
15.5 \\
19.9 \\
21.8 \\
17.3 \\
18.6 \\
10.3\end{array}$ & $\begin{array}{r}0.01586 \\
.01737 \\
.01847 \\
.02073 \\
.02056 \\
.02179 \\
.02151\end{array}$ \\
\hline
\end{tabular}

\section{SUMMARY AND CONCLUSIONS.}

As between wheat kernels of the same variety raised under similar conditions, those kernels having a high percentage of proteid material have a lower specific gravity, weigh slightly less, and occupy a smaller volume than kernels having a smaller percentage of proteids.

As between individual spikes and individual plants, the same relations obtain.

As between individual plants in different years, these relations do not hold.

The quality of high proteid content and its correlated properties may be due to immaturity in the kernel, or they may belong to the normal and fully ripened kernel.

As between kernels, spikes, and plants, those kernels of greater weight contain a larger weight of proteids - this in spite of the fact that they contain a lower percentage. 
Plants bearing the largest number of kernels have kernels of more than medium but not the greatest weight, as do also plants producing the greatest weight of kernels. The same is true of plants producing the greatest weight of proteid matter and gluten.

Heavy seed wheat drilled at the rate of $1 \frac{1}{2}$ bushels per acre produced a much larger crop of seed the first year of the separation than did light seed drilled at the same rate, but by continuing the separation of the respective crops and selecting heavy seed from the crop grown from heavy seed, and light seed from the crop grown from light seed, the difference in yield in three or four years was small.

After the first year of separation the light seed produced a greater amount of proteids per acre than did the heavy seed.

A determination of the total or of the proteid nitrogen content in the kernels on one row of spikelets of wheat affords a fairly close estimate of the same constituents in the kernels on the other row of spikelets.

A determination of the total or of the proteid nitrogen content in the kernels on one-half of the spikes on a wheat plant will give a very good estimate of the same constituents in the kernels on the other spikes, provided there are at least an average number of spikes on the plant.

There may be quite a large variation in the proteid nitrogen content of different spikes on the same wheat plant.

Determinations of the proteid nitrogen content of 800 spikes of wheat of the same variety representing different plants showed a variation of from 1.12 to 4.95 per cent of proteid nitrogen, and 351 plants of the same variety the following year varied from 1.20 to 5.85 per cent.

The proportion of gluten to proteids in kernels of different wheat plants may vary considerably. A determination of proteid nitrogen is therefore not always a guide to the gluten content of the wheat. Selection for improvement should be based on the determination of gluten.

Wheat plants having kernels high in gluten contain a smaller proportion of other proteids than do plants of medium or low gluten content.

In wheat of the same variety, raised in the same field in the same year, the ratio of gliadin to glutenin was practically the same in plants of low, medium, and high proteid nitrogen content.

It may therefore be assumed that an increase in the gluten content of a given variety of wheat raised in the same region would carry with it a corresponding improvement in its value for bread making, although there might be fluctuations from year to year in the quality of the gluten. 
The content of proteid nitrogen, the kernel weight, and the total proteid nitrogen production by the wheat plant are hereditary qualities.

There is a tendency for plants possessing any of these qualities in an extreme degree to produce progeny in which the same qualities approach more closely to the average, but certain exceptional plants may transmit the same or more extreme qualities.

The yield of grain per plant after a severe winter was decreased in proportion to the susceptibility of the plant to cold. The effect of the cold caused the plant to produce a less number of heads, or, in other words, to tiller less.

The early-maturing plants yielded the most grain, and those ripening later produced in each case less when grouped into ripening periods of four days, extending through more than three weeks' time.

The early-maturing plants produced grain of slightly lower nitrogen content than the later maturing plants, and the number of grams of proteid nitrogen in the average kernel was likewise less in the early-maturing plants.

Plants with heads of slightly more than medium size produced the largest yields of grain, and were taller than plants with either larger or smaller heads. Plants with heads of medium size, or slightly less, tillered most extensively.

The weight of the average kernel did not increase with the size of the head, nor did it decrease, except on the very largest heads.

The largest yielding plants were the tallest and tillered most.

\section{U}
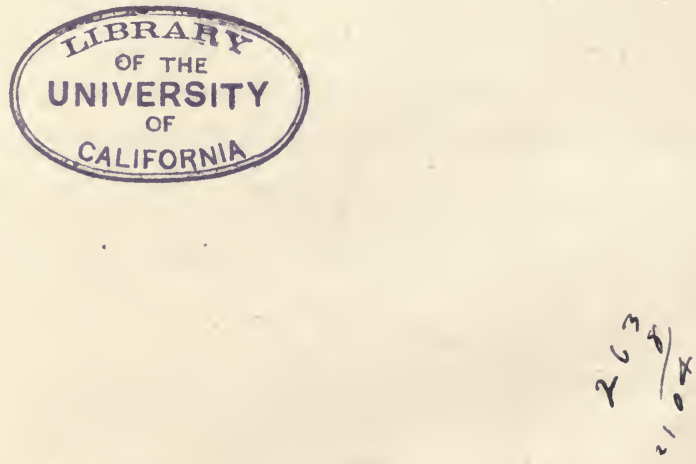



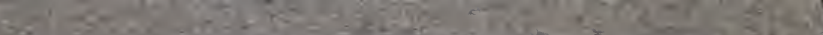

UNIVERSITY OF CALIFORNIA LIBRARY

THIS BOOY IS DUE ON THE LAST DATE STAMPED BELOW

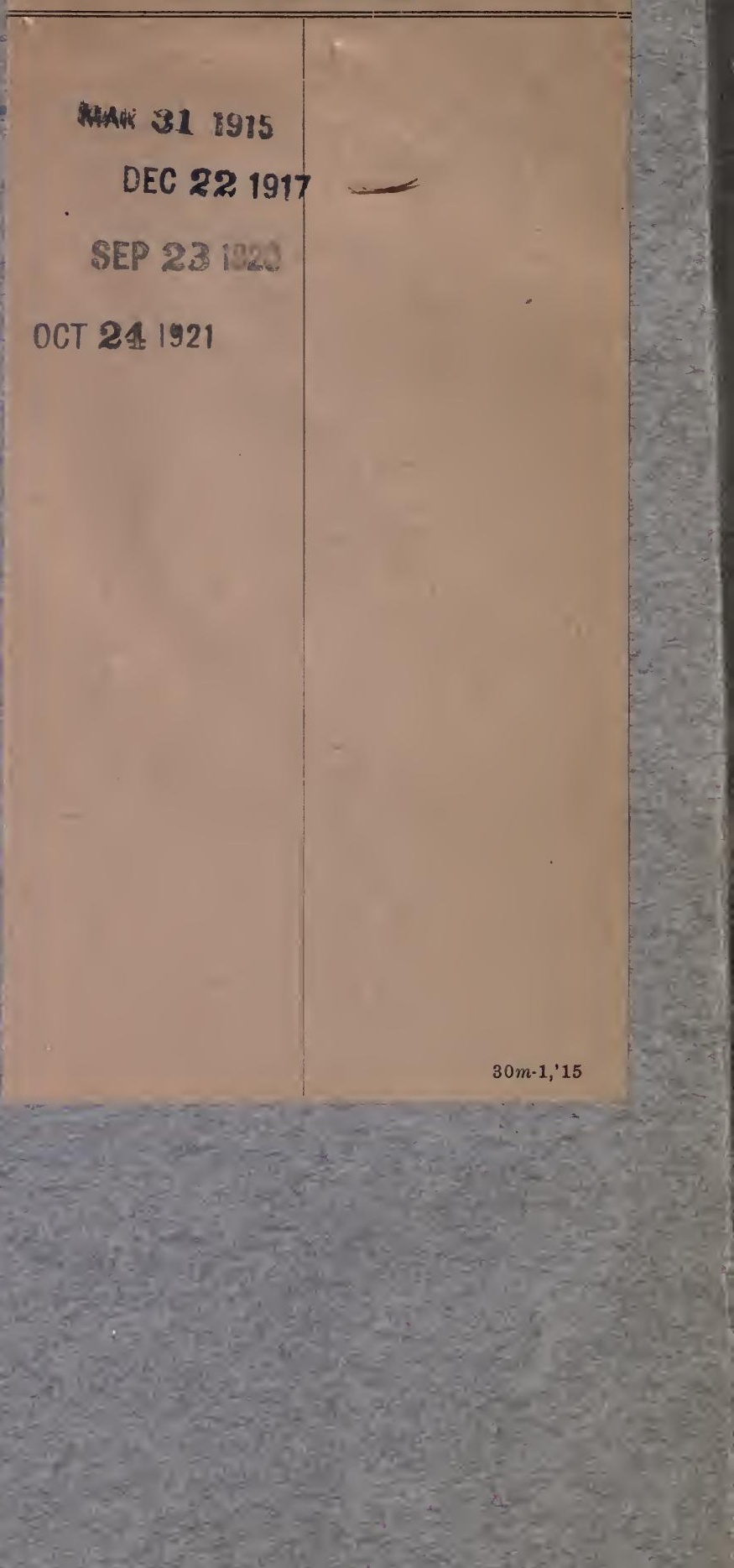


